\title{
APPLICATIONS OF ANCESTRY ESTIMATION IN CATTLE POPULATION GENETICS
}

\begin{abstract}
A Dissertation Presented to the Faculty of the Graduate School at the University of Missouri-Columbia
\end{abstract}

\begin{abstract}
In Partial Fulfillment of the Requirements for the Degree Doctor of Philosophy
\end{abstract}

\author{
by \\ TAMAR CRUM \\ Dr. Jeremy Taylor, Dissertation Advisor
}

May 2019 


\title{
APPROVAL PAGE
}

The undersigned, appointed by the Dean of the Graduate School, have examined the dissertation entitled:

\section{APPLICATIONS OF ANCESTRY ESTIMATION IN CATTLE POPULATION GENETICS}

\begin{abstract}
Presented by Tamar Crum, a candidate for the degree of Doctor of Philosophy, and hereby certify that in their opinion it is worthy of acceptance.
\end{abstract}

Dr. Jeremy F. Taylor, Animal Sciences, UMC

Dr. Robert D. Schnabel, Animal Sciences, UMC

Dr. Jared E. Decker, Animal Sciences, UMC

Dr. J. Chris Pires, Biological Sciences, UMC 


\section{Dedication}

This dissertation is dedicated to my family, who have provided unwavering support and encouragement throughout the completion of this degree. To my parents, Jim and Jan Adcock, thank you for instilling in me the values of hard work and responsibility and also for always keeping me grounded, pushing me to chase my dreams, and keeping faith at the forefront. I will never be able to express enough gratitude for being afforded the opportunity to travel the country showing cattle. The network of people, leadership skills, responsibility, and work ethic developed because of time spent in the show barn and on the show circuit is second to none. To my siblings, Taryn, Dean and Del, thank you for simply being YOU. Each of you bring something special to my life - your encouragement, comedic relief, and support has been greatly appreciated. To Wayne and Kay, thank you for always being willing to pick up my chores and surrender your kitchen table when deadlines had to be met, we couldn't have managed to keep Crum Cattle moving forward without you. To Papaw, well, there really aren't words. I wrote an essay in the $1^{\text {st }}$ grade about my hero, Papaw Howard. My writing has vastly improved since then, but my hero remains unchanged. Thank you for living an example of an unwavering love and faithfilled life. Last but certainly not least, thank you to my husband, Cody. I know at the end of this, my name will be on the diploma, but to be honest, it probably should be addressed to both of us. Thank you for loving me through it, even though I am certain there were times it was not easy. I am so excited to see what our next adventures in life will bring! 
It's simple really. Without the support, encouragement, guidance, faith, and love that each of you have provided, I would not be the person I am today - and for that I will be forever grateful. This is for you.

\section{My sincerest thanks,}

Tamar 


\section{ACKNOWLEDGEMENTS}

There are many individuals that have played vital roles in my educational, leadership, and personal development over the past several years. While some had only fleeting interactions, those interactions still helped shape me into the person I am today. It would be impossible to thank everyone who has helped me reach this point, but I would like to express my appreciation to several individuals who have gone above and beyond. Foremost, I would like to thank my advisor, Dr. Jerry Taylor for his guidance, patience, and desire to help me succeed. I appreciate his willingness to help me design a research plan, educational curriculum, and path that suited my interests and desires - with your support and patience I was eventually able to envision a future career path that I had not previously considered. I am beyond humbled to have been afforded the opportunity to be one of the last "Taylor students."

The computational and data management aspects of my projects have allowed me the privilege of having a second mentor, Dr. Bob Schnabel. I have learned an incredible amount about the construction of repeatable processes and thinking beyond the "here and now." It is because of him, I gained an appreciation for data management, handling, storage, and processing. I appreciate his ability to offer advice on the fly, keep me grounded, and for his constant support and his ability push me to think outside the box. l'd also be remiss to not extend a heartfelt thank you to Dr. Jared Decker, Dr. J. Chris Pires, Dr. JaeWoo Kim, Helen Yampara-lquise, and members of the MU Genomics 
group for facilitating a sense of community within the lab that has encouraged me to reach my full potential.

Finally, thank you to the community of people and businesses that opened up their homes, offices, and Wi-Fi passwords affording me a space to set up "camp" and work from Illinois. A special thank you to Wenger Ag Solutions, Heartland Ag. Group Ltd, and Dalton and Julie Anne Shepherd... your Wi-Fi, but mainly your friendships are greatly appreciated! 


\section{TABLE OF CONTENTS}

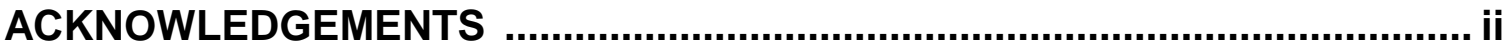

LIST OF FIGURES ................................................................................. viii

LIST OF TABLES

LIST OF SUPPLEMENTARY FIGURES …..................................................... xiii

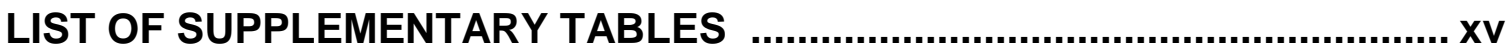

CHAPTER 1

APPLICATIONS OF ANCESTRY ESTIMATION IN CATTLE ............................ 1

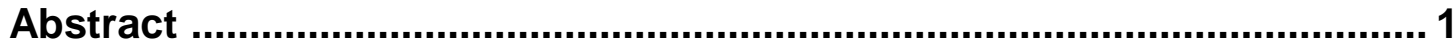

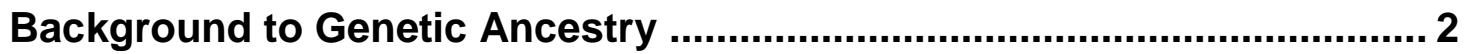

Ancestry Prediction in Humans ................................................................. 4

Domestication of Cattle .......................................................................... 14

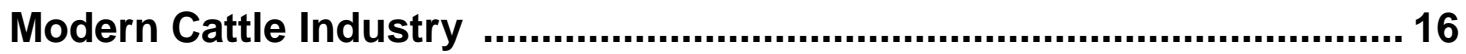

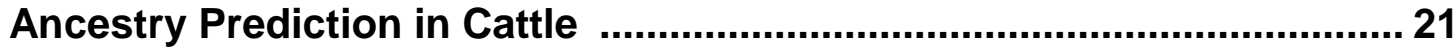

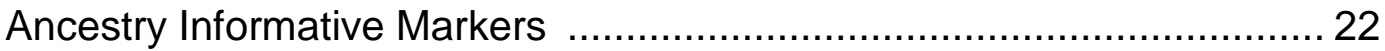

Global Ancestry of Admixed Populations ............................................. 23

Local Ancestry of Admixed Populations ................................................. 25

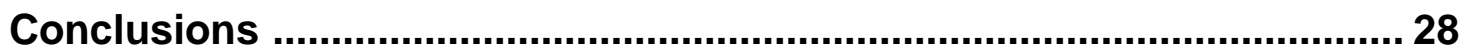

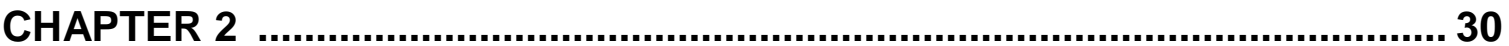

CRUMBLER: A Tool for the Prediction of Ancestry in Cattle ......................30

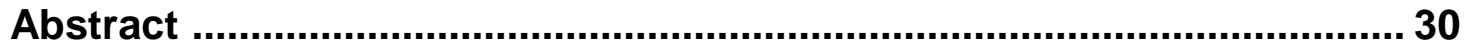

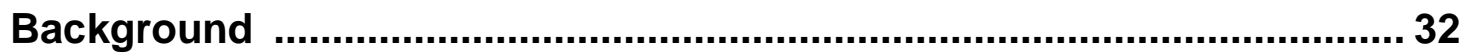

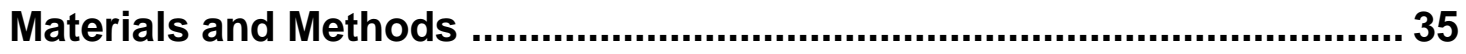




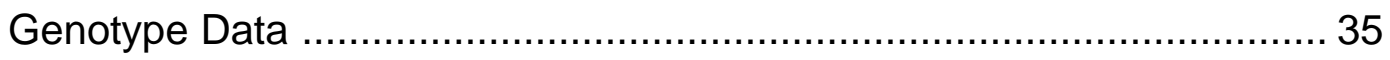

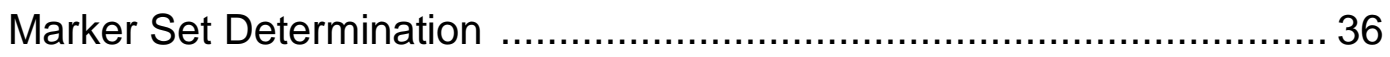

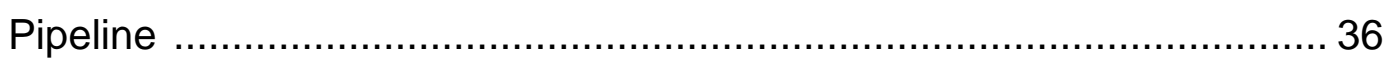

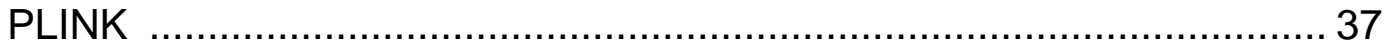

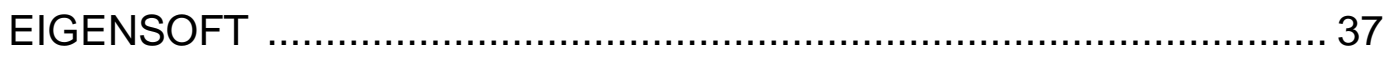

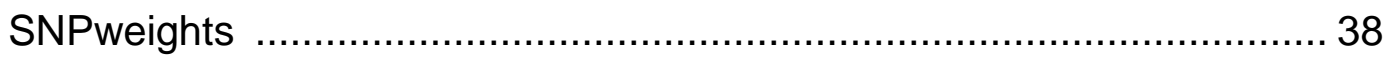

Reference Panel Development ….................................................. 39

FastSTRUCTURE Analysis to Identify Candidate Reference Panel Individuals

SNPweights Analyses to Refine and Validate Reference Panel Members

Breeds with Open Herdbooks ......................................................... 41

Additional Reference Panel Filtering Using SNPweights ...................... 42

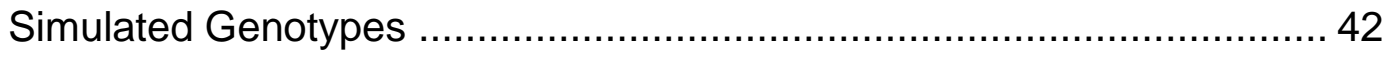

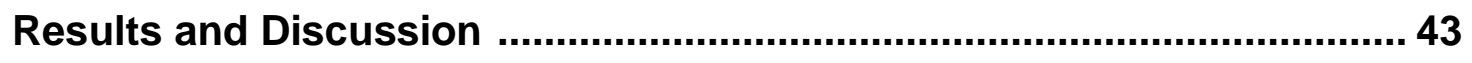

Reference Panel Development ..................................................... 44

Reference population sample size ……………….............. 46

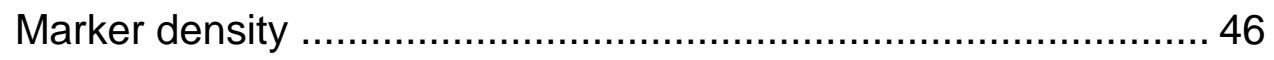

Assignment thresholds ........................................................ 47

Reference panel definition .................................................. 48

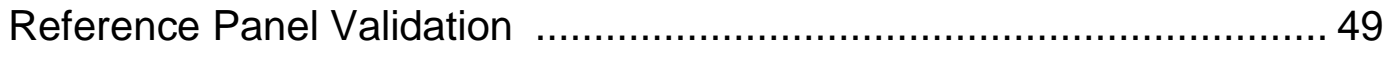

Registered fullblood animals ............................................... 50

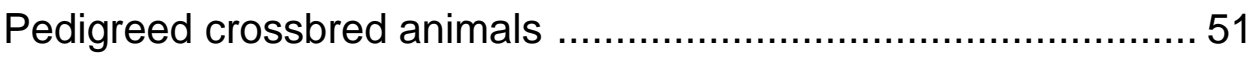

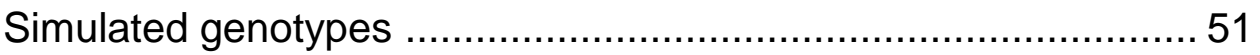


Admixture

Broader Application Using Additional Commercially Available Assays ... 56

Conclusions

Figures

Tables

CHAPTER 3 86

HAPLOTYPIC MOSAICS OF ADVANCED GENERATION COMPOSITE BREED CATTLE FORMED AS CROSSES BETWEEN TAURINE AND

INDICINE 86

Abstract 86

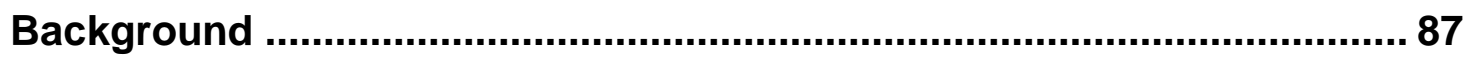

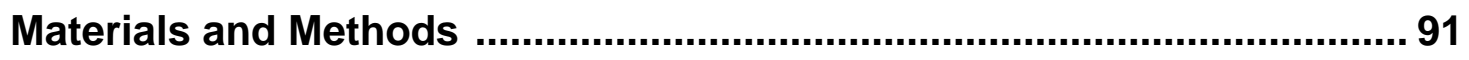

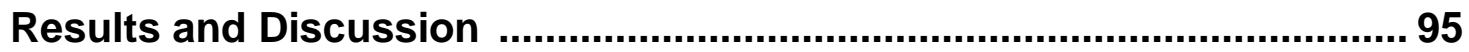

Effect of Reference Panel Size ........................................................ 97

Genome-Wide Global Ancestry by Individual ...................................... 99

Local Ancestry Estimation by American Breed ................................... 100

Similarities across American breeds ...................................... 100

Differences between the American breeds ............................... 101

Annotated regions significantly diverged form expected Brahman

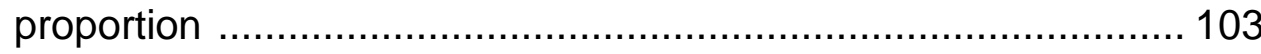

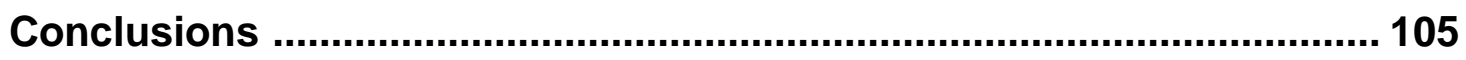

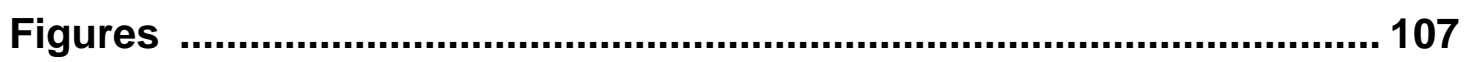


Tables

CHAPTER 4

GENOME-WIDE ASSOCIATION ANALYSIS FOR BREED DIFFERENCES IN FEED EFFICIENCY, GROWTH AND COMPONENT TRAITS 132

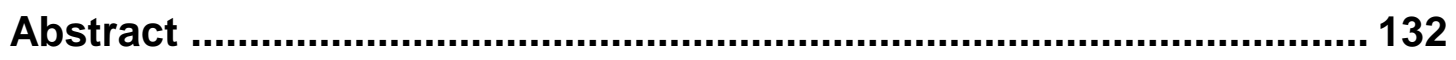

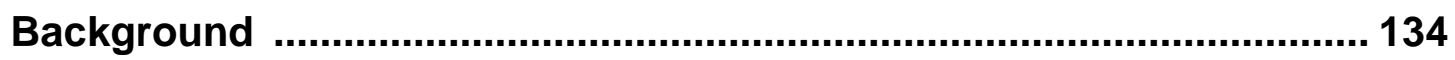

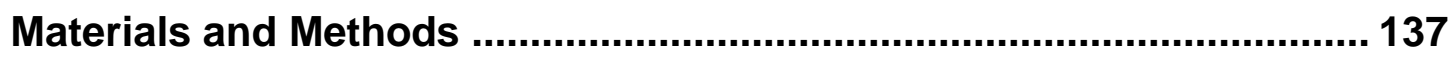

Cattle Populations, Phenotypes and Genotypes ............................... 137

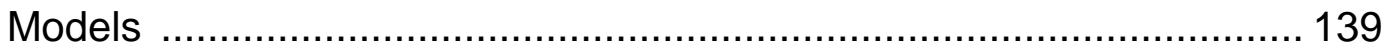

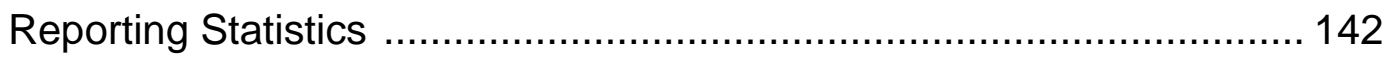

Results and Discussion .................................................................... 143

Significance of Additive Breed Effects (Model 2) ................................ 144

Significance of Heterotic Effects (Model 3) ....................................... 145

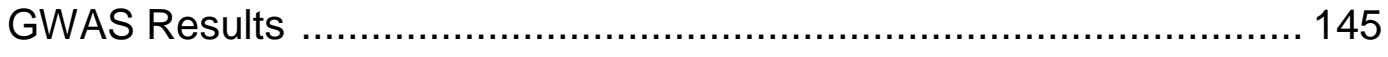

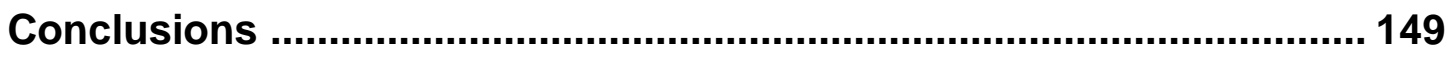

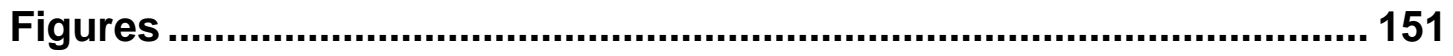

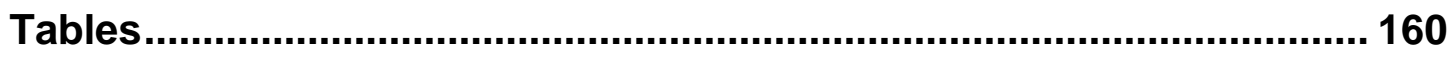

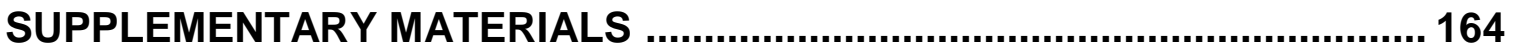

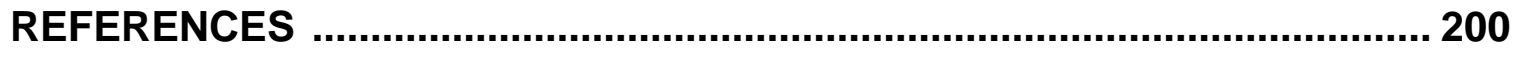

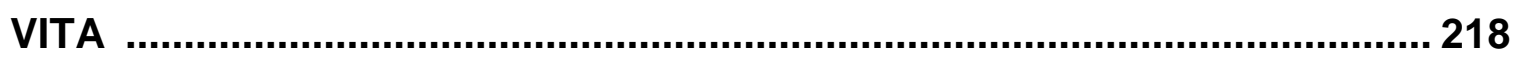




\section{LIST OF FIGURES}

Figure Page

2.1 Flow diagram of the breed composition pipeline ................................. 60

2.2 FastSTRUCTURE results for a random sample of $\leq 200$ individuals per breed from the pool of 17,852 potential reference individuals at $K=19 \quad \ldots 61$

2.3 SNPweights self-assignment analysis results for reference panel sample sets consisting of: (a) $\leq 100$ individuals per breed, or (b) $\leq 50$ individuals

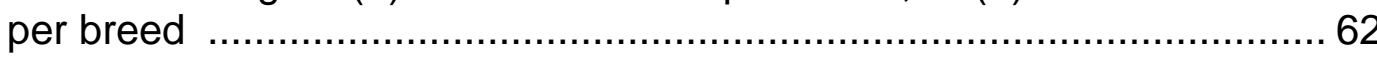

2.4 SNPweights self-assignment of ancestry for candidate reference breed individuals following evaluation of open herdbook breeds using: (a) the $\mathrm{BC} 7 \mathrm{~K}$, or (b) the $\mathrm{BC} 13 \mathrm{~K}$ marker panels

2.5 Reference breed panel constructed by the random sampling of $\leq 50$ individuals per breed from individuals with $\geq 90 \%$ ancestry was selfassigned to reference breed ancestry using the BC7K marker set

2.6 Reference breed panel constructed by the random sampling of $\leq 50$ individuals per breed from individuals with $\geq 85 \%$ ancestry was selfassigned to reference breed ancestry using the BC7K marker set

2.7 Reference breed panel constructed by the independent random sampling of a second sample of $\leq 50$ individuals per breed from individuals with $\geq 85 \%$ ancestry after eliminating individuals represented in the first sample was self-assigned to reference breed ancestry using the BC7K marker set

2.8 (a) Distribution by breed of SNPweights ancestry assignment results for 2,408 registered fullblood animals from open herdbook breeds. (b) Pictorial representation of CRUMBLER estimates for 2,408 registered fullblood animals from open herdbook breeds

2.9 (a) SNPweights ancestry results for 2,005 crossbred Hereford individuals with a-priori breed composition estimates determined by pedigree. (b) Breed assignment reference breed key. (c) Hereford SNPweights estimated proportions using CRUMBLER are plotted against the pedigree estimates 68

2.10 (a) SNPweights ancestry results for 238 crossbred individuals with a-priori breed composition estimates of 50\% Angus and 50\% Simmental based on a reference panel with $\leq 50$ individuals per breed sampled from individuals with $\geq 85 \%$ assignment to their breed of registry. (b) Breed assignment for the crossbred individuals can be determined using this reference breed key 
2.11 Genotypes were simulated for the indicated number of generations of random mating, with generation 1 (G1) animals being 50:50 proportion except when two parents from the same breed were mated. SNPweights results were obtained using CRUMBLER pipeline parameters correlations between these estimates and the known simulated breed compositions were produced and the proportion of individuals within each correlation class is indicated

2.12 (a) SNPweights ancestry results using CRUMBLER pipeline for 11,362 Brangus, 3,832 Beefmaster, and 2,010 Santa Gertrudis individuals. (b) Breed assignment for these advanced generation composite animals can be determined using this reference breed key

2.13 Self-assignment of ancestry for the animals in the reference breed set formed with $\leq 50$ individuals per breed from the individuals that had $\geq 85 \%$ assignment to their breed of registration using ADMIXTURE

2.14 ADMIXTURE analysis conducted using the same data as shown in Figure 2.13 (first four rows) merged with an additional 2,005 high percentage crossbred Hereford target individuals (last row)

2.15 ADMIXTURE analysis conducted using the same data as shown in Figure 2.14, where the 2,005 Hereford crossbred individuals appear before the reference individuals in the input genotype file 74

2.16 ADMIXTURE analysis conducted using the same data as shown in Figures 2.13 and 2.14, but with the order of the individuals in the input genotype file randomized

2.17 Reference breed panel constructed by the random sampling of $\leq 50$ individuals per breed from individuals with $\geq 85 \%$ ancestry was selfassigned to reference breed ancestry using the BC6K marker set

3.1 Brangus most likely assignment for the EXPANDED reference ........... 107

3.2 Beefmaster most likely assignment for the EXPANDED reference ....... 108

3.3 Santa Gertrudis most likely assignment for the EXPANDED reference 109

3.4 Brangus most likely assignment for the CRUMBLER reference .......... 110

3.5 Beefmaster most likely assignment for the CRUMBLER reference ...... 111

3.6 Santa Gertrudis most likely assignment for the CRUMBLER reference 112 
3.7 Brangus ancestry estimation by individual ..................................... 113

3.8 Beefmaster ancestry estimation by individual ................................. 114

3.9 Santa Gertrudis ancestry estimation by individual ............................ 115

3.10 Estimated Brangus ancestry by chromosome ................................... 116

3.11 Estimated Beefmaster ancestry by chromosome ….......................... 117

3.12 Estimated Santa Gertrudis ancestry by chromosome ….................... 118

3.13 Significant regions of deviation from genome-wide Brahman proportion for Brangus by chromosome ............................................................... 119

3.14 Significant regions of deviation from genome-wide Brahman proportion for Beefmaster by chromosome ........................................................ 120

3.15 Significant regions of deviation from genome-wide Brahman proportion for Santa Gertrudis by chromosome ...................................................... 121

4.1 Distributions of normalized CRUMBLER breed composition estimates for FE individuals ......................................................................... 151

4.2 Manhattan plots of GEMMA GWAS for average daily gain ................. 152

4.3 Manhattan plots of GEMMA GWAS for dry matter intake ................... 153

4.4 Manhattan plots of GEMMA GWAS for metabolic mid-weight ............. 154

4.5 Manhattan plots of GEMMA GWAS for residual feed intake ............... 155

4.6 Q-Q plot of GEMMA GWAS for dry matter intake ............................. 156

4.7 Q-Q plot of GEMMA GWAS for average daily gain ......................... 157

4.8 Q-Q plot of GEMMA GWAS for metabolic mid-weight ....................... 158

4.9 Q-Q plot of GEMMA GWAS for residual feed intake .......................... 159 
Table

\section{LIST OF TABLES}

2.1 Genotype data for 48,776 registered individuals from 20 breeds were used to establish the reference population …………................................. 77

2.2 The number of variants queried by each assay and the number of individuals from the 20 reference breeds genotyped using each assay .. 79

2.3 Number of individuals for each reference breed assigned to their breed of registration by minimum ancestry threshold 80

2.4 Ancestry proportion statistics for the self-assignment of reference panel members from samples of $\leq 50$ or $\leq 100$ individuals from the candidate reference breed individuals

2.5 Average predicted ancestry and variance in predicted ancestry for candidate reference breed individuals when filtered on minimum predicted ancestry

2.6 Average breed ancestry percentages assigned to American Breed individuals

3.1 Genotype data for American breeds

3.2 Genotype data for individuals from 5 breeds used to generate the reference population

3.3 Proportion of haplotypes by chromosome for each American breed with marginal probabilities of most likely breed assignment by RFmix between 0.05 and 0.95

3.4 Average ancestry to reference populations throughout the genomes for each of the American breeds

3.5 Chromosome 5 Brahman proportion diverged regions and previously published associations

3.6 Brahman proportion diverged regions and previously published associations for Beefmaster

3.7 Brahman proportion diverged regions and previously published associations for Brangus

3.8 Brahman proportion diverged regions and previously published associations for Santa Gertrudis 
4.1 Genotype data for feed efficiency animals by assay ......................... 160

4.2 Mean and standard deviation of CRUMBLER breed assignments for FE

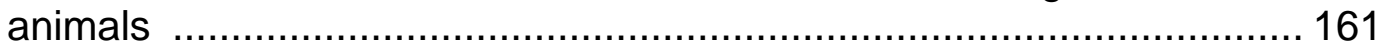

4.3 Significance between models using likelihood ratio tests .................... 162

4.4 Variance component and pseudo heritability estimates for feed efficiency and component traits 


\section{LIST OF SUPPLEMENTARY FIGURES}

Figure

S2.1 An overview of the processes and iterations of filtering conducted in the development of the reference panel 168

S2.2 Preliminary fastSTRUCTURE analysis of candidate Angus and Simmental reference population animals 169

S2.3 Preliminary fastSTRUCTURE analysis of candidate Angus and Gelbvieh reference population animals 170

S2.4 Preliminary fastSTRUCTURE analysis of candidate Angus and Limousin reference population animals

S2.5 Preliminary fastSTRUCTURE analysis of candidate Angus and Red Angus reference population animals 172

S2.6 Preliminary fastSTRUCTURE analysis of candidate Red Angus, Hereford, Shorthorn and Salers reference population animals 173

S2.7 Preliminary fastSTRUCTURE analysis of candidate Red Angus, Hereford and Shorthorn reference population animals 174

S2.8 Preliminary fastSTRUCTURE analysis of candidate N'Dama, Nelore and Brahman reference population animals 175

S2.9 SNPweights self-assignment analysis for the reference sample set containing $\leq 200$ individuals per breed analyzed using the BC7K marker set

S2.10 SNPweights self-assignment analysis for the reference sample set containing $\leq 150$ individuals per breed analyzed using the BC7K marker set

S2.11 SNPweights self-assignment analysis for the reference sample set containing $\leq 50$ individuals per breed analyzed using the BC7K marker set

S2.12 SNPweights self-assignment analysis for the reference sample sets containing $\leq 50$ individuals per breed analyzed using the BC13K marker set

S2.13 SNPweights self-assignment analysis for the reference sample set with $\geq 80 \%$ ancestry to breed of registry and $\leq 50$ individuals per breed using the $\mathrm{BC} 7 \mathrm{~K}$ marker set 
S2.14 SNPweights self-assignment analysis for reference sample set with $\geq 75 \%$ ancestry to breed of registry and $\leq 50$ individuals per breed using the BC7K marker set

S2.15 SNPweights self-assignment analysis for the reference sample set with $\geq 70 \%$ ancestry to breed of registry and $\leq 50$ individuals per breed using the BC7K marker set 182

S2.16 SNPweights self-assignment analyses using a reference panel with $\leq 50$ individuals per breed and sampling from the individuals with $\geq 85 \%$ assignment to their breed of registry but with (a) Red Angus or (b) Angus excluded from the reference panel 183

S3.1 Brangus ancestry estimation by individual (prior to outlier removal) $\ldots . .184$

S3.2 Beefmaster ancestry estimation by individual (prior to outlier removal)

S3.3 Santa Gertrudis ancestry estimation by individual (prior to outlier removal)

S3.4 Distribution of marginal probabilities of foundation breed assignment for Brangus

S3.5 Distribution of marginal probabilities of foundation breed assignment for Beefmaster 188

S3.6 Distribution of marginal probabilities of foundation breed assignment for Santa Gertrudis 189

S3.7 Brangus reference panel comparison ............................................... 190

S3.8 Beefmaster reference panel comparison ........................................... 191

S3.9 Santa Gertrudis reference panel comparison ................................... 192 


\section{LIST OF SUPPLEMENTARY TABLES}

Table Page

S3.1 Most significant diverged regions from Brahman expectation for each

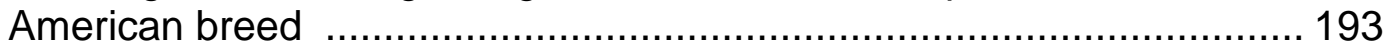

S4.1 PVE, log-likelihood at MLE, and effect estimates for Model 1 by trait ... 195

S4.2 PVE, MLE log-likelihood at MLE, and effect estimates for Model 2 by

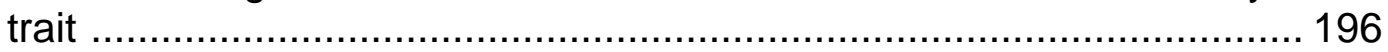

S4.3 PVE, MLE log-likelihood at MLE, and effect estimates for Model 3 by

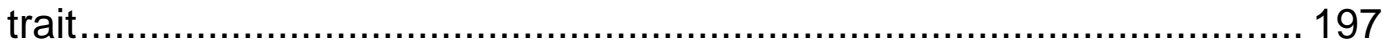

S4.4 Genomic inflation factors associated with Q-Q plot $p$-values ................ 199 


\section{CHAPTER 1}

\section{APPLICATIONS OF ANCESTRY ESTIMATION IN CATTLE}

\section{Abstract}

Accounting for ancestry and cryptic population structure is important in population genetic data analysis. The estimation of ancestry can help elucidate the effects of admixture or stratification on allele and genotype frequencies caused by population substructure. Human population genetics has examined these issues in detail and has developed models which incorporate ancestry effects in the estimation of population susceptibilities to disease risk in association analyses, as well as for the interpretation of genotype disease risks in admixed individuals for use in personalized medicine. In livestock, allele frequencies frequently differ between breeds and these breeds frequently also differ for phenotypes of interest. If these population differences are not accounted for in the analysis, the differences in allele frequencies due to population structure can lead to erroneous associations of loci with phenotypes. The effects of domestication and breed formation in shaping the cattle genome along with the rampant use of crossbreeding to form admixed individuals in the modern beef cattle industry provides an opportunity to evaluate the impacts of breed differences on association analyses of economically relevant traits such as the conversion of feed to weight gain and disease susceptibility. Here, we review the methods used for ancestry estimation and the impacts of ancestry prediction in human population genetic analyses and opportunities for use of these techniques 
in cattle, and more generally in livestock populations. The incorporation of accurate genomic-based predictions of breed composition could fine-tune the resolution of mapping studies targeting the identification of causal loci for livestock species.

\section{Background to Genetic Ancestry}

Accounting for ancestry and cryptic structure present in population genetic data has become an important component of many human genome-wide association analyses (GWAA) to enable corrections to be performed, if necessary, for ethnic or population differences in allele frequencies and means for health-related phenotypes [1]. Differences in allele frequencies between populations occur pervasively throughout the genome and occur at loci that are unrelated to those with causal effects on health-related phenotypes leading to inflated rates of false positive associations between genetic markers and phenotype in GWAA [2].

The utility of GWAA for the discovery of variants of large effect influencing phenotypes has made the use of genome-wide single nucleotide polymorphism (SNP) arrays common in human and livestock and has provided data sources for use in the estimation of population structure and ancestry [3]. Population substructure is not the only source of confounding that leads to spurious GWAA associations, but once the effects of genotyping artifacts and statistical threshold used for GWAA are taken into account, the most common cause of false associations is unaccounted genetic substructure [2]. 
Six issues generally underlie the application of global ancestry estimation, the: (1) detection of population structure, (2) determination of the number of subpopulations that are present, (3) clustering of individuals into subpopulations, (4) determination of the number of ancestral populations that are represented in a population of admixed individuals, (5) assignment of ancestral population proportions to admixed individuals, and (6) identification of the ancestry of chromosomal segments within an individual $[1,4]$.

Several statistical approaches exist for estimating ancestry using SNP data that address these 6 population genetic problems, but unfortunately there is not a single unified software package that optimally addresses all of these issues. Previous reviews have discussed each of these problems and the software that is available to address each genetic ancestry problem [1]. Here, we focus on the applications of ancestry estimation and the detection of population stratification in cattle genetic analyses, and more generally, other livestock species. As a preface, we first discuss the impacts of certain methods and advances in ancestry estimation in human. We next describe challenges posed by the processes of cattle domestication, breed formation, and the modern U.S. beef cattle industry structure as they relate to population genetics. Finally, we describe past and future areas of application of genetic ancestry tools and methods used in livestock population genetics and their potential impacts on modern livestock production. 


\section{Ancestry Prediction in Humans}

Unlike livestock populations where "breed" composition is often determined by a breeder's visual evaluation, or more accurately using pedigree records kept by the breeder, human population genetics is complicated by the social constructs of ethnicity or race when ancestry and population stratification is to be determined from a genetic data set. Ethnicity is self-identified and may have no biological meaning for an individual, and as a result, ancestry or racial composition should not be confused with ethnicity [5]. When phenotypes are collected for human studies, subjects are frequently asked to self-identify with an

ethnic group. However, since self-identified ethnicity may be only weakly associated with individuals' genome composition, incorporating human selfidentified ethnicity to control for population structure in GWAA can, in turn, result in misclassifications of data [6]. Consequently, ethnic identification does not account for the variation in racial admixture that exists among individuals within ethnic groups [5]. This lack of precision can complicate prognoses and risk predictions for admixed individuals in human disease susceptibility and health studies.

Accounting for population stratification in GWAA, can reduce false associations when markers differ in allele frequencies across subpopulations that differ in mean phenotype $[7,8]$. For example without controlling for population structure, lactase (LCT) and interferon regulatory factor 4 (IRF4) would have falsely been identified as being associated with rheumatoid arthritis in Europeans [9]. 
Association studies can be affected by violations of the assumptions that the population under study is panmictic and homogeneous [7]. In a conscious effort to reduce the number of spurious associations and misleading disease associated loci, human association studies have tended to restrict their focus to a single self-identified ethnic group in an attempt to achieve population homogeneity [10]. For example, European Americans have been extensively studied in U.S. GWAA and are often treated as being a genetically homogenous group (as are African Americans and Latinos) $[8,10]$. The use of selfidentification labels such as "white" or "Caucasian" to describe European Americans does nothing to dispel the illusion of genetic homogeneity [10]. Nevertheless, studies have shown that even these panmictic populations which are assumed to possess low levels of admixture can still produce false associations. Campbell et al. (2005) were the first to identify inflated rates of association due to population stratification in a study of a presumed homogeneous European American ancestry population [8]. However, both height and lactase persistence $[11,12]$ were found to be associated with grandparental ancestry which revealed a northwestern-to-southeastern cline across Europe for both traits [8]. This study, as well as a similar finding in the presumed homogenous Icelandic population [7], identified the need for more sensitive measures for detecting stratification in populations that were not excessively admixed [8]. 
While the study of genetically homogenous populations reduces the risk of false discovery, it also results in a reduction of genetic heterogeneity for the traits under study which potentially limits the breadth of interpretation of the study results. Complex admixed populations present unique opportunities to understand the nature of genetic associations that lead to population differences in health and medical outcomes in humans. [13]. Admixed populations arise when individuals from two or more isolated and genetically homogeneous populations interbreed, these original isolated populations are referred to as ancestral or parental populations which evolved independently due to selection, drift and mutation without substantial migration in times past [14]. On the other hand, the diverse racial ancestry of contemporary Hispanics, for example, when leveraged appropriately can be beneficial to the study of complex diseases, such as Chronic Obstructive Pulmonary Disease (COPD) when appropriately modelled and analyzed. However, many of the studies of COPD susceptibility, diagnosis, and treatment in Hispanics have made the unfortunate assumption that Hispanics are a genetically homogenous group and these studies frequently fail to control for population stratification, even when there is significant evidence for the existence of structure within Hispanics [15]. One method to leverage the information inherent to admixed populations when there are genetic variants that differ in frequency between the ancestral populations and when these populations exhibit unique protection or susceptibility patterns for common diseases is admixture mapping $[13,15]$. 
Admixture mapping can be used for the detection of disease-causing variants that are at high frequency within certain of the ancestral populations since the recent admixture causes these alleles to segregate at intermediate frequencies in the recently admixed population $[14,16]$. Admixture mapping scans the genomes of individuals from populations with mixed ancestry to identify local ancestry, regions where the frequency of DNA inherited from either ancestral population differs from the expected genome-wide average between the cases and controls $[16,17]$. Admixture mapping capitalizes on the long-range linkage disequilibrium that exists in admixed populations and because of the large size of introgressed haplotypes, can identify ancestry differences between individuals in a population using a relatively small number of genetic markers [17]. There are several advantages to admixture mapping including: (1) it has a high statistical power when distributions of genotypes and phenotypes differ greatly between the ancestral populations, (2) the SNP density required for a genome-wide admixture map scan is significantly less than for a typical GWAA experiment, and (3) it captures genetic heterogeneity thereby reducing false positive associations [18]. Admixture mapping has enabled the localization of loci affecting human susceptibility to diseases $[13,15]$. In the case of prostate cancer, the most common noncutaneous malignancy among U.S. men, there is a $\sim 1.6$-fold higher prevalence among African American men than among European American men [16]. This suggests that different genetic factors may underlie the variation in susceptibility between the two populations. In fact, Freedman et al. (2006) reported that men with African, rather than European, ancestry at locus "8q24", 
have an increased risk for prostate cancer prior to 72 years of age. Because disease susceptibility differences exist between populations with different racial ancestries, the identification of ancestry proportions in admixed individuals, rather than the ethnic group with which they identify is important. As a result, direct estimates of ancestry composition based on genotype data, or genomic ancestry, are essential to minimize the effects of population stratification on association analyses [6]. Linkage analysis has also been used to map disease risk loci in admixed populations but has a low resolution for the identification of genomic regions harboring these loci due to the effects of recombination over a limited number of generations, whereas admixture mapping can detect small haplotypes reflecting admixture that may have occurred as much as 100 generations ago [14]. However, admixture mapping is not a powerful tool for the identification of genetic risk loci when the ancestral populations represented in the admixed individuals do not significantly differ in allele frequencies at disease loci or do not differ for their disease prevalence.

In GWAA where data from hundreds of thousands of markers are used to evaluate associations with a phenotype, methods for controlling for population stratification are common. However, to detect and control for the effects of population stratification in replication, candidate gene, or fine mapping studies which may use only a small number of makers, an approach using small subsets of ancestry informative markers (AIMs) is highly desirable $[19,20]$. Validated AIM SNP panels have been developed, with as few as 64 SNPs, and have been 
successfully used to characterized sample sets from diverse population groups $[20,21]$. Because AIM panels are often constructed and validated using data for a small set of populations, consideration should be given to the selection of the AIM panel that best suits the data sample. AIM panels selected to evaluate ancestry should vary based on the study and be dependent upon available knowledge pertaining to the evolutionary history of the sampled population [22]. AIM panels can be used to assign individuals to specific ancestral groups or to account for allele frequency differences between ancestral groups in both the cases and controls in the analysis of any phenotype.

The advent of high-throughput genotyping technologies, as well as the declining cost of SNP arrays, presents an opportunity for the evaluation of ancestry using denser marker sets than those represented in AIM panels. GWAA SNP assay data are often used for ancestry estimation in two ways: (1) the number of SNPs is reduced to those previously used in an AIM panel to estimate global ancestry proportions, or (2) to predict local ancestry genome-wide based on the use of a sliding window of overlapping SNPs [23]. Genetically homogeneous populations were initially the focus of GWAAs, however, as previously discussed, admixed populations provide opportunities for identifying genetic variation that may have previously been missed due to the near fixation of alleles within homogeneous populations. With the incorporation of ancestry estimates, admixed populations can be used to reveal novel genes or loci for traits of interest without the confounding effects of unaccounted structure in the data. In some cases, 
GWAAs have been used to identify SNPs near genes associated with a phenotype and admixture mapping within diverse populations has then been used to identify additional novel variants or to confirm the GWAA associated SNPs [24]. Admixture mapping, although able to detect admixture events occurring up to 100 generations in the past, does not possess the level of resolution for causal variant detection that can be obtained through association mapping $[14,25,26]$. Association mapping capitalizes on all of the recombination events that have occurred back to the most recent common ancestor which may be many generations in the past [14]. Consequently, association mapping is the method of choice for studying complex trait inheritance patterns by identifying markers with significantly diverged allelefrequencies between the "case" and "control" populations [26]. In spite of the differences in resolution, the two methods are complementary. Association mapping searches for genomic locations where allele-frequencies differ between cases and controls, whereas admixture mapping scans for genomic regions that are diverged from ancestral proportion expectation in the cases, but not controls [25]. Admixture mapping tests for differences in phenotype that are due to alleles that differ by ancestry, while association studies test for genotype-phenotype associations regardless of the ancestry of alleles [14]. Pino-Yanes et al. (2015) combined GWAA and admixture mapping in an attempt to explain the missing heritability of $\lg E$ expression, a class of antibody that is strongly associated with allergic disorders and asthma severity in Latinos, a highly admixed and genetically diverse population. Previously, Latinos had not been significantly 
represented in IgE GWAA studies, thus creating an opportunity for the identification of novel associations. Pino-Yanes et al. (2015) conducted a GWAA, accounting for global, genome-wide, African and Native American ancestry proportions, and were able to confirm previously associated loci as well as identify a novel genome-wide association with total $\lg E$ levels. They next performed the first admixture mapping analysis of IgE levels and identified a significant association in the $\mathrm{MHC}$ region associated with African American ancestry. The combination of approaches proved to be highly effective, due to the diversity within Latinos, and the admixture mapping captured additional important local ancestry genetic variation that had not previously been identified in conventional GWAAs for IgE [24].

Other scenarios exist in which the a priori classification of ancestry are required to: (1) explain properties of populations based on a sample of individuals without regard to any specific phenotype, or (2) classify a set of individuals of unknown origin or identity [4]. Several software packages have been developed for this purpose including STRUCTURE and ADMIXTURE which use multi-locus data [4, 27]. These analyses can use high density marker assays for ancestry estimation or AIM panels trained and validated for the populations likely to have contributed to the ancestry of these individuals.

The availability of diverse genetic variation profiles cataloged by the International HapMap Project [28] and the 1000 Genomes Project [29] provided an avenue for 
association studies to use larger SNP panels through a process of imputation to infer genotypes at untyped SNPs. Causal SNP are unlikely to be directly genotyped by SNP genotyping assays, however, due to the presence of linkage disequilibrium, associations between interrogated SNPs and phenotypes can still be detected. Through the use of typed SNPs and knowledge concerning the haplotypes present within a population, imputation methods can be used to infer genotypes at SNPs that are not represented on genotyping assays to increase the resolution of GWAA and fine-mapping studies [3]. Following imputation, using whole genome-sequence SNP data from the 1000 Genomes Project [29], $10,421,983$ SNPs were used to identify novel loci associated with alcohol dependence in European Americans, including the first associations demonstrated in African American populations [30]. Additionally, Pino-Yanes et al. (2015) capitalized on the ability to impute genotypes to perform fine-mapping within candidate regions associated with IgE levels that had been identified by GWAA and admixture mapping. Fine-mapping these regions increased the resolution of association detection and also the ability to detect novel associations within the Latino population [24]. However, the key to accurate genotype imputation is a well-matched reference panel of haplotypes that provides pertinent information enabling the estimation of genotypes at untyped loci in the imputed individuals. As was expected, the highest imputation accuracy was achieved when the reference haplotypes were matched to the ancestral or ethnic backgrounds represented in the studied individuals [31]. Consequently, the use of haplotypes estimated from ancestral samples that do 
not fully represent the ancestry of the individuals within the studied population can significantly reduce imputation accuracy. To avoid the mis-representation of individuals by making a priori assumptions about the populations that should be included in the reference set, large pools of reference haplotypes are created, representing all possible ancestral backgrounds $[32,33]$. Such reference panels require large amounts of sequence data on large numbers of individuals representing the ancestral populations. Human genomes are mosaic in nature and different genomic regions may have different ancestral backgrounds [34, 35]. Contemporary human populations, in general, do not originate from a single ancestral population. Consequently, reference panel construction should be based on the creation of local ancestry matched reference haplotype panels, which will increase the accuracy of imputation [36]. With this approach, ancestry is estimated for each locus within each sample and this information is used to inform the imputation of genotypes [36]. For example, both chromosomes at chr3:12,345,000-13,456,000 in a specific individual were determined by local ancestry estimation to be derived from population A, only the reference haplotypes from population A would be used to impute genotypes in this genomic region for this individual.

Methods for the estimation of genetic ancestry in human populations have undergone considerable evolution. The study of admixed human populations presents a unique opportunity to identify large-effect loci responsible for variation in health phenotypes, which brings us closer to the implementation of 
personalized medicine and treatment breakthroughs that will improve the wellbeing of all humans. To escalate this objective, the human population genetics field has created, tested, and validated methodologies and software that can be applied to other species, such as cattle or livestock populations, in general.

\section{Domestication of Cattle}

Population structure and genetic ancestry are implicit to the study, interpretation, and application of population genetics in cattle. The domestication of cattle, and the structure of the modern cattle industry, have greatly contributed to shaping the nature of the variation that exists within cattle populations and it is valuable to have a working knowledge of the two. The two currently recognized subspecies of cattle, Bos taurus taurus and Bos taurus indicus, arose from two independent domestication events in the Fertile Crescent and Indus Valley, respectively, approximately 10,000 years ago. The migration of humans from these regions into Europe and Asia led to the formation of geographically isolated populations of cattle, and ultimately to the formation of distinct breeds of cattle about 200 years ago $[37,38]$. Breeds of cattle were initially developed by the selection of individuals for distinct coat colors, the presence or absence of horns, and specialized performance for draft, meat or milk production. During and following the migration of cattle, new germplasm was often introduced into local cattle populations by hybridization with local wild aurochs or other migrating cattle. This initial admixture provided the basis for the subsequently distinct and isolated populations that would later be developed into breeds [38]. For example, while 
breeds such as Friesians and Shorthorns developed without any recorded histories of crossbreeding, the Belgian Blue breed was formed between 18401890 by crossing local cattle with imported Friesians and Shorthorns [37].

Crossbreeding was extensively used prior to the formation of cattle breeds to capitalize on desirable characteristics of cattle from different geographic regions of the world. Humpless cattle, Bos t. taurus, and humped cattle, Bos t. indicus, were extensively crossed to produce hybrid individuals that were well suited to the environments and production systems of Asia, Africa, and North and South America [38]. Asian cattle reveal the complicated nature of the process of domestication. Asian cattle breeds originated from the hybridization of domesticated species and subspecies from the Indian subcontinent (B. t. indicus and Bos frontalis) and from migrating B. t. taurus cattle from the Fertile Crescent and Europe. Admixture in many regions across Asia occurred between domesticated B. t. indicus and B. t. taurus. Additionally, some populations were admixed with other domesticated species, including water buffalo (Bubalus bubalis) and yak (Bos grunniens) contributing to even higher levels of genetic diversity within these populations [38]. Throughout history, the formation of cattle populations has been influenced by bottlenecks, co-ancestry, migration, and admixture events [37]. Domestication, exportation, and breed formation have affected the variation present within the cattle genome, both within and across breeds and populations. Historical crossbreeding and admixture events within and between cattle populations can complicate population genetic studies 
performed within the species, particularly if ancestry differences are not properly accounted for in the analyses [38].

\section{Modern Cattle Industry}

The development of cattle breeds about 200 years ago came with the formation of registries and breed associations that were created to assist producers with breed management practices, such as pedigree recording. Fullblood cattle have pedigrees that can exclusively be traced back to breed founder animals in the herdbook registries for their respective breeds which are generally managed within each country, including the U.S. For fullblood animals, the primary breed composition of the genome is identified by its registration by a specific breed association. For inclusion within the breed association's registry, both parents of the animal must be identified and registered with the association and, for the last 50 years, the nominated pedigree must be validated using blood or DNA typing. For breed associations with closed herdbooks, all animals are fullblood and, in theory, the pedigrees of all registered animals can be traced back to the breed's foundation animals. On the other hand, breeds with open herdbooks allow the registration of fullblood animals and crossbred animals once they reach a certain expected genome composition for the breed such as $31 / 32^{\text {nds }}$ through a "grading up" process. Grading up consists of strategic crossbreeding to obtain the required percentage of their genome, based upon pedigree records and parentage validation, originating from the respective breed and these animals are referred to as purebreds. 
Over the last 50 years, parentage validation through blood typing or DNA analysis via microsatellite or SNP typing, has been used by breed associations to validate nominated pedigrees and identify pedigree errors. Pedigree errors have varying degrees of effect on an animal's breed composition but can lead to an admixed animal being classified as fullblood and subsequent incorrectly estimated admixture proportions based upon pedigree in descendant purebred animals. These errors persist in breed herdbooks either due to their infiltration before the implementation of blood or DNA typing or the inability of the typing to identify pedigree mistakes. Mendelian sampling, the random assortment of chromosomes into gametes, selection, and pedigree errors, can lead to significant variation in the extent of shared DNA between relatives separated by more than a single meiosis $[39,40]$.

Modern cattle production, particularly U.S. commercial beef production, often capitalizes on the benefits of crossbreeding to capitalize on breed differences for economically important traits and hybrid vigor or heterosis to increase fitness, adaptability, and the overall productivity of animals. Crossbreeding production systems result in herds that can range in breed composition from $F_{1}$ hybrids, with high degrees of heterosis to advanced generation composites which also retain some of the heterosis found in the $F_{1}$ s. Frequently, herds using crossbreeding systems source fullblood or purebred bulls from registered breeds. To avoid the long-term loss of heterosis, a breeder may employ a rotational crossbreeding 
system, in which the breed of the bull is changed each year. Rotational crossbreeding systems result in significant changes in admixture proportions of progeny from year to year. Additionally, a common strategy to improve the adaptability of cattle in tropical and subtropical climates while still maintaining favorable beef production is to cross European taurine cattle with Zebu (B. $t$. indicus) [41, 42] cattle. This strategy has been used to produce new breeds of cattle such as Brangus and Braford in which the taurine component of each animal's genome is expected to be $62.5 \%$ and the indicine component $37.5 \%$. However, Mendelian sampling of parental gametes and selection applied to advanced generation animals could cause significant departures from these expected frequencies. If not accounted for, the underlying ancestry of animals obtained from crossbreeding systems could have substantial impacts on population genetic studies, as well as on the application of genetic technologies, such as genomic prediction [43].

Genomic prediction is the "prediction of genetic merit of selection candidates based on genome-wide marker genotypes using information from a reference population with both phenotypes and genotypes" [44]. Since the introduction of high-density SNP genotyping in 2008, Holstein cattle have become the model population for the development, implementation, and optimization of genetic progress by genomic prediction [45]. Holsteins are the predominant breed of dairy cattle throughout much of the developed world, and have historically been strongly selected for milk production characteristics. Dairy cattle rely heavily on 
the use of artificial insemination globally to capitalize on the genetic superiority of elite bulls. Historically, these superior bulls were progeny tested and "proven" to be elite through the evaluation of the production characteristics of their daughters. Time constraints between the sexual maturity of a bull and the ability to obtain measurements of traits such as fertility and milk production on its daughters made the wide-spread use of younger, unproven bulls a risky decision. However, by 2009, 3,576 young Holstein bulls had been genotyped and genomic prediction estimates were incorporated into their estimates of breeding values to achieve reliabilities of $50 \%$, providing the equivalent accuracy of these bulls having 11 progeny-tested daughters [46]. Seven years following the implementation of genomic prediction in U.S. Holstein cattle, the rates of annual genetic improvement have increased by $50 \%$ to $100 \%$ for moderately heritable traits and by $300 \%$ to $400 \%$ for lowly heritable traits $[45,47]$.

In comparison, the U.S. beef industry has been slower to implement genomic evaluations into their selection and prediction tools. Much of this was due to the diversity and structure of the U.S. beef industry. For example, the limited use of artificial insemination in beef herds contributed to lower extents of genetic connectedness across herds and regions within breeds and lower rates of selection response relative to the U.S. dairy industry. Furthermore, phenotype collection in beef herds is much less extensive than in dairy herds, especially in commercial operations. Finally, there are many more beef breeds than dairy breeds and crossbreeding is extensively used in beef cattle production [48]. 
Accuracies of genomic predictions in National Cattle Evaluations for several beef and dairy cattle breeds is influenced by: (1) effective population size, (2) availability of genotype and phenotype information, and (3) relatedness of reference individuals (the training population) to the selection candidate population [49]. Often, the populations constructed to study the genomics of economically relevant traits, particularly in beef cattle, such as feed conversion efficiency $[50,51]$ or bovine respiratory disease [52] are sourced from commercial operations that likely employ crossbreeding systems. When commercially sourced animals are used, the amount of admixture present in the sampled animals may impact the results of GWAAs $[50,51]$ and downstream genomic prediction models because the specific breed composition of the training population animals is not completely understood. The application and utility of these models in industry populations that differ from the training population is also not understood, leaving much to be desired from an industry genetic improvement perspective.

Many commercial cattle operations do not use breed association registrations and pedigree tracking to facilitate record keeping. As a result, many of these commercial operations use visual evaluation to assign animals to a breed group. In many cases, commercial operations contribute a large proportion of the data that is used for academic research. Much like the inadequacy of self-identified ancestry in humans, the visual classification of cattle based on specific breed characteristics suffers from many problems. Many of the visually evaluated 
breed characteristics are determined by alleles at relatively few loci. For example, Angus cattle are extensively used in crossbreeding systems to produce black hided animals with high growth and carcass merit. A single dominant allele at the MC1R locus masks all other solid coat colors and creates a black hided animal. Thus, black hided animals will generally be classified as being Angus influenced, but the true extent of Angus within the genomes of black hided animals many be very small. The presence of this "cryptic" population structure means that Angus branded beef products in the U.S. may come from animals that actually have little Angus content and this may significantly impact GWAA using these animals [4]. Consequently, the use of SNP genotype data to infer ancestry is highly preferred and could also be applied to breed identification for branded beef or value-added programs. The genomic inference of ancestry would provide a more reliable breed composition estimate when pedigree data are compromised, missing, or inadequate.

\section{Ancestry Prediction in Cattle}

The study of population structure and variation in cattle has long been of interest to elucidate the complexities of the domestication process and species history of cattle. In addition, the analysis of population structure could be used for breed identification, breed-labeled beef products, and value-added programs. Much like the effect that the publication of genome variation resources had for human population genetic research, the publication and availability of draft assemblies of the bovine genome [53], and the release of the Bovine HapMap Consortium 
results [54], propelled the study of population structure in cattle by allowing the full spectrum of genetic diversity to be surveyed in bovines. Early work on the population genetic structure and diversity of cattle using mitochondrial (mt) DNA, Y chromosome, or sparse microsatellite marker data [55-59] transitioned to studies using SNP data that focused on the development of small numbers of ancestry informative markers trained and validated for use within specified populations [60,61]. However, with the declining cost of high-throughput genotyping technologies, genome-wide high-density SNP assays now provide more robust data for assessing population structure using thousands of markers distributed throughout the bovine genome $[38,54,62,63]$.

\section{Ancestry Informative Markers}

Lewis et al. (2011) were the first to develop a set of ancestry informative markers in cattle based on an unsupervised principal component analysis (PCA) for the classification of individuals into specific breed groups without a priori knowledge of the individuals' breeds [64]. To enable the process of breed assignment, they developed a decision tree to hierarchically classify individuals of unknown ancestry [64]. The highest branches within the decision tree classified individuals into B. t. taurus, B. t. indicus or hybrid, while the lowest branch distinguished between Angus and Red Angus. They identified a set of PCA Informative Markers (PCAIMs), comprising a few hundred SNPs, that could be used to accurately assign individuals to a particular breed or group of breeds based on the decision tree [64]. Using 250-500 SNPs, this hierarchical decision making 
process achieved close to a $100 \%$ prediction accuracy for assigning individual ancestry to fullblood animals. As one might expect, the lowest levels of prediction accuracy occurred when distinguishing between two highly related breeds, such as Angus and Red Angus at the lowest level of the decision tree. Even so, with a carefully selected panel of as few as 50 SNPs, the PCAIM method achieved a 92\% classification accuracy for animals even within closely related breeds [64]. While useful for the classification of fullblood individuals, this classification method only allows the similarity clustering of individuals, rather than generating the ancestry proportion estimates which are useful for downstream analyses. Additionally, each set of PCAIM was trained, tested, and validated within specific nodes of the decision tree and it is unclear what the consequences would be of using a PCAIM marker that was inefficiently matched to the tested individuals (i.e., if a hybrid breed PCAIM was used for evaluation and the tested individuals do not contain any Beefmaster, Santa Gertrudis or Sheko individuals, for example). Consequently, some a priori information must be available concerning the breeds likely to be represented in the tested animals for this approach to yield useful results. And again, the approach is not well suited to the classification of animals with varying degrees of admixture in their ancestries.

\section{Global Ancestry of Admixed Populations}

The declining cost of high-throughput genotyping technologies has provided the opportunity to infer breed composition using high-density genome-wide SNP 
panels. Global ancestry estimation, using methods such as STRUCTURE [4], Admixture [27] or fastSTRUCTURE [65] enables the incorporation of estimates of breed composition, rather than breed clustering, into GWAA models to control for potential breed differences within the phenotyped individuals. Nevertheless, the use of smaller sets of markers for global ancestry estimation is advantageous in terms of both time and computational costs [66]. This frequently involves the filtering of high-density SNP data for markers that have previously been identified as highly informative or subsetting markers that are common to a wide range of genotyping platforms to enable a broader application of the analyses. As the number of genotyped cattle increases, the ability to develop reference panels of individuals that are representative of target breeds has become feasible. The development of reference panels is technically difficult, as the extent of genetic diversity within each breed should be captured by the individuals in the reference panel. Crum et al. (2019) developed a robust analysis pipeline, CRUMBLER, that streamlines the global ancestry estimation process and uses SNP weights from a predetermined reference panel of 806 animals representing 17 breeds of cattle that are common in the U.S. The reference panel of SNP weights was based on a subset of 6,799 markers that are common to many of the commercially available SNP genotyping assays and can be used to infer the ancestry and breed composition of individuals with completely unknown ancestries $[67,68]$. 


\section{Local Ancestry of Admixed Populations}

For global ancestry estimation, it is implicitly assumed that ancestry proportions from each of the pure populations are consistent throughout the genome. However, sources of variation such as genetic drift, random sampling of parental alleles, and selection can cause changes in the local ancestry of an individual. While genetic drift tends to affect the entire genome of an individual, selection targets only specific functional elements that are correlated with the selected trait. Strong recurrent selection is often polygenic and can be detected throughout the genome in regions subjected to selective sweeps. These selective sweep regions are often associated with large effect variants and are identified by a loss of haplotypic variation within the chromosomal region surrounding the selected variant [69]. In admixed individuals, selected alleles will have higher frequencies following generations of directional selection, resulting in genomic regions that deviate from the genome-wide average ancestry [70]. Such regions likely contain alleles associated with favorable phenotypes that originated from one, or perhaps more, of the pure ancestral populations.

The demographic history of cattle presents a unique opportunity for admixture mapping, a local ancestry tool widely used in human population studies but not as popular in other organisms. The genomes of B.t.taurus and B. t. indicus are estimated to have diverged 56,000 generations ago, a significantly higher

divergence time than that for African and European human ancestry [18]. Considering the novel and causal disease risk loci that have been identified as a 
result of admixture mapping in human populations, notably African American populations $[16,17,71]$, this divergence between cattle subspecies presents an opportunity for similar successes. The first application of admixture mapping in cattle was conducted for susceptibility to tuberculosis and coat color pigmentation using a panel of 662 evenly spaced autosomal SNPs identified for high allele frequency divergence in Ethiopian hybrid cattle [18]. The admixture map scan revealed genomic regions that corresponded to known loci for pigmentation, $M C 1 R$, and tuberculosis susceptibility, the TLR cluster on chromosome 6 . The history of recent admixture in the population and low marker resolution resulted in large genomic intervals, where a number of other genes within the interval could also be considered as candidates [18]. The candidate loci identified for tuberculosis susceptibility in this study did not correspond with previous candidates identified in GWAA in British and Irish cattle herds [72, 73]. However as one might expect, admixture mapping and local ancestry inferences are useful for the detection of candidate loci and regions where the ancestral populations present in the studied admixed individuals may have been close to fixation for alternate causal variants in the fullblood parental breeds [18].

Applications for imputation and fine-mapping of regions identified through methods such as admixture mapping could provide an increased resolution for the detection of causal loci. Additionally, consideration of local ancestry on a genome-wide scale, rather than based on small AIM panels, could also increase the resolution for the detection of variants associated with traits of interest in 
crossbred populations. With the increasing availability of data from high-density SNP genotyping assays in cattle, the need for more precise local ancestry estimates is beginning to be recognized in population genetic studies. Khayatzadeh et al. (2016) explored the variability in local ancestry estimated in Swiss Fleckvieh cattle, a recently admixed population of two taurine cattle breeds - Simmental and Red Holstein-Friesian, using 39,525 SNPs to identify selection signatures. While regions of the genome that differed from genome-wide admixture expectation were found to harbor genes known to be associated with strongly selected traits in the dairy industry, such as milk yield, milk composition, and fertility, the identified admixture selection signals represented large regions. Since there were only a small number of generations since the initial admixture event between the two taurine breeds, the narrowing of selective sweep regions through recombination has not yet occurred [74].

Composite cattle breeds such as Beefmaster or Santa Gertrudis, were formed as crosses between B. t. taurus and B. t. indicus breeds and individuals within these breeds with an advanced number of generations since the initial admixture event present an opportunity to evaluate local ancestry effects. We know from pedigree records and the breeding design used to form the founders of these advanced generation composite cattle what the expected global ancestry of each individual's genome should be. For example, in Beefmaster cattle each individual by pedigree should be $1 / 4$ Shorthorn, $1 / 4$ Hereford, and $1 / 2$ Brahman. However, the effects of strong selection over time have likely caused significant 
departures from these parental breed expectations in selected regions of the genome. Regions with significant departures from parental breed expectations could reveal the selection targets within the admixed individuals that correspond to regions exposed to divergent selection in the parental populations. For example, fixation or near fixation of taurine alleles is expected in regions harboring genes that are associated with meat quality and indicine alleles is expected in regions associated with adaptation. Danish Red Dairy cattle are an admixed population formed by introgression from Holstein and Brown Swiss breeds, and the genomes of these animals have been shaped by humanmediated selection [75]. A local ancestry study of these cattle has revealed genomic signatures that were due to enrichments of alleles from either the Holstein or Brown Swiss. These regions harbored numerous genes affecting traits that have been strongly selected in modern dairy populations that affect calving traits, body conformation and feed efficiency [75]. Local ancestry and admixture mapping has been largely ignored in cattle and has the potential to reveal the loci that are responsible for breed differences via the analysis of advanced generation admixed animals.

\section{Conclusions}

Genetic ancestry prediction at the population and individual levels has long been important for elucidating the population structure that exists in many species. Underlying population structure within a data set can result in erroneous associations between loci and the studied phenotype if that structure is not 
properly represented in the analytical model. The incorporation of genetic ancestry into population genetic studies in humans has provided a model for other species to follow. The domestication process and history of breed development and subsequent crossbreeding in the commercial U.S. beef industry provide a unique opportunity to incorporate genetic ancestry and population structure information into association analyses. As researchers continue to identify genomic regions harboring loci responsible for breed differences in economically relevant traits through association studies capitalizing on ancestry information, future challenges will require addressing local ancestry prediction and best practice imputation methods. Broadening our understanding of the underlying causes of artifacts and the identification of causal variants affecting traits important to the cattle and livestock industries will require the development of technologies to address the efficiency of livestock production, ultimately assisting producers in overcoming the challenges facing modern agriculture. 


\title{
CHAPTER 2
}

\section{CRUMBLER: A TOOL FOR THE PREDICTION OF ANCESTRY IN CATTLE}

\begin{abstract}
Background

In many beef and some dairy production systems, crossbreeding is used to take advantage of breed complementarity and heterosis. Admixed animals are frequently identified by their coat color and body conformation phenotypes, however, without pedigree information it is not possible to identify the expected breed composition of an admixed animal and in the presence of selection, the actual composition may differ from expectation. As the roles of DNA and genotype data become more pervasive in animal agriculture, a systematic method for estimating the breed composition (the proportions of an animal's genome originating from ancestral pure breeds) has utility for a variety of downstream analyses including the estimation of genomic breeding values for crossbred animals, the estimation of quantitative trait locus effects, and heterosis and heterosis retention in advanced generation composite animals. Currently, there is no automated or semi-automated ancestry estimation platform for cattle and the objective of this study was to evaluate the utility of extant public software for ancestry estimation and determine the effects of reference population size and composition and number of utilized single nucleotide polymorphism loci on ancestry estimation. We also sought to develop an analysis pipeline that would simplify this process for members of the livestock genomics research community.
\end{abstract}




\section{Results}

We developed and tested a tool, "CRUMBLER", to estimate the global ancestry of cattle using ADMIXTURE and SNPweights based on a defined reference panel. CRUMBLER, was developed and evaluated in cattle, but is a species agnostic pipeline that facilitates the streamlined estimation of breed composition for individuals with potentially complex ancestries using publicly available global ancestry software and a specified reference population single nucleotide polymorphism dataset. We developed the reference panel from a large cattle genotype data set and breed association pedigree information using iterative analyses to identify fullblood and purebred individuals that were representative of each breed. We also evaluated the numbers of markers necessary for breed composition estimation and simulated genotypes for advanced generation composite animals to evaluate the precision of the developed tool.

\section{Conclusions}

The developed CRUMBLER pipeline extracts a specified subset of genotypes that is common to all current commercially available genotyping platforms, processes these into the file formats required for the analysis software, and predicts admixture proportions using the specified reference population allele frequencies. 


\section{Background}

Estimation of the breed composition of individuals with complex ancestries has utility for estimating breed direct and heterosis effects as well as for the estimation of the additive genetic merit of these individuals. It also has value for identifying the breed composition of training populations used for genomic selection and hence the identification of target breeds in which the developed prediction equations may have some relevance. Visual classification of cattle based on breed characteristics suffers from similar problems as the selfidentification of ancestry in humans [6], as most breed characteristics are determined by alleles at relatively few loci. For example, recent extensive crossing with Angus cattle in the U.S. produces a black hided animal which masks all other solid coat colors found in other breeds and requires only a single dominant allele at the $M C 1 R$ locus. As a consequence, black-hided cattle have a "cryptic" population structure $[4,6]$ and the visual classification of black-hided animals for branded beef programs can result in the marketing of animals with vastly different Angus genome content.

In the U.S. and many other countries, the breed of an animal is associated with its being registered with a breed association which requires that both parents of the animal be identified and also registered with the association. For the previous 50 years, parentage has been validated by each breed association using blood or, more recently, DNA typing. Many breed associations have closed herdbooks which means, in theory, that the pedigrees of all animals can be 
traced back to the animals that founded the breed's herdbook. Other breed associations have open herdbooks, which means that crossbred animals can be registered with the breed if they have been graded up by crossbreeding to purebred status with the expectation that a certain percentage of their genome (e.g., 15/16 ths $)$ originates from the respective breed based upon pedigree records and parentage validation. Pedigree errors that occurred prior to, or that were not identified following the implementation of blood typing and DNA testing, lead to admixed animals being incorrectly classified as fullblood and incorrectly identified admixture proportions in purebred animals. The effects of recombination, random assortment of chromosomes into gametes and selection can also lead to considerable variation in the extent of identity by descent between relatives separated by more than a single meiosis and can also lead to admixture proportions that differ substantially from expectation based on pedigree.

Crossbreeding is extensively used in commercial beef production and in other livestock species production systems to capitalize on the effects of breed complementarity and heterosis resulting in herds of females that may have very complex ancestries that frequently use fullblood or purebred bulls sourced from registered breeders. Changes in the decision as to which breed of bull to use can result in large changes in admixture proportions of replacement cows and marketed steers between years and large differences can occur between herds for the same reason. When commercially sourced animals are used to generate resource populations to study the genomics of economically important traits such 
as feed conversion efficiency $[50,51]$ or bovine respiratory disease [52], the presence of extensive admixture in the phenotyped and genotyped animals may impact the GWAA $[50,51]$ and leads to the training of genomic prediction models in populations for which the breed composition is not understood. As a consequence, the utility of these models in other industry populations, including the registered breeds in which the majority of genetic improvement is generated is also not understood.

As the number of genotyped beef animals has increased, the need to classify the breed composition of these animals has necessitated the development of a precise and accurate method for estimating breed composition in cattle based on single nucleotide polymorphism (SNP) data. Iterative ancestry estimation analyses performed using different software input parameters may identify those that cause output sensitivity and can lead to an interpretation of population structure that is close to the truth [76]. We developed the CRUMBLER analysis pipeline to streamline the genomic estimation of breed composition of crossbred cattle using high-density SNP genotype data, publicly available software, and a reference panel containing genotypes for members of cattle breeds that are numerically important in North America. The CRUMBLER pipeline is species agnostic and could be adapted for breed composition estimation in other species. CRUMBLER and the reference panel data are available on GitHub (https://github.com/tamarcrum/CRUMBLER). This pipeline tool is released under the GNU General Public License. 


\section{Materials and Methods}

\section{Genotype Data}

From among the numerically most important cattle breeds in North America, in terms of their annual numbers of animal registrations, a list was compiled to define the target breeds for reference panel development. Composite breeds, such as Brangus and Braford, were not included in this list due to lack of available genotype data, but the progenitor Angus, Hereford and Brahman breeds were included. Breeds such as N'Dama, representing African taurine, and Nelore and Brahman, representing Bos taurus indicus cattle, were included. We also initially included breeds that were likely to be involved in early crossbreeding of cattle in the U.S. (Texas Longhorn).

From the 170,544 cattle with high-density SNP genotypes stored within the University of Missouri Animal Genomics genotype database, we extracted genotypes for 48,776 animals identified as being registered with one of the numerically important U.S. Breed Associations or belonging to other world breeds. Pedigree data were also obtained for these animals from each of the Breed Associations, where available (Table 2.1). These individuals had been genotyped using at least one of 9 different genotyping platforms currently used internationally to genotype cattle including the GeneSeek (Lincoln, NE) GGP90KT, GGP-F250, GGP-HDV3, GGP-LDV1, GGP-LDV3, and GGP-LDV4 assays, the Illumina (San Diego, CA) BovineHD and BovineSNP50 assays, and the Zoetis (Kalamazoo, MI) i50K assay. The numbers of variants queried by 
each assay and the number of individuals genotyped using each platform are shown in Table 2.2.

\section{Marker Set Determination}

To maximize the utility of the developed breed assignment tool, we identified the intersection set of SNP markers located on the bovine assays for which we had available genotype data (Table 2.2). However, during the process of identifying the animals that would define the breed reference panel, only 16 individuals had been genotyped using the GGP-LDV4 $(n=2)$ and GGP-LDV3 $(n=14)$ assays and no animals had been genotyped using the GGP-LDV1 assay. To retain as many SNP markers as possible for subsequent analysis, we identified the intersection of markers present on the GGP-90KT, GGP-F250, GGP-HDV3, GGP-LDV3, GGP-LDV4, BovineHD, BovineSNP50, and i50K assays. This intersection set included 6,799 SNP markers (BC7K). The intersection of the markers representing 5 assays (GGP-90KT, GGP-F250, GGP-HDV3, BovineHD, and BovineSNP50) was 13,291 markers (BC13K). By removing only the 16 individuals from the breed reference panel that had been genotyped on the GGPLDV3 and GGP-LDV4 assays, we were able to compare ancestry predictions using two marker set densities (BC13K and $\mathrm{BC} 7 \mathrm{~K})$.

\section{Pipeline}

The developed CRUMBLER pipeline integrates the tools and the computational efficiency of publicly available software, PLINK $[77,78]$, EIGENSOFT $[79,80]$ 
and SNPweights [67] to generate ancestry estimates (Figure 2.1). The pipeline integrates the often cumbersome processes of data reformatting and sequentially processing the data using analytical tools to generate ancestry proportions for targeted individuals based on a curated breed reference panel.

\section{PLINK}

PLINK PED formatted genotypes are required as input to the pipeline. PLINK (v1.90b3.31) was used for data filtering and formatting. Genotypes can arise from any of the common bovine genotyping platforms (Table 2.2), provided that a PLINK compatible MAP file is provided for each assay and data produced using only a single genotyping assay is included in each PED file. The pipeline utilizes the PLINK marker filtering tool (--extract) to extract the user-specified marker subset for ancestry analysis. For analyses of animals genotyped on different genotyping platforms, the marker list representing the intersection of the platforms can be provided to extract the markers that are common to all assays. The pipeline allows multiple input genotype files and uses the PLINK merge genotype files tool (--merge) to combine genotypes into a single file for downstream analysis.

\section{EIGENSOFT}

The EIGENSOFT convertf package is used to convert all genotypes from PLINK PED format into EIGENSTRAT format which is required by the SNPweights software. To process the reference panel data, principal component analysis 
(PCA) using EIGENSOFT smartpca is used to generate the eigenvalues and eigenvectors that are required to calculate SNP weights using SNPweights. However, the smartpca package included in EIGENSOFT versions beyond 5.0.2 is not compatible with SNPweights. SNPweights requires an input variable, "trace", to be located in the log file output from the smartpca analysis. For versions of EIGENSOFT beyond 5.0.2, the source code can be edited to ensure that the log file output is compatible with the SNPweights software (See Supplementary Information).

\section{SNPweights}

SNPweights implements an ancestry inference model based on genome-wide SNP weights computed using genotype data for an external panel of reference individuals. To obtain SNP weights, the matrix $\left(\mathbf{g}_{\mathrm{ij}}\right)$ of reference panel genotypes for SNP $\mathbf{i}=\mathbf{1}, \ldots, \mathbf{M}$ and individual $\mathbf{j}=\mathbf{1}, \ldots, \mathbf{N}$ is normalized by subtracting the

mean $\mu_{\mathrm{i}}=\mathrm{N}^{-1} \sum_{\mathrm{j}} \mathbf{g}_{\mathrm{ij}}$ and dividing by the standard deviation $\left[\mathrm{p}_{\mathrm{i}}\left(\mathbf{1}-\mathrm{p}_{\mathrm{i}}\right)\right]^{0.5}$ for each SNP, where $\boldsymbol{p}_{\mathbf{i}}=\boldsymbol{\mu}_{\mathbf{i}} / \mathbf{2}$, to improve the results of the subsequent PCA analysis from which a kinship matrix is generated [15]. A principal component decomposition is then used to generate the eigenvalues and corresponding eigenvectors of the kinship matrix [67]. The SNP weights file only needs to be recalculated if the reference panel is changed. EIGENSTRAT formatted target animal genotypes are input into SNPweights, along with the precomputed reference panel SNP weights. The SNP weights are then applied to the target individuals to estimate their ancestry proportions [67]. 


\section{Reference Panel Development}

The definition of a set of reference individuals that define the genotype frequencies at each SNP variant for each reference breed is technically demanding, but vitally important to the process of defining ancestry. This process assumes that selection has not operated to change gene frequencies between target and reference population animals, and that each population is sufficiently large that drift has not impacted allele frequencies. It also assumes that migration between different countries does not influence population allele frequencies when registered animals are imported or exported.

FastSTRUCTURE [65] analysis and iterations of animal filtering using SNPweights were performed using the genotypes of candidate reference panel individuals to remove individuals with significant evidence of admixture from the reference breed panel. An overview of the processes and iterations of filtering conducted in the development of this reference panel set is shown in Figure S2.1 and Table 2.1.

FastSTRUCTURE Analysis to Identify Candidate Reference Panel Individuals Genotype data for 48,776 individuals produced by one of 8 different genotyping assays were available for fastSTRUCTURE analysis (Table 2.1) [65]. We initially performed focused fastSTRUCTURE analyses using small numbers of reference breeds including Angus and Simmental, Angus and Gelbvieh, Angus and Limousin, Angus and Red Angus, Red Angus, Hereford, Shorthorn and Salers, Red Angus, Hereford and Shorthorn, and N'Dama, Nelore and Brahman (Figures 
S2.2-S2.8). Individuals possessing an ancestry assignment of at least $97 \%$ to their designated breed were retained for subsequent analysis (see Supplementary Methods and Table 2.1). Following filtering based on fastSTRUCTURE breed assignment, 17,852 individuals representing 19 of the original breeds remained for further analysis (Supplementary Methods and Figures S2.2-S2.8). All of the Salers animals were removed in this filtering analysis which is consistent with previous work that found that Salers and Limousin were very similar [4]. Variation in reference population sample sizes has been shown to substantially influence the estimation of the number of ancestral populations $(K)$ in ancestry analyses $[76,81,82]$. To minimize this effect and produce similar sample sizes for each of the reference breeds, we randomly sampled 200 individuals from each reference breed for which at least 200 individuals remained after filtering on an ancestry assignment of at least 97\%, otherwise all remaining individuals were included for the breed (Table 2.1). Following fastSTRUCTURE analysis using $\mathrm{K}=19$ after removal of Salers and using the BC7K marker set, Texas Longhorn was also removed from the reference panel breed list due to the inability to distinguish Texas Longhorn as a distinct population (Figure 2.2). Further, due to the known common ancestry [83] and similarity between Nelore and Brahman (Figure 2.2), the breeds were combined to represent B. t. indicus. 
SNPweights Analyses to Refine and Validate Reference Panel Members

Random sampling of reference breed individuals was performed to create sample sets containing $\leq n$ individuals per breed, for $n=50,100,150$ and 200 individuals (Figure 2.3 and Figures S2.9-S2.10). Sampling was performed such that if a reference breed had $\geq n$ candidates then $\mathrm{n}$ individuals were randomly sampled, otherwise, all available individuals were sampled. An analysis was performed using the BC7K marker set, SNPweights was used to assign reference breed ancestries to the same sample of individuals that was used to produce the SNP weights for each of the four samples of individuals (Figure 2.3 and Figures S2.9-S2.10). In the self-assignment analyses conducted using the reference breed sample sets of $\leq 100$ individuals per breed and $\leq 50$ individuals per breed, 7 individuals were removed due to their estimated breed ancestry being $\leq 60 \%$ to their registry breed (Holstein $n=3$, Jersey $n=1$, Japanese Black $\mathrm{n}=3$ ) (Figure 2.3).

\section{Breeds with Open Herdbooks}

For the Gelbvieh, Limousin, Shorthorn, Simmental, and Braunvieh breeds that have open U.S. herdbook registries, fullblood or $100 \%$ ancestry individuals were identified based on pedigree data obtained from the respective breed associations (Table 2.1). The term "fullblood" is used to identify cattle for which every ancestor is registered in the herdbook and can be traced back to the breed founders. The term "purebred" refers to animals that have been graded up via crossbreeding to purebred status. Charolais also has an open herdbook registry 
in the U.S., however, access to Full French imported Charolais breed members was limited. As a result, all Charolais individuals identified as purebred in the association registry were retained for downstream analysis, however, these individuals could contain up to $1 / 32^{\text {nd }}$ introgression from another breed. A random sample of 200 individuals was taken for each breed with more than 200 identified fullblood individuals, otherwise all animals were sampled. Individuals previously included in the candidate reference panel following preliminary fastSTRUCTURE filtering for the open herdbook breeds were removed and replaced with the fullblood individuals.

\section{Additional Reference Panel Filtering Using SNPweights}

After filtering animals identified to not be fullblood based on their pedigree information, we randomly sampled $\leq 50$ individuals per reference breed and utilized SNPweights to estimate weights for each sample and also to estimate breed ancestries for members of the same sample that was used to generate the SNP weights. Based on these analyses, we created 5 overlapping reference breed sets, each containing individuals with $\geq 90 \%, \geq 85 \%, \geq 80 \%, \geq 75 \%$, or $\geq 70 \%$ ancestry assignment to their registry breeds (Table 2.3).

\section{Simulated Genotypes}

Using the phased BC7K genotypes for the final reference population of 803 individuals ( 3 Nelore genotyped with the BovineHD assay were removed because they were determined to cause problems for the phasing software), we 
simulated genotypes for 803 individuals each generation $(\mathrm{N}=1,3,5$, and 10$)$ by randomly sampling two individuals as parents from generation $\mathrm{N}-1$ and using a Poisson distribution to sample, at random, a single recombinant chromosome from each parent. The number of recombination events for each sampled chromosome was sampled from a Poisson distribution with mean equal to chromosome length in Mb/100 (i.e. 1.58 Morgans for chromosome 1). Simulated genotypes were produced for individuals 1 generation removed from the fullblood/purebred reference population animals (i.e., $50 \%$ breed $A$ and $50 \%$ breed B), 3, 5, and 10, generations, respectively, to evaluate the ability of CRUMBLER to detect large through to small admixture proportions in animals with increasing numbers of breeds represented in their ancestry. Breed composition estimates for these animals were obtained by tracing the breed of origin of every allele present in each generation $\mathrm{N}$ animal. For each marker, we attributed the genomic fragment from the center points of the intervals on each side of each marker to the breed of origin of the two alleles at each marker and summed these across all loci. Finally, we normalized these sums by dividing by the autosomal genome size using UMD3.1 coordinates.

\section{Results and Discussion}

The concept of breed and breed membership is man-made and does not inherently exist in nature. Moreover, the formation of breeds of cattle is very recent, as cattle domestication began about 10,000 years ago but the formation of herdbooks has occurred only during the last 200-250 years [38]. Nevertheless, 
the effects of drift and human selection over the last 200 years have caused sufficient divergence among breeds that breed differences are identifiable at the molecular level. Such signals are essential for breed ancestry analyses to be effective in modern admixed animals. Previous work on assigning breed composition in admixed cattle utilized $50 \mathrm{~K}$ genotype data and a reference panel of 16 breeds, with the basis for reference panel inclusion being breed association registration [84]. However, the continual evolution of genotyping assays has led to content changes resulting in only a relatively small proportion of markers being in common among assays. Consequently, there is a need to evaluate whether these markers are sufficient for breed content estimation, leading to their conservation in the design of future assays. Furthermore the development of an analytical pipeline based on these markers would simplify analysis for end-users and the use of a single reference panel would allow the direct comparison of results between applications.

\section{Reference Panel Development}

Previously developed cattle reference panels have relied on pedigree accuracy and breed association registration for their definition [84]. Conversely, we used an iterative approach for reference population curation that was able to validate the accuracy of the pedigree information used to identify candidates. FastSTRUCTURE analyses performed using the candidate individuals for each of the initial 19 reference breeds suggested population subdivision in both the Hereford and Simmental (Figure 2.2). Pedigree analysis for the Herefords within 
each subpopulation indicated that the subpopulations comprised animals from the highly inbred USDA Miles City Line 1 Hereford population (L1) and other individuals representing broader U.S. Hereford pedigrees. The Miles City L1 Hereford cattle were derived from two bulls, both sired by Advance Domino 13 (AHA registration number 1668403) and 50 Hereford foundation cows. Since the founding of the L1 Herefords, the migration of germplasm has been unidirectional from L1 into the broader U.S. industry, as the L1 population has been closed since its founding [85]. However, the L1 Herefords have profoundly influenced the U.S. Hereford population. L1 Herefords do not segregate for recessive dwarfism, which has been a threat to Hereford breeders since the 1950s, and this has led to L1 cattle becoming popular in the process of purging herds of the defect [86]. In 1980, the average proportion of U.S. registered Herefords influenced by L1 genetics was 23\%. By 2008, this proportion had increased to $81 \%[85]$.

The detected subpopulation division within the Simmental breed (Figure 2.2) represents the differentiation between purebred and fullblood animals. For example, progeny of a popular fullblood Simmental sire are present in both subpopulations, however, in one subpopulation the family members are all fullblood and in the other they are all purebred or percentage Simmental animals. This result supports the need to identify fullblood animals as reference panel breed representatives for breeds with open herdbooks. 


\section{$\underline{\text { Reference population sample size }}$}

By randomly sampling individuals from the candidate reference breed set and using SNPweights to assign these individuals to reference populations, we found that reference panel breed sample sizes of $\leq 50$ or $\leq 100$ individuals appeared to capture the diversity within each breed and appropriately determined the ancestry of the tested individuals (Figure 2.3). For each breed, the percent ancestry predicted for the tested reference samples was, on average, $3.86 \%$ higher when the SNP weights were estimated using $\leq 50$ individuals per breed than when $\leq 100$ individuals per breed were used (Table 2.4). This reflects the increased homogeneity of individuals within each breed and a greater genetic distance between individuals from different breeds as smaller samples of individuals from each breed are used to define the reference panel. Further, due to limitations in the number of genotyped individuals for some breeds (Table 2.1), as the sample size was increased globally, imbalances were created between the reference panel breed sample sizes which impacted breed composition estimation (Figures S2.9-S2.10). It has previously been shown that the power to detect population structure improves as the reference population sample sizes become more similar $[76,82]$.

\section{Marker density}

After the replacement of reference breed individuals with those identified to be fullblood based on pedigree analysis for the open herdbook Gelbvieh, Simmental, Limousin, Braunvieh, Shorthorn, and Charolais breeds, additional 
self-assignment analyses were conducted to evaluate the effects of marker set size on ancestry prediction. Breed reference panels were again constructed by randomly sampling $\leq 50$ individuals per breed and SNP weights were calculated using both the $\mathrm{BC} 13 \mathrm{~K}$ markers and $\mathrm{BC} 7 \mathrm{~K}$ markers. The estimated SNP weights were then used to self-assign ancestry to members of the reference panel animals representing the reference breed set. The ancestry predictions for the reference breed individuals using either the BC7K (Figure 2.4a and Figure S2.11) or BC13K (Figure 2.4b and Figure S2.12) marker sets indicate that use of the BC13K marker set did not significantly impact the ancestry predictions.

Consequently, the use of the 6,799 markers common to the 8 commercially available genotyping platforms appears to be sufficient to assign breed ancestry for the majority of animals produced in the U.S. The CRUMBLER pipeline can accommodate samples genotyped using alternative assays, however, the produced breed composition estimates will be based on the intersection of markers on the assay and the $\mathrm{BC} 7 \mathrm{~K}$ marker set.

\section{Assignment thresholds}

We next examined the effects of reference breed homogeneity on ancestry assignment by identifying reference panel members that had been assigned to their breed of registry using SNPweights with probabilities of ancestry of $\geq 90 \%$, $\geq 85 \%, \geq 80 \%, \geq 75 \%$, and $\geq 70 \%$, respectively (Table 2.3 ). From these individuals, reference breed panels were obtained by randomly sampling $\leq 50$ individuals per breed, until each individual was represented in at least one sample set. SNP 
weights were then estimated using the $\mathrm{BC} 7 \mathrm{~K}$ marker set and ancestry was assigned for these individuals using SNPweights (Figures 2.5-2.6 and Figures S2.13-S2.15). Limiting the reference breed panel members to those individuals with $\geq 90 \%$ ancestry assigned to their breed of registry produced a reference panel that did not represent the extent of diversity within each of the breeds (Figure 2.5). On the other hand, using an ancestry assignment of $\geq 85 \%$ clearly captured greater diversity within each breed (Figure 2.6) and maximized the selfassignment of ancestry to the breed of registration (Table 2.5).

\section{$\underline{\text { Reference panel definition }}$}

To examine whether the specific individuals represented in the reference panel sample influenced the self-assignment of ancestry to the sampled individuals, a second sample of $\leq 50$ distinct individuals per breed was obtained from the individuals with $\geq 85 \%$ assignment to their breed of registration and analyzed with SNPweights (Figure 2.7). Figure 2.7 indicates that the ability to predict ancestry was not influenced by the specific individuals sampled from the set of animals with $\geq 85 \%$ ancestry to their breed of registration.

Additionally, Figures 2.6 and 2.7 suggest that the use of a reference breed panel constructed by the random sampling of $\leq 50$ individuals per breed from individuals with $\geq 85 \%$ self-assigned ancestry to their breed of registration maintained sufficient within-breed diversity to accurately estimate the ancestry of target individuals. However, these figures also reveal small amounts of apparent 
introgression from other reference panel breeds within each of the breeds. This does not appear to be an issue of marker resolution since the analyses performed with the $\mathrm{BC} 7 \mathrm{~K}$ and $\mathrm{BC} 13 \mathrm{~K}$ marker sets generated similar results (Figure 2.4). We conclude that these apparent introgressions are either due to a lack of power to discriminate among breeds using the common markers designed onto commercial genotyping platforms, or represent the presence of common ancestry among the breeds prior to the formation of breed herdbooks $\sim 200$ years ago. Molecular evidence for this shared ancestry exists, for example, Hereford and Angus cattle share the Celtic polled allele [87] and the segmental duplication responsible for the white anterior, ventral and dorsal coat color pattern occurs only in Hereford and Simmental cattle and their crosses [88]. These data clearly indicate that crossbreeding was widespread prior to the formal conceptualization of breeds.

\section{Reference Panel Validation}

To evaluate the ability of the selected reference breed panel to identify breed composition, an analysis was conducted for all 170,544 samples in the database which required 60 processor minutes (Figures 2.6-2.7). We extracted animals with pedigree information including fullblood and purebred animals registered with open herdbook breed associations and 2,243 crossbred animals with varying degrees of admixture. Considering the amount of available data, the number of pedigreed admixed animals was very limited and the purebred animals all had similar expected admixture proportions. Consequently, we next 
simulated genotypes for animals by assuming the random mating of members of the reference breed panel for $1,3,5$ and 10 generations assuming nonoverlapping generations to generate generations of animals with different numbers of breeds and breed proportions represented in their genomes.

\section{$\underline{\text { Registered fullblood animals }}$}

For the Gelbvieh, Limousin, Shorthorn, Simmental, and Braunvieh breeds that have open herdbook registries, fullblood or $100 \%$ ancestry individuals were identified based on pedigree data obtained from the respective breed associations (Table 2.1). CRUMBLER estimates were obtained for these fullblood individuals and the distribution of estimates by breed are in Figure 2.8. For all breeds except Charolais, $>50 \%$ of the individuals had CRUMBLER estimated percentages of $\geq 80 \%$ to their respective breeds. Average percentage estimates for fullblood Gelbvieh, Limousin, Shorthorn, Simmental, and Braunvieh individuals were $76 \%, 78 \%, 83 \%, 79 \%$, and $85 \%$, respectively (Figure $2.8 \mathrm{~b}$ ). However, the number of genotyped imported Full French Charolais animals was limited and so we also analyzed all purebred Charolais individuals which could contain up to $1 / 32^{\text {nd }}$ of their genome introgressed from another breed. The average Charolais breed assignment was $72 \%$ and the distribution of estimates was more variable than for the fullblood animals from the other breeds (Figure 2.8b). 


\section{$\underline{\text { Pedigreed crossbred animals }}$}

Based on pedigree, 2,005 individuals were identified as being primarily Hereford but with varying degrees of Red Angus, Salers, Angus or unknown other breed influence. The analysis results agreed with the pedigree data (Figure 2.9a) To investigate the correlations between pedigree and CRUMBLER estimated breed proportions, we removed proportions for breeds that were less than $3 \%$ and normalized the remaining values. CRUMBLER estimates were then correlated with the pedigree predicted estimates of the proportion of Hereford in these individuals (Figure 2.9c). CRUMBLER tended to underestimate the Hereford proportion as the pedigree estimated Hereford proportion tended to $100 \%$.

The remaining 238 crossbred individuals were commercial, advanced generation animals with an expected $50 \%$ Angus and $50 \%$ Simmental ancestry based on pedigree data. Results of the CRUMBLER analysis again support the pedigree data (Figure 2.10). The presence of Red Angus ancestry in these animals reveals the inability of the analysis to fully differentiate between Angus and Red Angus, which only diverged in the U.S. in 1954, and also the influence of Red Angus in the U.S. Simmental breed (Figure S2.16).

\section{$\underline{\text { Simulated genotypes }}$}

Genomes were simulated using the phased genotypes for 803 individuals from the reference breed panel to contain varying breed numbers and admixture proportions after $1,3,5$, and 10 generations of random mating with 
nonoverlapping generations. In generation 1 , the admixed individuals were $F_{1}$ individuals with a 50:50 autosomal genome composition unless both parents were randomly sampled from the same breed. CRUMBLER estimates of breed composition using the simulated genotypes were strongly correlated with the simulated compositions, especially for generations 1 and 3 (Figure 2.11). As the number of generations increased, the number of breeds represented in the simulated genomes tended to increase and the proportion of the genome originating from any one breed tended to decrease and the correlation between the simulated proportions and CRUMBLER estimates also decreased. Nevertheless, by generation $10,44 \%$ of animals had their genome proportions estimated with a correlation of at least $70 \%$. In the U.S. commercial crossbreeding does not usually involve the use of more than 3-4 breeds of cattle and while the number of generations of crossbreeding may very well be 10 or perhaps more, many generations will involve the mating of animals with similar genome ancestries and the proportions for each breed will be much greater than present in the generation 10 animals in Figure 2.11. Consequently, the achieved accuracies are likely to be closer to the generation 3 or 5 results where $99 \%$ and $68 \%$ of animals, respectively, had their genome proportions estimated with a correlation of greater than $80 \%$.

\section{Advanced generation composite animals}

The ancestry model assumes that neither drift or selection has acted to alter the allele frequencies from those created by the initial admixture proportions. We 
examined CRUMBLER estimates of breed composition for advanced generation members of the Brangus $(n=11,362)$, Beefmaster $(n=3,832)$ and Santa Gertrudis $(n=2,010)$ composite breeds where selection has had the opportunity to change breed composition from expectations at breed formation. Brangus individuals are expected to be $5 / 8$ Angus and $3 / 8$ Brahman, Beefmaster individuals $1 / 4$ Hereford, $1 / 4$ Shorthorn, and $1 / 2$ Brahman, and Santa Gertrudis $5 / 8$ Shorthorn and $3 / 8$ Brahman, respectively. These breeds use mating strategies that produce individuals that are expected to possess these proportions for registration within each of the respective breed's herdbook. However, registerable animals are ultimately advanced generation composites and so drift, meiotic sampling of parental chromosomes and selection are all expected to create individual variation in these ancestry proportions. CRUMBLER results for these advanced generation composites, also known as the American breeds, are shown in Figure 2.12. Table 2.6 contains the average breed proportion estimates assigned to each of these breeds by CRUMBLER and their standard deviations across the animals analyzed for each breed. In every instance, CRUMBLER underestimates the expected proportions for each of the American breed populations, however, the ancestral breeds clearly dominate the assignments (Table 2.6). Interestingly, on average, CRUMBLER estimated proportions of Holstein ancestry for advanced generation Beefmaster and Brangus animals (Figure 2.12 and Table 2.6). These American breeds do not contain any Holstein introgression and they do not contain ancestry from a "Ghost Population", a population that is not present in the reference set, which would lead to a breed assignment to a reference breed that 
it most closely resembled [76]. We speculate that this effect is caused by selection creating a deviation in allele frequencies from those found in the founder breeds which the model explains by an introgression from a distantly related breed, in this case, Holstein. Stratifying these genotyped animals according to the number of generations from foundation fullblood animals and examining the extent of estimated Holstein introgression, which would be expected to increase with generation number, would enable this to be tested, but we did not have access to the necessary data. However, this hypothesis is supported by the fact that the Santa Gertrudis had the least estimated Holstein introgression and the breed has published estimates of additive genetic merit for many fewer years than the Beefmaster or Brangus.

\section{Admixture}

We also tested the ADMIXTURE software [22] for ancestry estimation and integration into the CRUMBLER pipeline using the same reference breed panel that was developed for use with SNPweights. ADMIXTURE uses maximum likelihood estimation to fit the same statistical model as STRUCTURE, however, STRUCTURE does not allow the specification of individuals of known descent to be used as a reference panel [27]. ADMIXTURE allows a supervised analysis, in which the user can specify a reference set of individuals, by specifying the "-supervised" flag and requires an additional file with a ".pop" suffix to specify the genotypes of the reference population individuals [27]. Unlike SNPweights, the reference population individuals' genotypes must be provided in a genotype file for each analysis. 
We first conducted an ADMIXTURE analysis in which we self-assigned ancestry for the animals in the reference breed set formed with $\leq 50$ individuals per breed from the individuals that had $\geq 85 \%$ assignment to the breed of registration (Figure 2.13). The results shown in Figure 2.13 are similar to those in Figure 2.6 for the same reference panel, albeit with perhaps less evidence of background introgression. We next conducted an analysis using the reference panel used in Figure 2.13 merged with data for the 2,005 high percentage crossbred Herefords animals. The results shown in Figure 2.14, reveal a significant change in the ancestry proportions estimated for the reference panel Guernsey, Gelbvieh and Romagnola individuals between the two analyses which used exactly the same reference panel, but differed only in the number of individuals for which ancestry was to be estimated. This suggests that ADMIXTURE may use the target individuals to update information provided by the reference panel individuals specified in the ".pop" file. Consequently, the ADMIXTURE estimated ancestry proportions appear to be context dependent and may vary based on the other individuals included in the analysis.

Moreover, the order in which the target individuals appear in the genotype input file also appears to affect ADMIXTURE estimates of ancestry proportions for the target individuals. Figure 2.15 shows the results of an ADMIXTURE analysis in which the target individuals were identical to those shown in Figure 2.14, but for which the order of the reference individuals and the 2,005 Hereford crossbred 
individuals was reversed in the input files. In Figure 2.14, the reference individuals appear before the 2,005 Hereford crossbred individuals in the input file, whereas in Figure 2.15, the 2,005 Hereford crossbred individuals appeared before the reference individuals in the input file. The results reveal a significant change in ancestry proportions for Guernsey and Gelbvieh, but the Romagnola now appear to be non-admixed. Finally, we performed an ADMIXTURE analysis for these animals in which the order of animals in the input genotype file was completely randomized (Figure 2.16). Following analysis, the individuals were sorted to generate Figure 2.16. Again, the ancestry proportions for the Guernsey, Gelbvieh and Romagnola individuals suggest these breeds to be admixed.

STRUCTURE and ADMIXTURE are widely used for characterizing admixed populations [76], however, we have not found any reports in the literature that indicate that the software is sensitive to the input order of individuals. However, we suspect that the majority of users would have no need, or motivation, to run the software with permuted data input files. Nevertheless, because of these inconsistencies between results, we chose to not use ADMIXTURE for ancestry estimation within the CRUMBLER pipeline.

\section{Broader Application Using Additional Commercially Available Assays}

To broaden the spectrum of data from different commercially available assays that can be evaluated, an additional intersection of markers was obtained using 11 commercially available bovine assays including the GGP-90KT, GGP-F250, 
GGP-HDV3, GGP-LDV3, GGP-LDV4, BovineHD, BovineSNP50, i50K, Irish Cattle Breeding Federation (Cork, Ireland) IDBv3, and GeneSeek (Lincoln, NE) BOVG50v1 assays. The intersection SNP set included 6,363 SNPs (BC6K). A SNPweights self-assignment analysis using the reference set of individuals with $\geq 85 \%$ assignment to their breed of registration was conducted to assess the effects of the reduction in number of markers used for ancestry assignment. The ancestry proportions assigned based on the BC6K marker set (Figure 2.17) did not differ appreciably from those obtained using the BC7K marker set (Figure 2.6). This result indicates the utility of CRUMBLER and the reference panel breed set across the spectrum of commercially available genotyping platforms.

\section{Conclusions}

The determination of a set of reference population breeds and individuals that define allele and genotype frequencies at each variant for each of the breeds is arguably the most important, yet technically difficult step in the process of ancestry estimation. We employed several iterations of filtering to remove recently admixed individuals and identify a relatively homogeneous set of individuals that nevertheless represented the variation that might be expected among individuals within a breed. Once determined, the reference panel genotype data need only be processed once to obtain SNP weights removing the need to share genotype data for reference individuals in subsequent studies [67]. The upfront development of an external reference breed panel capitalizes on the rich ancestry information available in large available datasets, and relatedness, 
variation in sample sizes and diversity among the target individuals does not affect the inference of ancestry [67].

In cattle, the visual evaluation of breed characteristics is a poor method for evaluating the ancestry of individuals. Breed association pedigrees can be used to estimate expected breed compositions, however, the random assortment of chromosomes into gametes and selection can lead to ancestry proportions that differ from those expected based upon pedigree. Moreover, the vast majority of commercial beef cattle in the U.S. have no or very limited pedigree information and since these animals are frequently used for genomic research [50-52], there is a need for a tool that can routinely provide ancestry estimates for downstream use in GWAA or other genetic studies.

We tested ADMIXTURE and SNPweights and found that results from ADMIXTURE appear to depend on the ancestry and order of appearance of individuals within the genotype input file. We therefore developed an analysis pipeline, CRUMBLER, based upon PLINK, EIGENSOFT and SNPweights to automate the process of ancestry estimation. The developed bovine pipeline utilizes the 6,799 SNPs present on 8 commercially utilized bovine SNP genotyping assays and results using these SNPs are consistent with results obtained when 13,291 SNPs were used. From an available 48,776 genotyped individuals, we also developed a reference panel of 806 individuals sampled from 17 breeds to have $\leq 50$ individuals per breed that had $\geq 85 \%$ assignment to their 
breed of registration. This panel appears to allow the robust estimation of the ancestry of advanced generation admixed animals, however, all breeds share some common ancestry which predates the recent development of breed association herdbooks [37, 38].

CRUMBLER is not limited to application in cattle and with the provision of suitable reference breed allele frequencies can be applied to other species for ancestry estimation. CRUMBLER pipeline scripts and reference panel breed SNP weights are available on GitHub (https://github.com/tamarcrum/CRUMBLER). This pipeline tool is released under the GNU General Public License. 


\section{Figures}

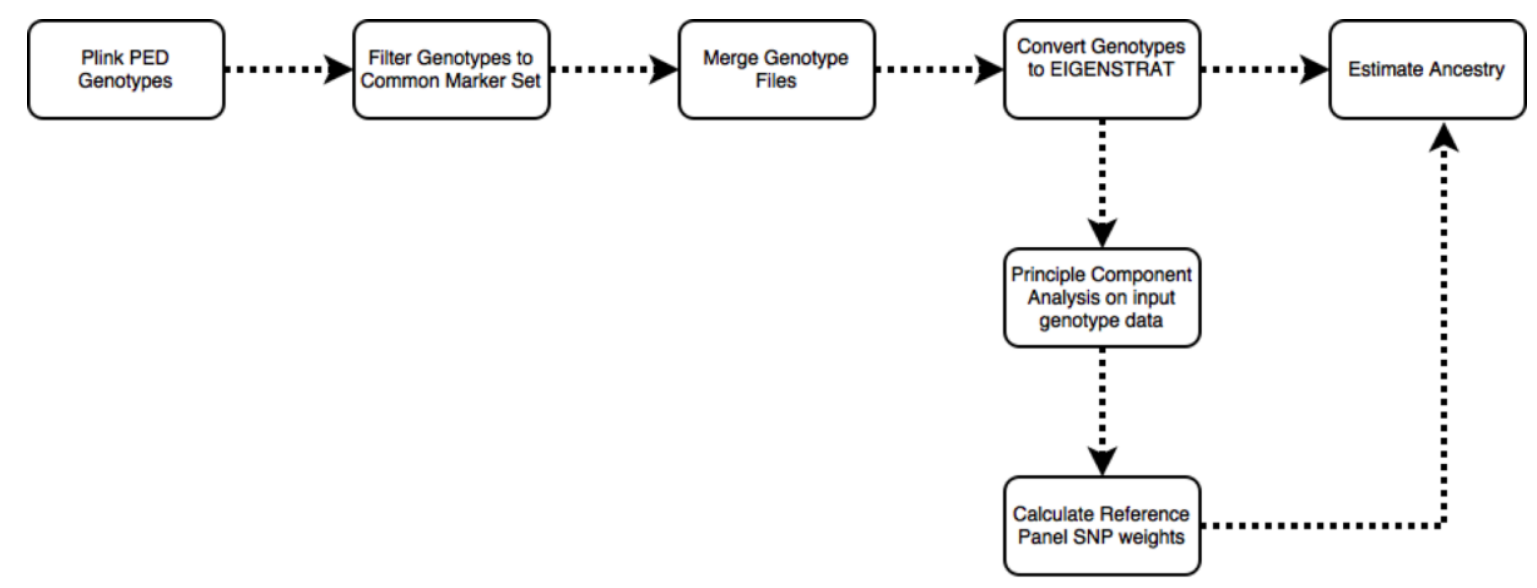

Figure 2.1. Flow diagram of the breed composition pipeline. 

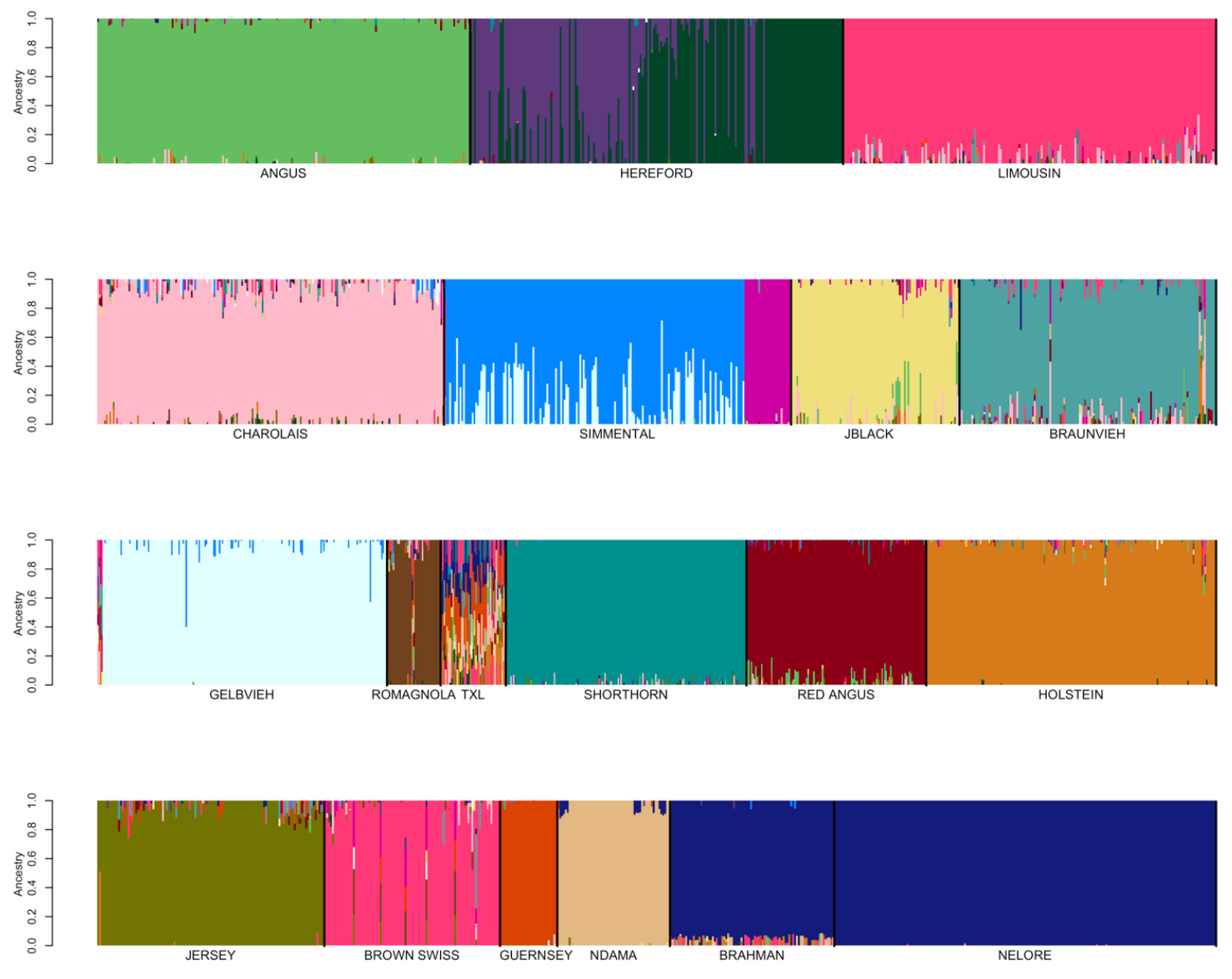

Figure 2.2. FastSTRUCTURE results for a random sample of $\leq \mathbf{2 0 0}$ individuals per breed from the pool of 17,852 potential reference individuals at $K=19$. Breed identification is shown below each colored block and each animal is represented as a vertical line within the block. 

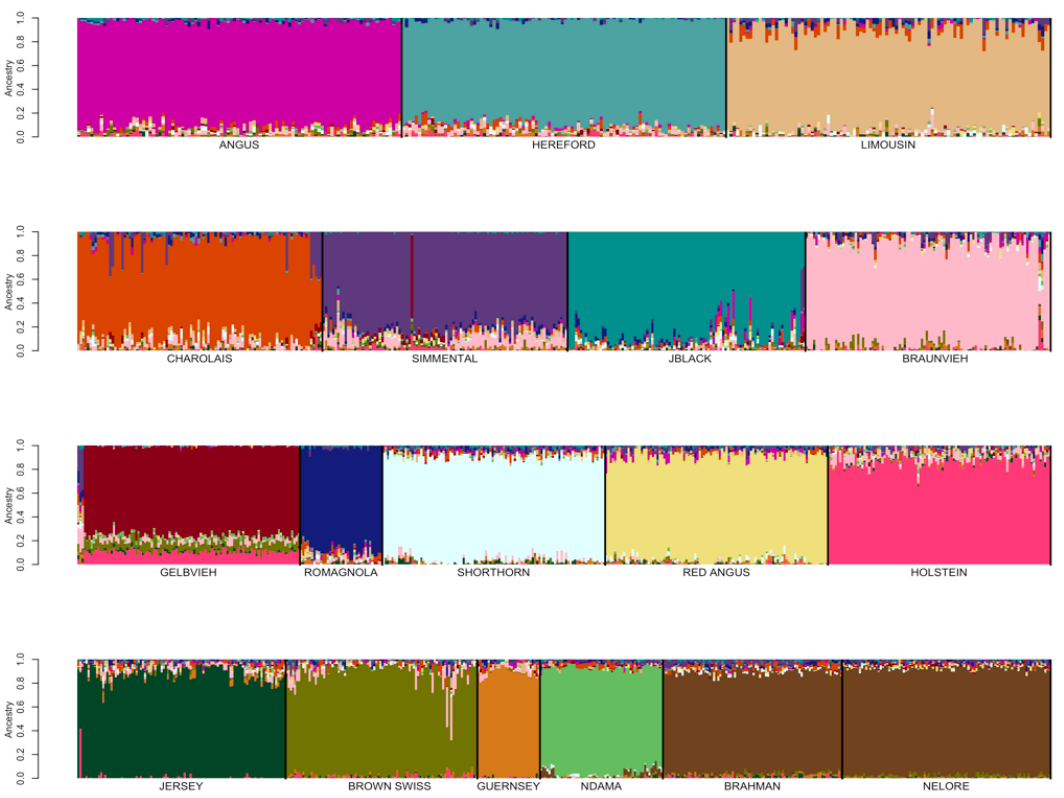

a.
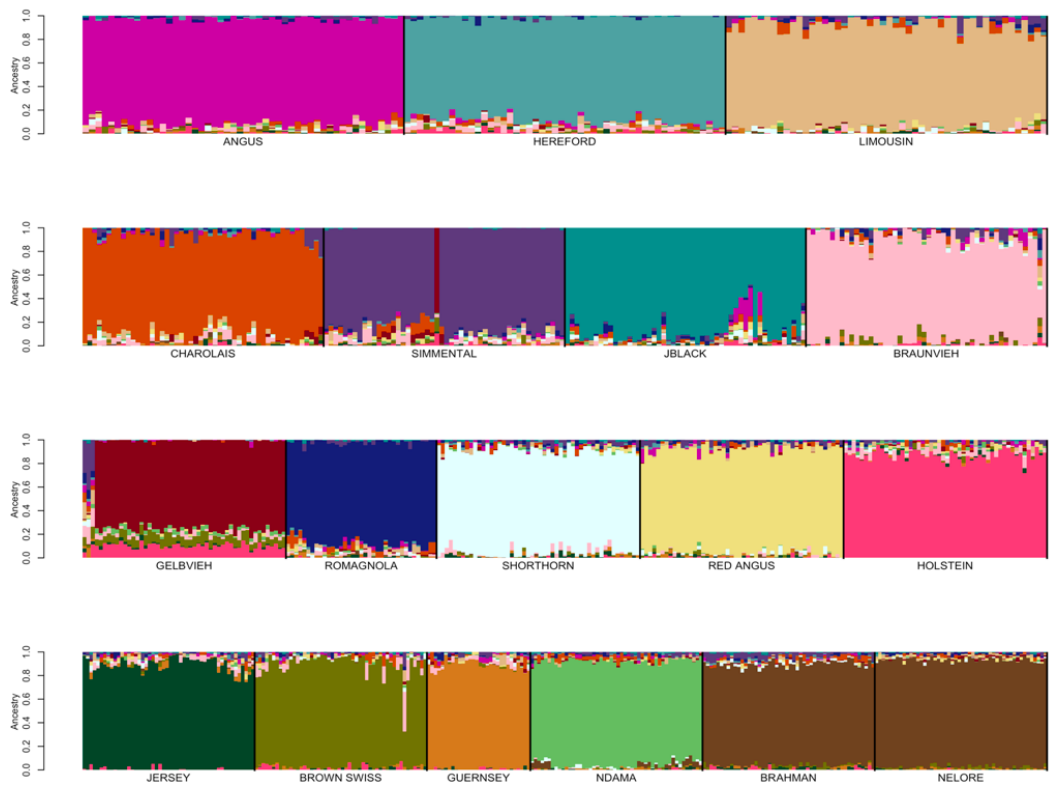

b.

Figure 2.3. SNPweights self-assignment analysis results for reference panel sample sets consisting of: (a) $\leq 100$ individuals per breed, or (b) $\leq 50$ individuals per breed. Seven individuals were filtered for $\leq 60 \%$ ancestry to their breed of registry (Holstein $n=3$, Jersey $n=1$, Japanese Black $n=3$ ). 

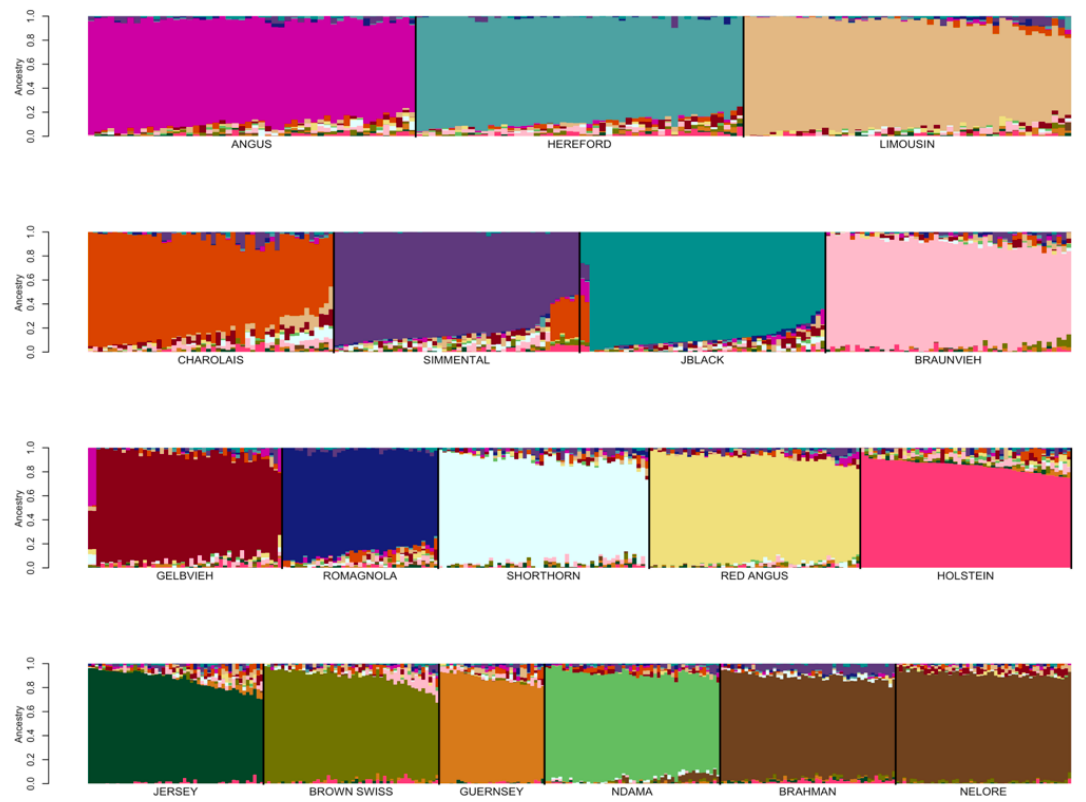

a.
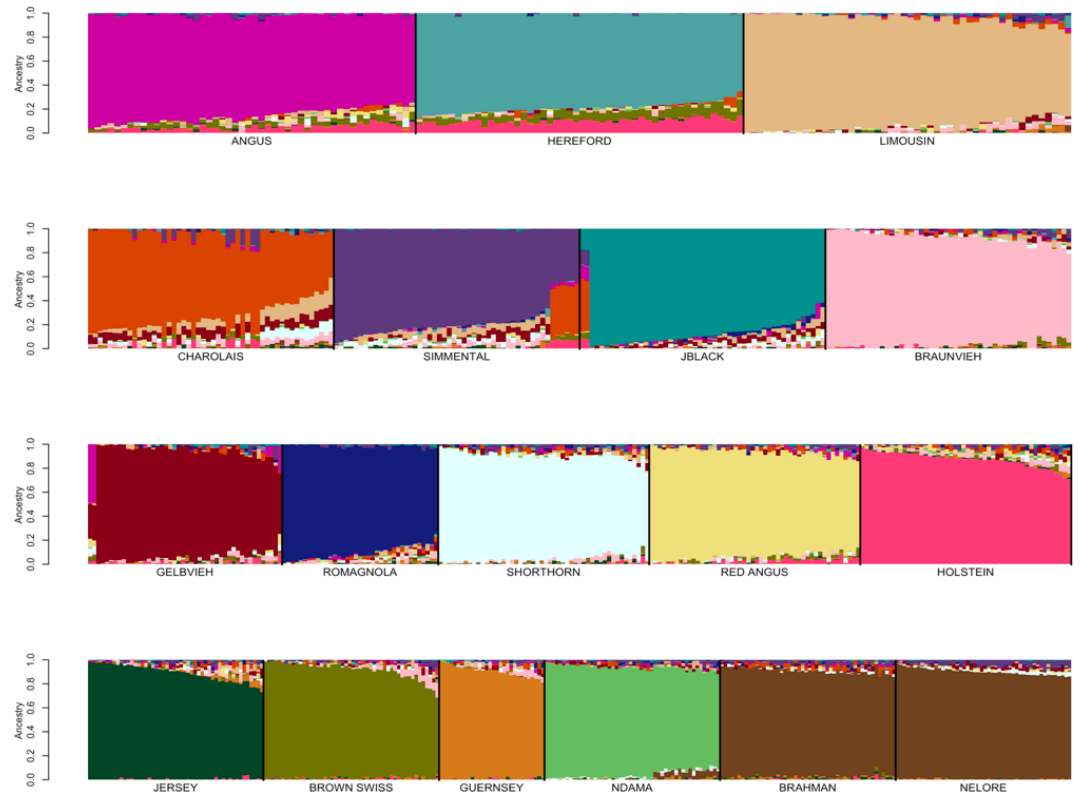

b.

Figure 2.4. SNPweights self-assignment of ancestry for candidate reference breed individuals following evaluation of open herdbook breeds using: (a) the $\mathrm{BC7K}$, or (b) the $\mathrm{BC13K}$ marker panels. Reference breed panels were constructed by random sampling $\leq 50$ individuals per breed and SNP weights were estimated using the $\mathrm{BC} 7 \mathrm{~K}$ and $\mathrm{BC} 13 \mathrm{~K}$ marker sets. 

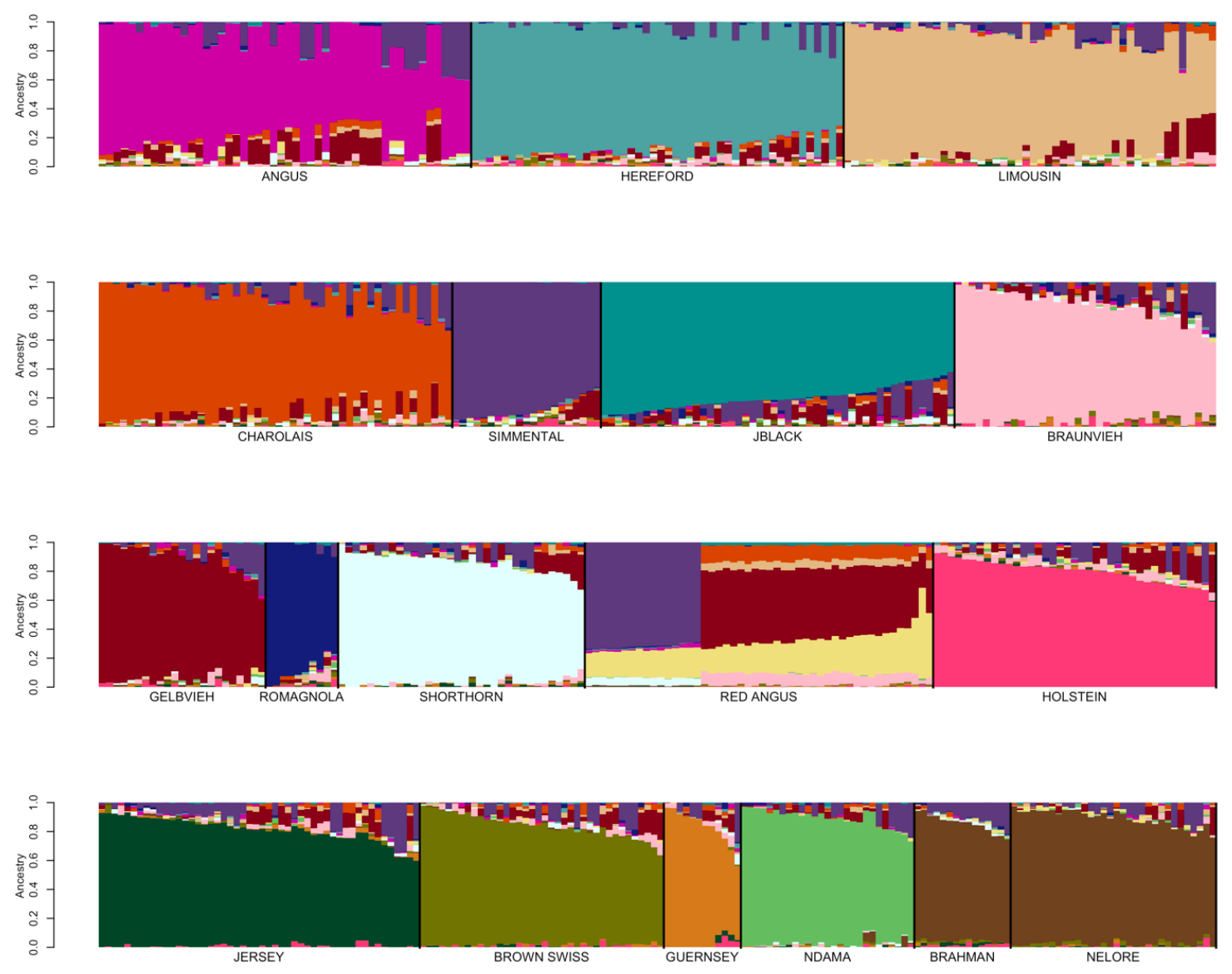

Figure 2.5. Reference breed panel constructed by the random sampling of $\leq 50$ individuals per breed from individuals with $\geq 90 \%$ ancestry was selfassigned to reference breed ancestry using the BC7K marker set. 

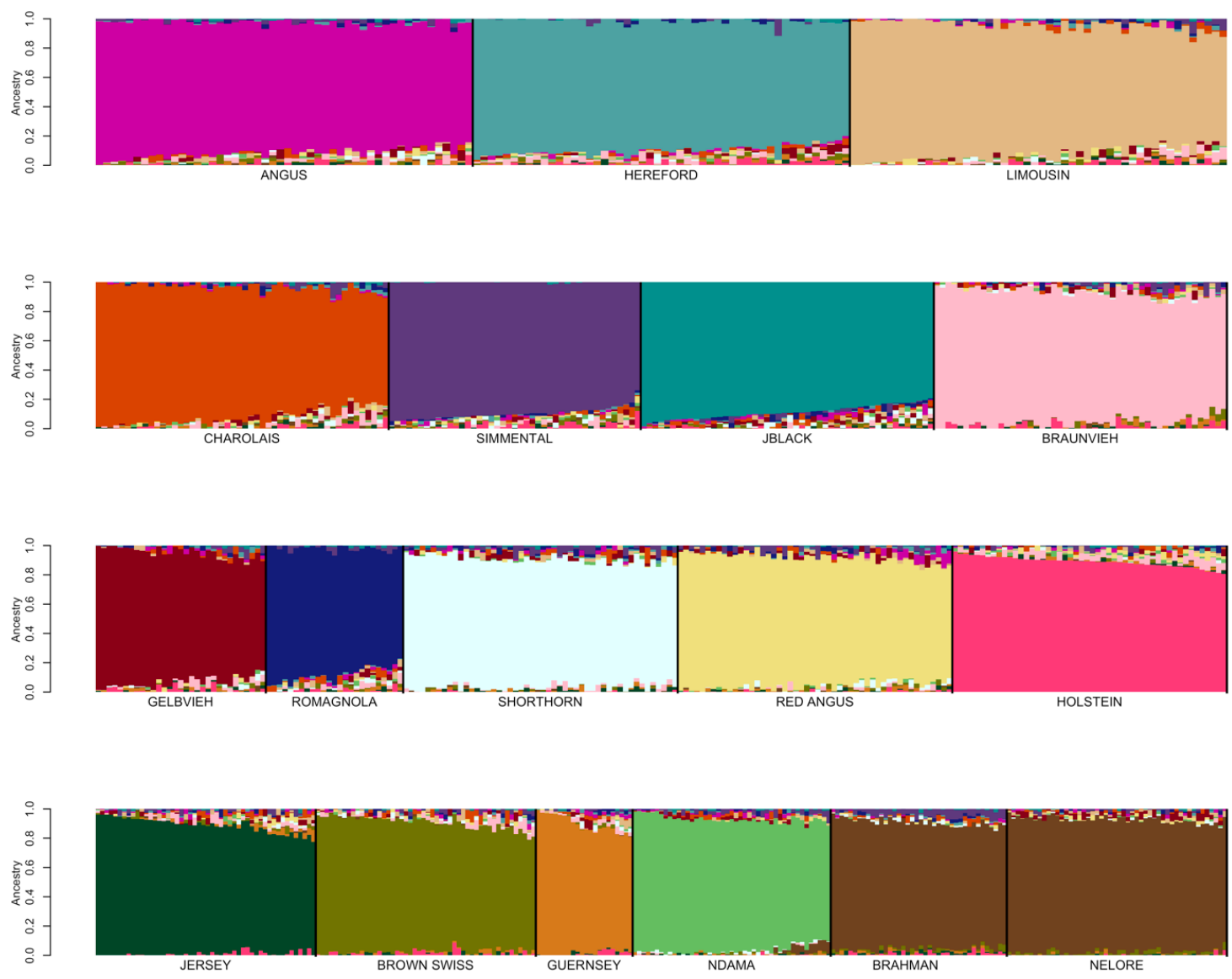

Figure 2.6. Reference breed panel constructed by the random sampling of $\leq 50$ individuals per breed from individuals with $\geq 85 \%$ ancestry was selfassigned to reference breed ancestry using the BC7K marker set. 

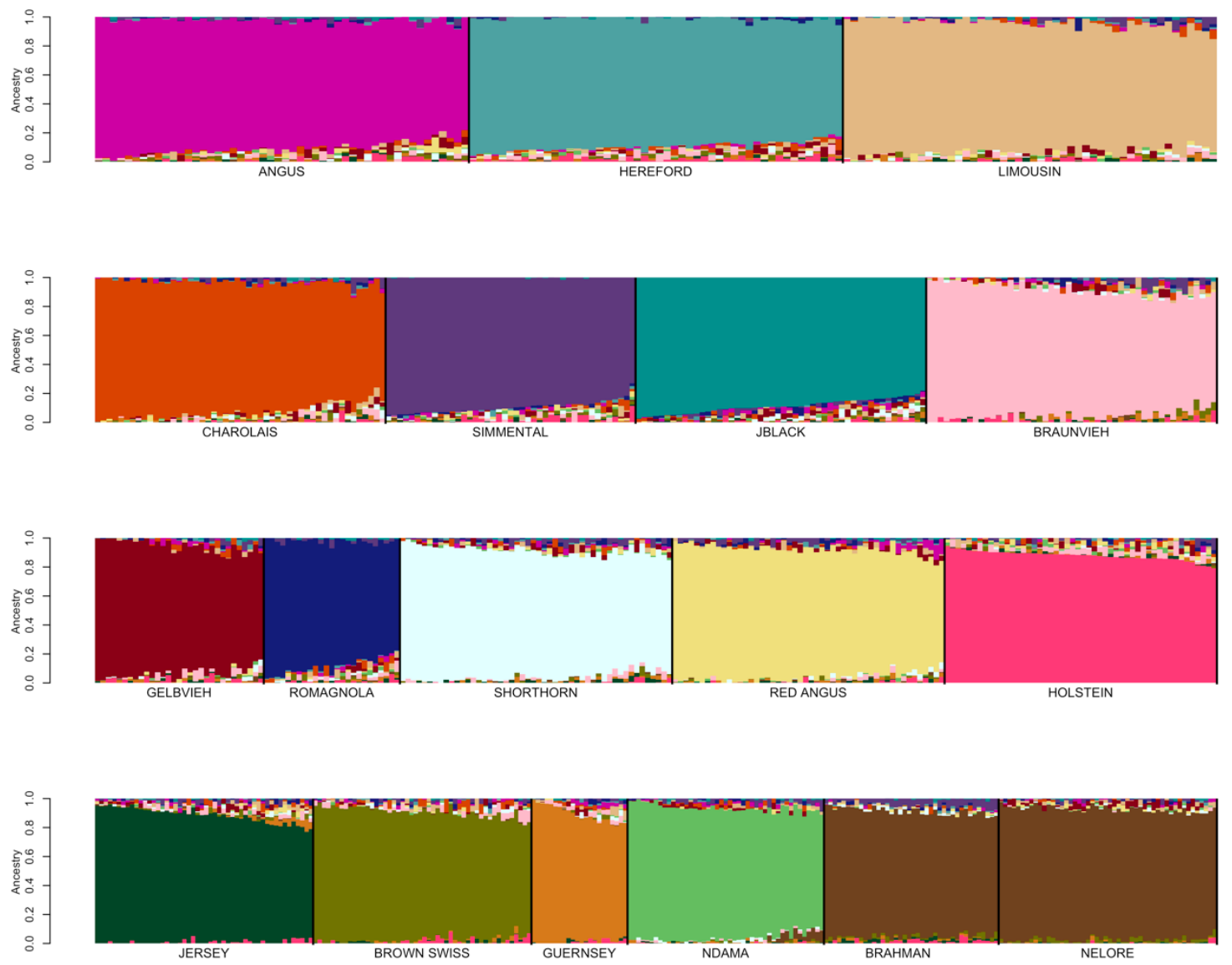

Figure 2.7. Reference breed panel constructed by the independent random sampling of a second sample of $\leq \mathbf{5 0}$ individuals per breed from individuals with $\geq 85 \%$ ancestry after eliminating individuals represented in the first sample was self-assigned to reference breed ancestry using the $\mathrm{BC}$ KK marker set. 


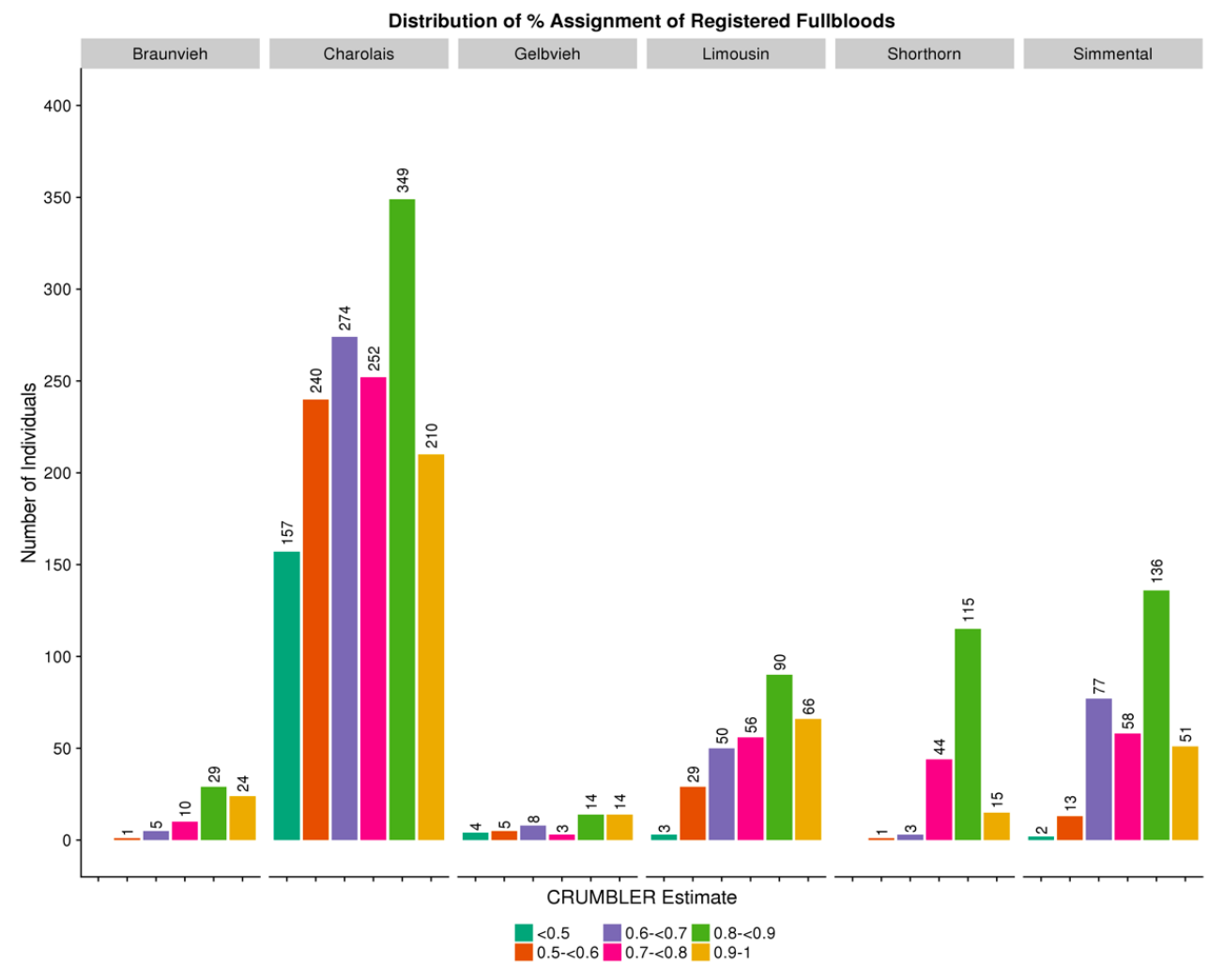

a.

Fullblood Cattle CRUMBLER Estimated Breed Composition

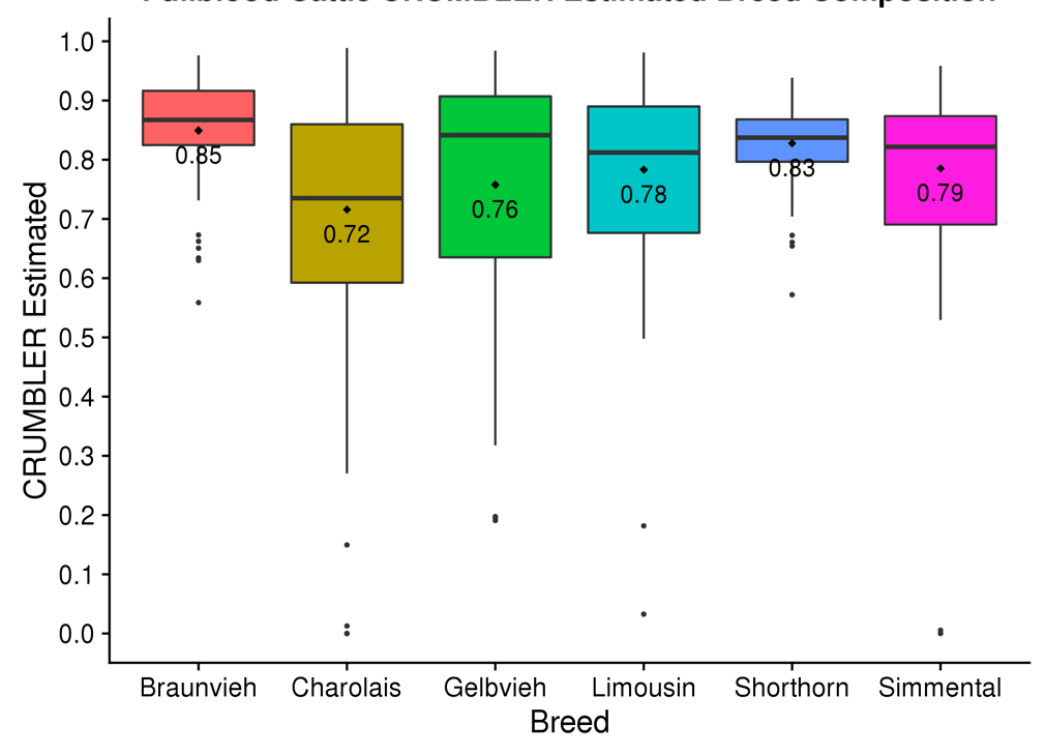

Breed

审Braunvieh

由harolais

审 Gelbvieh

由imousin

Shorthorn

Simmental

b.

Figure 2.8. (a) Distribution by breed of SNPweights ancestry assignment results for 2,408 registered fullblood animals from open herdbook breeds. (b) Pictorial representation of CRUMBLER estimates for 2,408 registered fullblood animals from open herdbook breeds. 


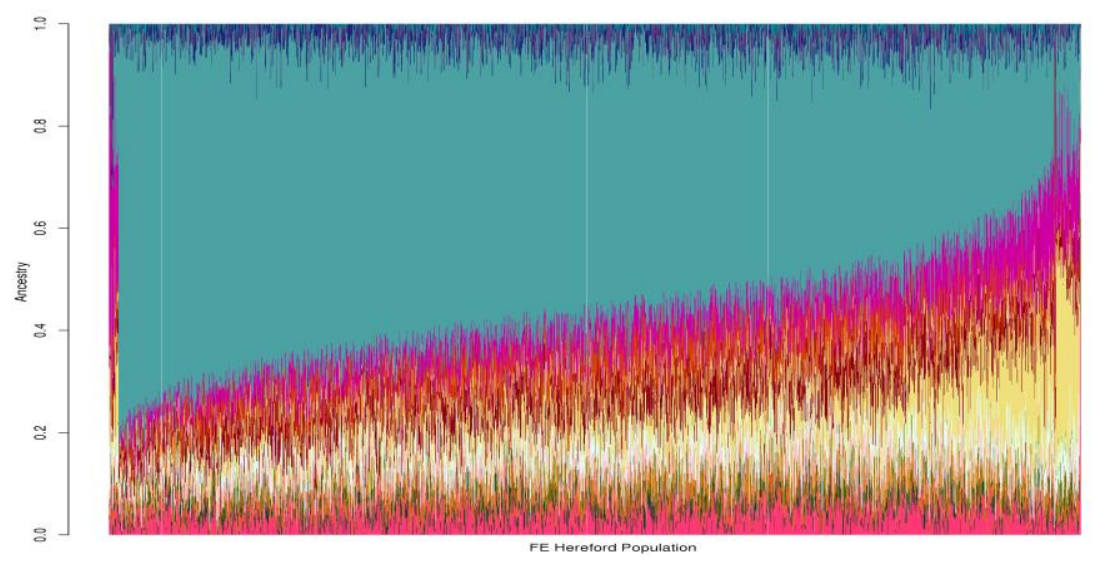

a.

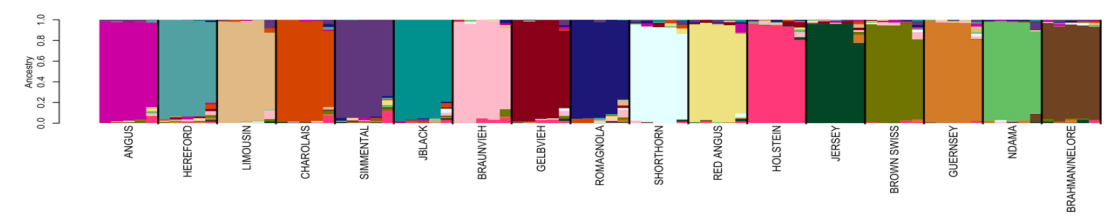

b.

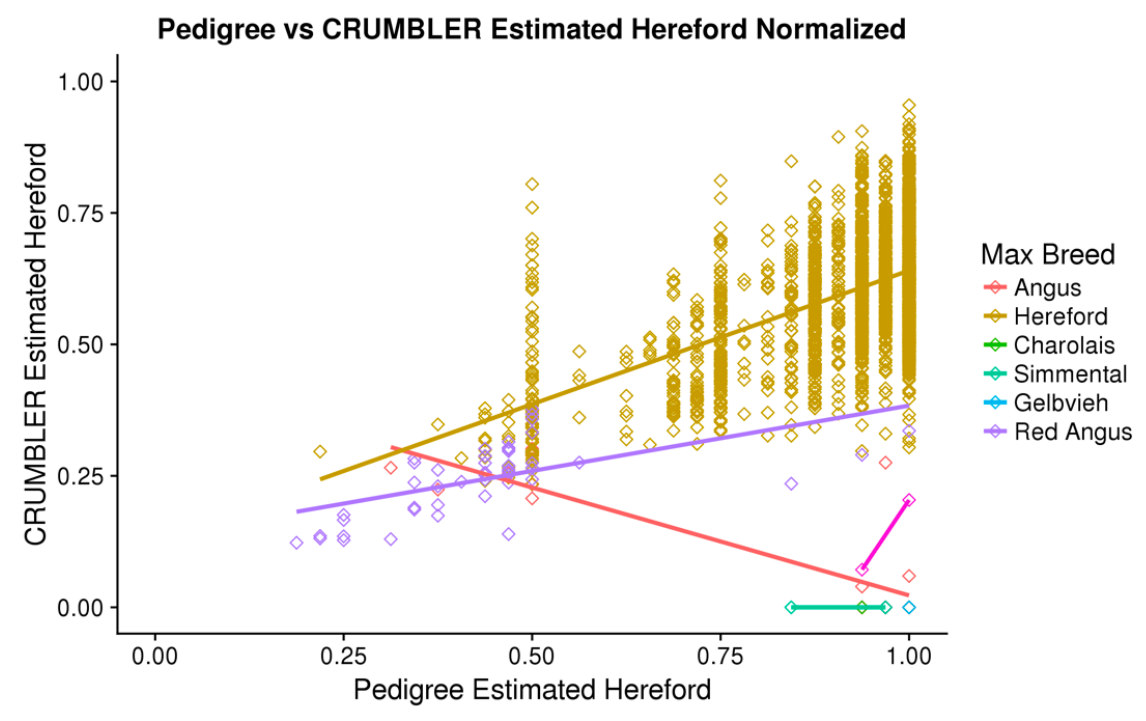

Figure 2.9. (a) SNPweights ancestry results for 2,005 crossbred Hereford individuals with a-priori breed composition estimates determined by pedigree. (b) Breed assignment reference breed key. (c) Hereford SNPweights estimated proportions using CRUMBLER are plotted against the pedigree estimates. Data point color indicates the breed for which SNPweights assigned the highest proportion for each individual. 


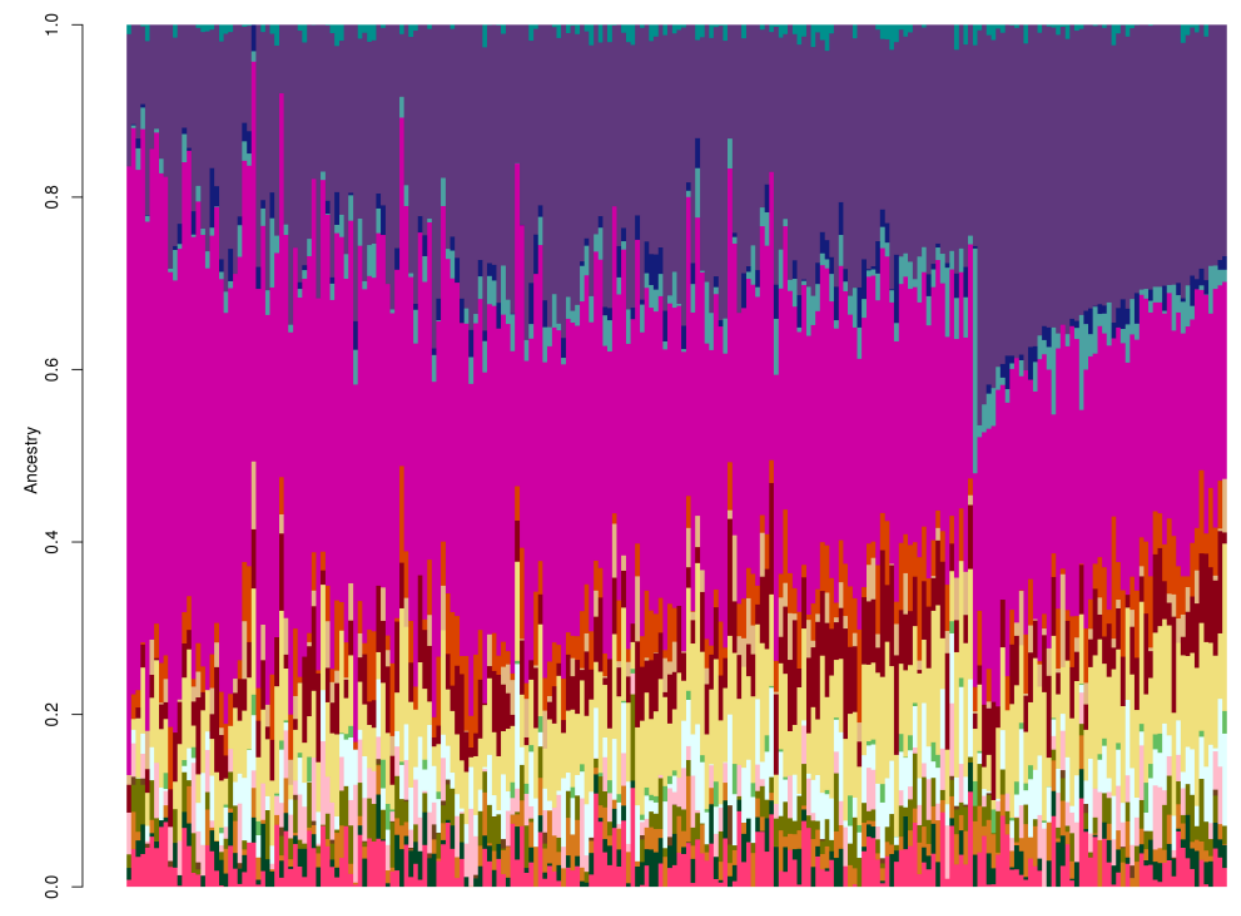

a.

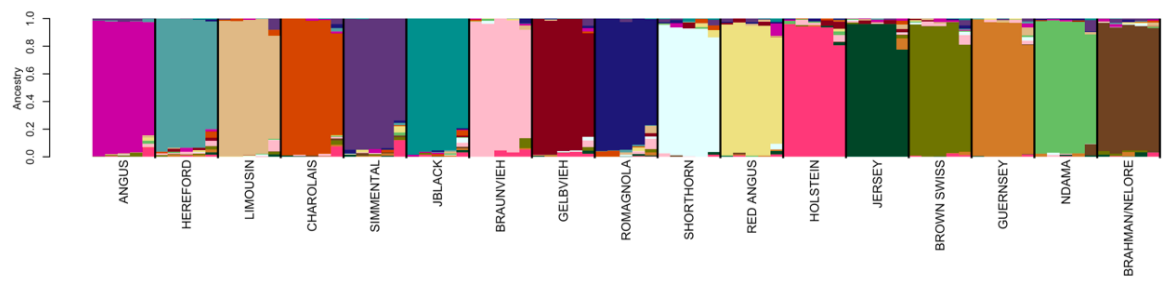

b.

Figure 2.10. (a) SNPweights ancestry results for 238 crossbred individuals with a-priori breed composition estimates of $50 \%$ Angus and $50 \%$ Simmental based on a reference panel with $\leq 50$ individuals per breed sampled from individuals with $\geq 85 \%$ assignment to their breed of registry. (b) Breed assignment for the crossbred individuals can be determined using this reference breed key. 
Correlations between CRUMBLER and Simulated Breed Proportions

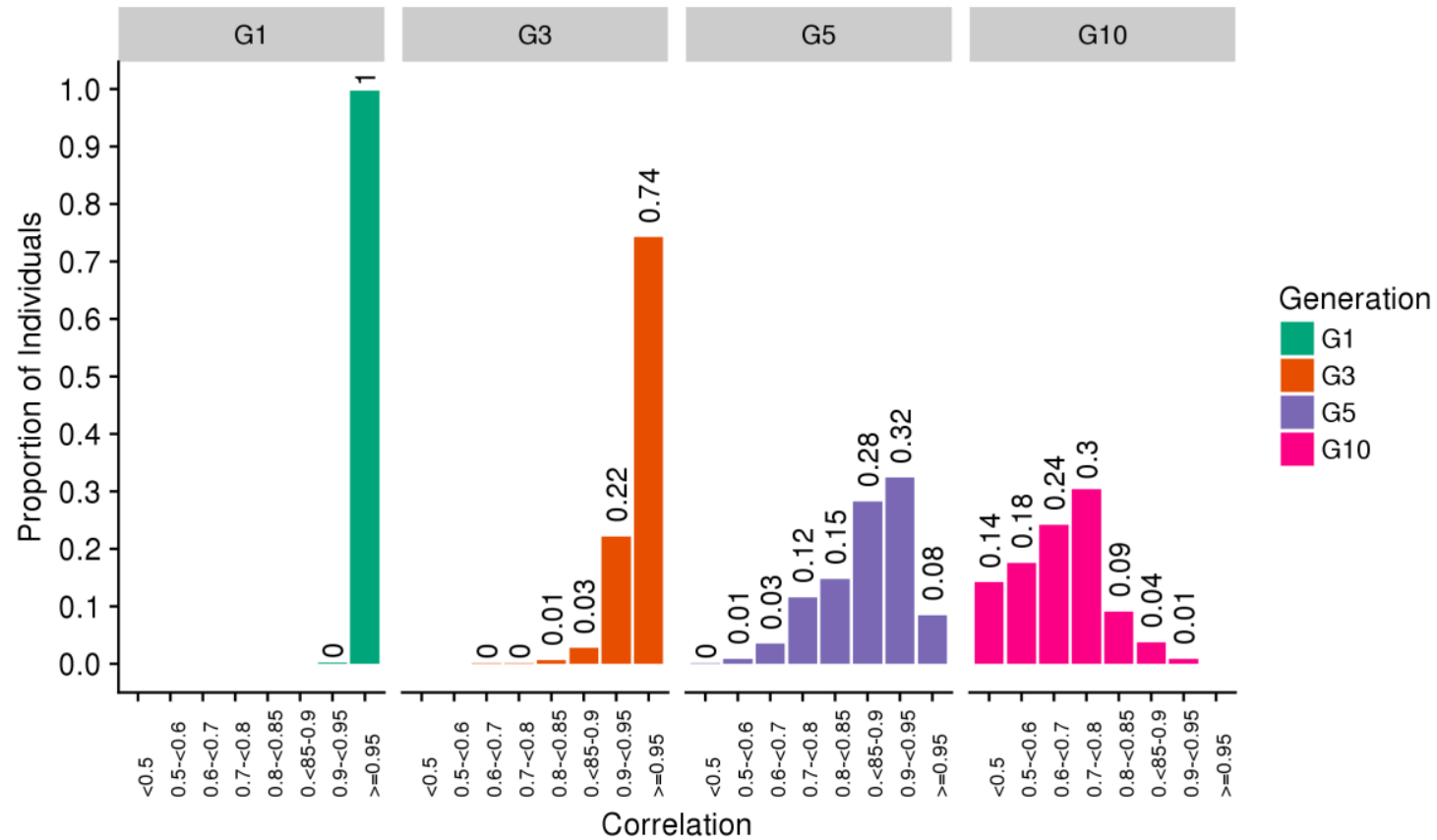

Figure 2.11. Genotypes were simulated for the indicated number of generations of random mating, with generation 1 (G1) animals being 50:50 proportion except when two parents from the same breed were mated. SNPweights results were obtained using CRUMBLER pipeline parameters correlations between these estimates and the known simulated breed compositions were produced and the proportion of individuals within each correlation class is indicated. 

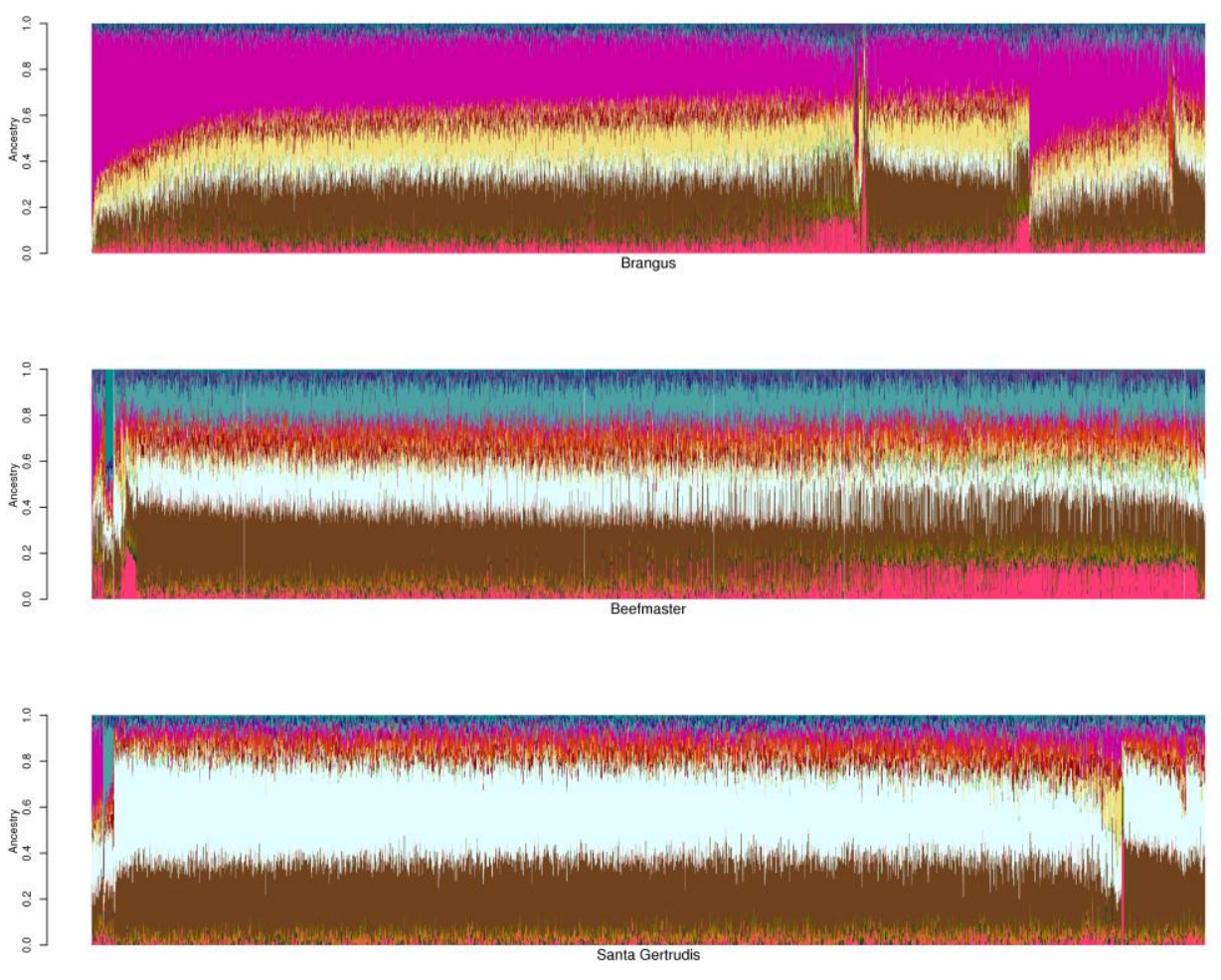

a.

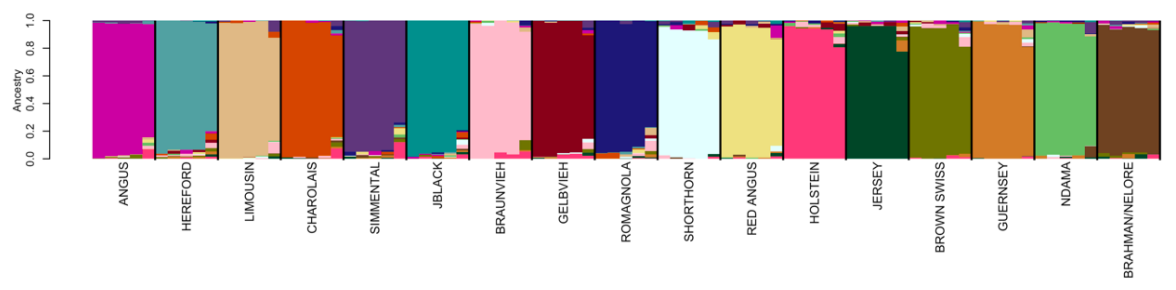

Figure 2.12. (a) SNPweights ancestry results using CRUMBLER pipeline for 11,362 Brangus, 3,832 Beefmaster, and 2,010 Santa Gertrudis individuals. (b) Breed assignment for these advanced generation composite animals can be determined using this reference breed key. 

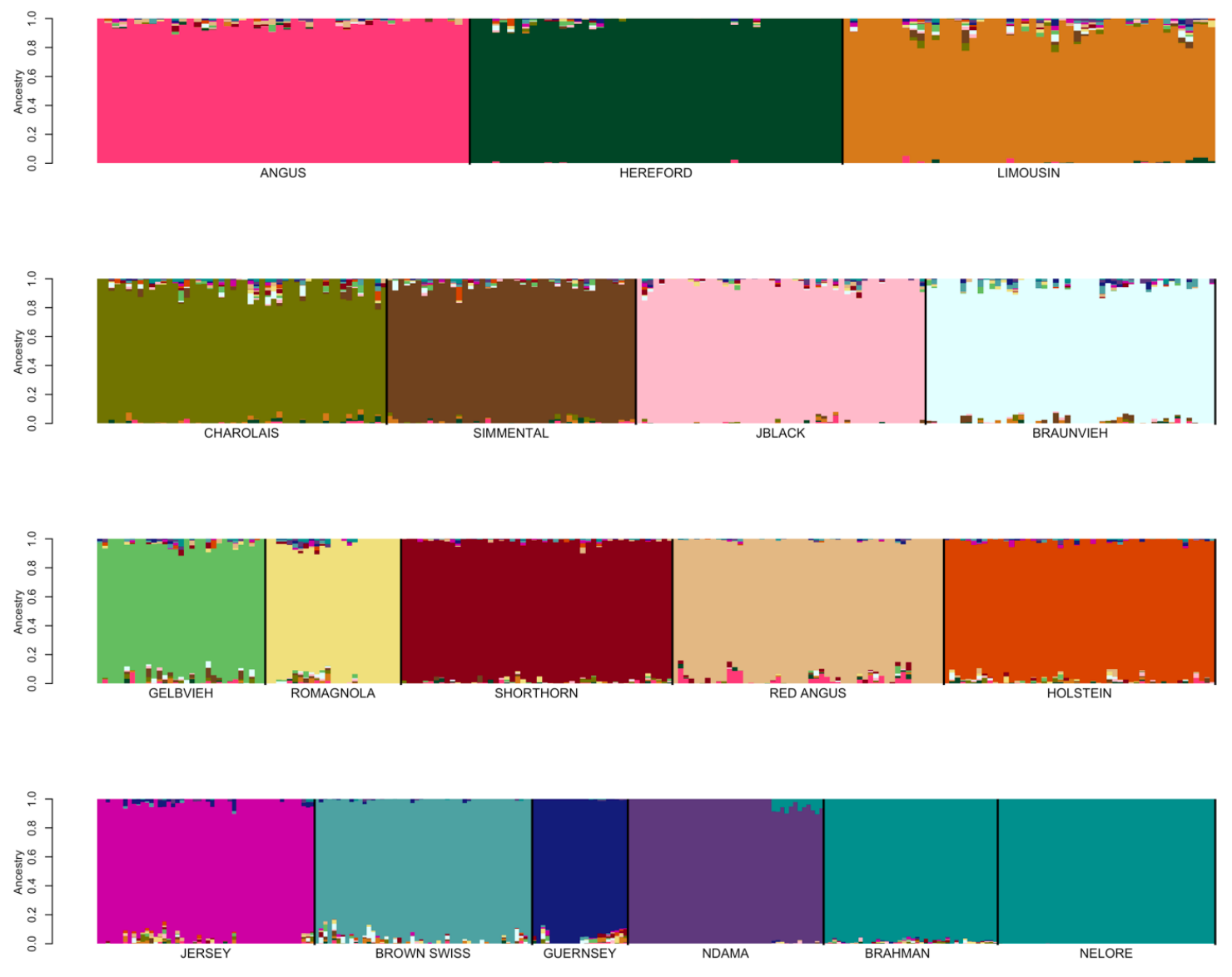

Figure 2.13. Self-assignment of ancestry for the animals in the reference breed set formed with $\leq 50$ individuals per breed from the individuals that had $\geq 85 \%$ assignment to their breed of registration using ADMIXTURE. 

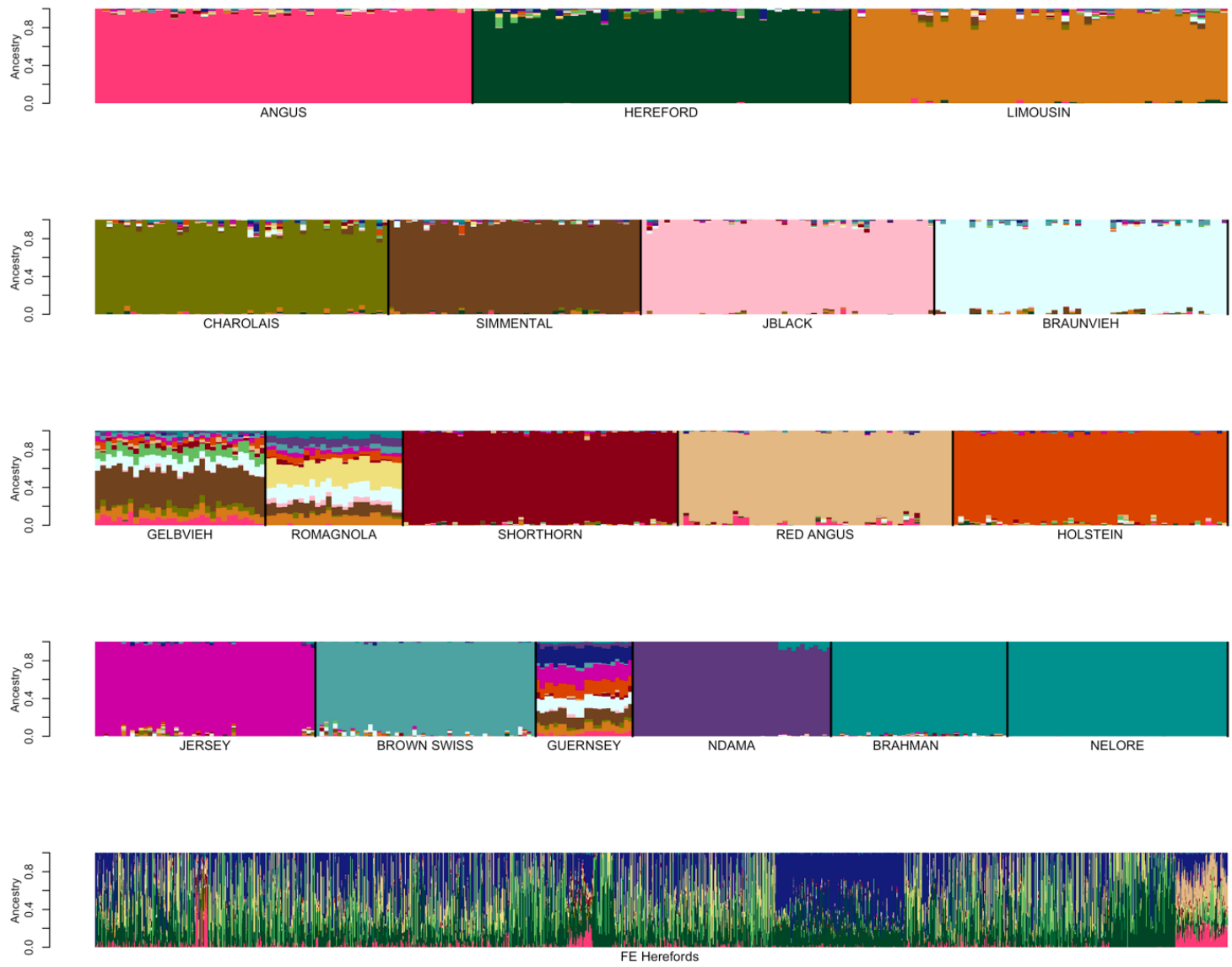

Figure 2.14. ADMIXTURE analysis conducted using the same data as shown in Figure 2.13 (first four rows), merged with an additional 2,005 high percentage crossbred Hereford target individuals (last row). Here, the 2,005 Hereford crossbred individuals appear after the reference individuals in the input genotype file. 

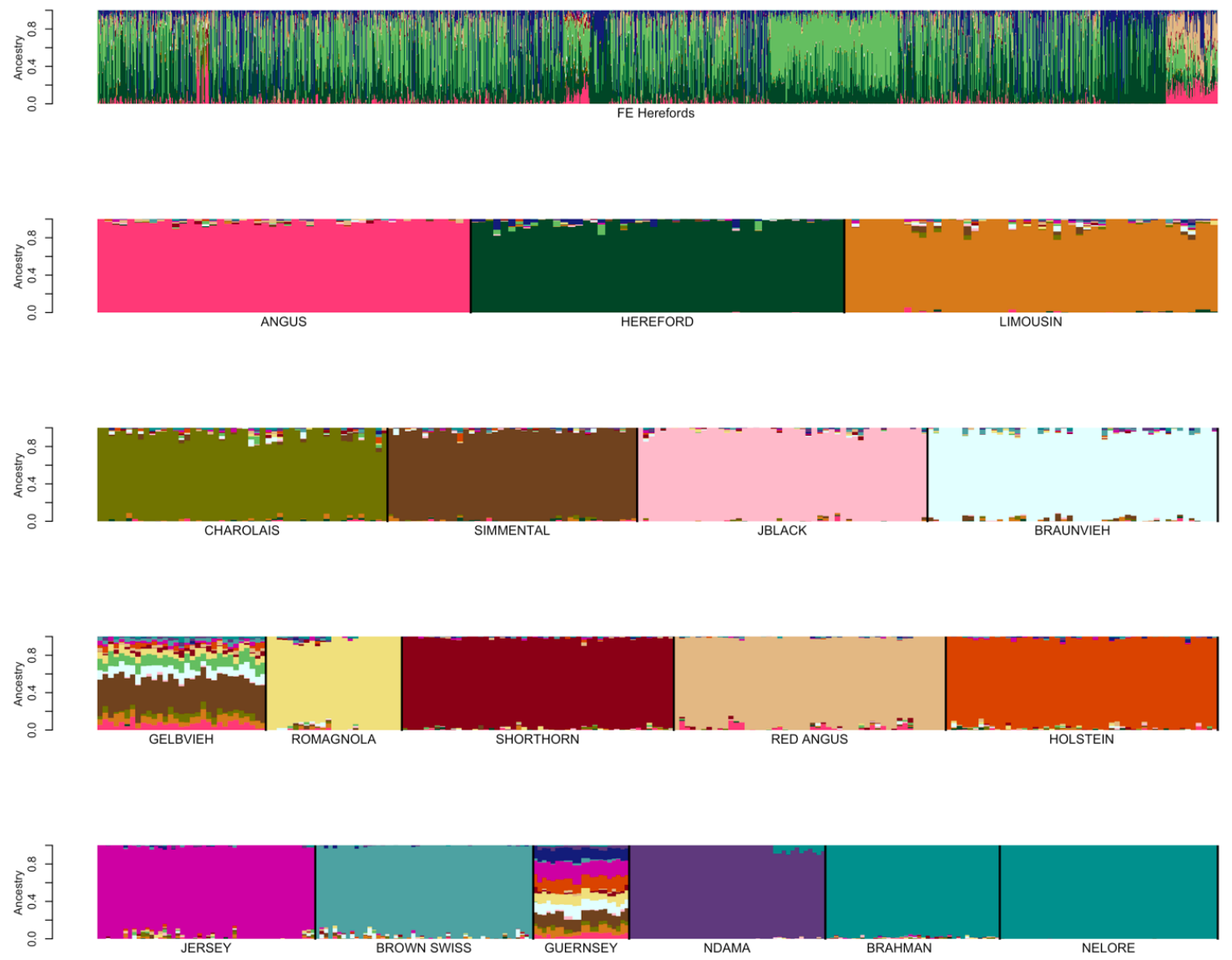

Figure 2.15. ADMIXTURE analysis conducted using the same data as shown in Figure 2.14, where the 2,005 Hereford crossbred individuals appear before the reference individuals in the input genotype file. The first row represents the 2005 Hereford crossbred samples. Rows 2 to 5 show the reference panel individuals. 

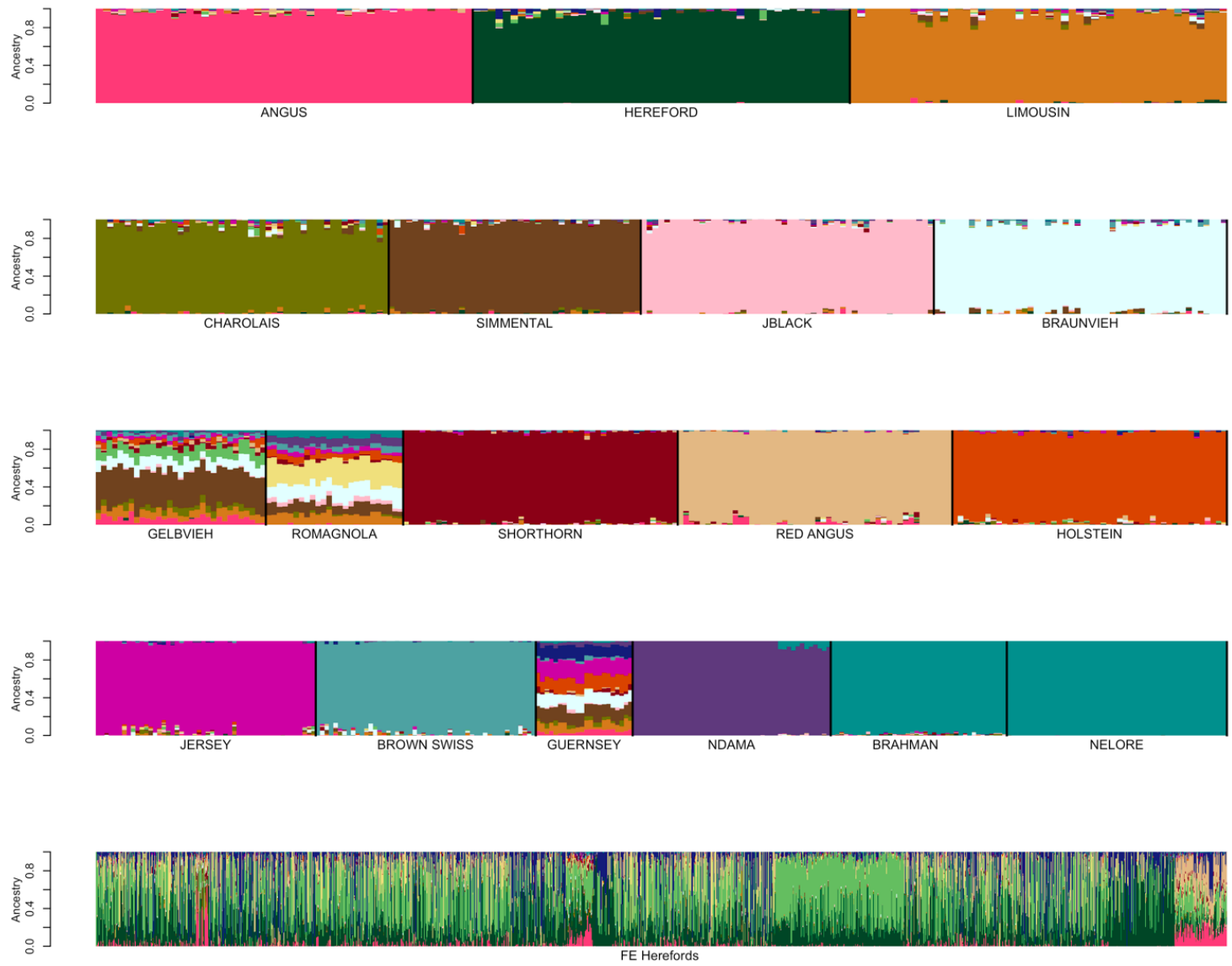

Figure 2.16. ADMIXTURE analysis conducted using the same data as shown in Figures 2.14 and 2.15, but with the order of the individuals in the input genotype file randomized. The animals were sorted following analyses to generate this figure where the first four rows represent the reference panel individuals, the fifth row shows the 2,005 Hereford crossbred animals. 

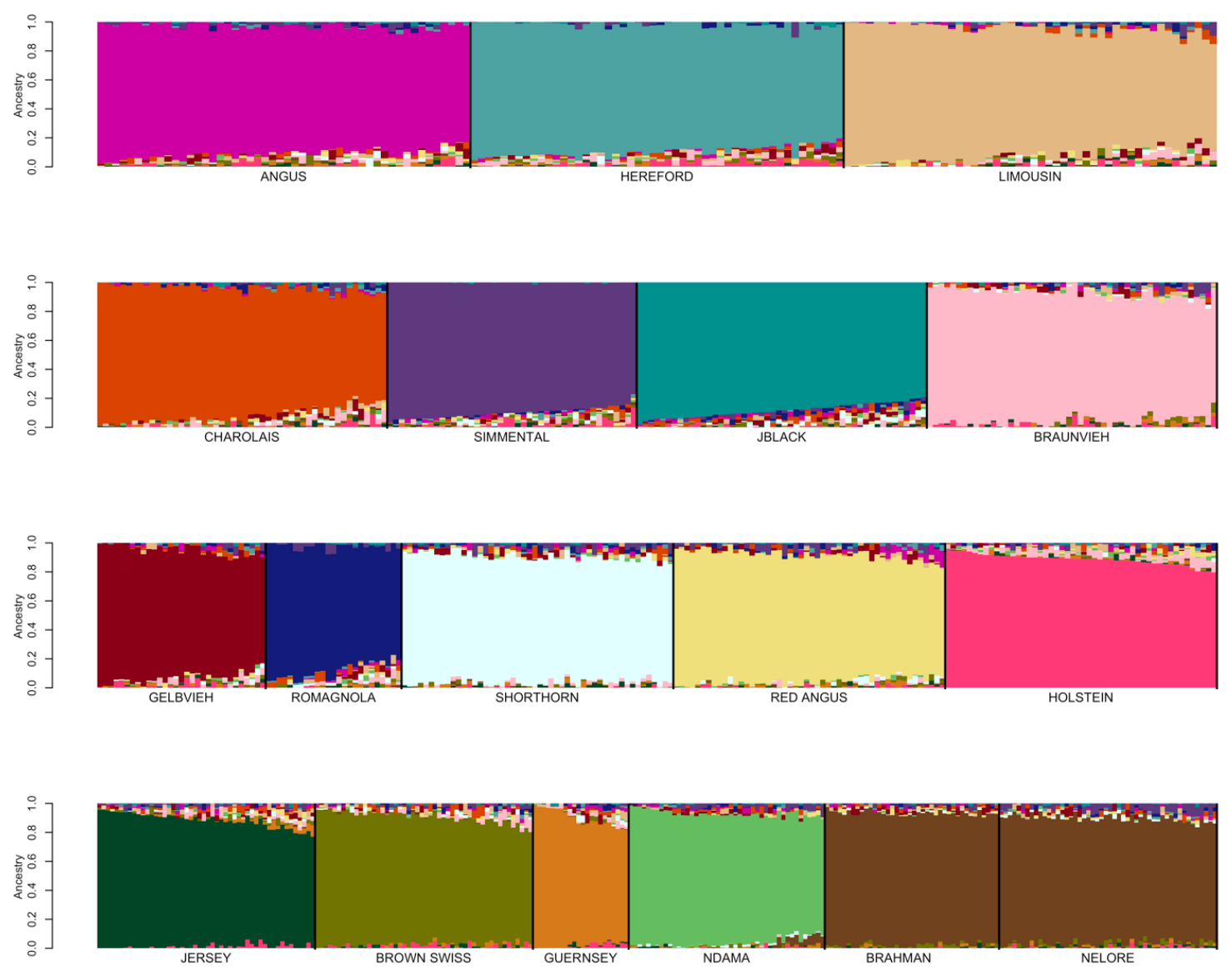

Figure 2.17. Reference breed panel constructed by the random sampling of $\leq 50$ individuals per breed from individuals with $\geq 85 \%$ ancestry was selfassigned to reference breed ancestry using the BC6K marker set. 


\section{Tables}

Table 2.1. Genotype data for 48,776 registered individuals from 20 breeds were used to establish the reference population.

\begin{tabular}{lrrrcc}
\hline Breed & $\begin{array}{c}\text { No. } \\
\text { Registered } \\
\text { Individuals }\end{array}$ & $\begin{array}{c}\text { No. Fullblood } \\
\text { Individuals }\end{array}$ & $\begin{array}{c}\text { No. } \\
\text { Individuals } \\
\text { Assigned to } \\
\text { Breed }^{\text {b }}\end{array}$ & $\begin{array}{c}\text { Sampled } \\
\text { Individuals }\end{array}$ & $\begin{array}{c}\text { No. Individuals } \\
\text { After Pedigree } \\
\text { and } \\
\text { SNPweights }\end{array}$ \\
\hline Angus & 5,552 & 5,552 & 485 & 200 & 200 \\
Hereford & 969 & 969 & 348 & 200 & 200 \\
Limousin & 2,734 & 321 & 367 & 200 & 200 \\
Charolais & 1,542 & 1,489 & 1,542 & 200 & 200 \\
Simmental & 15,858 & 337 & 1,583 & 200 & 196 \\
Japanese & 97 & 97 & 97 & 97 & 94 \\
Black & 148 & 69 & 148 & 148 & 69 \\
Braunvieh & 12,835 & 51 & 6,000 & 200 & 51 \\
Gelbvieh & 37 & 37 & 37 & 37 & 37 \\
Romagnola & 68 & 68 & 0 & 0 & 0 \\
Salers & 45 & 45 & 45 & 0 & 0 \\
$\begin{array}{l}\text { Texas } \\
\text { Longhorn }\end{array}$ & 291 & 178 & 166 & 166 & 178 \\
Shorthorn & & & & &
\end{tabular}




$\begin{array}{lrrrrr}\text { Red Angus } & 1,377 & 1,377 & 124 & 124 & 124 \\ \text { Holstein } & 5,816 & 5,816 & 5,816 & 200 & 197 \\ \text { Jersey } & 119 & 119 & 119 & 119 & 118 \\ \text { Brown } & 92 & 92 & 92 & 92 & 90 \\ \text { Swiss } & & & & & \\ \text { Guernsey } & 30 & 30 & 30 & 30 & 30 \\ \text { N'Dama } & 98 & 98 & 59 & 59 & 59 \\ \text { Brahman } & 127 & 127 & 86 & 86 & 50 \\ \text { Nelore } & 941 & 941 & 708 & 200 & 50 \\ \text { Total } & 48,776 & 17,813 & 17,852 & 2,558 & 2,143\end{array}$

$\bowtie$ aNumber of registered animals determined by pedigree analysis to be fullblood for breed associations with open herdbooks.

${ }^{b}$ Number of registered animals assigned to their identified breed with $\mathrm{P} \geq 0.97$ by fastSTRUCTURE in preliminary analyses and retained for subsequent analyses.

${ }^{\mathrm{C}} \mathrm{A}$ random sample of 200 individuals was obtained for breeds with $>200$ individuals after fastSTRUCTURE analysis and all individuals were sampled for breeds with $\leq 200$ per breed and the data were again analyzed by fastSTRUCTURE with $\mathrm{K}=19$ after removal of the Salers.

${ }^{\mathrm{d} A n i m a l s}$ that were determined to not be fullblood by pedigree analysis and animals assigned with $\mathrm{P} \leq 0.60$ by SNPweights to their breed of registry were removed. 
Table 2.2. The number of variants queried by each assay and the number of individuals from the 20 reference breeds genotyped using each assay.

\begin{tabular}{lcc}
\hline Assay & No. of Variants & $\begin{array}{c}\text { No. of Registered } \\
\text { Individuals }\end{array}$ \\
\hline BovineSNP50 & 58,336 & 20,485 \\
BovineHD & 777,962 & 2,303 \\
GGP-F250 & 227,234 & 3,068 \\
GGP-90KT & 76,999 & 4,407 \\
GGP-LDV3 & 26,504 & 6,065 \\
GGP-HDV3 & 139,977 & 3,630 \\
GGP-LDV4 & 30,105 & 8,653 \\
GGP-LDV1 & 8,762 & 165 \\
Zoetis i50K & 59,825 & 0 \\
ICBF IDBv3 & 53,450 & 0 \\
BOVGv1 & 47,843 & 0 \\
Total & & 48,776 \\
\hline
\end{tabular}


Table 2.3. Number of individuals for each reference breed assigned to their breed of registration by minimum ancestry threshold.

\begin{tabular}{|c|c|c|c|c|c|}
\hline \multirow[b]{2}{*}{ Breed } & \multicolumn{5}{|c|}{ Breed Assignment Probability } \\
\hline & $\geq 90 \%$ & $\geq 85 \%$ & $\geq 80 \%$ & $\geq 75 \%$ & $\geq 70 \%$ \\
\hline Angus & 51 & 136 & 184 & 199 & 200 \\
\hline Hereford & 58 & 136 & 184 & 200 & 200 \\
\hline Limousin & 93 & 127 & 144 & 162 & 173 \\
\hline Charolais & 52 & 92 & 119 & 132 & 147 \\
\hline Simmental & 21 & 43 & 81 & 103 & 121 \\
\hline $\begin{array}{l}\text { Japanese } \\
\text { Black }\end{array}$ & 52 & 73 & 78 & 83 & 86 \\
\hline Braunvieh & 37 & 57 & 63 & 65 & 68 \\
\hline Gelbvieh & 23 & 31 & 39 & 43 & 43 \\
\hline Romagnola & 10 & 25 & 32 & 36 & 37 \\
\hline Shorthorn & 34 & 98 & 159 & 170 & 177 \\
\hline Red Angus & 48 & 88 & 110 & 120 & 123 \\
\hline Holstein & 39 & 119 & 172 & 193 & 196 \\
\hline Jersey & 52 & 77 & 91 & 108 & 116 \\
\hline Brown Swiss & 38 & 64 & 73 & 82 & 86 \\
\hline Guernsey & 12 & 22 & 29 & 30 & 30 \\
\hline N'Dama & 27 & 45 & 59 & 59 & 59 \\
\hline Brahman & 15 & 40 & 50 & 50 & 50 \\
\hline Nelore & 32 & 50 & 50 & 50 & 50 \\
\hline Total & 694 & 1,323 & 1,717 & 1,885 & 1,962 \\
\hline
\end{tabular}


Table 2.4. Ancestry proportion statistics for the self-assignment of reference panel members from samples of $\leq 50$ or $\leq 100$ individuals from the candidate reference breed individuals.

\begin{tabular}{lcccccc}
\hline Breed & $\begin{array}{c}\text { Min } \% \\
(\leq 50)\end{array}$ & $\begin{array}{c}\text { Avg \% } \\
(\leq 50)\end{array}$ & $\begin{array}{c}\text { Max } \% \\
(\leq 50)\end{array}$ & $\begin{array}{c}\text { Min } \% \\
(\leq \mathbf{1 0 0})\end{array}$ & $\begin{array}{c}\text { Avg \% } \\
(\leq \mathbf{1 0 0})\end{array}$ & $\begin{array}{c}\text { Max } \% \\
(\leq \mathbf{1 0 0})\end{array}$ \\
\hline Angus & 86.22 & 90.40 & 95.54 & 78.49 & 87.05 & 94.13 \\
Hereford & 79.75 & 90.08 & 95.05 & 73.41 & 87.39 & 96.81 \\
Limousin & 69.52 & 88.53 & 98.16 & 18.36 & 86.40 & 98.81 \\
Charolais & 78.14 & 90.19 & 99.82 & 48.93 & 77.46 & 93.96 \\
Simmental & 81.06 & 90.37 & 97.66 & 61.36 & 73.05 & 88.11 \\
Japanese Black & 81.44 & 90.00 & 97.07 & 24.51 & 86.50 & 98.95 \\
Braunvieh & 71.59 & 89.46 & 98.61 & 65.46 & 88.36 & 98.70 \\
Gelbvieh & 73.03 & 76.27 & 81.63 & 60.92 & 74.59 & 80.33 \\
Romagnola & 75.05 & 87.18 & 96.66 & 74.79 & 85.99 & 95.12 \\
Shorthorn & 84.42 & 88.69 & 94.54 & 70.71 & 85.27 & 96.35 \\
Red Angus & 79.00 & 89.60 & 96.33 & 68.07 & 86.83 & 97.38 \\
Holstein & 85.82 & 90.30 & 97.51 & 62.95 & 86.97 & 97.81 \\
Jersey & 78.55 & 89.28 & 95.93 & 61.23 & 86.54 & 97.18 \\
Brown Swiss & 80.10 & 89.22 & 96.40 & 61.68 & 86.02 & 98.42 \\
Guernsey & 79.53 & 89.19 & 95.85 & 77.40 & 88.31 & 94.36 \\
N'Dama & 80.67 & 89.25 & 96.90 & 78.91 & 87.78 & 95.67 \\
B. t. indicus & 87.83 & 91.91 & 97.75 & 81.43 & 89.79 & 97.60 \\
\hline
\end{tabular}


Table 2.5. Average predicted ancestry and variance in predicted ancestry for candidate reference breed individuals when filtered on minimum predicted ancestry.

\begin{tabular}{|c|c|c|c|c|c|c|c|c|c|c|}
\hline Breed & $\begin{array}{l}\text { Av. \% } \\
(70 \%)\end{array}$ & $\begin{array}{l}\text { Var. } \\
(70 \%)\end{array}$ & $\begin{array}{l}\text { Av. \% } \\
(75 \%)\end{array}$ & $\begin{array}{l}\text { Var. } \\
(75 \%)\end{array}$ & $\begin{array}{l}\text { Av. \% } \\
(80 \%)\end{array}$ & $\begin{array}{l}\text { Var. } \\
(80 \%)\end{array}$ & $\begin{array}{l}\text { Av. \% } \\
(85 \%)\end{array}$ & $\begin{array}{l}\text { Var. } \\
(85 \%)\end{array}$ & $\begin{array}{l}\text { Av. \% } \\
(90 \%)\end{array}$ & $\begin{array}{l}\text { Var. } \\
(90 \%)\end{array}$ \\
\hline Angus & 86.50 & 0.21 & 87.95 & 0.19 & 87.33 & 0.22 & 88.86 & 0.13 & 72.34 & 0.97 \\
\hline Hereford & 86.99 & 0.22 & 87.09 & 0.23 & 87.48 & 0.19 & 88.25 & 0.13 & 84.62 & 0.43 \\
\hline Limousin & 86.77 & 0.55 & 89.03 & 0.44 & 87.92 & 0.38 & 88.48 & 0.43 & 80.62 & 1.19 \\
\hline Charolais & 80.18 & 2.16 & 85.03 & 1.77 & 86.28 & 0.99 & 88.56 & 0.52 & 81.54 & 0.76 \\
\hline Simmental & 72.73 & 0.89 & 78.45 & 0.58 & 83.81 & 0.36 & 89.65 & 0.15 & 87.82 & 0.50 \\
\hline Japanese Black & 87.85 & 0.52 & 88.04 & 0.39 & 88.46 & 0.27 & 88.74 & 0.21 & 80.06 & 0.61 \\
\hline Braunvieh & 87.01 & 0.37 & 87.84 & 0.36 & 87.33 & 0.38 & 88.71 & 0.21 & 80.47 & 1.24 \\
\hline Gelbvieh & 86.68 & 0.41 & 87.10 & 0.43 & 87.52 & 0.34 & 88.43 & 0.34 & 83.31 & 1.25 \\
\hline Romagnola & 86.16 & 0.33 & 86.37 & 0.32 & 87.16 & 0.32 & 86.22 & 0.29 & 86.38 & 1.16 \\
\hline Shorthorn & 85.97 & 0.26 & 87.03 & 0.22 & 86.80 & 0.14 & 87.38 & 0.07 & 83.00 & 0.70 \\
\hline Red Angus & 86.41 & 0.53 & 87.08 & 0.48 & 87.40 & 0.35 & 87.46 & 0.23 & 23.37 & 0.66 \\
\hline Holstein & 86.44 & 0.27 & 87.82 & 0.21 & 87.54 & 0.13 & 88.77 & 0.12 & 79.71 & 0.61 \\
\hline Jersey & 87.01 & 0.46 & 86.93 & 0.44 & 87.86 & 0.24 & 87.98 & 0.27 & 80.52 & 0.71 \\
\hline Brown Swiss & 86.22 & 0.47 & 86.73 & 0.51 & 88.24 & 0.26 & 88.11 & 0.20 & 82.23 & 0.70 \\
\hline Guernsey & 86.46 & 0.23 & 87.64 & 0.19 & 87.50 & 0.25 & 88.02 & 0.51 & 80.43 & 2.36 \\
\hline
\end{tabular}




\begin{tabular}{lllllllllll} 
N'Dama & 87.76 & 0.19 & 87.91 & 0.21 & 87.89 & 0.15 & 89.25 & 0.17 & 86.40 & 0.52 \\
B. $t$. indicus & 87.68 & 0.07 & 88.24 & 0.09 & 87.55 & 0.11 & 88.53 & 0.09 & 84.89 & 0.38 \\
Average & 85.58 & 0.48 & 86.84 & 0.41 & 87.30 & 0.30 & 88.32 & 0.24 & 78.69 & 0.87 \\
\hline
\end{tabular}


Table 2.6. Average breed ancestry percentages assigned to American Breed individuals.

\begin{tabular}{lccc}
\hline Breed & $\begin{array}{c}\text { Av. Ancestry } \\
\text { Beefmaster } \% \\
( \pm \text { st. dev })\end{array}$ & $\begin{array}{c}\text { Av. Ancestry } \\
\text { Brangus \% } \\
( \pm \text { st. dev })\end{array}$ & $\begin{array}{c}\text { Av. Ancestry } \\
\text { Santa Gertrudis \% } \\
( \pm \text { st. dev })\end{array}$ \\
\hline Angus & $3.29( \pm 4.27)$ & $32.15( \pm 8.96)$ & $4.90( \pm 4.48)$ \\
Hereford & $16.13( \pm 2.83)$ & $2.03( \pm 2.93)$ & $2.50( \pm 4.05)$ \\
Limousin & $1.40( \pm 2.28)$ & $1.73( \pm 2.56)$ & $1.29( \pm 2.19)$ \\
Charolais & $6.89( \pm 3.97)$ & $2.07( \pm 3.79)$ & $5.26( \pm 3.42)$ \\
Simmental & $2.65( \pm 3.12)$ & $1.16( \pm 2.92)$ & $0.40( \pm 1.40)$ \\
Japanese Black & $0.53( \pm 3.46)$ & $0.10( \pm 0.63)$ & $0.22( \pm 0.89)$ \\
Braunvieh & $0.63( \pm 1.64)$ & $0.33( \pm 1.29)$ & $0.59( \pm 1.63)$ \\
Gelbvieh & $3.19( \pm 3.30)$ & $3.14( \pm 3.67)$ & $2.59( \pm 3.20)$ \\
Romagnola & $1.05( \pm 1.94)$ & $0.54( \pm 1.39)$ & $0.68( \pm 1.57)$ \\
Shorthorn & $15.36( \pm 4.72)$ & $5.86( \pm 3.42)$ & $37.71( \pm 5.46)$ \\
Red Angus & $3.66( \pm 3.57)$ & $13.60( \pm 3.95)$ & $1.18( \pm 3.46)$ \\
Holstein & $6.22( \pm 6.73)$ & $4.53( \pm 4.82)$ & $0.89( \pm 2.83)$ \\
Jersey & $0.73( \pm 1.65)$ & $0.52( \pm 1.37)$ & $0.26( \pm 1.08)$ \\
Brown Swiss & $1.05( \pm 2.14)$ & $1.28( \pm 2.26)$ & $0.73( \pm 1.81)$
\end{tabular}


Guernsey

N'Dama

B. t. indicus
$1.53( \pm 2.20)$

$0.52( \pm 1.35)$

$27.32( \pm 4.84)$
$0.17( \pm 0.81)$

$0.19( \pm 0.87)$

$23.09( \pm 6.73)$
$1.50( \pm 2.14)$

$0.16( \pm 0.76)$ 


\title{
CHAPTER 3 \\ HAPLOTYPIC MOSAICS OF ADVANCED GENERATION COMPOSITE BREED CATTLE FORMED AS CROSSES BETWEEN TAURINE AND INDICINE
}

\begin{abstract}
Background

The American breeds of beef cattle began to be developed in the 1920 s as Bos taurus taurus $\times$ Bos taurus indicus hybrids that contain pre-specified amounts of Brahman influence. By 1954, Breed Associations had been formed for the Brangus, Beefmaster, and Santa Gertrudis breeds. While the American breeds were developed to meet specific breed compositions based upon pedigree, they are now advanced generation composites and selection or drift may have caused the actual genome compositions of these breeds to differ from the expected pedigree proportions specified by the respective breed association. The availability of high-density SNP genotypes for relatively large samples of animals from these breeds now allows the opportunity to evaluate the genomic and chromosomal architectures of these breeds relative to expectation based on breed specifications.
\end{abstract}

\section{Results}

Using the RFmix method to estimate local ancestry, we identified regions of the genome that differed significantly from a priori composition expectations, both within and across these American breed populations. For all three American 
breeds, higher than expected levels of Brahman composition were found on chromosome 5 which has previously been identified as harboring loci related to disease and parasite resistance and Brahman phenotypic characteristics. Differences in regional composition within the breeds were also detected, suggesting differences in selection pressures and breeding objectives between the breeds.

\section{Conclusions}

Global ancestry estimation is commonly performed in livestock to establish the relationship between populations and control for stratification in association studies. However, local ancestry estimation provides the opportunity to investigate the evolution of specific chromosomal segments and their effects on trait variation within admixed individuals, information that is averaged across loci with global ancestry estimation. Investigation into the genomic architecture of American breeds could provide information regarding the genetic locations of selected loci and individual specific genotype information that could be used in genome-wide association analyses to identify loci with large breed-specific effects and dominance effects potentially underlying heterosis in production traits of admixed cattle.

\section{Background}

Indicine cattle were first imported into the United States from India in 1906 and then from Brazil in the 1920s and were used via crossbreeding with taurine cattle 
and backcrossing to develop the Bos taurus indicus Brahman [83] which has very little residual Bos taurus taurus within its genome [89]. The American breeds of beef cattle are populations that were developed in the United States beginning shortly after the introduction of the B. t. indicus cattle to capitalize on their adaptive advantages for cattle production in the southern tier of the country. Breed associations for the American breeds began to be formed in the 1940s and advanced generation composite animals now exist for the older Brangus, Beefmaster, and Santa Gertrudis breeds. These breeds were developed to have different proportions of B. t. taurus and B. t. indicus across the breeds, but individuals within each breed are expected to have the same taurine and indicine proportions based on pedigree expectations.

B. t. taurus and B. t. indicus are subspecies that are the result of two different domestication events in the Fertile Crescent and Indus Valley, respectively, approximately 10,000 years ago [90]. The migration of humans throughout Europe and Asia, led to the geographical isolation of small groups of herded cattle, ultimately leading to the development of geographically isolated populations. Later, these isolated populations became the founding members of distinct cattle breeds $[37,38]$. Breeds of cattle have been selected for a number of purposes and phenotypes, including distinct coat colors, presence or absence of horns, and for specialized meat or milk production. Following the development of artificial insemination in the 1940s, intense selection allowed a rapid differentiation of breeds based on the selected traits and some registered breeds 
have capitalized on the effects of heterosis and breed complementarity to improve production by crossbreeding [91, 92].

The divergence between $B$. t. indicus and B. t. taurus has been estimated to be approximately 250,000 years and substantial hybrid vigor, or heterosis, is observed when crossbred individuals are produced from these two subspecies. Indicine-taurine cross individuals have been produced in subtropical and tropical regions world-wide to combine the superior temperate environment production attributes of taurines with the adaptive advantages of indicines to the nutritional limitations of harsh climates and resistance to parasites and disease-causing pathogens $[93,94]$. The American breeds were developed to capitalize on these advantages and to create breeds in which advanced generation animals had stable indicine and taurine genome proportions. For example, Beefmasters were developed by an initial cross between Brahman, Hereford (B. t. taurus), and Shorthorn ( $B$ t. taurus) in 1908 and are now maintained at expected pedigree proportions of $50 \%, 25 \%$, and $25 \%$ inheritance, respectively [95]. Similarly, Brangus cattle were derived from animals created in public and private breeding experiments involving crosses of Angus (B. t. taurus) and Brahman cattle in Oklahoma, Mississippi, Texas and Louisiana in the 1930s and have been stabilized at expected genome contents of $3 / 8$ Brahman and 5/8 Angus [http://afs.okstate.edu/breeds/cattle/brangus/index.html/]. Santa Gertrudis cattle were initially developed at the King Ranch in Texas where experimental crossbreeding between Shorthorn and Brahman cattle between 1910 and 1920 
led to the birth of the bull "Monkey" from which all registered Santa Gertrudis cattle descend [http://afs.okstate.edu/breeds/cattle/santagertrudis/index.html]. In 1940, the United States Department of Agriculture recognized Santa Gertrudis as a $3 / 8$ Brahman and 5/8 Shorthorn beef breed [http://santagertrudis.com/history.html]. These American breeds of cattle now provide an interesting opportunity to study the genomic architectures of advanced generation composites with known expected genomic breed proportions based on pedigree that have undergone natural selection for adaptation and artificial selection for performance traits.

Several approaches have been developed for the estimation of local ancestry (breed of origin of the two alleles present at specific loci) in admixed individuals, however, these applications have primarily been focused on recently admixed populations. Individuals from admixed populations have chromosomes that comprise mosaics of chromosomal segments originating from each of the ancestral populations [96]. On the other hand, global ancestry estimates the relative proportions of ancestral chromosome segments present within an individual throughout the entire genome, or an average of the local ancestry estimates, thereby losing information pertaining to the variability of locus specific ancestries [97]. Mendelian sampling, the random assortment of recombinant chromosomes into gametes, and strong selection can lead to regions of the genome with ancestries that differ significantly from breed expectation and 
examination of these regions may identify candidate genes that are under selection and suggest the nature of the selected phenotype.

We estimated local ancestry for registered Brangus, Beefmaster, and Santa Gertrudis animals that had been genotyped with the BovineSNP50 or derivative assays and examined the average ancestries at specific chromosomal locations in an attempt to identify regions of the genome that differ from expected global proportions both within and across breeds.

\section{Materials and Methods}

Genotype data for advanced generation composite animals from the Brangus, Beefmaster, and Santa Gertrudis breeds were obtained from the respective Breed Associations (Table 3.1). These individuals had been genotyped using at least one of 7 commonly used genotyping platforms including the GeneSeek (Lincoln, NE) GGP-90KT, GGP-F250, GGP-HDV3, and GGP-LDV3 assays, the Illumina (San Diego, CA) BovineHD and BovineSNP50 assays, and the Zoetis (Kalamazoo, MI) i50K assay. PLINK1.9 [77] was used to filter variants and individuals. The SNP positions were based on the ARS-UCD1.2 bovine reference genome assembly [98]. Non-autosomal variants were removed from the data. Variants and individuals with genotype call rates $<0.90$ were also removed. Genotypes were phased using Eagle 2.4 [99] with a reference panel of haplotypes for 9,937 individuals genotyped with the Illumina BovineHD assay (Illumina, San Diego, CA). Phased haplotypes were then imputed with Minimac3 
[100] to the union of two high-density research assays; the Illumina BovineHD (HD) and the GeneSeek GGP-F250 (F250). The multi-breed reference set created by Rowan et al. (2019) [101] was used for genotype imputation. The reference panel contained 2,719 animals that had been genotyped with both the F250 and the HD assays, 25,772 animals genotyped with only the F250, and 7,218 animals genotyped with only the HD assay. Following imputation, each sample had genotypes for 836,118 variants.

Local ancestry estimation requires the use of a reference population. For this study, we developed two reference panel populations based on different selection criteria for evaluation. One reference panel was constructed using animals with CRUMBLER breed composition estimates of $\geq 85 \%$ assignment to Angus, Hereford, Shorthorn, and Brahman, respectively (henceforth referred to as the CRUMBLER reference) [68] (Table 3.2). The second reference panel was created based upon the total number of registered Angus, Hereford, Shorthorn, and Brahman animals (henceforth referred to as the EXPANDED reference). Because some ancestry estimation software is influenced by unequal reference panel sample sizes $[68,102]$, we identified the foundation breed with the fewest registered animals for each of the American breeds and then sampled the other foundation breeds to have approximately the same numbers of sampled animals (Table 3.2). Shorthorn had the fewest samples with 183 and 487 individuals in the CRUMBLER and EXPANDED reference panels, respectively. Potential reference panel genotypes were genotyped using 8 commercially available 
bovine assays including the GGP-F250, GGP-HDV3, GGP-LDV3, BovineHD, BovineSNP50, i50K, Irish Cattle Breeding Federation (Cork, Ireland) IDBv3, and GeneSeek (Lincoln, NE) BOVG50v1 and were phased and imputed following the same procedures as for the American breed samples.

Local ancestry software, RFMIX v2.03, was used for local ancestry and admixture estimation [102]. RFMIX is a discriminative approach, modeling the dependence of unobserved variable $\mathrm{Y}$ on a given observed variable $\mathrm{X}$ directly, which in the case of RFmix provides rapid and robust local ancestry and admixture predictions for a set of unknown samples, or more specifically a set of contiguous disjoint windows from an admixed chromosome, based on known and provided reference samples [102]. The method partitions chromosomes into windows and infers the ancestry for each chromosomal segment using a random forest algorithm parameterized by conditional random fields (CRFs) trained on a user specified reference panel [102]. We inferred local ancestry using RFmix v2.03, with a CRF window of 100 SNPs. This requires RFmix to use 100 SNPs per non-overlapping window for ancestry estimation, however, if successive windows are assigned the same ancestry, RFmix combines the windows resulting in CRF windows of $>100$ SNPs. RFmix allows the specification of the number of generations separating the query samples since the initial admixture of the reference populations. However, since pedigree information was not provided by the American breed Breed Associations, we used a generation interval of 5 years and the dates of formation of the breed associations to arrive 
at a maximum of 16 generations, but likely 8 generations, on average, for these samples. This reflects the fact that American breed cattle are continuously formed and registered by breeders using superior animals from the foundation breeds to capture the benefits of on-going selection within these numerically large breeds.

Due to the lack of a priori pedigree information for the genotyped animals, initial genome-wide ancestry was evaluated through RFmix. Many of the American breed Breed Associations allow the registration of purebred founding breed parents and $F_{1}$ individuals within their respective herdbook. To alleviate concern of such animals being evaluated as advanced generation composites, we removed samples that were assigned to be $\geq 50 \%$ Brahman or $\geq 90 \%$ Angus or Shorthorn ancestry for the Brangus and Santa Gertrudis populations, respectively (Brangus, $n=297$ removed; Santa Gertrudis, $n=4$ removed) (Figures S3.1 and S3.3). For the Beefmaster population, which was formed as a 3-way cross, we removed samples with $\geq 90 \%$ assignment to Shorthorn, Hereford or Brahman to remove any potentially genotyped purebred founders or with $\leq 5 \%$ assignment to any one breed to remove any potential $F_{1}$ cross individuals ( $n=10$ removed) (Figure S3.2).

Regions of significant divergence from expected Brahman proportion were evaluated using Benjamini-Hochberg adjusted p-values. P-values were calculated using a t-statistic for each CRF window with the equation: 


$$
-a b s\left(\frac{p i-A v g(p i)}{\sqrt{\operatorname{Var}(p i)}}\right)
$$

where $p i$ is the proportion of Brahman ancestry estimated for window $i$. Average $p i$ and standard deviation of $p i$ were computed to represent the genome-wide average and standard error of Brahman proportion for each population (genomewide averages and standard errors are reported in Table 3.4). P-values were computed assuming a t-distribution and were then corrected for multiple testing using the Benjamini-Hochberg method to address the issue of false positives . Significant regions were then extracted based on selected Benjamini-Hochberg adjusted P-value thresholds and the regions were intersected with QTL reported in the Cattle dbQTL [103].

\section{Results and Discussion}

The Santa Gertrudis breed was developed as a result of a cross between Brahman males and Shorthorn females on the King Ranch in Kingsville, TX. However, the initial formation of the breed used $F_{1}$ crosses in which the Brahman bulls that were used actually ranged in composition from $3 / 4$ to $7 / 8$ B. t. indicus and, as a consequence, the Santa Gertrudis breed is considered to have a composition of $3 / 8$ Brahman and $5 / 8$ Shorthorn. The first exploratory crosses of Brahman bulls and Shorthorn females began in 1918, however, Santa Gertrudis was not recognized as a breed by the United States Department of Agriculture until 1940 [95, 104]. Beefmaster was developed beginning in 1908 as cross of Brahman, Shorthorn, and Hereford on the Lasater Ranch in Falfurrias, TX [95]. 
The Beefmaster was recognized as a beef breed by the United States Department of Agriculture in 1954. The breed has an expected composition of 25\% Hereford, 25\% Shorthorn, and 50\% Brahman. The Brangus breed originated in 1949 with the formation of the American Brangus Breeders Association. However, the first crosses of Brahman and Angus animals began as early as 1912. Registered Brangus individuals must be $5 / 8$ Angus or Red Angus and $3 / 8$ Brahman descent. They must also possess a solid red or black coat color and the polled phenotype.

RFmix analysis was conducted using both the CRUMBLER and EXPANDED reference panels. RFmix provides three outputs for each analysis: (1) the most likely reference population assignments for each haplotype defined by a CRF window (i.e., CRF window is assigned to a reference population), (2) the marginal probabilities of each reference population being the ancestral population of haplotypes for the CRF window, and (3) global diploid ancestry estimates [102]. For the analyses performed here, we were particularly interested in the proportions of each foundation breed haplotypes that were found at each chromosomal location throughout the genome for each of the American breeds. To accomplish this, for each CRF window we determined the proportion of haplotypes corresponding to each reference population by obtaining the frequency of haplotypes assigned to a reference population for a given CRF window as found in the RFmix most likely assignment output. The frequency was averaged across the total number of haplotypes present in the sample (i.e., 
the 9,161 Brangus individuals had 18,322 haplotype calls per CRF window) (Figures 3.1-3.3) to generate an overall population proportion of haplotypes corresponding to each reference population.

Additionally, we evaluated the distribution of marginal probabilities of each reference population being the ancestral population for each CRF window (Figures S3.4-3.6). These probability distributions indicate that RFmix was able to identify ancestry with very high probabilities since a small proportion of assignment probabilities were $0.05<\mathrm{P}<0.95$ (Table 3.3). This would be expected for admixture between very highly diverged populations for which there was a very limited extent of haplotype sharing.

\section{Effect of Reference Panel Size}

Reference panel sample sizes have been shown to have significant effects on the accuracy of RFmix estimates [102]. To our knowledge, this is the first application of RFmix in bovine, and as such, a priori knowledge concerning appropriate reference panel sample sizes necessary for local ancestry estimation have not been published. Reference panel sizes for analyses of human local ancestry have ranged from as few as 19 samples to more than 500 samples per population $[102,105]$ and the human effective population size has been estimated to be in the range from 3,100 for Europeans to 7,500 for Yorubans [106]. In cattle, the effective population size of most taurine breeds has been estimated to be about 100 [54] and we might expect that smaller reference 
populations would be necessary to capture the haplotypic diversity within cattle breeds than human populations. However, random samples of 200 individuals may not be sufficient to capture the haplotypic diversity within cattle and larger sample sizes would be expected to capture more of the rare haplotypes leading to a greater accuracy of local ancestry estimation in admixed individuals [102].

Similar to the selection of reference panel individuals by Browning et al. [18], we selected two reference panels to evaluate the effect of panel size on local ancestry estimation. First, we selected the CRUMBLER [68] reference panel which had been extensively developed and evaluated for use in global ancestry estimation for breeds commonly found in North America. Samples in this panel were required to have been assigned to their breed of registry by the CRUMBLER pipeline with probabilities of $\geq 85 \%$ assignment for Angus, Hereford, Shorthorn, and Brahman (Table 3.2, Figures 3.4-3.6). The expanded reference panel was created from all of the available animals registered with their respective Breed Associations for Angus, Hereford, Shorthorn, and Brahman (Table 3.2, Figures 3.1-3.3). To avoid issues potentially caused by unequal sample sizes, we down-sampled the larger populations to the sample size for the breed with the fewest available samples (Table 3.2).

The EXPANDED reference panel, was approximately twice the size of the CRUMBLER reference panel, however, we observed only small differences between ancestry estimates produced using the two panels (Figures S3.7-S3.9). 
However, the EXPANDED reference panel enabled RFmix to more accurately recapitulate the pedigree-based proportions expected within Beefmaster (Figure S3.8). Specifically, the proportion of Hereford ancestry was underestimated and the proportion of Shorthorn ancestry was overestimated when the CRUMBLER reference panel was used for local ancestry estimation in Beefmaster. This may have been due to the extent of representation of horned Hereford and Line 1 Hereford genetics within the CRUMBLER breed composition pipeline which appears to not have captured the haplotypic diversity originating from Hereford in the Beefmaster animals.

\section{Genome-Wide Global Ancestry By Individual}

While we were particularly interested in population level deviations from Brahman genome expectation, we evaluated the data for each of the American breeds on an individual basis. Genome-wide, each of the American breeds has a lower proportion of Brahman represented within the genome than expected based on expected pedigree proportions (Figures 3.7-3.9). Differences were also found between breeds by chromosome, however the overall landscape reflects the local ancestry estimations depicted in Figures 3.1-3.3. Differences amongst individuals within each population may be due to recurrent selection and would therefore be expected to be associated with the number of generations that an individual was removed from its fullblood Angus and Brahman ancestors (generation number). In this case, stratifying the genotyped animals according to their generations numbers and testing for trends with the estimated reference 
breed proportions within each individual would enable this hypothesis to be tested; however, we did not have access to the pedigree data for these animals.

\section{Local Ancestry Estimation By American Breed}

$\underline{\text { Similarities across American breeds }}$

Genome-wide, each of the American breeds has a lower proportion of Brahman represented within the genome than expected based on pedigree proportions, estimated at $25.68 \%, 27.81 \%$, and $30.84 \%$ Brahman for the Brangus, Santa Gertrudis, and Beefmaster populations, respectively (Figures 3.1-3.3, 3.10-3.12, Table 3.4). This is unlikely to have been due to drift, but probably reflects the effects of selection for polygenic performance traits for which taurine alleles are favorable. On the other hand, we also observe a significant genomic region enriched for Brahman ancestry in all three of the breeds, suggesting that selection is acting on alleles in this region that confer an adaptive advantage

(Figures 3.13-3.15). The region located on chromosome 5 has previously been identified as harboring loci associated with penile sheath, coat color, yearling weight, body condition score, and parasite resistance in tropically adapted indicine $\mathrm{x}$ taurine crossbred cattle [93]. As the global proportion of indicine ancestry increases in B. t. taurus $\times$ B. t. indicus hybrids, coat color lightens, males tend to have more pendulous sheaths, body weight and condition scores increase, and tick and worm burdens decrease [93]. B. t. indicus cattle tend to have a lower performance than B. t. taurus cattle under favorable conditions, but significantly outperform B. t. taurus cattle in extreme climates and environments 
where parasites, heat, and low inputs play important roles in the production system [93, 107-109]. Heterosis for production and adaptive traits is considerable in B. t. taurus $\times$ B. t. indicus hybrids and breed complementarity, the combining of desirable traits originating in different parental lines, and heterosis were the factors underlying the development of the American breeds of cattle.

\section{Differences between the American breeds}

We also observed breed specific regions in which the proportion of indicine alleles differed from pedigree expectations. Brangus cattle are required to be polled and to have solid black or red coat colors. Brahman cattle are primarily genetically horned while Angus are fixed for the autosomal dominant Celtic polled allele, a complex structural insertion, located near the centromere of BTA1 $[87,110-112]$. Brahman cattle have complex coat colors that can range from solid gray to brindled, while the red and black coat color phenotypes in Angus cattle are caused by variation within $M C 1 R$ located at $14.7 \mathrm{Mb}$ on BTA18 on the ARS-UCD1.2 assembly. Figure 3.1 reveals the near fixation of Angus alleles at both loci in Brangus. Figure 3.3 shows the near fixation of Shorthorn alleles at $M C 1 R$ in Santa Gertrudis cattle presumably in response to selection for a solid red coat color.

Piebald phenotypes, or white spotted coat color patterns, are distinguishing characteristics of some cattle breeds. Evidence of piebaldism has been recorded in B. t. taurus, B. t. indicus, and crossbred cattle $[113,114]$. In human, 
piebaldism is an autosomal dominant trait that is characterized by a lack of pigmentation of the skin or hair, most commonly on the head, ventral chest/abdomen and the extremities [115]. In Hereford and Simmental cattle the piebald phenotype is also autosomal dominant and is characterized by white on the head, ventral areas, lower legs, and tail switch [113]. The Hereford and Simmental phenotypes are caused by structural variation located proximal to KIT at $70.15-70.26 \mathrm{Mb}$ on chromosome $6[113,116]$. KIT is a mast cell and stem cell growth factor receptor that mediates melanocyte development and migration [113] and is known to be associated with several white patterning phenotypes in other species [117-121]. In cattle, QTL associated with variation in white spotting have been identified on chromosome 6 near KIT, chromosome 18 and chromosome 22 in a crossbred $F_{2}$ population of Holstein-Friesian and Jersey animals [117]. The proportion of Hereford present within Beefmaster cattle is clearly reduced in the vicinity of $K I T$ (Figure 3.2). This suggests selection against the spotted phenotype in Beefmaster possibly to reduce economic losses due to ocular squamous cell carcinoma, or cancer eye, which is most common in animals that lack pigment around their eyes [31, 32].

The Shorthorn breed is characterized by variable coat color patterns, ranging from solid red to solid white, with an intermediate pattern, roan, a red coat with white patches [113]. This phenotype is caused by variation at KITLG at $18.24-$ 18.32 Mb on chromosome 5 [122]. While the signatures of selection on chromosome 5 appear to complex in the American breeds, the proportion of 
Shorthorn within the genome of Beefmaster animals is significantly reduced in the first $50 \mathrm{Mb}$ relative to the remainder of the chromosome suggesting selection to remove the Shorthorn white coat color allele from the Beefmaster population (Figure 3.2).

\section{Annotated regions significantly diverged from expected Brahman proportion} We extracted the 5 most significant regions by chromosome with a BenjaminiHochberg adjusted $p$-value $\leq 0.4$ for each population (Table S3.1 and Figures 3.13-3.15). We intersected these diverged regions with QTL locations within the cattle dbQTL [20] to evaluate whether selection may have been responsible for divergence of genome composition within these regions. The region on chromosome 5 (Figure 3.13-3.15 and Table 3.5), spanning 47.21-49.52 Mb was highly significant in all three American breed populations. This region has been identified as harboring loci associated with reproductive traits, such as interval to first estrus after calving and age at puberty in a tropically adapted composite breed heifer population [42], as well as milk composition, meat, and carcass traits. Perhaps most notable is a previously identified association with variation in this region with susceptibility to bovine respiratory disease. Indicine cattle are known for their increased tolerance to harsh environments, diseases, and parasites $[8,9]$ and these characteristics motivated the initial development of the American cattle breeds. 
Among the most significant regions deviating from Brahman expectation in the Beefmaster are on chromosomes 6, 8, and 25 (Table 3.6 and Table S3.1). These regions are enriched for QTL influencing reproductive traits as well as meat and carcass quality traits, such as fat thickness, yield grade, and marbling score which greatly affects the tenderness and the flavor of beef [123-126]. These traits are strongly selected by U.S. commercial cattle breeders because they are among the largest drivers of income and significantly affect profitability. QTL for growth traits, in particular average daily gain on chromosome 6 [127] and residual feed intake on chromosome 8 [128] have previously been identified and are located within these Brahman diverged regions in the Beefmaster.

Regions on chromosomes 20 and 21 were among the most significant for divergence from Brahman expectation in the Brangus (Table 3.7 and Table S3.1). Many associations that affect reproduction, body conformation, growth, carcass characteristics and meat quality have previously been identified within the region on chromosome 20. Associations with milk and udder characteristics have also been identified on both chromosomes 20 and 21 . Nine previously published QTL are located within the regions on chromosomes 20 and 21. QTL associated with birth, weaning, yearling, and carcass weights are located within the region on chromosome $20[129,130]$ with an association for average daily gain within the region on chromosome 21 [166]. Additionally, a QTL associated with heat tolerance has been identified in the region on chromosome 20 
suggesting selection for alleles conferring tolerance to heat and adaptation to harsh climates which are characteristics of Brahman cattle [131].

The Brahman diverged region on chromosome 20 in Santa Gertrudis (Table 3.8 and Table S3.1) harbors previously identified QTL for calving ease and carcass weight $[130,132]$. Additionally, an association with interval to first estrus after calving in this genomic region has been identified in a tropical composite population [133], as well as carcass and milk composition associations. The smaller number of genomic regions that were found to be diverged from Brahman expectation in the Santa Gertrudis may reflect the infancy of the use of selection tools such as Expected Progeny Differences (EPDs) within this breed compared to the other American breeds.

\section{Conclusions}

The American breeds are advanced generation B.t.taurus $\times$ B. t. indicus hybrid cattle that were developed to capitalize on breed complementarity and heterosis to produce cattle that are suited to harsh, subtropical climates as well as disease and parasite threats, while still maintaining acceptable levels of growth and productivity. The breeds employed mating systems designed to produce cattle that were either $5 / 8$ taurine and $3 / 8$ indicine (Brangus and Santa Gertrudis) or 1/2 taurine and $1 / 2$ indicine (Beefmaster). These cattle now allow the opportunity to examine the genomic consequences of hybridization in the presence of natural and artificial selection. We found strong evidence that selection has produced 
cattle with smaller than expected indicine compositions in the genomes of all three breeds indicating that the optimal animal adapted to production in the southern tier of the United States comprises less than $37.5 \%$ of its genome from indicine origin. We also found strong signatures of selection in genomic regions harboring Mendelian loci affecting morphological phenotypes such as the presence or absence of horns and coat color, as well as numerous QTL influencing economically important quantitative traits within regions that were found to be significantly diverged from the expected Brahman proportion. 


\section{Figures}
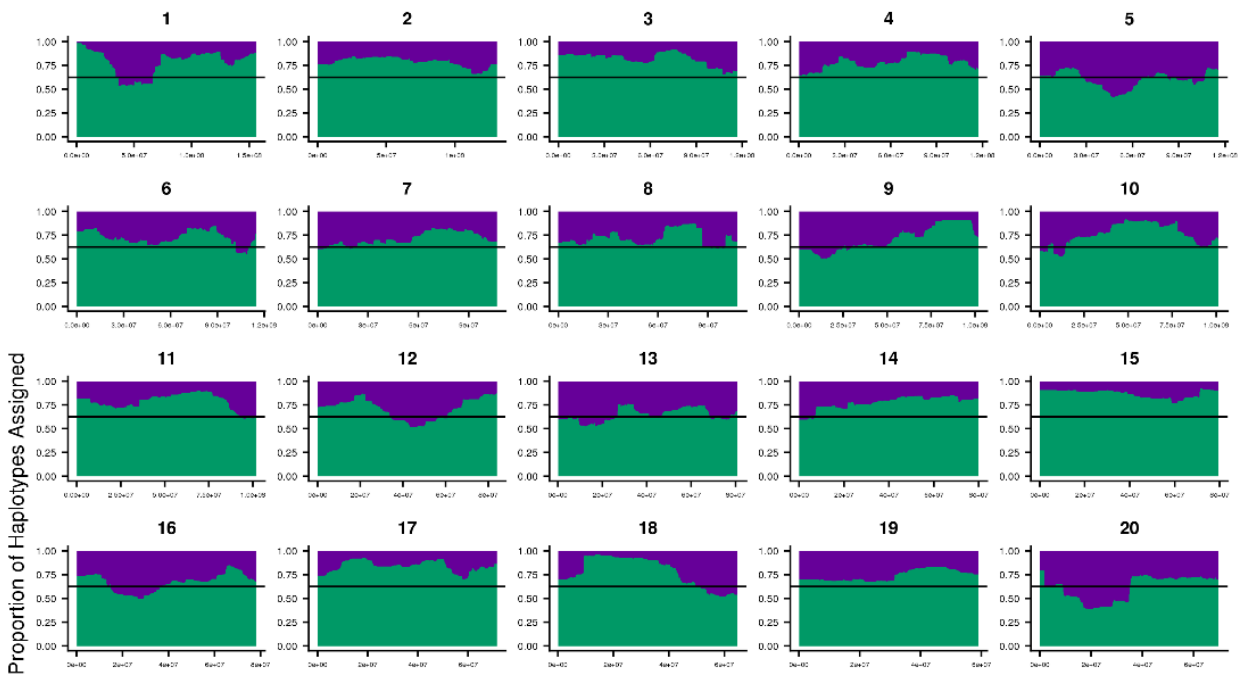

12

13

14

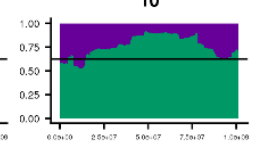

15

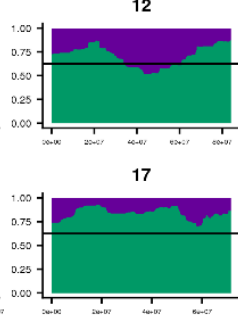

22

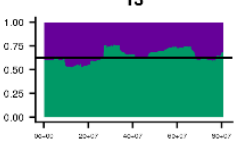

18

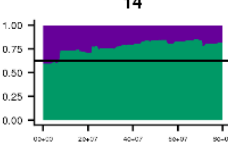

19
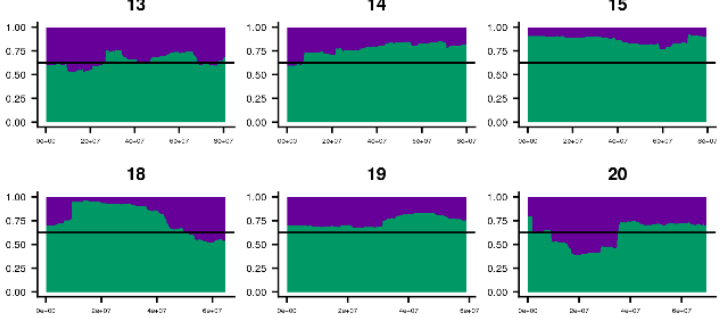

23

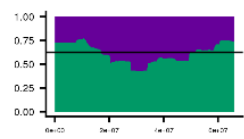

26
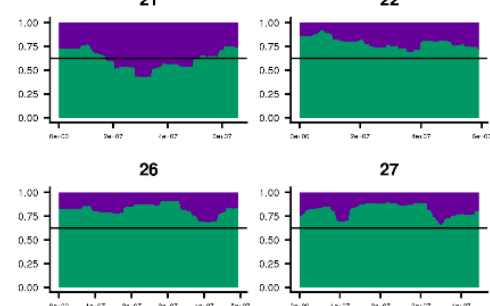

27

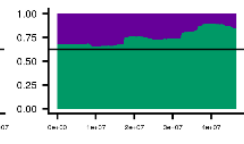

28
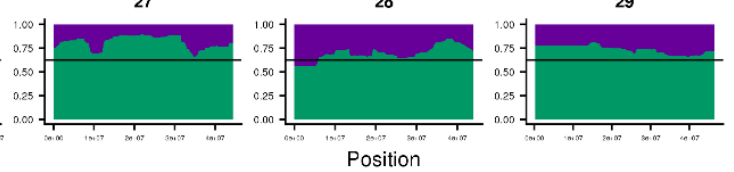

24

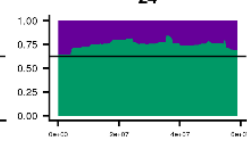

25

20
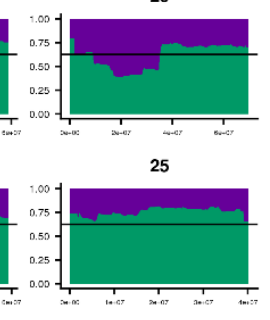

Figure 3.1. Brangus Most Likely Assignment for the EXPANDED Reference. Plot of RFmix most likely assigned ancestry, Brahman (purple) and Angus (green), for each CRF window for Brangus. The horizontal line indicates the $5 / 8$ expected Angus proportion based on pedigree. 

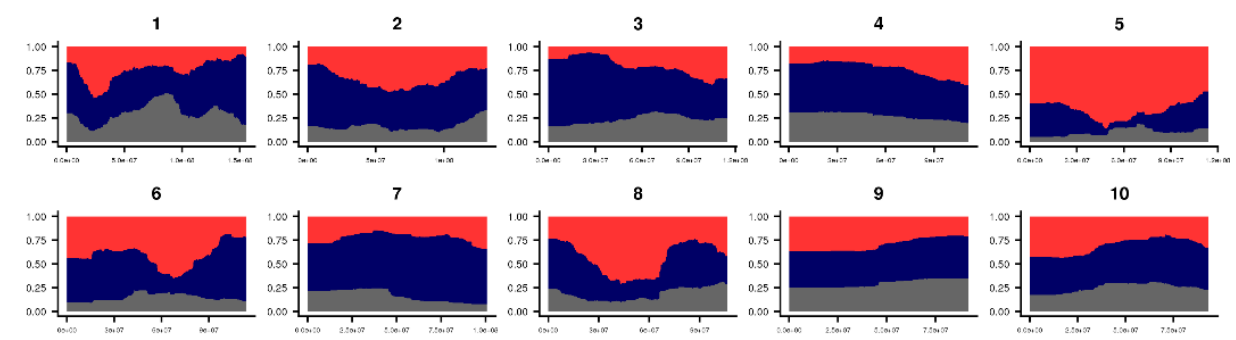

9

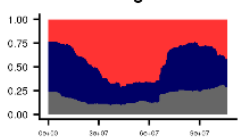

13

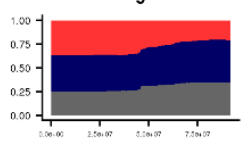

14

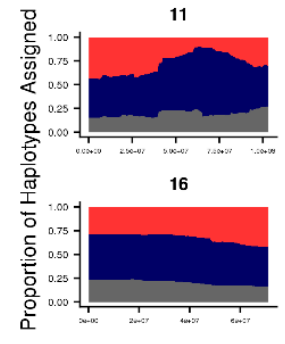

12

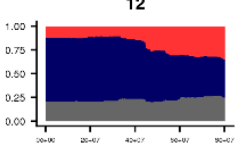

17

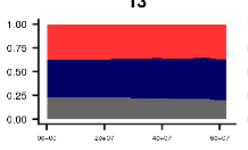

18

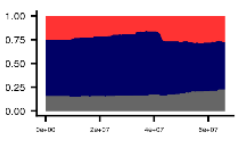

22

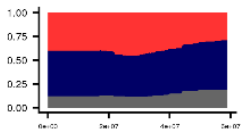

26

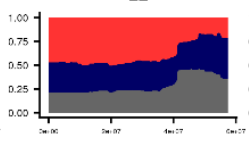

27

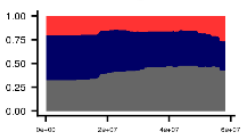

23

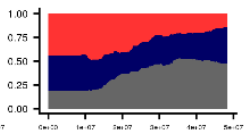

28
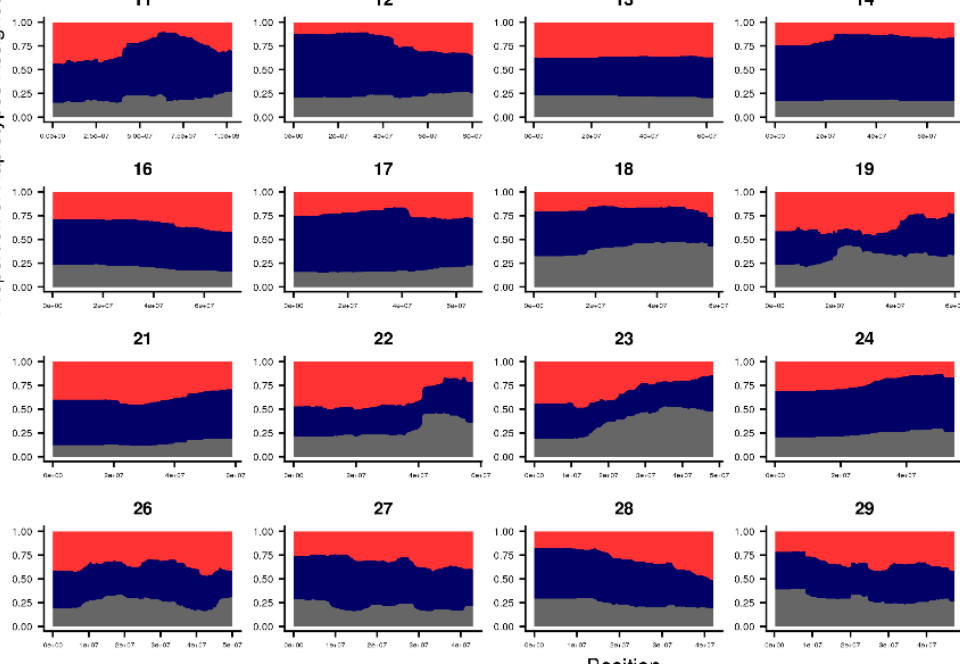

19

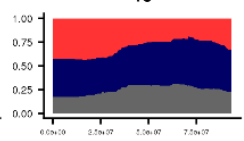

15

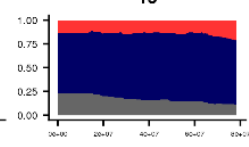

20

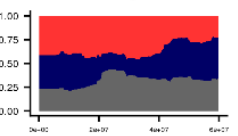

24

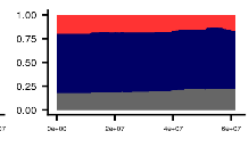

25

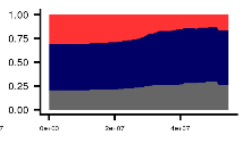

29
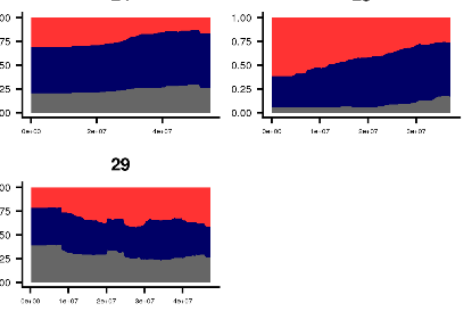

an

Hereford Shorthorn

Figure 3.2. Beefmaster Most Likely Assignment for the EXPANDED Reference.

Plot of RFmix most likely assigned ancestry, Brahman (red), Hereford (navy), and Shorthorn (gray), for each CRF window for Beefmaster. Beefmaster is expected to be $25 \%$ Hereford, $25 \%$ Shorthorn and $50 \%$ Brahman based on pedigree. 

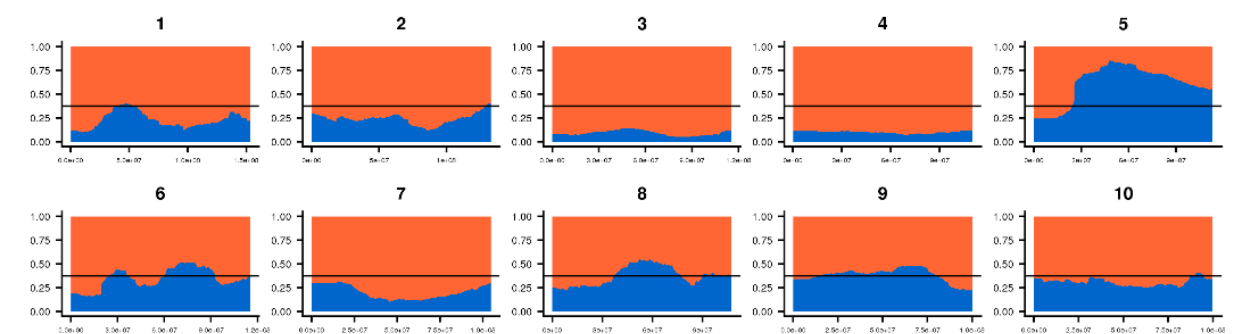

9

10

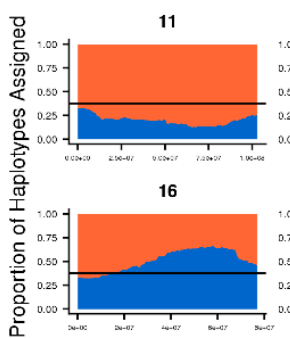

12

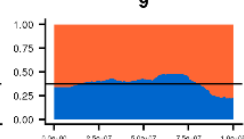

14

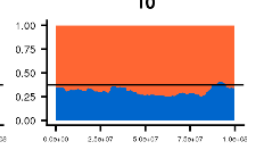

13

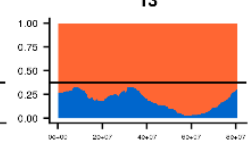

18

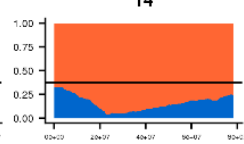

19

17

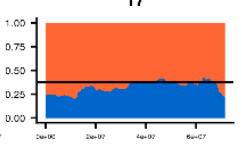

22

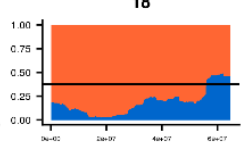

23

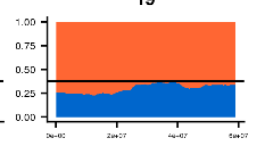

24

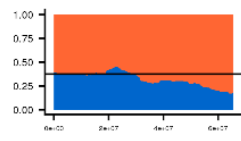

26

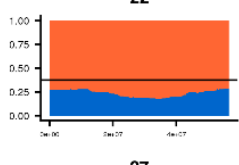

27

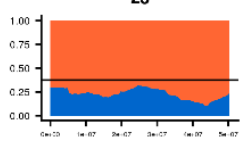

28
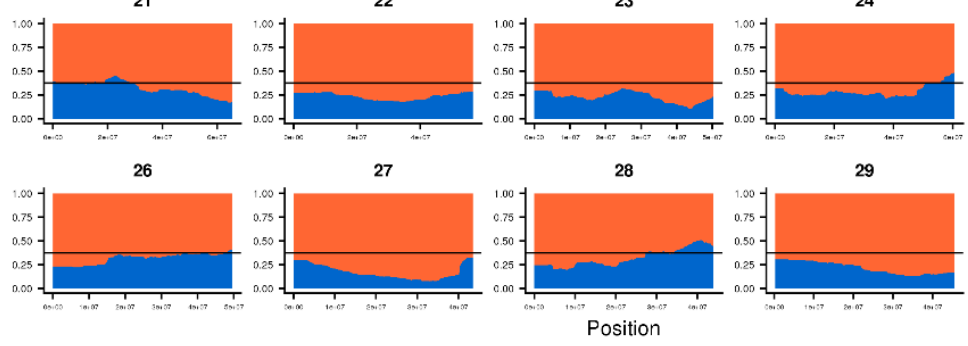

29
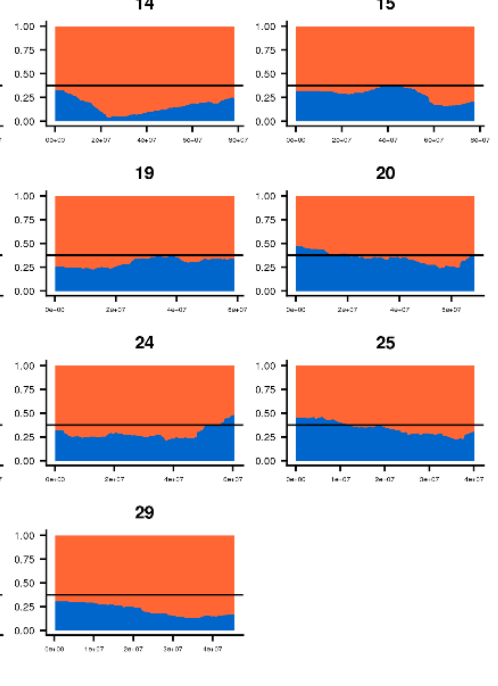

20

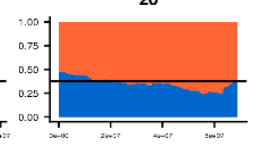

25

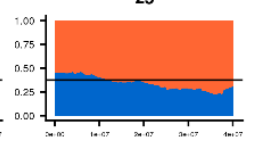

Breed $\square$ Shorthorn $\square$ Brahman

Figure 3.3. Santa Gertrudis Most Likely Assignment for the EXPANDED Reference. Plot of RFmix most likely assigned ancestry, Shorthorn (orange) and Brahman (blue), for each CRF window for Santa Gertrudis. The horizontal line indicates the $3 / 8$ expected Brahman proportion based on pedigree. 

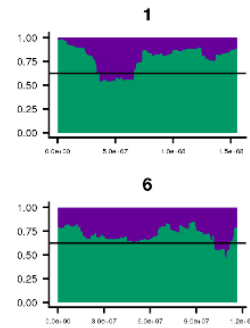

11

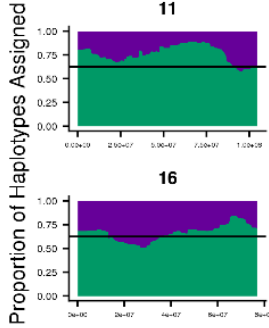

21

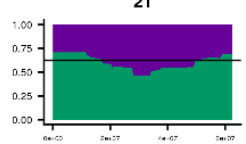

26

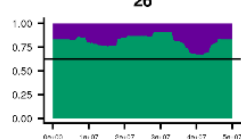

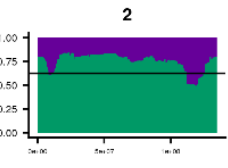

7

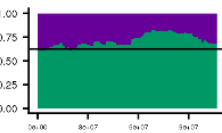

12

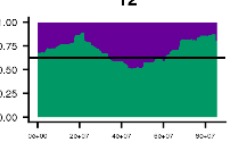

17

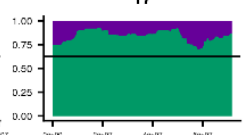

22

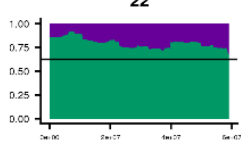

27

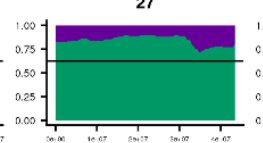

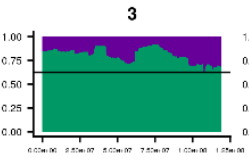

8

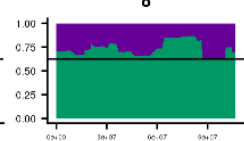

13

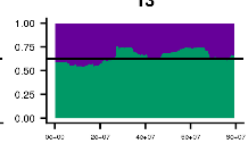

18

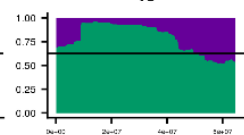

23

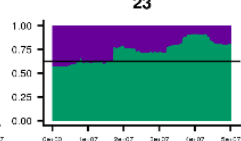

28

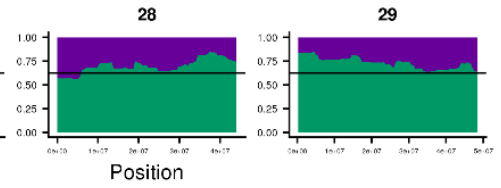

Breed Angus Brahman

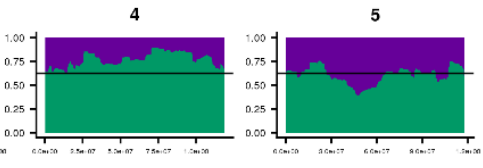

9

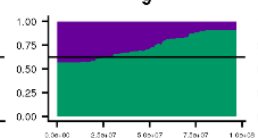

14

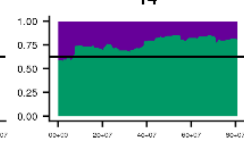

19

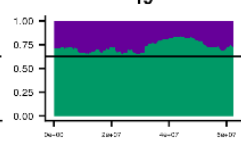

24

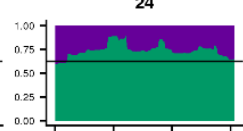

29

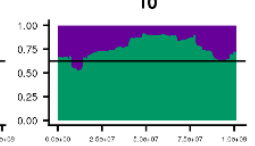

15

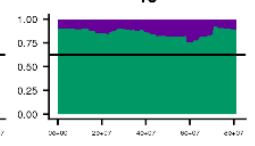

20

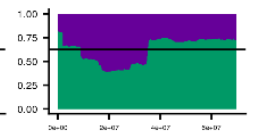

25

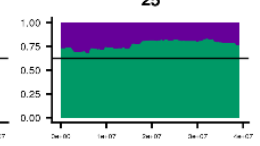

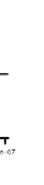

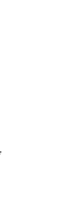

Figure 3.4. Brangus Most Likely Assignment for the CRUMBLER

Reference. Plot of RFmix most likely assigned ancestry, Brahman (purple) and Angus (green), for each CRF window for Brangus. The horizontal line indicates the 5/8 expected Angus proportion based on pedigree. 

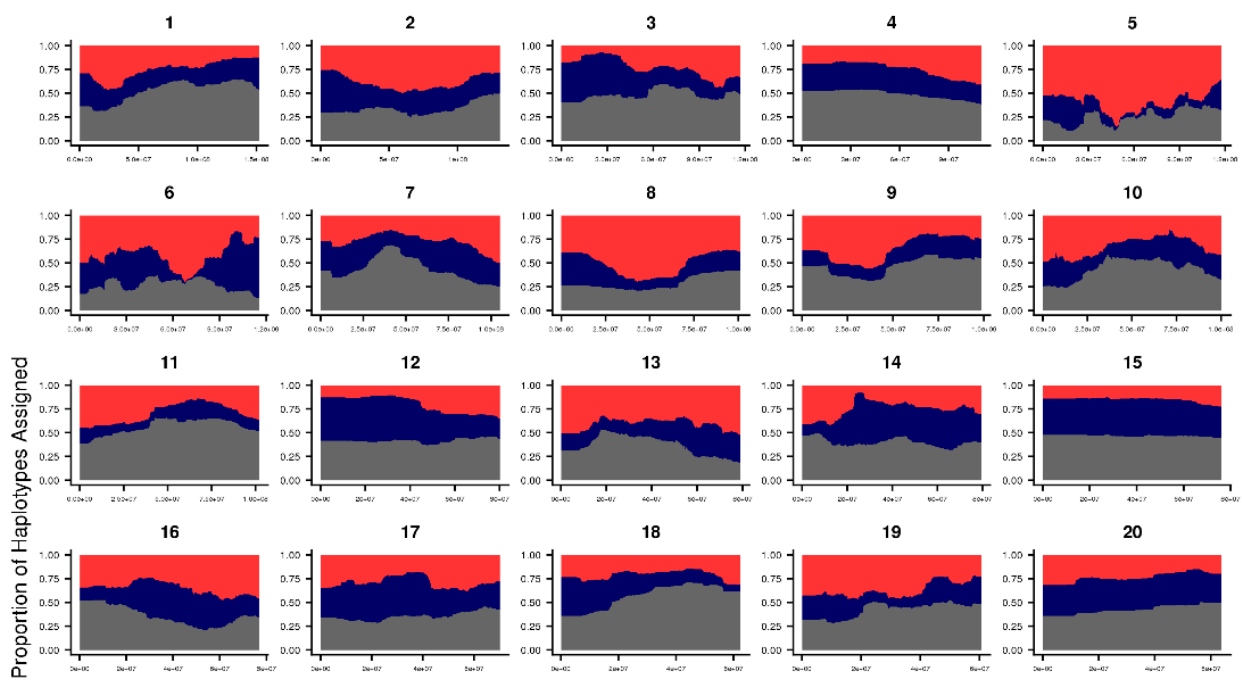

12

13

14

15

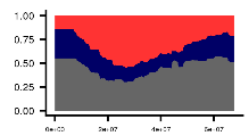

26

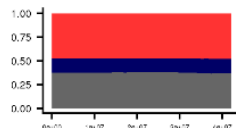

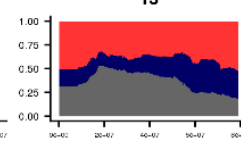

18

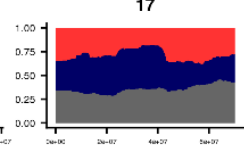

22

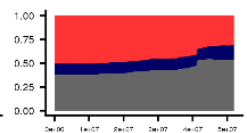

27

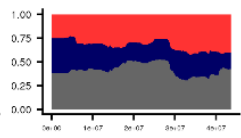

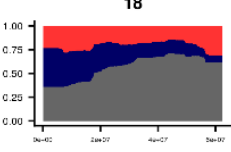

23

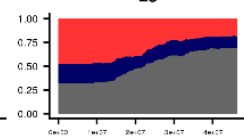

28

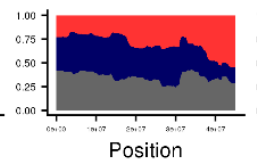

Position

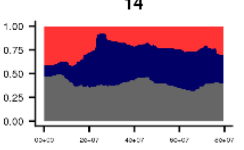

19

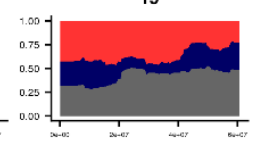

24

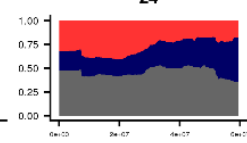

29

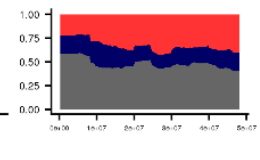

Breed $\square$ Hereford Brahman $\square$ Shorthorn

Figure 3.5. Beefmaster Most Likely Assignment for the CRUMBLER Reference.

Plot of RFmix most likely assigned ancestry, Brahman (red), Hereford (navy), and Shorthorn (gray), for each CRF window for Beefmaster. Beefmaster is expected to be $25 \%$ Hereford, $25 \%$ Shorthorn and $50 \%$ Brahman based on pedigree. 

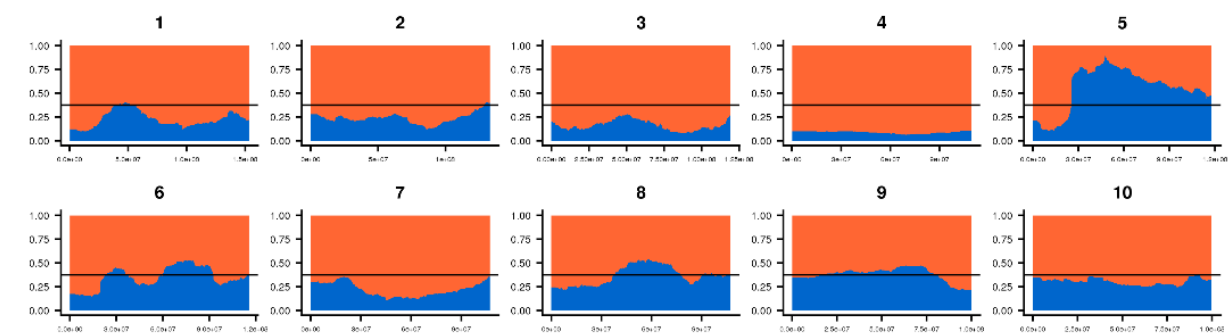

13

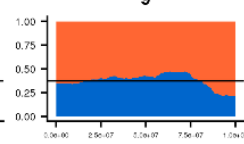

14

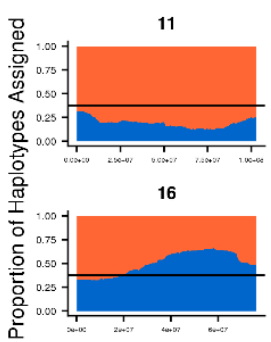

12

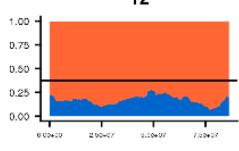

17

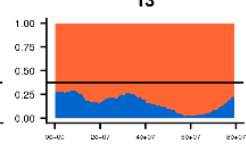

18

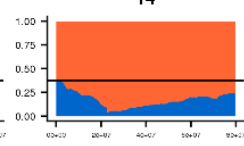

19
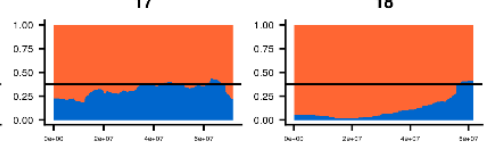

23

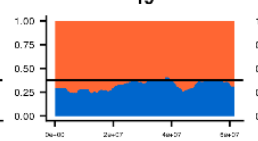

24

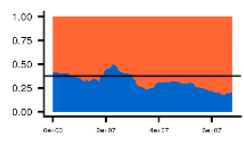

26

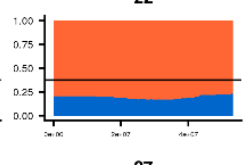

27
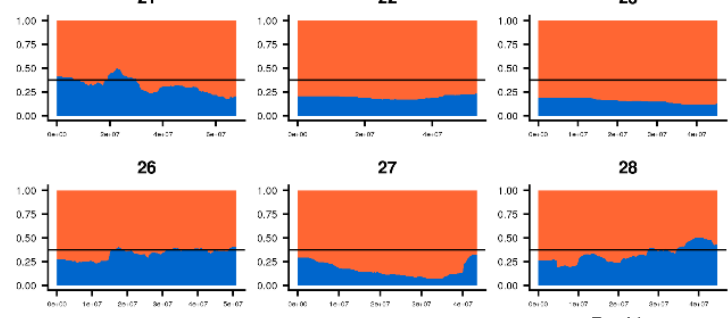

28

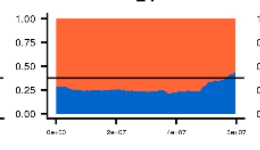

29
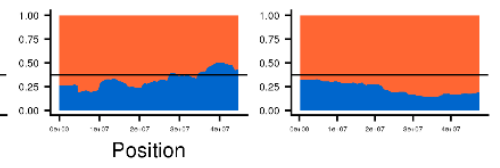

Breed Shorthorn $\square$ Brahman

Figure 3.6. Santa Gertrudis Most Likely Assignment for the

CRUMBLER Reference. Plot of RFmix most likely assigned ancestry, Shorthorn (orange) and Brahman (blue), for each CRF window for Santa Gertrudis. The horizontal line indicates the 3/8 expected Brahman proportion based on pedigree. 
a.
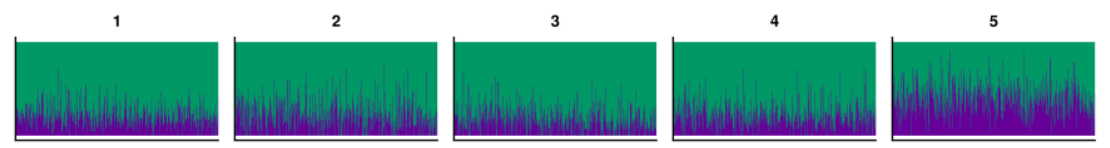

6
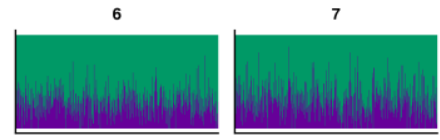

8

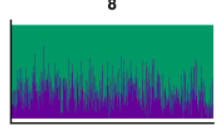

9

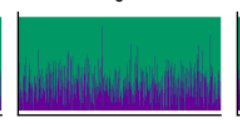

10

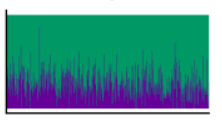

11

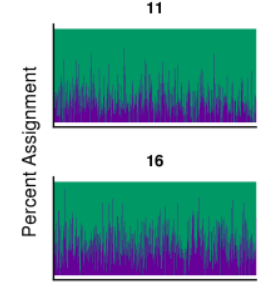

12

13

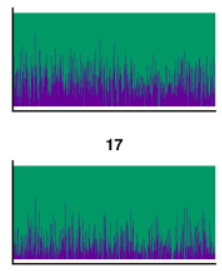

22

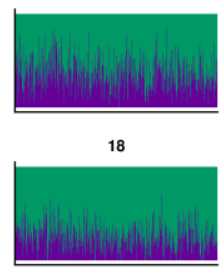

14

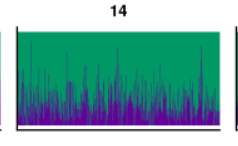

19

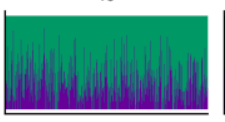

24

23

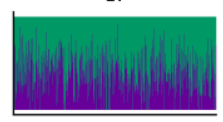

26

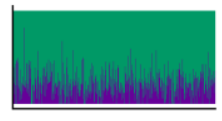

27

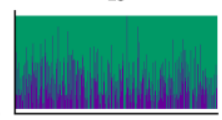

28

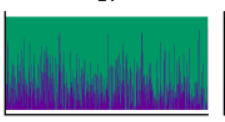

29
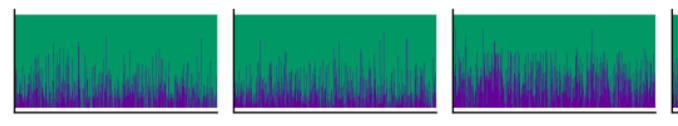

Individual
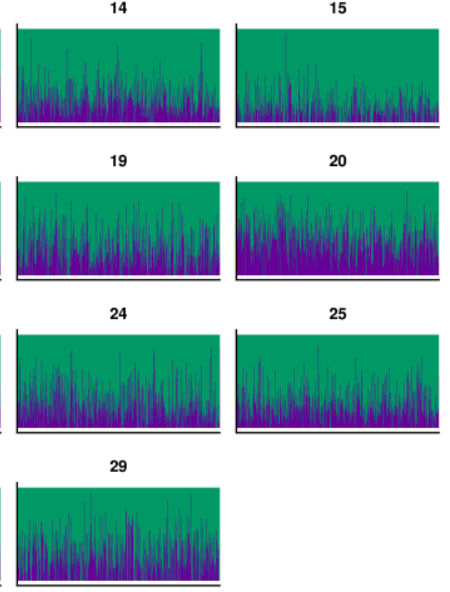

25

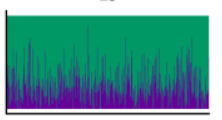

b.
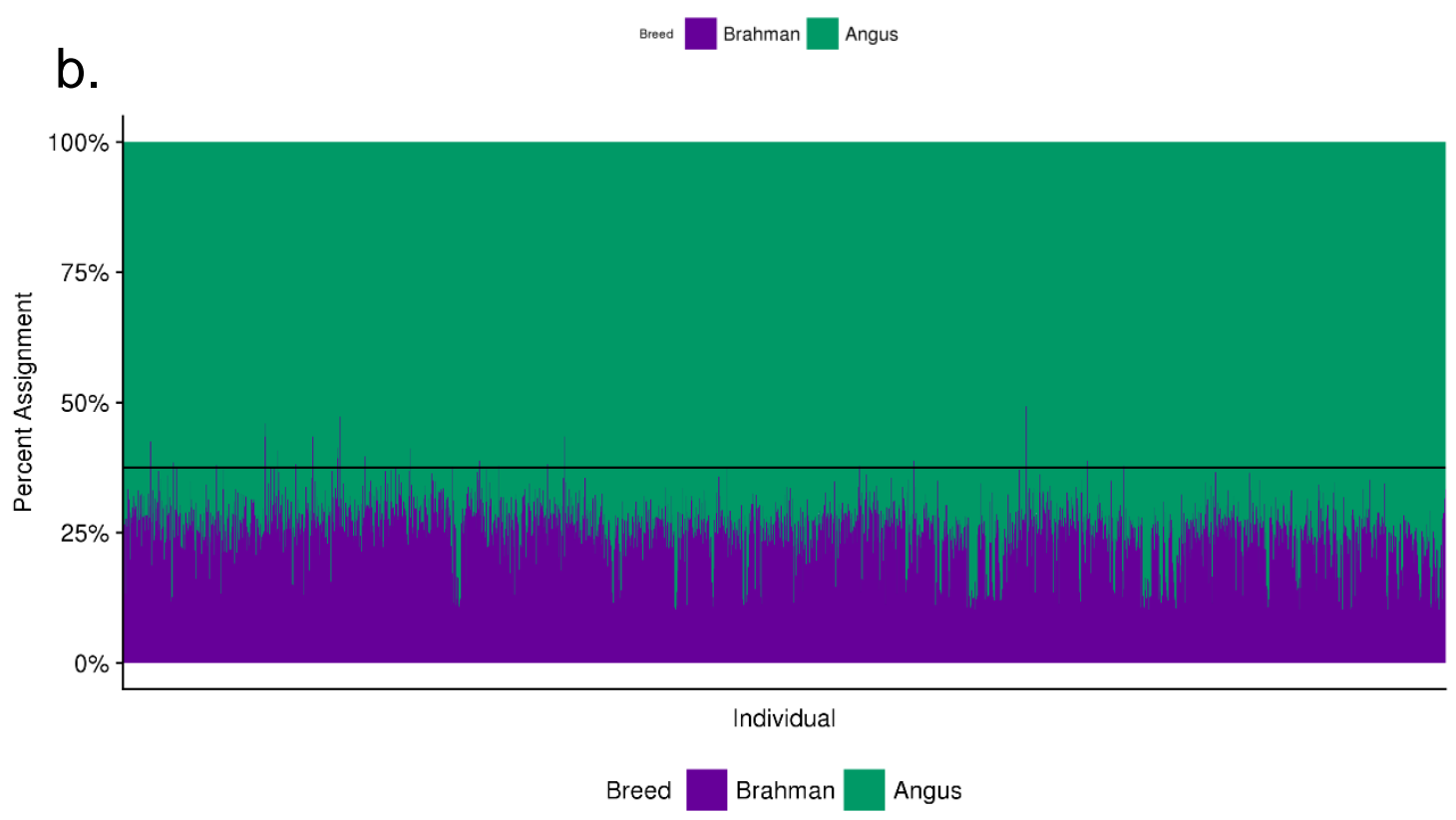

Figure 3.7. Brangus Ancestry Estimation by Individual. (a.) Plot of RFmix global ancestry assignment for Brangus individual for each chromosome. Individuals are represented by vertical bars within each plot. (b.) Plot of RFmix global ancestry assignment for Brangus individuals genome wide. Individuals are represented by vertical bars within each plot. The black line represents the proportion of Brahman (3/8) expected based on foundation principles of the Brangus breed. 
a.
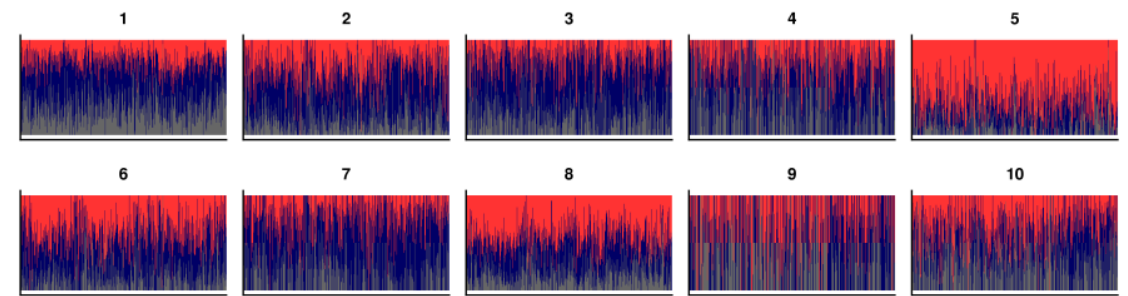

8

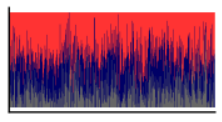

9

10

11

12
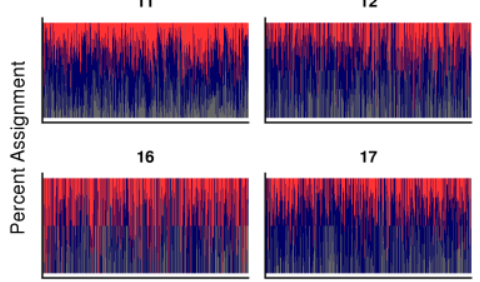

17

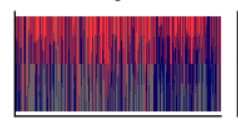

14

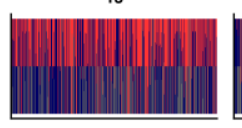

18

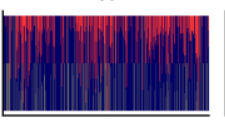

19

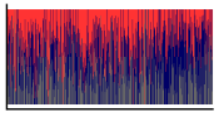

15

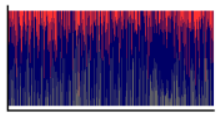

20

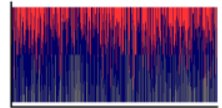

22

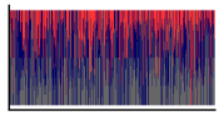

23

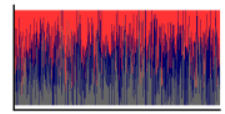

24

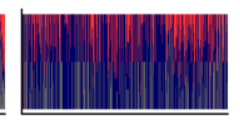

25
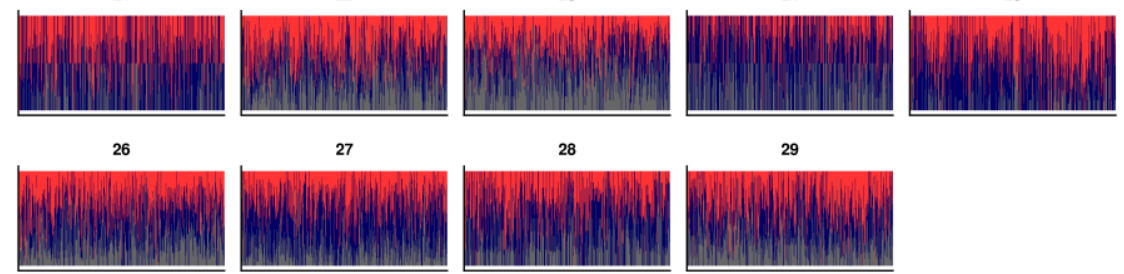

27

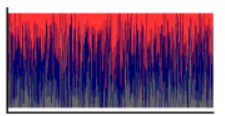

28
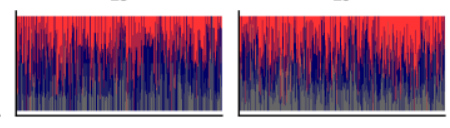

Individual

Breed Brahman $\square$ Hereford Shorthorn

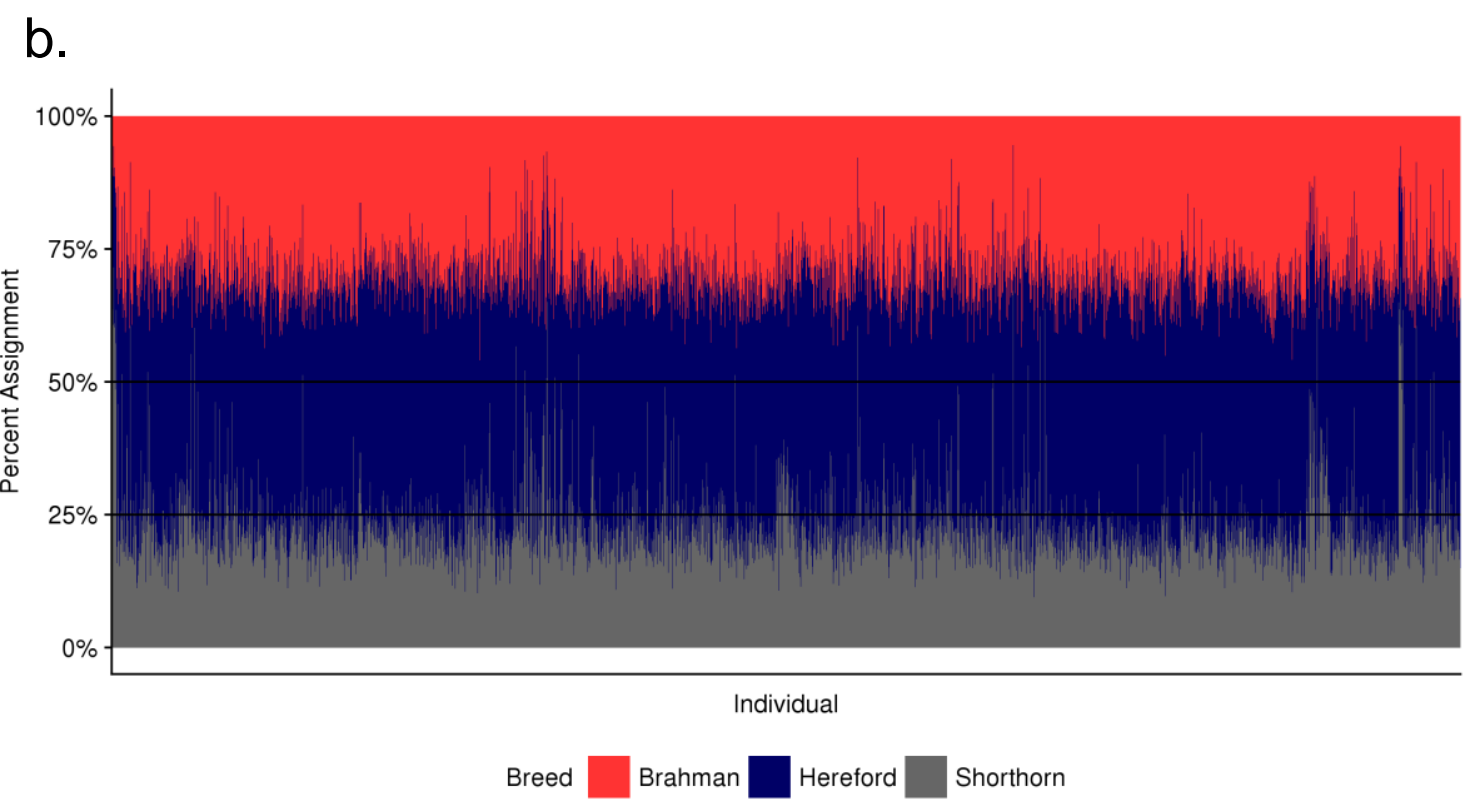

Figure 3.8. Beefmaster Ancestry Estimation by Individual. (a.) Plot of RFmix global ancestry assignment for Brangus individual for each chromosome. Individuals are represented by vertical bars within each plot. (b.) Plot of RFmix global ancestry assignment for Beefmaster individuals genome wide. Individuals are represented by vertical bars within each plot. The black line represents the proportion of Shorthorn (1/4) and Hereford (additional 1/4) expected based on foundation principles of the Beefmaster breed. 
a.

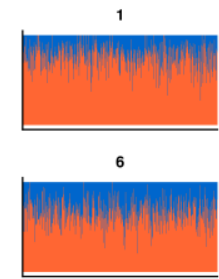

11

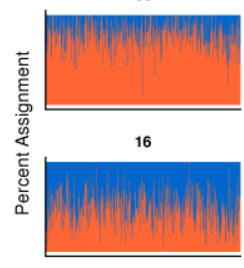

21

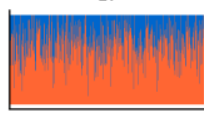

26

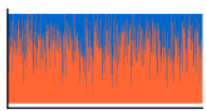

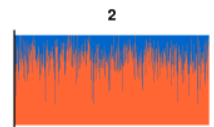

7

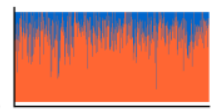

12

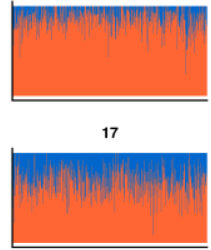

22

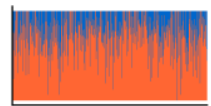

27

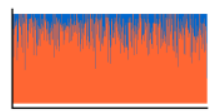

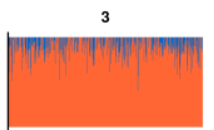

8

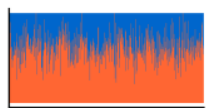

13

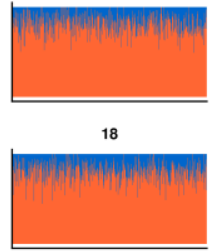

23

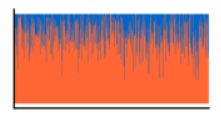

28

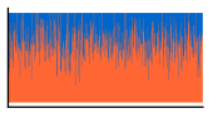

Individual
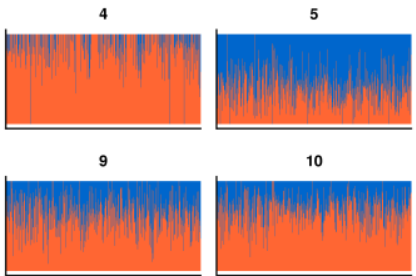

14

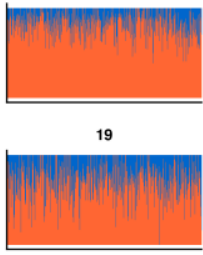

24

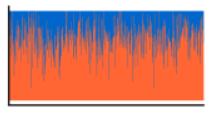

29
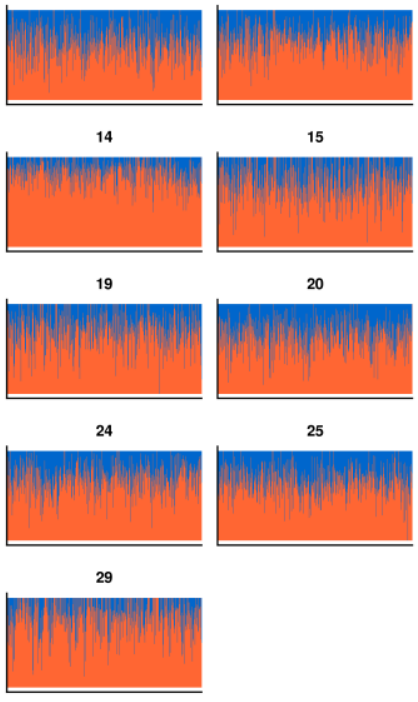

15

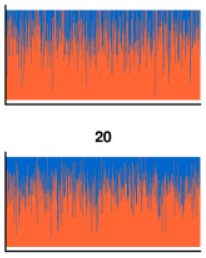

25

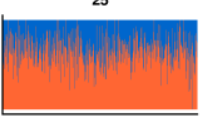

Breed $\square$ Brahman $\square$ Shorthorn

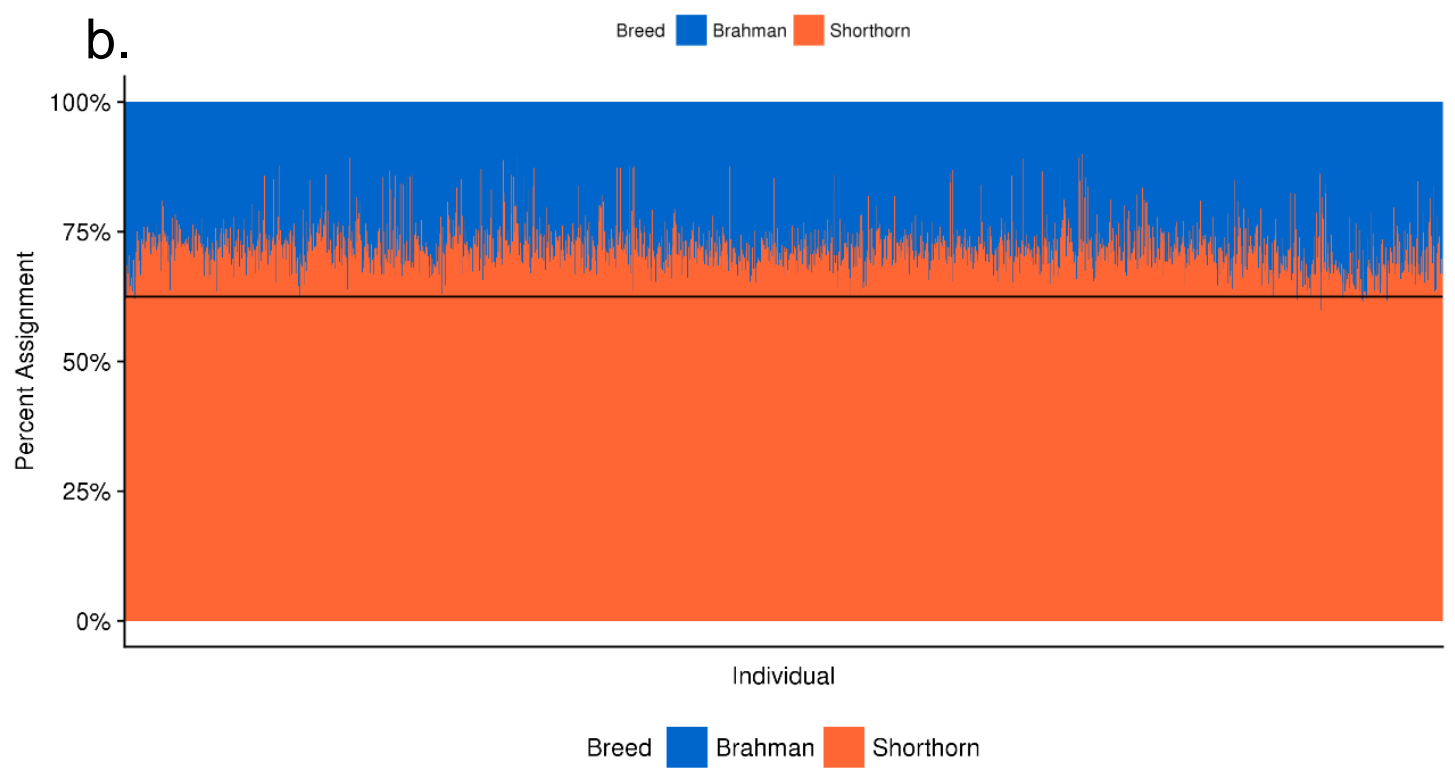

Figure 3.9. Santa Gertrudis Ancestry Estimation by Individual. (a.) Plot of RFmix global ancestry assignment for Santa Gertrudis individual for each chromosome. Individuals are represented by vertical bars within each plot. (b.) Plot of RFmix global ancestry assignment for Santa Gertrudis individuals genome wide. Individuals are represented by vertical bars within each plot. The black line represents the proportion of Shorthorn (5/8) expected based on foundation principles of the Santa Gertrudis breed. 


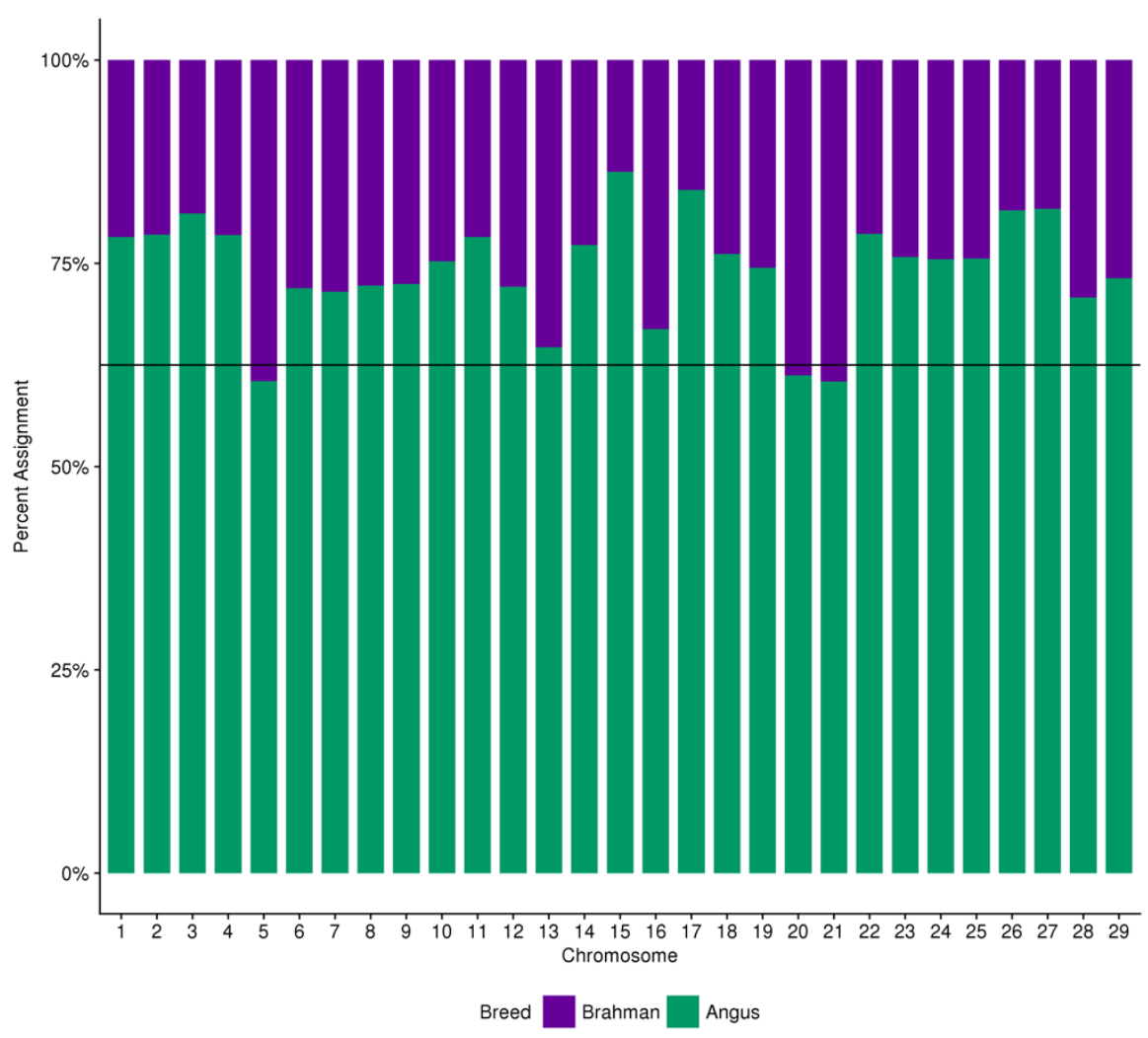

Figure 3.10. Estimated Brangus Ancestry by Chromosome. Plot of RFmix average most likely assignment proportions for each chromosome (chromosome averages depicted here are based upon regions indicated in Figure 3.1). The black line represents the expected Angus proportion (5/8) based on pedigree. 


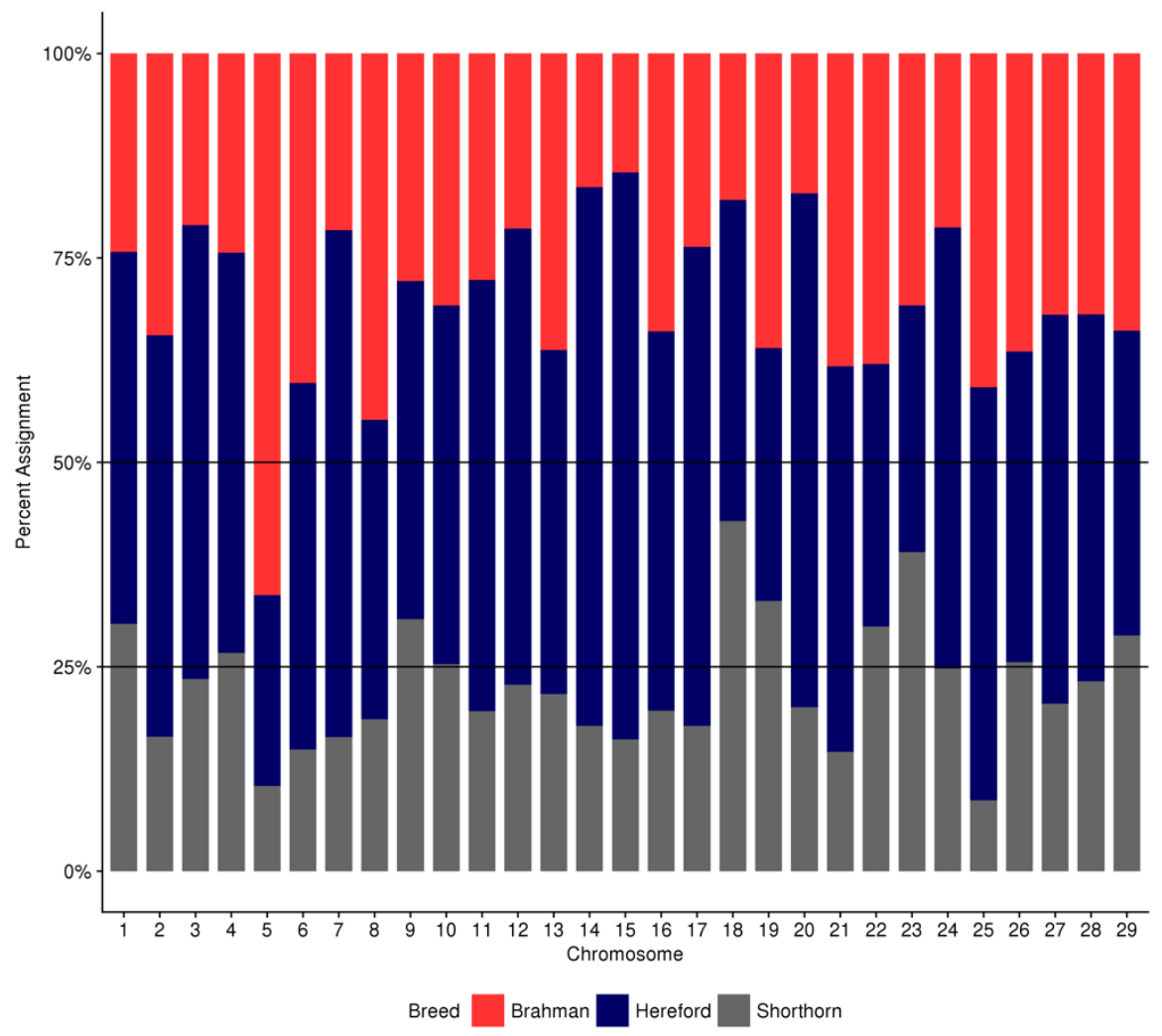

Figure 3.11. Estimated Beefmaster Ancestry by Chromosome. Plot of RFmix average most likely assignment proportions for each chromosome (chromosome averages depicted here are based upon regions indicated in Figure 3.2). The black line represents the expected Shorthorn (1/4) and Hereford proportions (additional 1/4) based on pedigree. 


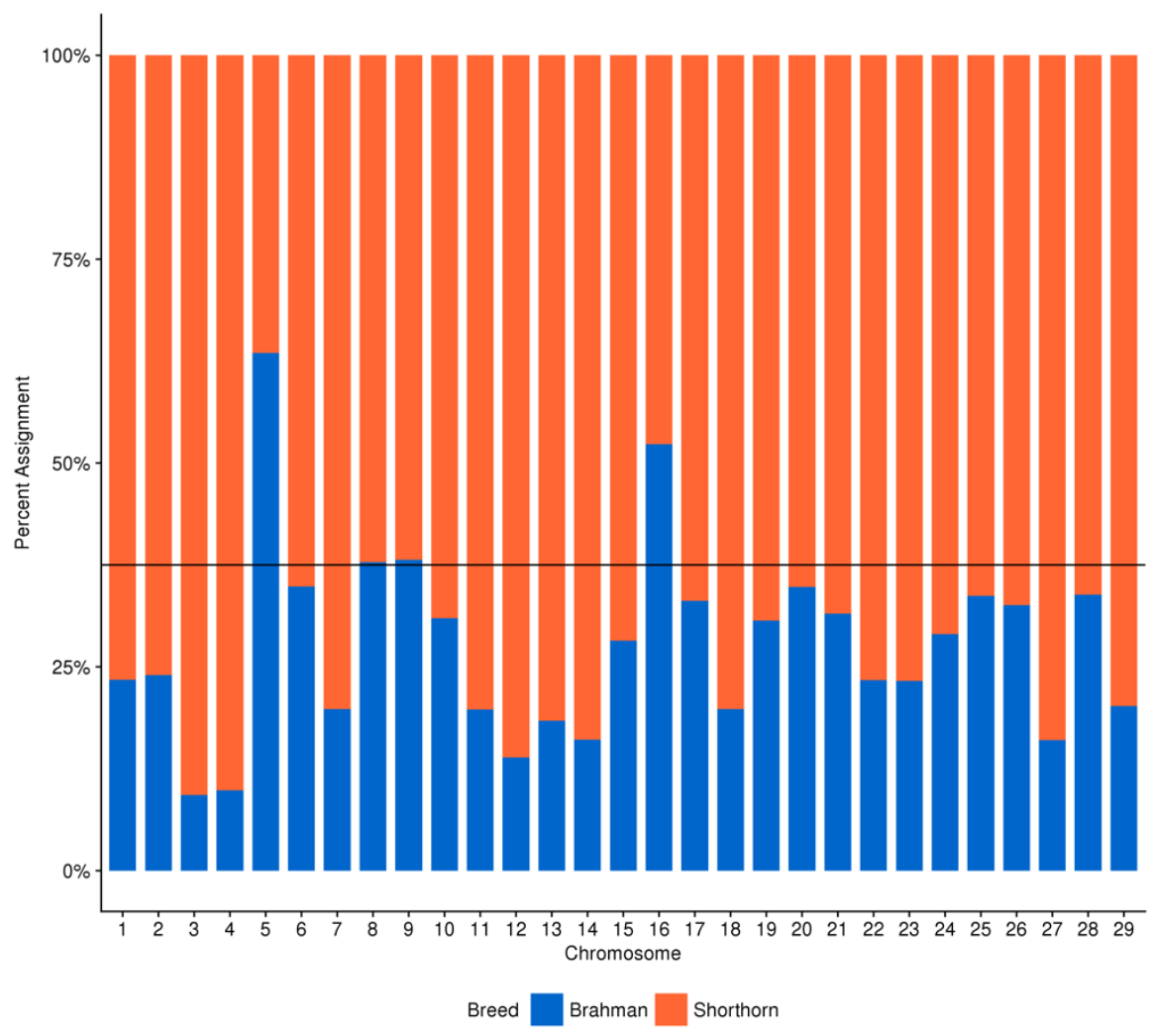

Figure 3.12. Estimated Santa Gertrudis Ancestry by Chromosome. Plot of RFmix average most likely assignment proportions for each chromosome (chromosome averages depicted here are based upon regions indicated in Figure 3.3). The black line represents the expected Brahman proportion (3/8) based on pedigree. 
a.
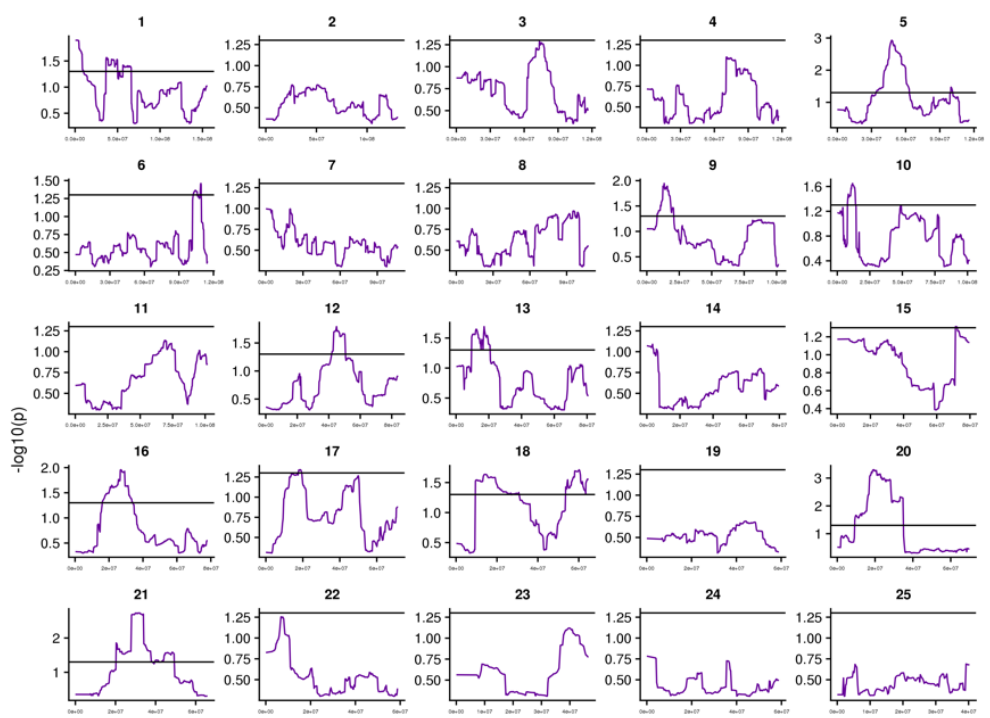

$23 \quad 24$
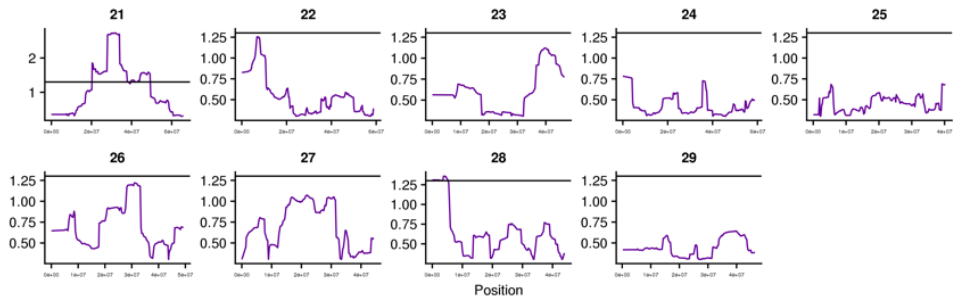

b.
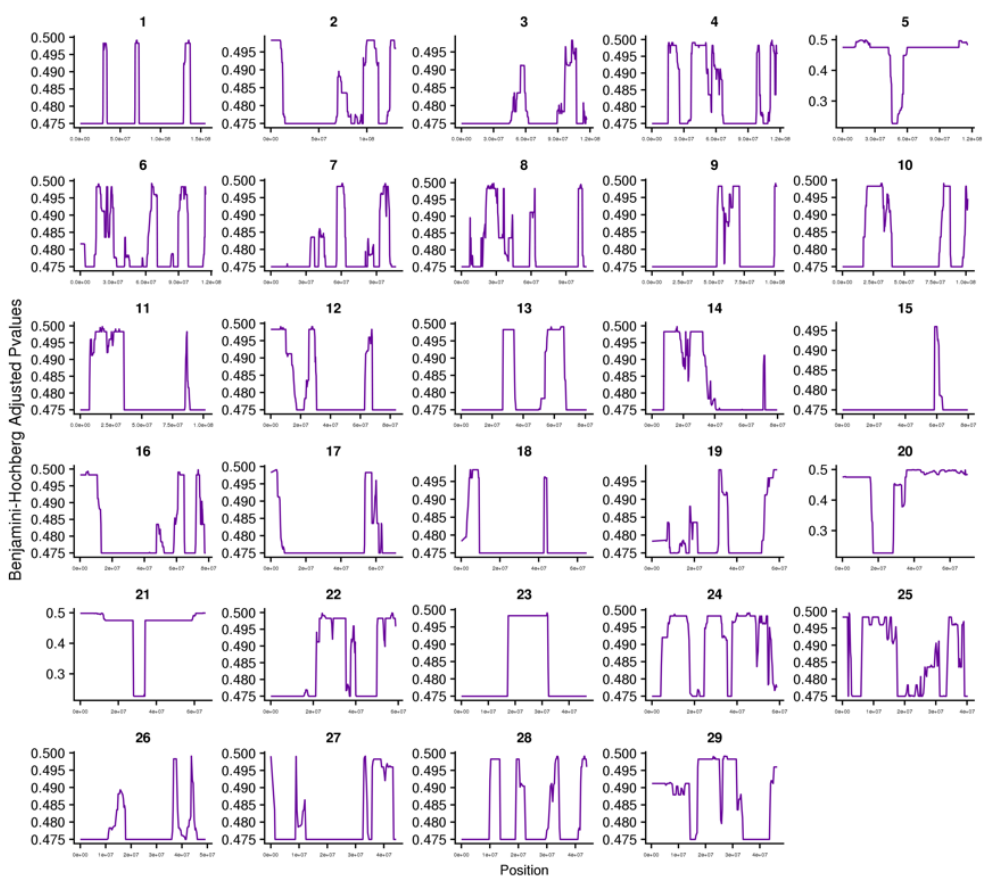

Figure 3.13. Significant Regions of Deviation from Genome-Wide Brahman Proportion for Brangus by Chromosome. (a). Plot of - $\log 10 \mathrm{p}$ values indicating regions of the Brangus genome that deviate from the genome-wide average Brahman proportion (25.68\%). The black line indicates the $p$-value $=0.05$ significance threshold. (b). Corresponding plot using Benjamini-Hochberg adjusted pvalues to correct for multiple testing and false positive errors. 
a.
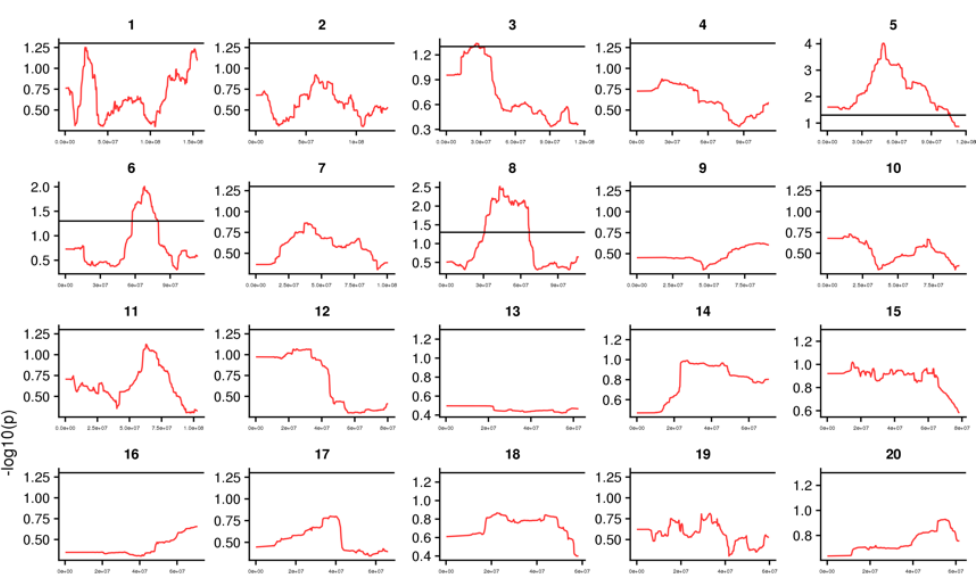

13
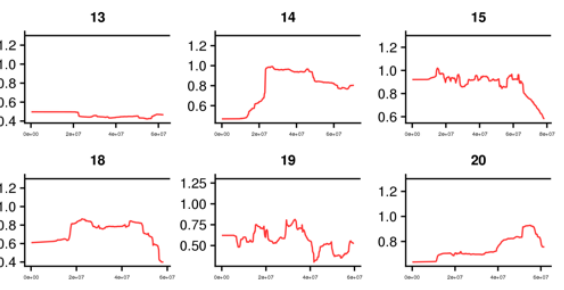

2
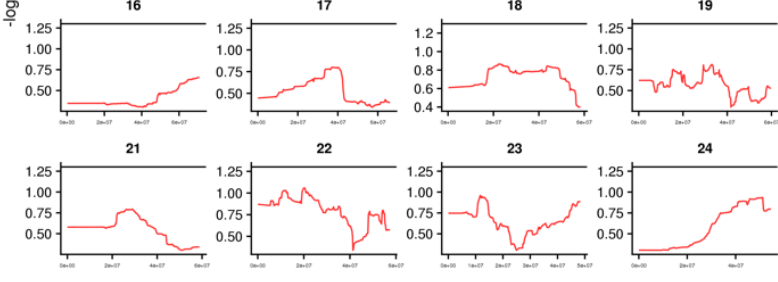

23

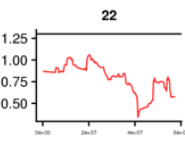

24
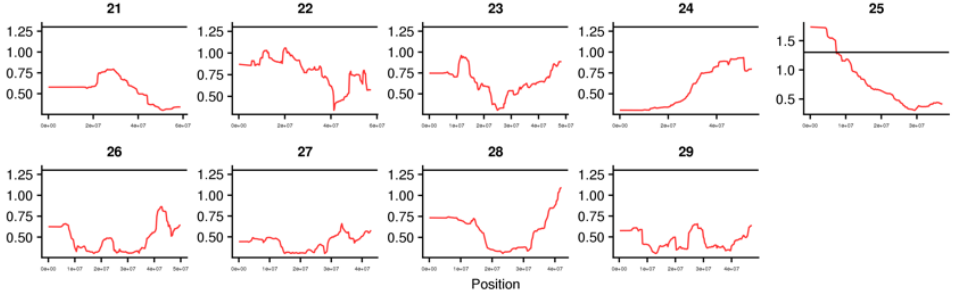

b.
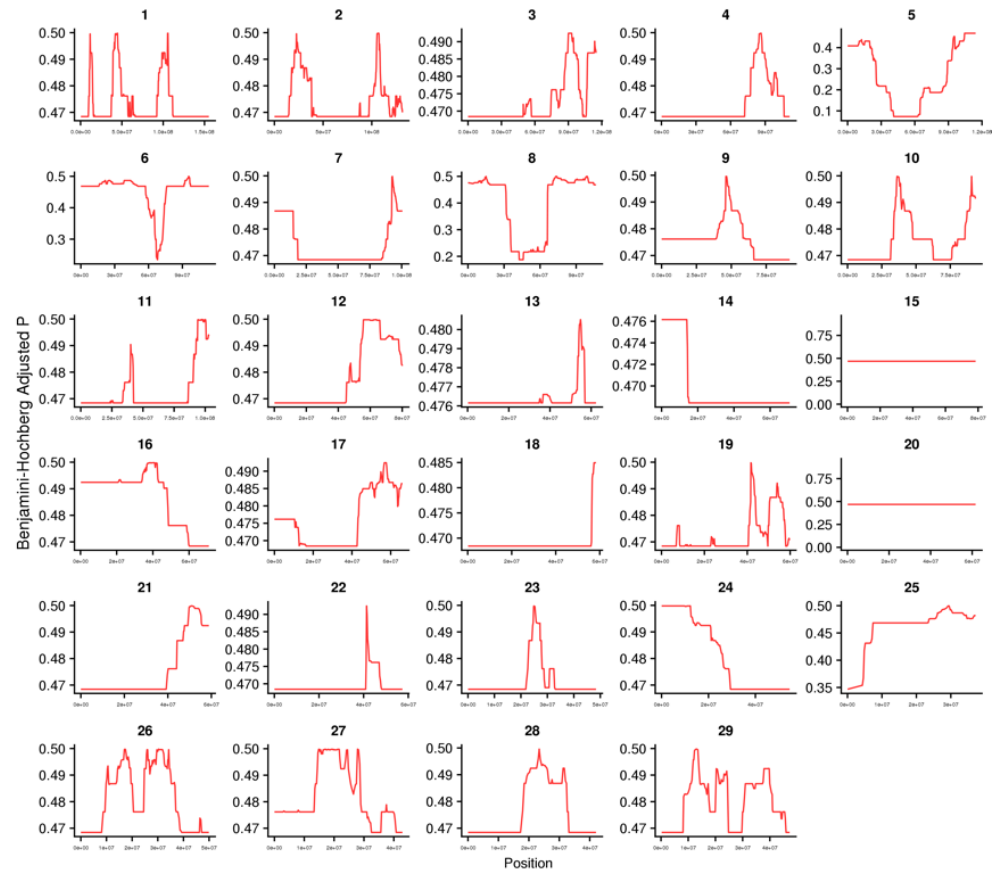

Figure 3.14. Significant Regions of Deviation from Genome-Wide Brahman Proportion for Beefmaster by Chromosome. (a). Plot of $-\log 10 \mathrm{p}$ values indicating regions of the Beefmaster genome that deviate from the genome-wide average Brahman proportion (30.84\%). The black line indicates the $p$-value $=0.05$ significance threshold. (b). Corresponding plot using Benjamini-Hochberg adjusted pvalues to correct for multiple testing and false positive errors. 
a.
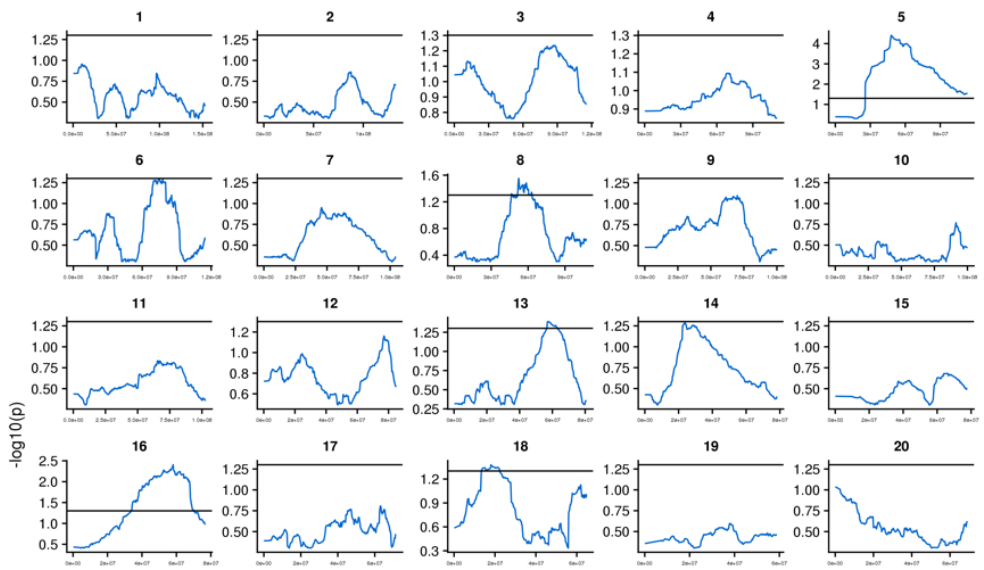

15
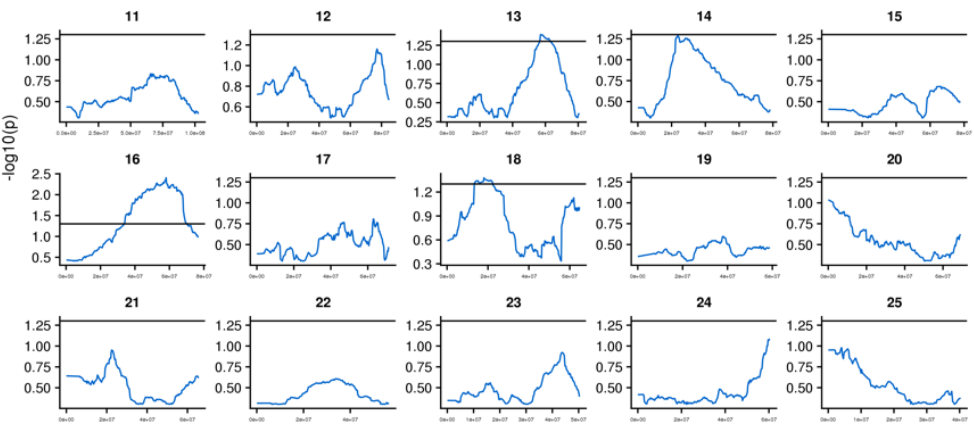

22
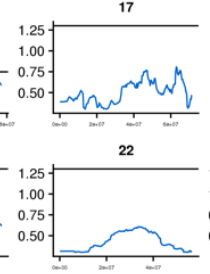

23
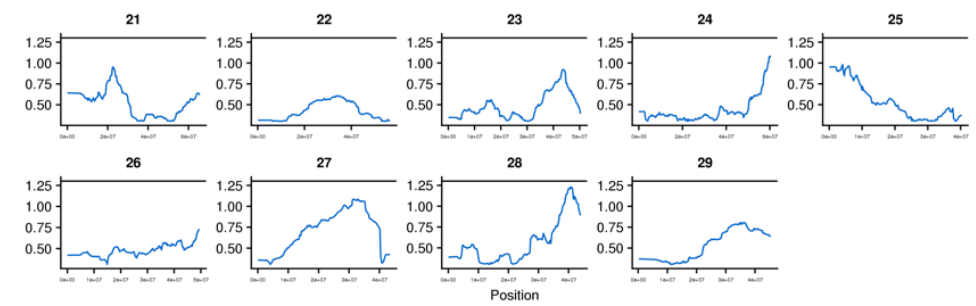

b.
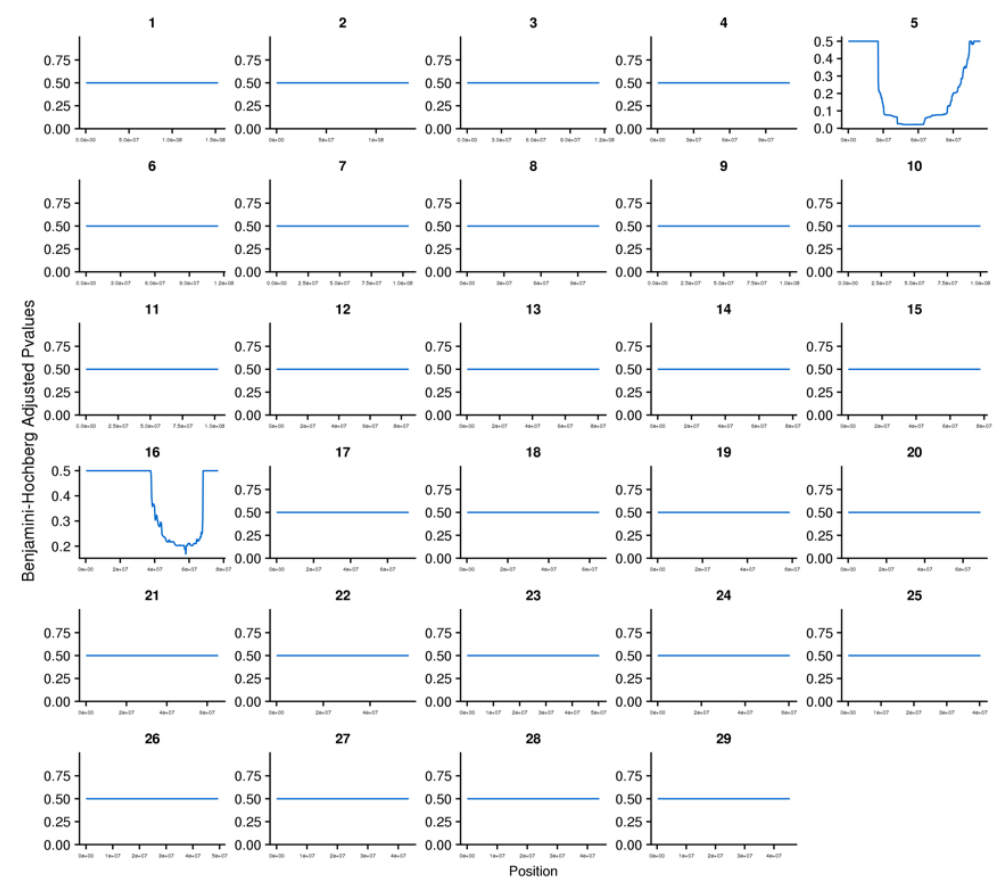

Figure 3.15. Significant Regions of Deviation from Genome-Wide Brahman Proportion for Santa Gertrudis by Chromosome. (a). Plot of $\log 10 p$-values indicating regions of the Santa Gertrudis genome that deviate from the genome-wide average Brahman proportion (27.81\%). The black line indicates the $p$ value $=0.05$ significance threshold. (b). Corresponding plot using Benjamini-Hochberg adjusted $\mathrm{p}$-values to correct for multiple testing and false positive errors. 


\section{Tables}

Table 3.1. Genotype data for American breeds.

\begin{tabular}{cccc} 
Assay $^{\mathrm{a}}$ & $\begin{array}{c}\text { No. } \\
\text { Brangus }\end{array}$ & $\begin{array}{c}\text { No. } \\
\text { Beefmaster }\end{array}$ & $\begin{array}{c}\text { No. Santa } \\
\text { Gertrudis }\end{array}$ \\
\hline BOVG50v1 & 0 & 836 & 264 \\
GGP90KT & 688 & 0 & 6 \\
GGPHDV3 & 1,003 & 1,199 & 0 \\
GGPLDV3 & 0 & 36 & 756 \\
GGPLDV4 & 5,597 & 304 & 897 \\
HD & 982 & 0 & 23 \\
BovineSNP50 & 1,174 & 65 & 0 \\
Zoetis i50K & 14 & 1,332 & 0 \\
Total & 9,458 & 3,772 & 1,946 \\
Total $^{\mathrm{c}}$ & 9,161 & 3,762 & 1,942 \\
\hline
\end{tabular}

a Genotyping platforms used to genotype the samples. Genotypes for all individuals were imputed to 836,118 genotypes.

${ }^{b}$ Number of American breed individuals remaining following quality control after imputation and phasing.

c Number of American breed individuals remaining following filtering for nonadvanced generation composite samples. 
Table 3.2. Genotype data for individuals from 5 breeds used to generate the reference population.

\begin{tabular}{|c|c|c|c|c|c|c|}
\hline Breed & $\begin{array}{c}\text { No. } \\
\text { Registered }^{\mathrm{a}}\end{array}$ & $\begin{array}{c}\text { No. } \\
\text { Individuals } \\
>85 \%\end{array}$ & $\begin{array}{c}\text { CRUMBLER } \\
\text { Reference } \\
\text { Panel }^{c}\end{array}$ & $\begin{array}{l}\text { Expanded } \\
\text { Reference: }^{\text {Brangus }}{ }^{d}\end{array}$ & $\begin{array}{c}\text { Expanded } \\
\text { Reference: } \\
\text { Santa } \\
\text { Gertrudis }^{d}\end{array}$ & $\begin{array}{l}\text { Expanded } \\
\text { Reference: } \\
\text { Beefmaster }\end{array}$ \\
\hline Angus & 6,699 & 252 & 200 & $997^{e}$ & - & - \\
\hline Hereford & 3,651 & 227 & 200 & - & - & 500 \\
\hline Shorthorn & 487 & 183 & 183 & - & 487 & 487 \\
\hline Brahman & 954 & 361 & 200 & 954 & 500 & 500 \\
\hline Total & 11,791 & 1,422 & 783 & 1,954 & 987 & 1,487 \\
\hline
\end{tabular}

a Total number of registered samples available for analysis.

b Number of registered animals identified with $\geq 85 \%$ assignment probability to the respective breed using the CRUMBLER pipeline.

${ }^{c}$ A random sample of up to 200 animals per breed from individuals that were estimated by CRUMBLER with $\geq 85 \%$ assignment probability to their respective breed.

${ }^{d}$ Of the ancestral breeds used to develop each American breed, that with the fewest registered animals determined the approximate sample size for the remaining breeds, from which random samples of the total number of registered individuals were obtained.

e A random sample of 1000 Angus individuals was taken, of which, 997 animals remained following quality control for imputation and phasing. 
Table 3.3. Proportion of haplotypes by chromosome for each American breed with marginal probabilities of most likely breed assignment by RFmix between 0.05 and 0.95 .

\begin{tabular}{|c|c|c|c|}
\hline Chromosome & Brangus & Beefmaster & Santa Gertrudis \\
\hline 1 & 0.0125 & 0.0325 & 0.0202 \\
\hline 2 & 0.0109 & 0.0234 & 0.0285 \\
\hline 3 & 0.0111 & 0.0193 & 0.0105 \\
\hline 4 & 0.0143 & 0.0147 & 0.0082 \\
\hline 5 & 0.014 & 0.0163 & 0.0217 \\
\hline 6 & 0.0166 & 0.0228 & 0.029 \\
\hline 7 & 0.0186 & 0.0148 & 0.0187 \\
\hline 8 & 0.0152 & 0.0258 & 0.0298 \\
\hline 9 & 0.0149 & 0.0158 & 0.0267 \\
\hline 10 & 0.0209 & 0.0194 & 0.0301 \\
\hline 11 & 0.0110 & 0.0236 & 0.0181 \\
\hline 12 & 0.0164 & 0.0194 & 0.0211 \\
\hline 13 & 0.0211 & 0.0107 & 0.0283 \\
\hline 14 & 0.0177 & 0.0132 & 0.0221 \\
\hline 15 & 0.0076 & 0.0167 & 0.0163 \\
\hline 16 & 0.0210 & 0.0138 & 0.032 \\
\hline 17 & 0.0169 & 0.0232 & 0.0444 \\
\hline 18 & 0.0162 & 0.0183 & 0.0265 \\
\hline 19 & 0.0156 & 0.0334 & 0.0242 \\
\hline 20 & 0.0218 & 0.0133 & 0.0352 \\
\hline 21 & 0.0203 & 0.0147 & 0.0272 \\
\hline 22 & 0.0213 & 0.0294 & 0.0247 \\
\hline 23 & 0.0150 & 0.0282 & 0.0345 \\
\hline 24 & 0.0172 & 0.0221 & 0.0377 \\
\hline
\end{tabular}




\begin{tabular}{cccc}
25 & 0.0254 & 0.0231 & 0.0388 \\
26 & 0.0130 & 0.0437 & 0.038 \\
27 & 0.0306 & 0.0481 & 0.0331 \\
28 & 0.0228 & 0.0271 & 0.043 \\
29 & 0.0133 & 0.0374 & 0.0157 \\
\hline
\end{tabular}


Table 3.4. Average ancestry to reference populations throughout the genomes for each of the American breeds*.

\begin{tabular}{|c|c|c|c|c|}
\hline Chr & $\begin{array}{l}\text { Av. \% Brahman } \\
\text { Brangus } \\
\text { (S.D.) }\end{array}$ & $\begin{array}{c}\text { Av. \% Brahman } \\
\text { Santa Gertrudis } \\
\text { (S.D.) }\end{array}$ & $\begin{array}{l}\text { Av. \% Brahman } \\
\text { Beefmaster } \\
\text { (S.D.) }\end{array}$ & $\begin{array}{l}\text { Av. \% Hereford } \\
\text { Beefmaster } \\
\text { (S.D.) }\end{array}$ \\
\hline 1 & 21.77 (12.65) & 23.41 (8.32) & $24.26(11.27)$ & $45.50(10.85)$ \\
\hline 2 & $21.45(5.14)$ & $24.01(5.96)$ & $34.46(9.01)$ & $49.11(7.50)$ \\
\hline 3 & $18.86(6.92)$ & $9.27(2.77)$ & 21.01 (10.37) & $55.50(13.06)$ \\
\hline 4 & $21.50(6.63)$ & $9.85(1.24)$ & $24.36(8.30)$ & $48.93(4.72)$ \\
\hline 5 & $39.48(8.50)$ & $63.52(16.80)$ & $66.25(9.37)$ & $23.32(10.34)$ \\
\hline 6 & $28.06(7.54)$ & $34.85(10.36)$ & $40.29(12.96)$ & $44.82(15.15)$ \\
\hline 7 & $28.49(6.50)$ & $19.80(7.04)$ & $21.61(4.76)$ & $62.01(5.80)$ \\
\hline 8 & $27.71(7.97)$ & $37.84(10.09)$ & $44.80(17.04)$ & $36.63(12.54)$ \\
\hline 9 & $27.54(13.15)$ & $38.12(7.78)$ & $27.84(6.52)$ & $41.33(2.72)$ \\
\hline 10 & 24.75 (11.23) & $30.98(4.00)$ & $30.82(8.16)$ & $43.86(4.68)$ \\
\hline 11 & $21.76(8.55)$ & $19.75(4.96)$ & 27.68 (11.09) & $52.73(10.19)$ \\
\hline 12 & 27.87 (10.99) & $13.87(3.78)$ & 21.40 (9.32) & $55.82(10.89)$ \\
\hline 13 & $35.34(6.93)$ & $18.38(9.32)$ & $36.26(0.53)$ & $42.07(0.95)$ \\
\hline 14 & $22.75(6.64)$ & $16.05(7.69)$ & $16.33(3.86)$ & $65.91(3.61)$ \\
\hline
\end{tabular}




\begin{tabular}{ccccc}
15 & $13.75(4.10)$ & $28.19(7.10)$ & $14.52(2.16)$ & $69.36(2.23)$ \\
16 & $33.09(9.43)$ & $52.31(10.72)$ & $33.99(4.85)$ & $46.38(2.58)$ \\
17 & $15.99(5.68)$ & $33.09(6.15)$ & $23.64(4.07)$ & $58.59(5.89)$ \\
18 & $23.84(15.75)$ & $19.80(13.56)$ & $17.89(2.94)$ & $39.31(5.10)$ \\
19 & $25.56(5.91)$ & $30.69(4.38)$ & $36.05(7.58)$ & $30.89(9.38)$ \\
20 & $38.78(12.87)$ & $34.80(6.00)$ & $17.10(1.78)$ & $62.87(0.81)$ \\
21 & $39.56(9.81)$ & $31.51(7.57)$ & $38.26(5.40)$ & $47.16(2.65)$ \\
22 & $21.36(5.28)$ & $23.39(3.59)$ & $37.97(12.69)$ & $32.10(4.63)$ \\
23 & $24.23(7.48)$ & $23.25(5.56)$ & $30.79(10.93)$ & $30.21(4.15)$ \\
24 & $24.49(3.53)$ & $29.02(6.53)$ & $21.29(6.53)$ & $53.93(3.77)$ \\
25 & $24.40(4.40)$ & $33.71(7.16)$ & $40.80(10.79)$ & $50.51(7.73)$ \\
26 & $18.46(6.20)$ & $32.58(4.94)$ & $36.47(5.14)$ & $37.99(4.43)$ \\
27 & $18.27(6.71)$ & $16.00(7.09)$ & $31.94(5.46)$ & $47.60(4.46)$ \\
28 & $29.20(7.52)$ & $33.84(9.77)$ & $31.88(10.48)$ & $44.91(7.25)$ \\
29 & $26.84(4.37)$ & $20.19(5.88)$ & $33.91(5.65)$ & $37.25(3.52)$ \\
\hline Genome & $25.68(10.83)$ & $27.81(14.59)$ & $30.84(14.68)$ & $46.83(13.78)$ \\
\hline Wide & & &
\end{tabular}

\footnotetext{
* Computed using RFmix most likely assignment local ancestry estimates averaged across each window along the chromosome and throughout the genome. S.D. = standard deviation.
} 


\section{Table 3.5. Chromosome 5 Brahman proportion diverged regions and previously published associations. ${ }^{\mathrm{a}}$}

\begin{tabular}{|c|c|c|c|c|}
\hline Chr & $\begin{array}{l}\text { Coordinates } \\
\text { (Mb) }\end{array}$ & Breed & Identified Associations & Identified QTL \\
\hline 5 & $47.21-47.49$ & Brangus & $\begin{array}{l}\text { Milk/Udder: Milk fat yield [134], Milk protein } \\
\text { yield [134] }\end{array}$ & $\begin{array}{l}\text { Bilateral convergent strabismus with } \\
\text { exophthalmos [135], Milk yield [136] }\end{array}$ \\
\hline 5 & $47.49-48.92$ & $\begin{array}{c}\text { Brangus, Santa } \\
\text { Gertrudis, } \\
\text { Beefmaster }\end{array}$ & $\begin{array}{l}\text { Bovine respiratory disease susceptibility } \\
\text { [137] }\end{array}$ & \\
\hline 5 & $48.92-49.52$ & $\begin{array}{c}\text { Santa } \\
\text { Gertrudis, } \\
\text { Beefmaster }\end{array}$ & $\begin{array}{l}\text { Reproduction: Interval to first estrus after } \\
\text { calving [133], Pregnancy rate [138], Age at } \\
\text { puberty [133], Inhibin level [139] } \\
\text { Milk/Udder: Milk fat yield [134] } \\
\text { Meat/Carcass: Lean meat yield [140], }\end{array}$ & Udder swelling score [132] \\
\hline
\end{tabular}

\footnotetext{
a Significant windows were intersected with cattle dbQTL to identify regions overlapping published associations and QTL. Most significant regions for each American breed are in Table S3.1.
} 


\section{Table 3.6. Brahman proportion diverged regions and previously published associations for Beefmaster a.}

\begin{tabular}{|c|c|c|c|}
\hline Chr & $\begin{array}{l}\text { Coordinates } \\
\text { (Mb) }\end{array}$ & Identified Associations & Identified QTL \\
\hline 6 & $66.95-68.31$ & $\begin{array}{l}\text { Meat/Carcass: Fat thickness at 12th rib [124], Marbling score [126] } \\
\text { Milk/Udder: Milk fat yield [134] }\end{array}$ & $\begin{array}{l}\text { Average daily gain [127], Foot angle } \\
\text { [141], Milk protein percentage [142], } \\
\text { Milk yield [143] }\end{array}$ \\
\hline 8 & $\begin{array}{l}42.96-43.85 \\
45.40-45.96\end{array}$ & $\begin{array}{l}\text { Milk/Udder: Milk fat percentage [144], Milk fat yield [144], Milk } \\
\text { protein percentage [144], Milk protein yield [144], Milk yield [144] } \\
\text { Meat/Carcass: Longissimus muscle area [125], Muscle anserine } \\
\text { content [123] } \\
\text { Conformation: Rump angle [144], Strength [144] } \\
\text { Reproduction: Calving ease/Calving ease (maternal) [144] } \\
\text { Other: Net merit [144] }\end{array}$ & Residual feed intake [128] \\
\hline 25 & $0.04-4.86$ & $\begin{array}{l}\text { Reproduction: Age at first calving [145], Calving ease [146], } \\
\text { Conception rate [147], Daughter pregnancy rate [147], Early } \\
\text { embryonic survival [148], Fertility index [149], Fertilization rate [148], } \\
\text { Gestation length [146], Interval from first to last insemination [146], } \\
\text { Interval to first estrus after calving [146, 150], Reproductive } \\
\text { efficiency [145], Sire conception rate [151], } \\
\text { Growth: Birth weight [152], } \\
\text { Meat/Carcass: Carcass weight [123], Lean meat yield [140], } \\
\text { Subcutaneous fat [140], Yield grade [124], Palmitic acid content } \\
\text { [153] } \\
\text { Conformation: Body depth [150], Stature [150], } \\
\text { Milk/Udder: Milk caproic acid content [154], Milk fat yield [134, 150, } \\
\text { 155], Milk protein yield [150, 155], Milk yield [150, 155] } \\
\text { Other: Bovine respiratory disease susceptibility [137], Length of } \\
\text { productive life [147], Methane production [156], Net merit [147] }\end{array}$ & $\begin{array}{l}\text { Milk protein yield [149], Warner-Bratzler } \\
\text { shear force [157] }\end{array}$ \\
\hline
\end{tabular}

\footnotetext{
a Significant windows were intersected with cattle dbQTL to identify regions overlapping published associations and QTL. Most significant regions for Beefmaster are in Table S3.1.
} 
Table 3.7. Brahman proportion diverged regions and previously published associations for Brangus a.

\begin{tabular}{|c|c|c|c|}
\hline Chr & $\begin{array}{l}\text { Coordinates } \\
\text { (Mb) }\end{array}$ & Identified Associations & Identified QTL \\
\hline 20 & $\begin{array}{l}18.78-19.74 \\
20.02-20.38 \\
20.72-21.01\end{array}$ & $\begin{array}{l}\text { Reproduction: Conception rate [158], Daughter pregnancy rate } \\
\text { [144] } \\
\text { Growth: Yearling weight [125] } \\
\text { Milk/Udder: Milk fat percentage [159], Milk fat yield [159], Milk } \\
\text { profitability index [159], Milk yield [134], Udder } \\
\text { attachment/depth/height/weight [144] } \\
\text { Conformation: Body depth [144], Feet and leg conformation and } \\
\text { foot angle [144], Stature [144, 160], Strength [144], Rump width } \\
\text { [144] } \\
\text { Meat/Carcass: Carcass weight [124], , Intramuscular fat [161], Lean } \\
\text { meat yield [140] } \\
\text { Other: Abomasum displacement [162] }\end{array}$ & $\begin{array}{l}\text { Birth weight [130], Weaning weight } \\
\text { [129], Yearling weight [129], Carcass } \\
\text { weight [129], Heat tolerance [131], Milk } \\
\text { fat percentage [163], Milk protein yield } \\
\text { [164], Warner-Bratzler shear force [157] }\end{array}$ \\
\hline & $30.20-30.66$ & & \\
\hline 21 & $31.21-32.40$ & Milk/Udder: Milk Yield [134], Somatic cell score [165] & Average daily gain [166] \\
\hline
\end{tabular}

\footnotetext{
a Significant windows were intersected with cattle dbQTL to identify regions overlapping published associations and QTL. Most significant
} regions in Brangus are in Table S3.1. 
Table 3.8. Brahman proportion diverged regions and previously published associations for Santa Gertrudis a.

\begin{tabular}{ccll}
\hline Chr & $\begin{array}{c}\text { Coordinates } \\
(\mathbf{M b})\end{array}$ & \multicolumn{1}{c}{ Identified Associations } & \multicolumn{1}{c}{ Identified QTL } \\
\hline 16 & $\begin{array}{c}56.30-56.56 \\
57.09-58.36\end{array}$ & $\begin{array}{l}\text { Reproduction: Interval to first estrus after calving [133] } \\
\text { Carcass/Meat: Marbling score [167] } \\
\text { Milk/Udder: Milk fat percentage [168], Milk fat yield [134, 159] }\end{array}$ & $\begin{array}{l}\text { Calving ease (maternal) [132], Carcass } \\
\text { weight [130] }\end{array}$ \\
& & &
\end{tabular}
a Significant windows were intersected with cattle dbQTL to identify regions overlapping published associations and QTL. Most significant
regions in Brangus are in Table S3.1. 


\title{
CHAPTER 4
}

\section{GENOME-WIDE ASSOCIATION ANALYSIS FOR BREED DIFFERENCES IN FEED EFFICIENCY, GROWTH AND COMPONENT TRAITS}

\begin{abstract}
Background

Although feed efficiency was recognized as a vitally important economic trait in beef production more than 40 years ago, understanding the genetic architecture and detecting associations between genetic markers and feed efficiency remains an area of active research with relevance to current production systems. Several genome-wide association analyses have now been conducted in beef cattle for feed efficiency and component traits, however, these analyses have primarily been conducted within specific breeds detecting little overlap of identified QTL across populations. We conducted the largest genome-wide association analyses performed to date for feed efficiency and component traits in beef cattle. We evaluate the significance of alternative models when additive and heterotic breed effects were included in the model.
\end{abstract}

\section{Results}

Using CRUMBLER breed composition estimates for 11,505 animals with feed efficiency and component trait phenotypes, we evaluated the significance of genome-wide association analysis models for each phenotype, when breed effects were not included (Model 1), additive breed effects were included (Model 
2), or additive breed effects and interactions between breeds representing heterotic effects were included (Model 3 ) in the analytical model. Additive and heterotic effects were significant for all traits $(p<0.05)$, however the genomewide association analyses indicated no substantial differences in detected QTL across models for any phenotype. We did not detect novel QTL, however, we found pleiotropic associations for previously identified QTL that had not previously been detected. We also show that previously identified QTL, found not to overlap in separate breed analyses, coalesce in an across breed analysis when breed effects are included. However, further investigation is necessary to explain the causes of the inflated $p$-values in the $Q-Q$ plots when breed differences and pedigree relatedness are explained in the analytical model.

\section{Conclusions}

Feed is the most costly component of the production of beef. Consequently, beef producers continue to select on traits that are related to the efficient use of feed and researchers continue the quest to identify the genetic variants underlying feed efficiency and its component traits. Recording feed efficiency phenotypes is expensive but as more data become available and access to large scale genome-wide association data become available, causal variants influencing efficiency will be identified and molecular breeding values with utility across breeds will be generated. 


\section{Background}

In U.S. beef production systems, particularly feedlots, the cost of feed comprises more than $75 \%$ of the total input costs, making feed expenditures the most costly component of the production of beef [169-171]. Although feed efficiency was recognized as a vitally important economic trait in the production of beef more than 40 years ago, it remains an active area of research that remains extremely relevant to current beef production $[169,170]$. Producers and researchers both continue the quest to develop methods to identify and enable the selection of more efficient animals to benefit the entire production system, including consumers $[171,172]$. Selection for more efficient cattle has become an important social issue since efficient cattle emit fewer greenhouse gases and require less land and water resources for each pound of produced beef [50]. It has been estimated that a $10 \%$ increase in the efficiency of utilization of feed by U.S. beef cattle would result in an annual cost savings of more than U.S. $\$ 1$ billion, motivating efforts to gain further insights into the efficiency of feed utilization in beef cattle [51].

Feed intake is difficult to measure and historically cattle have been selected for the gross efficiency of growth via selecting for increased yearling weights. This has the undesirable consequence of producing larger mature body weights in females, which results in increased maintenance and nutrient costs for producers, as well as potentially increased birth weights of calves which may result in calving difficulties and dystocia [173]. When feed intakes can be 
measured, feed conversion ratio, the ratio of feed consumed to body weight gain, has traditionally been used to measure feed efficiency and enable the selection of more efficient cattle [172]. However, selection on a ratio of traits can produce unexpected results and residual feed intake (RFI), has been proposed as an alternative method of measurement of feed efficiency since it is a linear index of intake, growth and maintenance feed requirement traits. Use of RFI as a measure of feed efficiency has gained popularity because it is phenotypically independent of the traits used to produce the measurement. RFI is the difference between expected and actual feed intake based on an animal's maintenance requirement (related to body weight) and growth rate during the feeding period $[170,172]$. RFI has become the preferred measurement of feed efficiency, due to the substantial phenotypic variation and the moderate to high estimated heritabilities that have been reported for RFI in cattle populations, ranging from 0.08 to $0.49[50,174-178]$.

The economic importance of feed efficiency and the desire to create reliable genomic selection techniques for increased efficiency of feed utilization and growth rates in cattle have motivated several linkage studies and genome wide association studies (GWAS) [50, 51, 128, 179-182]. Relatively large GWAS have been conducted with high-density genotype data, including data produced using the Illumina BovineSNP50 (50K) and Illumina BovineHD (778K) assays. These GWAS have evaluated QTL across several cattle breeds but have generally been conducted within specific breeds, such as Angus, Hereford and 
Simmental x Angus $[50,51]$. However, these breed assignments have been based upon historical breeding information concerning the breeds used to form specific herds or the assumption that black-hided feeder cattle sourced from regional sale yards will be primarily Angus in origin [68]. Individual animal breed composition data are generally not available for animals from commercial beef production where crossbreeding is routinely practiced. However, such knowledge would allow the opportunity to determine the magnitude of breed additive and heterotic effects on feed efficiency and it's component traits. Commercially sourced animals are frequently used to generate resource populations to study and collect phenotypic information on economically relevant traits such as bovine respiratory disease [52] and feed efficiency $[50,51]$. The complex ancestral history and extensive admixture present in these crossbred resource populations may impact downstream GWAS and lead to the development of genomic prediction models for populations in which the breed composition is not completely understood $[50,51]$.

GWAS performed in different samples of cattle from putatively different breed groups have identified significant QTL for average daily gain, dry matter intake, metabolic mid-test body weight, and residual feed intake. However, little overlap between breeds has been detected for these QTL and it is unclear whether this is because differences in patterns of linkage disequilibrium influence their detection, or if the genetic architecture of traits differs among breeds [50,51]. These analyses have assumed that the analyzed samples are somewhat genetically 
homogeneous, but the extent of differences in admixture between individuals within each analyzed group has not been known. Crum et al. (2019) have recently developed a tool to estimate the breed composition of admixed cattle based on a reference panel comprised of 17 curated breeds of Bos taurus taurus and Bos taurus indicus cattle prevalent in the United States [68]. The objective of this study was to incorporate individual animal breed composition information along with genomic relationship information into the GWAS of RFI and its growth, maintenance and intake components: average daily gain (ADG; lb/d), average daily dry matter intake (DMI; lb/d), and mid-test metabolic body weight (MMWT; $\left.\mid \mathrm{b}^{0.75}\right)$ in the largest sample of animals evaluated for feed efficiency to date $(n=11,505)$. We examine the effects of including breed additive and heterotic effects on chip-heritability and variance component estimates for these traits when breed composition was estimated using CRUMBLER [68]. Additionally, we examined the nature of QTL that were detected in this analysis with those previously identified in analyses of subsets of the data.

\section{Materials and Methods}

Cattle Populations, Phenotypes and Genotypes

Growth and feed efficiency data were collected with the assistance of commercial beef producers and with the approval of the Animal Care and Use Committees of the University of Missouri (ACUC Protocol 7505), the University of Illinois Champaign-Urbana (IACUC Protocols 06091 and 09078), and the US Meat Animal Research Center. The animals were collected as part of the USDA NIFA 
"National program for genetic improvement of feed efficiency in beef cattle" project (FE project) and descriptions of the genotyping and methods related to the collection of phenotypes have previously been described [50]. The cattle included in the present study were genotyped on at least one of 7 different genotyping platforms currently used internationally to genotype cattle, including the GeneSeek (Lincoln, NE) GGP-90KT, GGP-F250, and GGP-LDV3 assays, the Illumina (San Diego, CA) BovineHD and BovineSNP50 assays, the Irish Cattle Breeding Federation (Cork, Ireland) IDBv3, and GeneSeek (Lincoln, NE) BOVG50v1 assays (Table 4.1). In total, 13,477 samples were genotyped and concordance was evaluated for individuals genotyped more than once, the variants common to the assays were merged and discordant genotypes were removed. Samples genotyped using more than one assay primarily were regenotyped when the GGP-F250 assay was developed since this assay was designed to be enriched for variants within previously detected feed efficiency QTL regions $[50,51]$.

PLINK1.9 [77] was used to filter variants and individuals. The SNP positions were based on the ARS-UCD1.2 bovine reference genome assembly [98]. Nonautosomal variants were removed from the data. Variants and individuals with call rates $<0.90$ were also removed. Genotypes were phased by Eagle 2.4 [99] using a reference panel of haplotypes from 9,937 individuals genotyped with the Illumina (San Diego, CA) BovineHD (HD) assay. Phased haplotypes were then imputed with Minimac3 [100] to the union of two high-density research assays; 
the HD and the GGP-F250 (GeneSeek, Lincoln, NE) (F250). A multi-breed imputation reference was created as in Rowan et al. (2019) [101]. The reference panel contained 2,719 animals genotyped with both the F250 and the HD assays, 25,772 animals genotyped only with the F250, and 7,218 animals genotyped only with the HD assay. Following imputation, each sample had genotypes for 836,118 variants and 11,505 individuals remained for analysis following imputation.

Breed composition of the 11,505 individuals was estimated using a multi-breed reference panel and the CRUMBLER global ancestry pipeline as described in Crum et al. (2019) [68]. Breed composition estimates were produced on a per individual basis and were normalized for each individual to remove breeds with assignment estimates of $<5 \%$. The means and standard deviations for the FE individuals and each of the CRUMBLER reference populations are presented in Table 4.2. Distributions of CRUMBLER breed assignment by breed are shown in Figure 4.1.

\section{Models}

Genome-wide association studies were performed using a univariate linear mixed model, implemented in GEMMA [183]. The linear mixed model used in GEMMA for this study can be generally specified as:

$$
y=W \boldsymbol{\alpha}+X \boldsymbol{\beta}+u+\boldsymbol{\varepsilon} ; u \sim M V N_{n}\left(0, \lambda \boldsymbol{\tau}^{-1} K\right), \boldsymbol{\varepsilon} \sim \operatorname{MVN}_{n}\left(0, \boldsymbol{\tau}^{-1} / n\right)
$$


where, $y$ is an $n$-vector of quantitative trait observations for $n$ individuals; $W$ is an $n x c$ matrix of covariates fit as fixed effects including an identity intercept column of $1 \mathrm{~s} ; \boldsymbol{\alpha}$ is a $c$-vector of corresponding coefficients including the intercept; $\mathrm{X}$ is an $n$-vector of marker genotypes; $\boldsymbol{\beta}$ is the marker effect size; $u$ is a vector of random effects; $\boldsymbol{\varepsilon}$ is an $n$-vector of errors; $\boldsymbol{\tau}^{-1}$ is the variance of residual errors; $\lambda$ is the ratio between the two variance components; $\mathrm{K}$ is an $n \times n$ genomic relationship matrix; $I_{n}$ is an $n \times n$ identity matrix and $M V N_{n}$ represents the multivariate normal distribution of dimension $n$. Wald, likelihood ratio, and score test statistics are used by GEMMA to test the alternative hypothesis that there are marker effects for the trait of interest $\left(H_{1}: \boldsymbol{\beta} \neq 0\right)$ against the null hypothesis of no marker effects $\left(H_{0}: \boldsymbol{\beta}=0\right)$. The restricted maximum likelihood estimate $(\mathrm{REML})$ of $\lambda$ and maximum likelihood estimates (MLE) of each $\boldsymbol{\beta}$ in the model are computed along with $p$ values for evaluating the significance of the fitted effects.

We compared 3 models for the feed efficiency traits RFI, ADG, DMI and MMWT, incorporating either no effect for breeds (Model 1), additive breed effects (Model 2), or heterotic interactions between pairs of breeds (Model 3), respectively. RFI was analyzed by including partial linear regressions on ADG and MMWT in the models used to evaluate DMI [50]. Due to the significant number of contemporary groups $(n=586)$ represented in the data, where contemporary group was defined by the combination of feeding location and origin of the cattle, season of the year started on feed, year the animal was fed, pen, treatment, ration fed, and sex of the animal, we adjusted the observations prior to GEMMA 
analysis using a REML mixed model approach as implemented in the MatrixModels package in $\mathrm{R}$. The residual phenotypes from this analysis were then used in the subsequent GEMMA analyses.

The null model, Model 1 can be specified as:

$$
\begin{gathered}
y=\boldsymbol{\alpha}+X \boldsymbol{\beta}+u+\boldsymbol{\varepsilon} \\
u \sim \operatorname{MVN}_{n}\left(0, \lambda \boldsymbol{\tau}^{-1} K\right), \boldsymbol{\varepsilon} \sim \operatorname{MVN}_{n}\left(0, \boldsymbol{\tau}^{-1} I_{n}\right) .
\end{gathered}
$$

where $X, \boldsymbol{\beta}$, and $u$ are defined as stated in the GEMMA generalized linear mixed model above.

Model 2, can be specified as:

$$
\begin{gathered}
y=\boldsymbol{\alpha}+\sum_{i=1}^{n} \text { Breed }_{i} \alpha_{i}+X \boldsymbol{\beta}+u+\boldsymbol{\varepsilon} \\
u \sim \operatorname{MVN}_{n}\left(0, \lambda \boldsymbol{\tau}^{-1} K\right), \boldsymbol{\varepsilon} \sim \operatorname{MVN}_{n}\left(0, \boldsymbol{\tau}^{-1} I_{n}\right),
\end{gathered}
$$

where $\mathrm{Breed}_{i}$ are additive breed effects fit as partial regression coefficients on CRUMBLER estimated breed proportions $\boldsymbol{\alpha}_{i}$ for the animals in the FE population.

Finally, Model 3, can be specified as:

$$
\begin{gathered}
y=\boldsymbol{\alpha}+\sum_{i=1}^{n} \text { Breed }_{i} \alpha_{i}+\sum_{i=1}^{n} \text { Breed }_{i j} \alpha_{i} \alpha_{j}+X \boldsymbol{\beta}+u+\boldsymbol{\varepsilon} ; \\
u \sim \operatorname{MVN}_{n}\left(0, \lambda \boldsymbol{\tau}^{-1} K\right), \boldsymbol{\varepsilon} \sim \operatorname{MVN}_{n}\left(0, \boldsymbol{\tau}^{-1} I_{n}\right),
\end{gathered}
$$

where Model 3 incorporates interaction terms for evaluating heterotic interactions between breeds using CRUMBLER estimated breed proportions for the FE population. 
The effect of including breed composition effects in the model were evaluated by comparing the goodness of fit of each of the models sequentially for all feed efficiency and component traits. Population structure due to relatedness between individuals was accounted for in each model using a centered relationship matrix as implemented in GEMMA [183]. Using the qqman R package, QuantileQuantile (Q-Q) plots were generated to detect unaccounted population structure or kinship and to assess the power of the analyses to detect QTL and finally, Manhattan plots were created to visualize significant SNP associations.

\section{Reporting Statistics}

GEMMA reports PVE, proportion of variance explained by the typed SNPs for the phenotype being analyzed, sometimes known as "chip heritability" [183]. Previous GWAS studies for feed efficiency and related traits in beef cattle report a pseudo heritability estimate, by $h^{2}{ }_{a}=\sigma^{2} a /\left(\sigma^{2}{ }_{a}+\sigma^{2}{ }_{e}\right)[184,185]$. However due to the use of a centered relationship matrix in this analysis on feed efficiency, the two estimates cannot be considered equal, as the PVE is based on the mean of the diagonal elements of the relationship matrix, which is not equal to 1 unless a standardized kinship matrix is computed [186]. Pseudo-heritability estimates were generated for each model and phenotype through GEMMA reported variance components, to compare our results with those previously reported. Models were compared for goodness of fit using a chi-squared likelihood ratio test with degrees of freedom equal to the difference in number of fitted effects 
between models. Models 1 and 2 which test for additive breed differences, were compared using a $x^{2}$ statistic with $n$ degrees of freedom, where $n$ is the number of breed effects included in Model 2. Similarly, the existence of heterotic effects was evaluated by comparing Models 2 and 3 , using a $x^{2}$ statistic with $n^{\star}(n-1) / 2$ degrees of freedom, where $n^{\star}(n-1) / 2$ is the number of heterotic effects included in Model 3.

\section{Results and Discussion}

Three sets of GEMMA analyses were conducted using phenotypes that had been precorrected for contemporary group effects. The null model (Model 1) included only an overall mean, whereas Model 2 included additive breed effects, and Model 3 additive breed effects and pairwise interaction terms among breed effects. CRUMBLER estimates of breed composition were used to define the breed composition variables analyzed in Models 2 and 3 . Boxplots showing the breed composition distributions for the 11,505 FE animals are in Figure 4.1. CRUMBLER estimates breed composition for 17 breeds and many of these breeds were not significantly represented in the genomes of the 11,505 FE animals. Consequently, we selected the breeds that had the highest dispersion in CRUMBLER estimates among the samples, to avoid issues of muticollinearity in the estimation of the breed effects. Consequently, the partial regressions for each fitted breed effect are estimates of the difference between that fitted effect and the sum of the effects for the 10 breeds that were not fitted in the model. The 7 breeds with the highest dispersion in these samples were Angus, Charolais, 
Hereford, Gelbvieh, Limousin, Red Angus and Simmental (Table 4.1). While we did not use any a-priori knowledge of breed assignment to select those breeds that should be fit in these models, these 7 breeds were among the most commonly identified in the phenotype file.

Significance of Additive Breed Effects (Model 2)

GEMMA outputs the value of the log-likelihood of the data at the maximum likelihood estimates (MLE) for each of the models and trait analyses. Loglikelihood values for Models 1 and 2 and MLE of the additive breed effects for Model 2 are reported in Table S4.1 and Table S4.2, respectively. Models 1 and 2 were compared using a $x^{2}$ statistic with 7 degrees of freedom, as there were 7 breed effects incorporated into Model 2 that were not present in Model 1, and indicated that the additive breed effects were significant $(p \leq 0.05)$ for all traits (Table 4.3).

Manhattan plots of genome-wide GWAS results revealed few differences in QTL between the two models for all of the traits (Figure 4.2, 4.3, 4.4, 4.5). The QTL for ADG at BTA10_16 Mb is genome-wide significant in Model 1, but has reduced significance in Model 2 (Figure 4.2). Likewise, a significant QTL for DMI at BTA14_27 Mb found in Model 1, falls below the genome-wide significance threshold when breed effects were included in the model in Model 2 (Figure 4.3). Additionally, Figure 4.6 shows the Q-Q plot for DMI p-value estimates, which appear to be inflated, suggesting that some additional population structure 
remains unexplained in Models 1 and 2. Further investigation into the causes of this deviation from expectation will be necessary to assess the reliability of the DMI GWAS results.

Significance of Heterotic Effects (Model 3)

Models 2 and 3 were evaluated for the significance of heterotic effects (interactions between pairs of breeds) using a $x^{2}$ distribution with 21 degrees of freedom, the number of interaction terms included in Model 3 and found to be significant $(p \leq 0.05)$ (Table S4.2 and Table S4.3). This is an interesting finding, as the majority of fed cattle originate from commercial herds that have capitalized on the use of crossbreeding systems to capture gains due to heterosis, yet to our knowledge, this is the first time that heterostic effects have been incorporated into a cattle GWAS. Again, GWAS QTL results for all traits differed little between Models 2 and 3 (Figures 4.2, 4.3, 4.4, 4.5).

\section{GWAS Results}

Psuedo-heritabilities and variance component estimates are reported in Table 4.4 for RFI, ADG, DMI, and MMWT for each of the evaluated models. Psuedoheritability is the proportion of phenotypic variation that is explained by the set of SNP markers incorporated into a high-density genotyping assay [51, 187, 188]. Here it reflects the SNPs present on the union of the $778 \mathrm{~K}$ assay, which primarily includes common variants, and the F250 assay which includes a large number of rare variants primarily located in coding regions of the genome. Little variation 
exists between the estimates across models. Estimates of pseudo-heritability were 0.45 for RFI, $0.41-0.42$ for $\mathrm{ADG}, 0.50$ for $\mathrm{DMI}$, and 0.56 for MMWT. These estimates are similar to those found in previous analyses of subsets of these data $[50,51]$. The estimates presented here are among the highest reported for $A D G$ and $D M I$, possibly due to the increased genetic diversity that comes from a substantially larger sample size of admixed animals. The moderate to high pseudo-heritability estimates obtained for all traits across all models in these analyses indicate the potential for the identification of novel QTL, particularly considering the sample size used in this study. For all models, Q-Q plots (Figure 4.6-4.9) suggest either a well powered study, due to the large sample set and high density of imputed genotypes, or the potential for unaccounted population structure within the data (genomic inflation factors are reported in Table S4.4).

Except for DMI, where no strongly significant QTL were detected in any of the fitted models, we report a QTL on BTA20 at 4.6-5.0 Mb that is strongly associated with ADG, MMWT and RFI. A QTL in this region was previously identified in a Hereford sample [50] as being associated with RFI and MMWT and is reported here for the first time as also being associated with ADG. Two potential positional candidate genes have been identified as being biologically important within this QTL region, including genes STC2 [50] and LOC10190982 (SYNPO2 like) [51]. While the physiological role of STC2 in cattle is unknown, it is known to be expressed in developing avian striated muscle and joints [189] and it's overexpression has been found to reduce intramembranous and 
endochondral bone development and skeletal muscle growth in mice and cause a dwarf phenotype in transgenic mice [190]. STC2 is an endoplasmic reticulum stress response gene which has been associated with adiposity and obesity in nondiabetic humans [191]. Other endoplasmic reticulum-related proteins also reside within this QTL region including ERCIC1, CREBRF and BNIP1 [51]. Due to its association with myopathy related proteins, SYNPO2 is considered a candidate gene for muscle disease and has a role in the early development of skeletal muscle [192].

Two significant pleiotropic QTL located at BTA14_23 Mb and BTA6_37-38 Mb affect ADG and MMWT and were detected in all fitted models. A QTL at 14_24$25 \mathrm{Mb}$ has previously been identified within a Simmental $x$ Angus subset of these data $[50,51]$ and is supported by associated SNPs located $\geq 1 \mathrm{Mb}$ apart [51]. However, this is the first report of the association with this region of BTA14 and ADG. This region on BTA14 warrants further investigation due to its known associations with birth weight in Nellore cattle, variation in human height (orthologous human locus), variation in body stature in beef and dairy cattle [193-195], carcass weight in Japanese Black cattle [196], and subcutaneous rump fat thickness in cattle $[180,197]$. QTL identified near BTA6_37-38 Mb influencing ADG and MMWT have previously been identified [50, 51]. This genomic region has been identified as significant in cattle studies many times, as it is known to harbor QTL influencing growth, body weights, calving ease, reproductive, milk, carcass and feed efficiency traits [50]. 
The QTL at BTA7_90.8 Mb was identified as being associated with both ADG and RFI and has not previously been reported. A QTL at 7_93 Mb has been identified as associated with ADG and MMWT in previous analyses $[50,51]$ and was reported as the second-largest effect QTL associated with body weights at all stages of development in beef cattle and is segregating in a large number of breeds [167]. Located in this region is $A R R D C 3$ (arrestin domain containing 3), a member of the arrestin super family that regulates obesity in mice and human males and energy expenditure [198]. Beta-adrenergic agonists are often supplemented in the development of cattle, pigs, poultry, and sheep to increase muscle deposition and decrease fat accumulation [199, 200] and the arrestin family of proteins are signaling proteins controlling metabolism through the desensitization of beta-adrenergic receptors. The previous analyses that identified BTA7_93 Mb as being associated with ADG and MMWT also identified that this region is telomeric of $A R R D C 3$ a candidate for this published QTL association $[50,51]$. These previous analyses were conducted using the UMD3.1 bovine reference assembly [201], whereas the analyses we conduct here are based upon the new ARS_UCD1.2 bovine reference assembly [98]. The new assembly identifies ARRDC3 near BTA7_90 Mb, suggesting that our identified QTL and the previously published QTL BTA7_93 Mb QTL are identical. 


\section{Conclusions}

Feed efficiency and its component traits have long been targets for selection by beef cattle producers. In U.S. beef production systems, the cost of feed comprises more than $75 \%$ of the total input costs of production, making the feeding of cattle the most costly component of beef production $[170,171]$. Previous GWAS have been performed with high density genotype data, for feed efficiency and component traits. These GWAS have considered several breeds of cattle but have primarily used a-priori information to partition the data in order to conduct analyses within subsets that are relatively genetically homogeneous with respect to breed. However, crossbreeding is extensively used in commercial beef production a-priori information concerning breeding programs or coat color of the animals cannot effectively cluster animals into groups that might be considered to be panmictic. Using CRUMBLER breed composition estimates for 11,505 individuals, we first fit GWAS models for feed efficiency and component traits that did not include breed effects (Model 1). Additive breed effects were next included in the model as covariates (Model 2), and finally, additive breed effects and pairwise interactions representing heterotic effects were included in the model as covariates (Model 3). Likelihood ratio tests were used to compare the goodness of fit of the models and the breed additive and heterotic effects were found to be significant for all analyzed traits $(p<0.05)$, however, the GWAS results revealed only minor effects on the identification of QTL across the models for each trait. 
To our knowledge, this is the largest GWAS performed for feed efficiency and component traits performed in cattle to date. Unlike many of the previously published GWAS in beef cattle, we report results using high density imputed data with SNPs mapped to the new ARS_UCD1.2 bovine reference assembly. Previously identified QTL have had little overlap across populations. While we did not detect evidence for novel QTL, we extended the range of pleiotropic effects of previously identified QTL, and provide evidence that previously identified QTL can be detected in an across breed analysis when breed effects are explained in the analytical model. Previously reported estimates for genomic inflation factors for an analysis with $113 \mathrm{~K}$ individuals and a trait heritability of 0.5 are aligned with the inflation factors we report here [202]. However, further investigation may be necessary to rule out any effect of unexplained population structure in the data, which may contribute to the lack of detection of large effect QTL for DMI. 


\section{Figures}

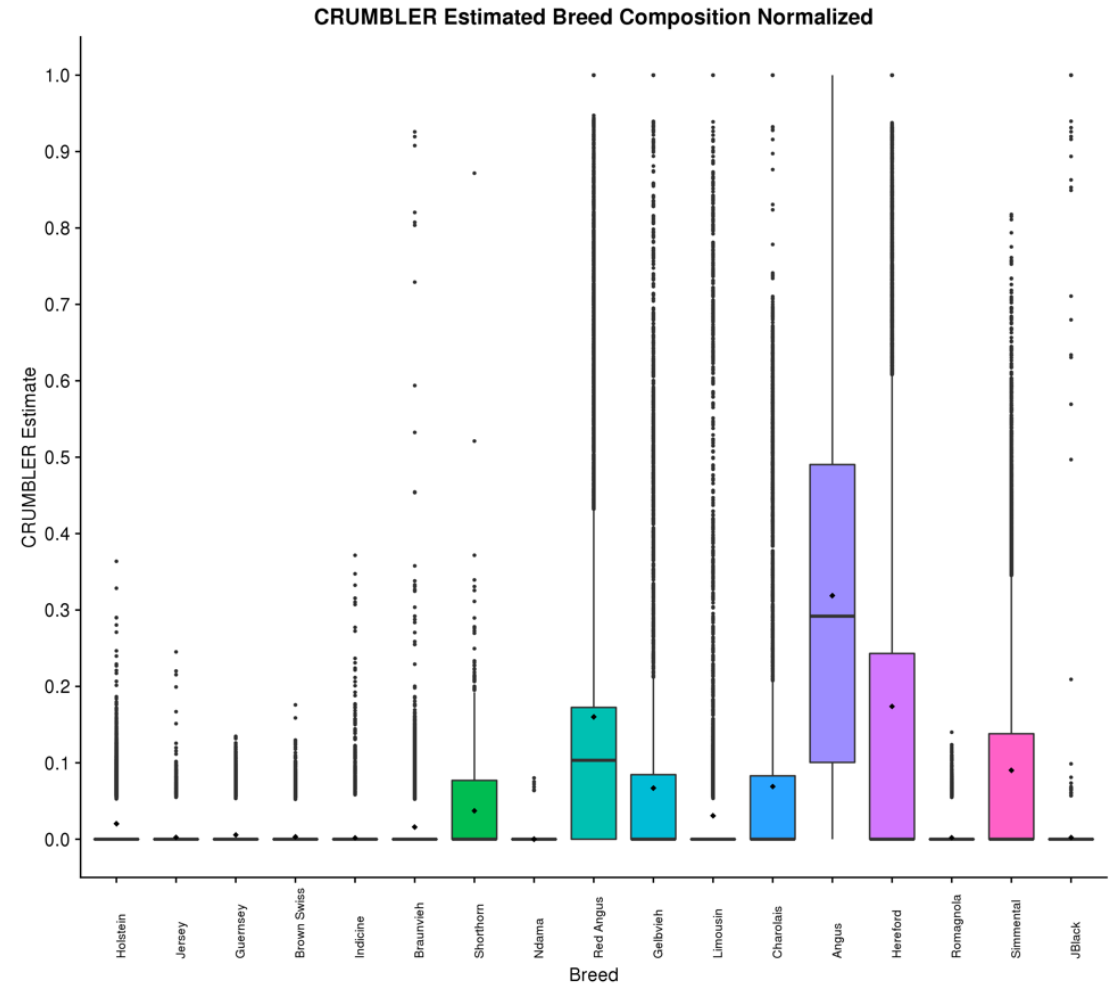

Figure 4.1. Distributions of Normalized CRUMBLER Breed Composition Estimates for FE Individuals. Boxplots of distributions for CRUMBLER estimated breed compositions following normalization for individual estimates $<5 \%$ for a breed. 
a.

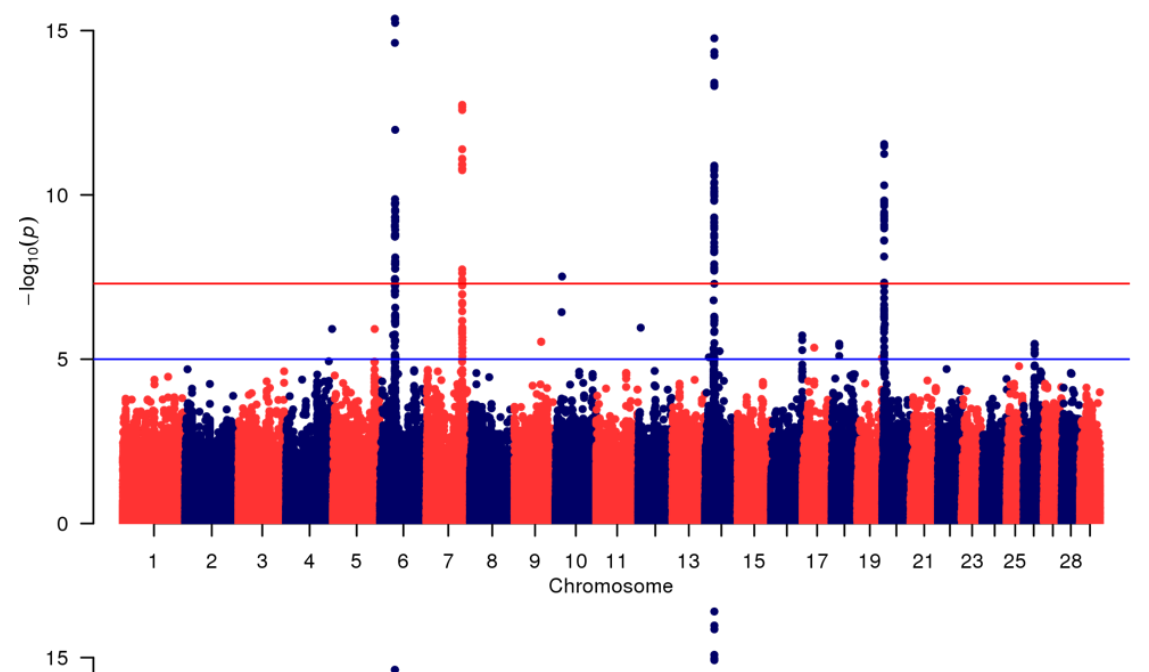

b.

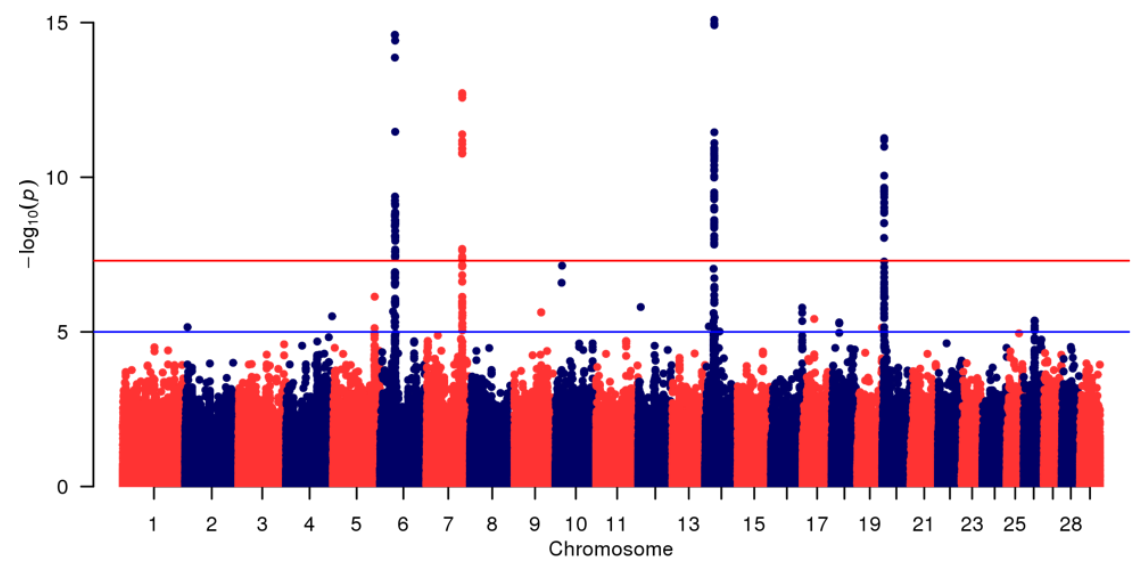

C.

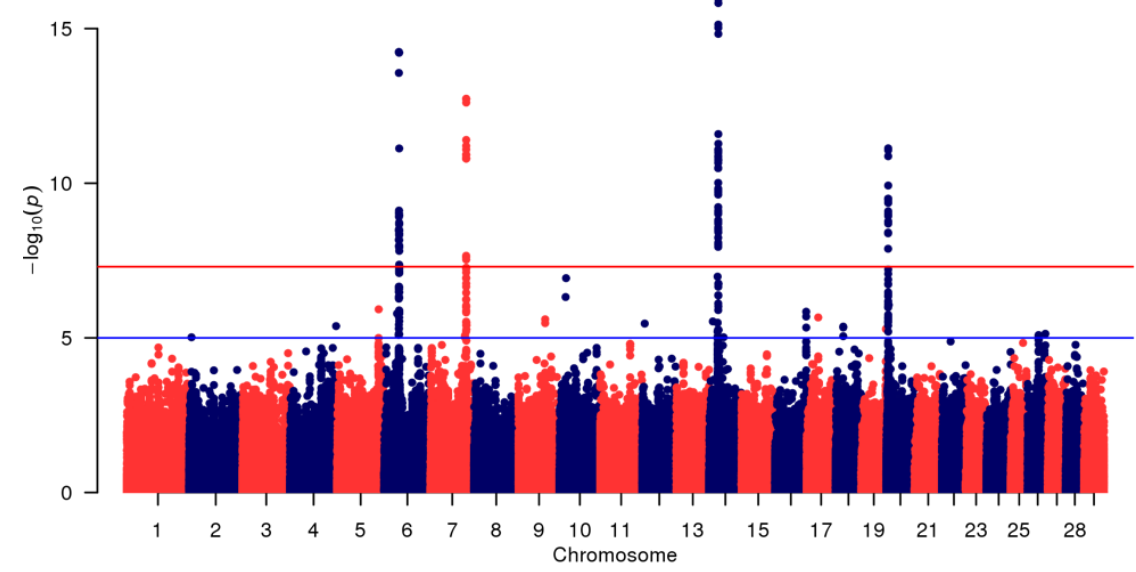

Figure 4.2. Manhattan Plots for Average Daily Gain GWAS. (a.) Model 1 which analyzed contemporary group adjusted data. (b). Model 2, which included additive breed effects. (c). Model 3, which included additive breed effects and pairwise breed interactions to evaluate heterosis. 
a.

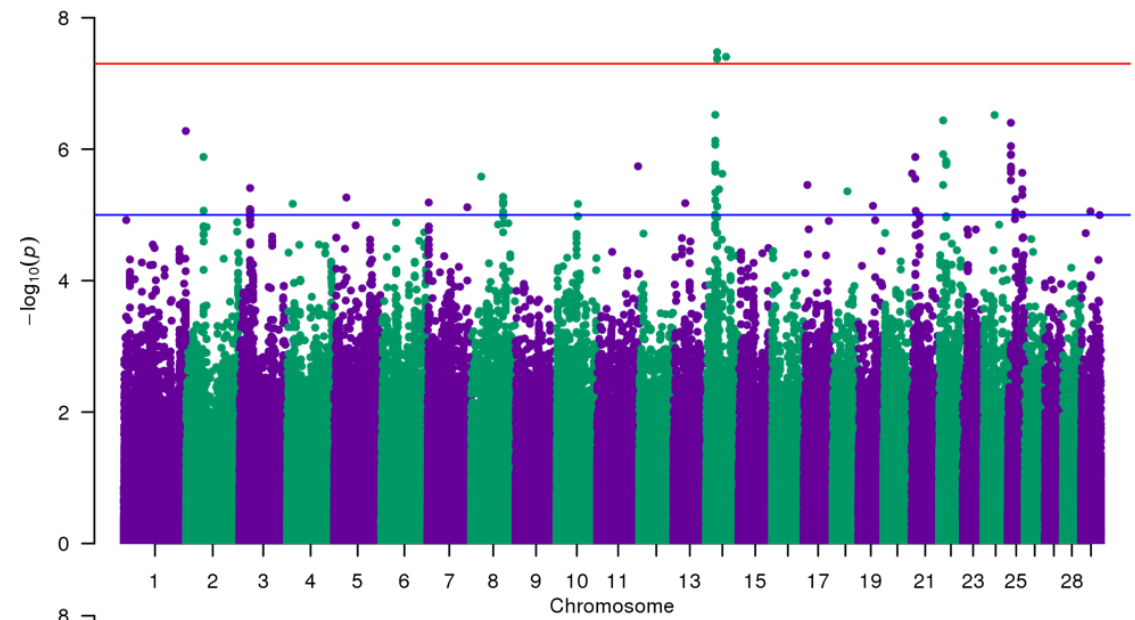

b.

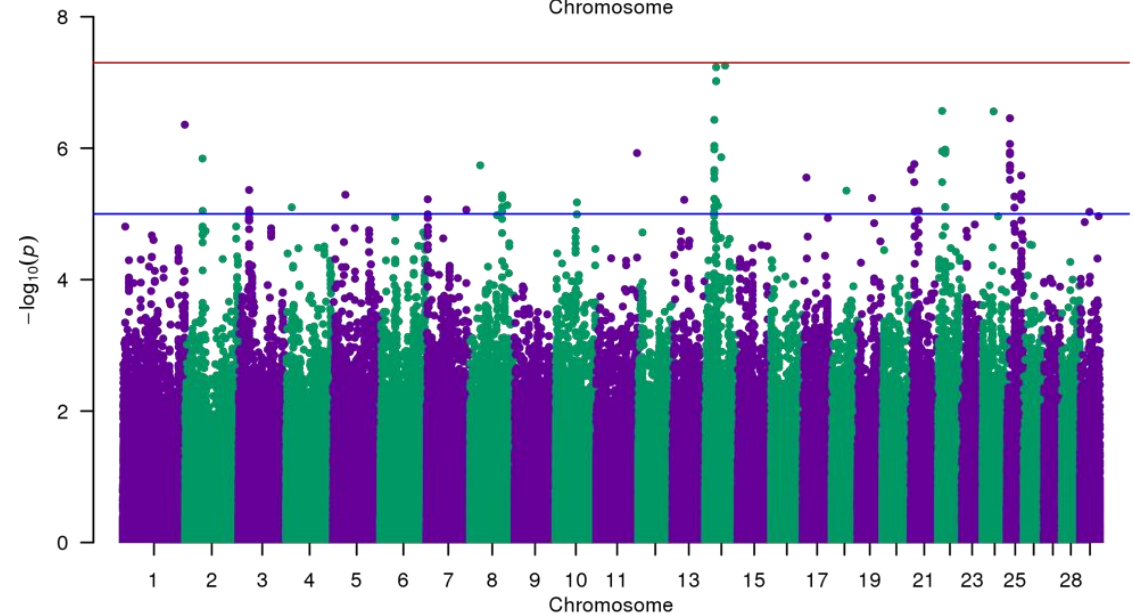

C.

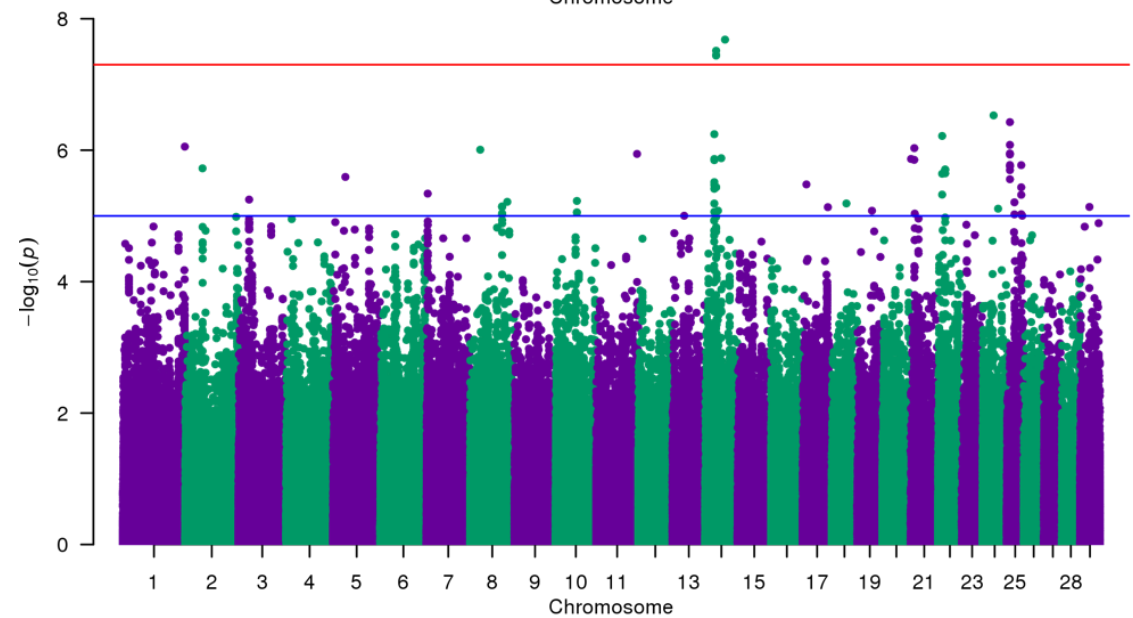

Figure 4.3. Manhattan Plots for Dry Matter Intake GWAS. (a.) Model 1 which analyzed contemporary group adjusted data. (b). Model 2, which included additive breed effects. (c). Model 3, which included additive breed effects and pairwise breed interactions to evaluate heterosis. 
a.

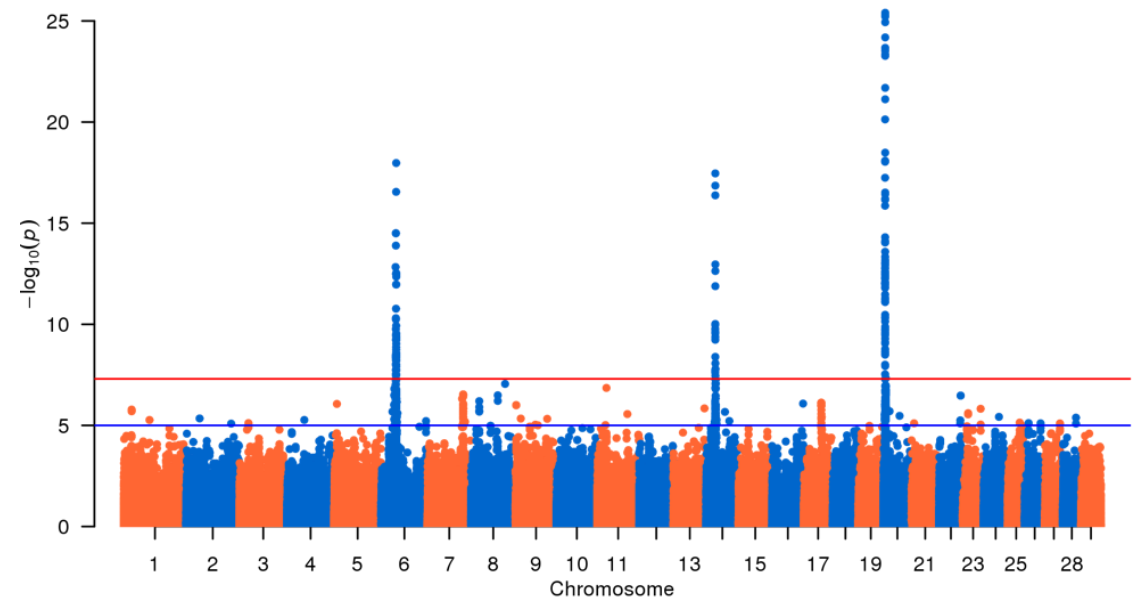

b.

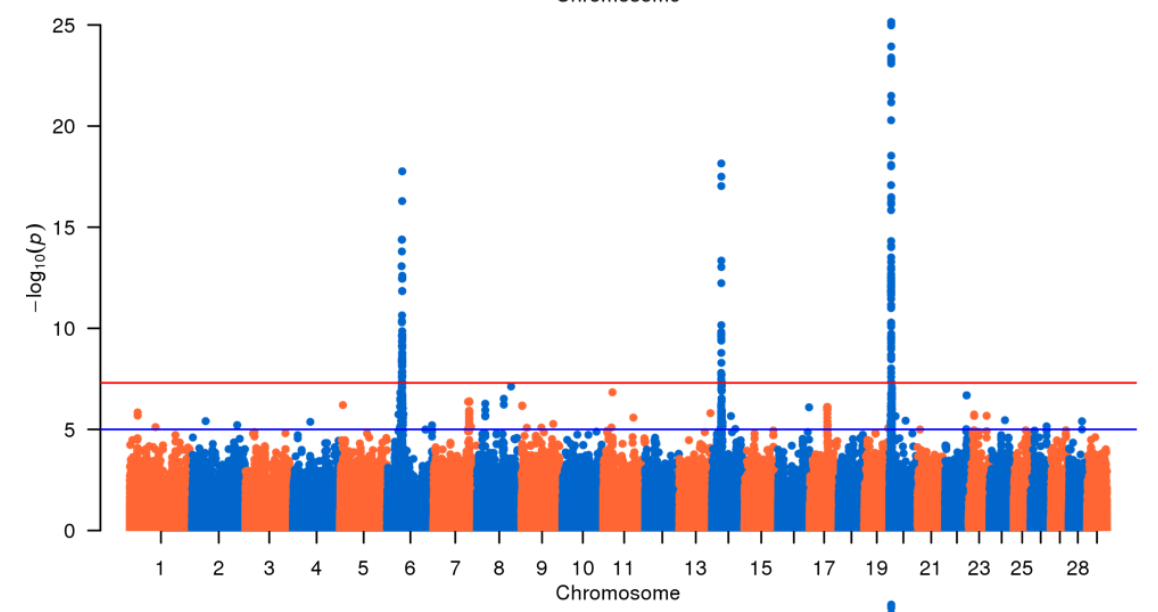

C.

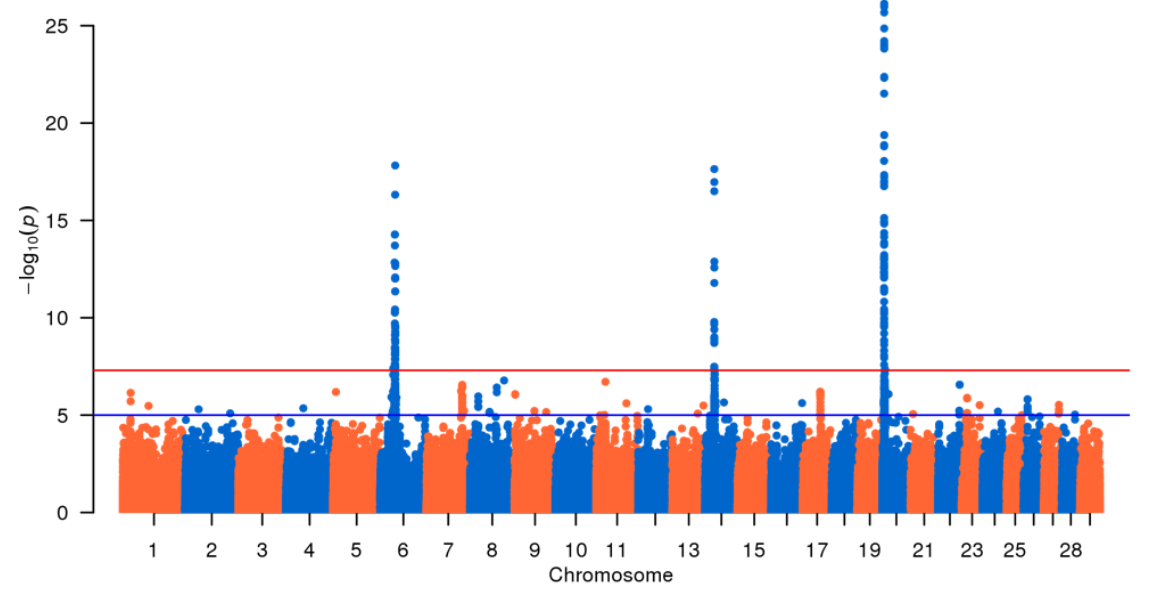

Figure 4.4. Manhattan Plots for Metabolic Mid-Weight GWAS. (a.) Model 1 which analyzed contemporary group adjusted data. (b). Model 2, which included additive breed effects. (c). Model 3, which included additive breed effects and pairwise breed interactions to evaluate heterosis. 
a.
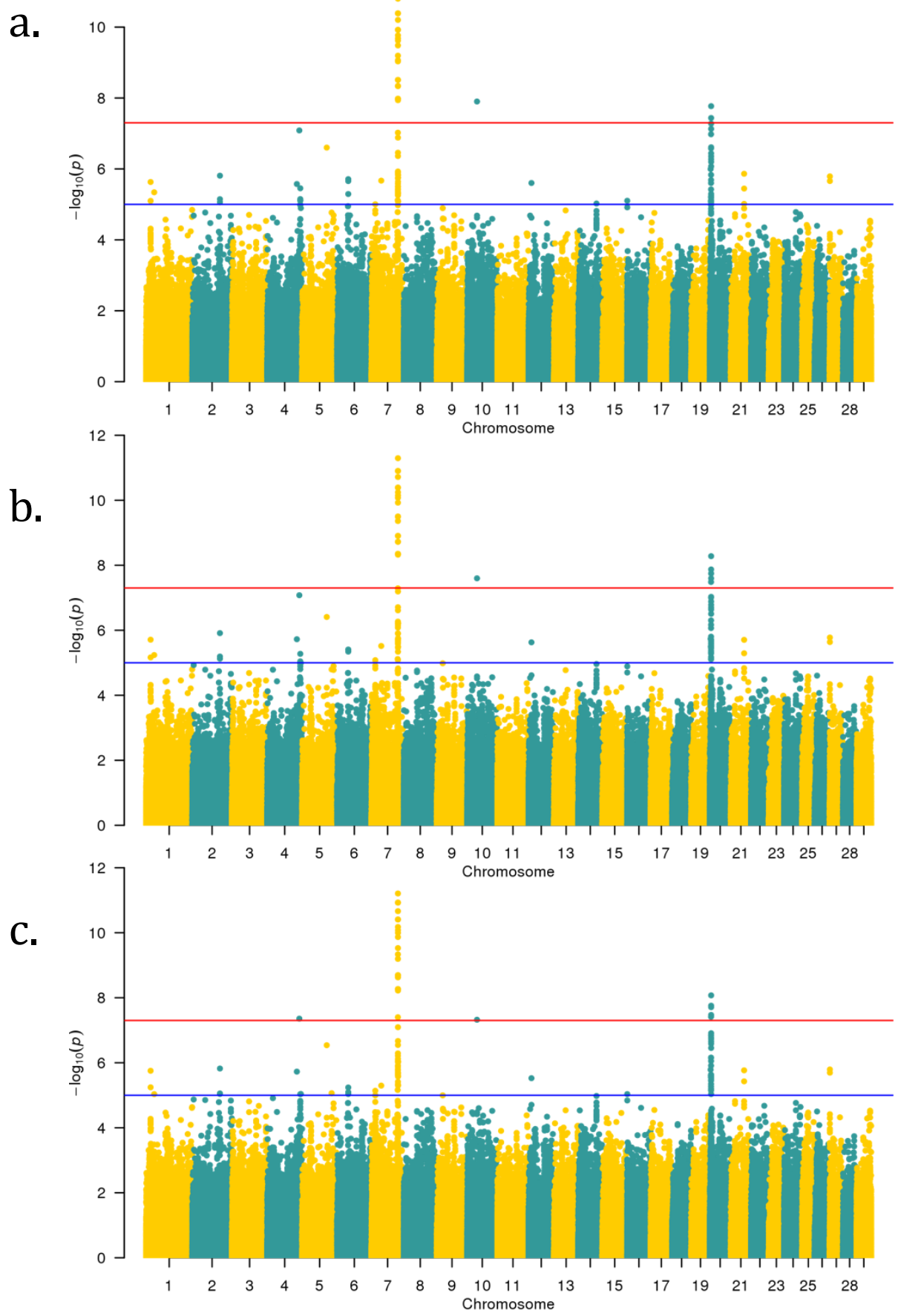

Figure 4.5. Manhattan Plots for Residual Feed Intake GWAS. (a.) Model 1 which analyzed contemporary group adjusted data. (b). Model 2, which included additive breed effects. (c). Model 3, which included additive breed effects and pairwise breed interactions to evaluate heterosis. 
a.

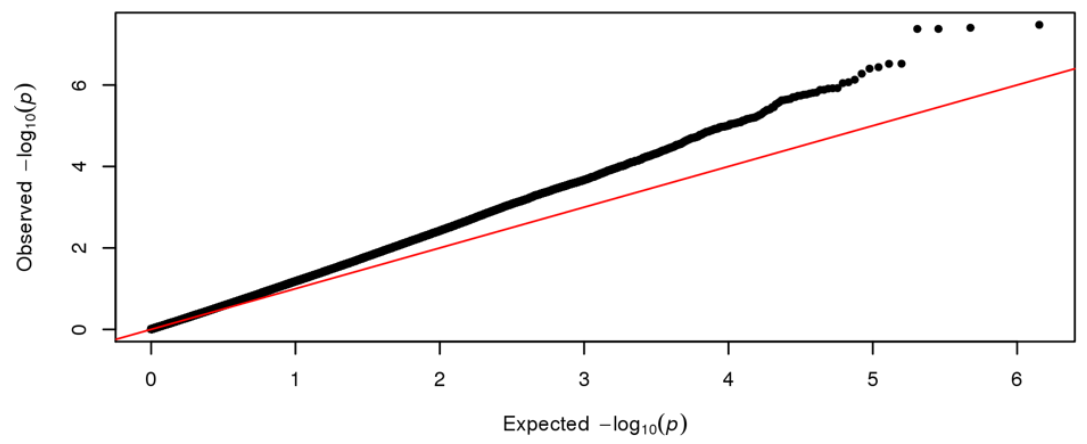

b.

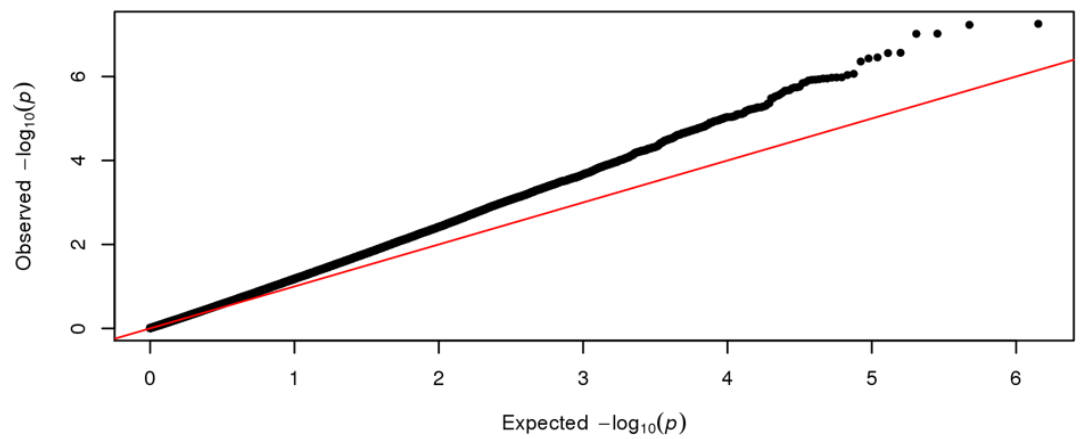

C.

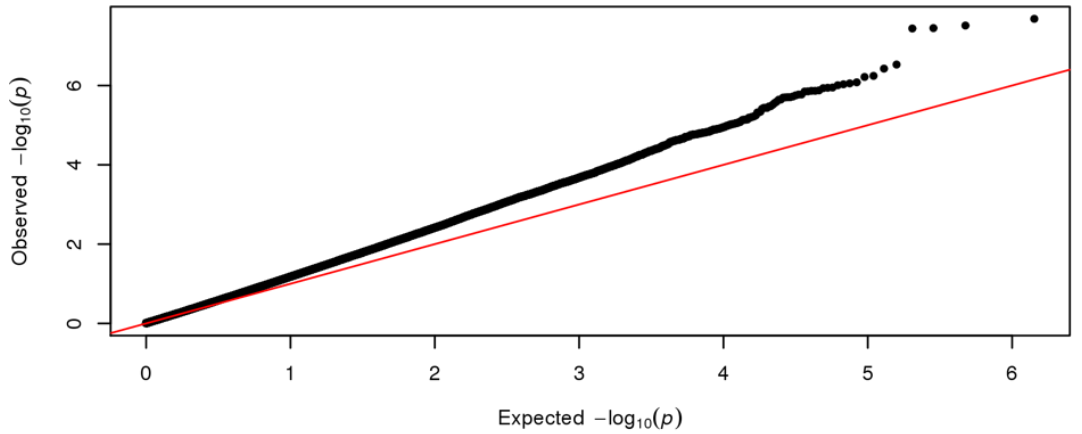

Figure 4.6. Q-Q Plot for Dry Matter Intake GWAS. (a.) Quantile-Quantile plots for Model 1 which analyzed traits preadjusted for contemporary group. (b). Model 2, which included additive breed effects. (c). Model 3, which included additive breed effects and pairwise breed interactions to evaluate heterosis. 
a.

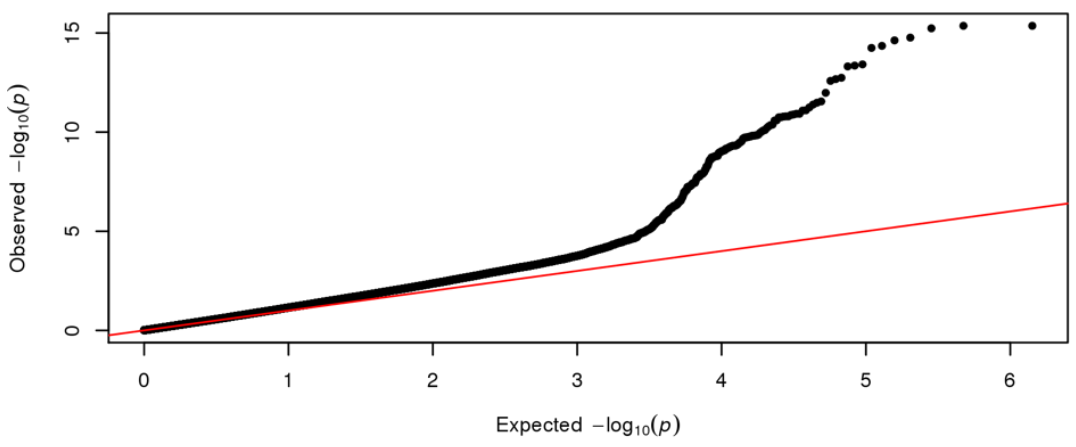

b.

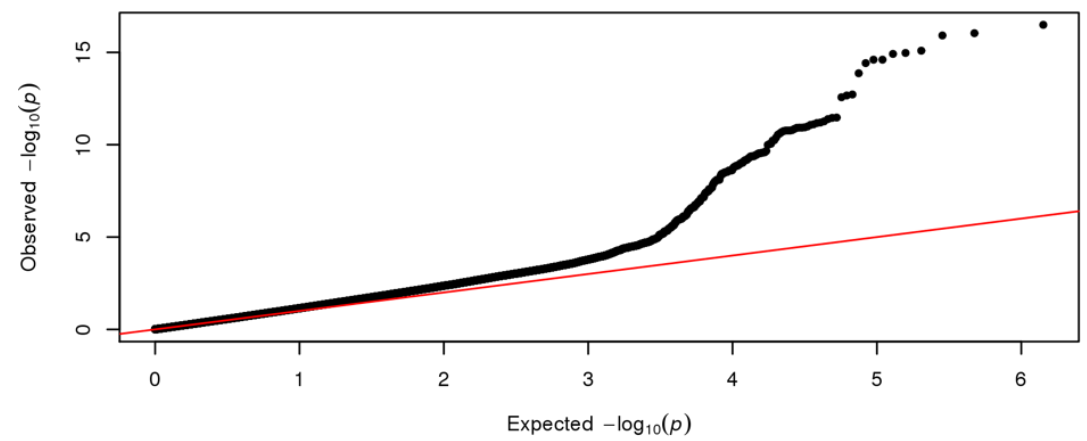

C.

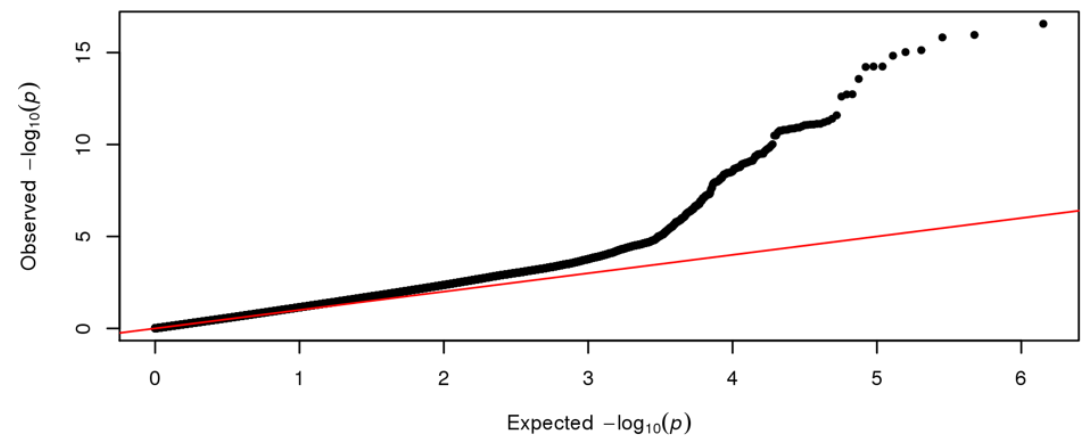

Figure 4.7. Q-Q Plot for Average Daily Gain GWAS. (a.) Quantile-Quantile plots for Model 1 which analyzed traits preadjusted for contemporary group. (b). Model 2, which included additive breed effects. (c). Model 3, which included additive breed effects and pairwise breed interactions to evaluate heterosis. 
a.

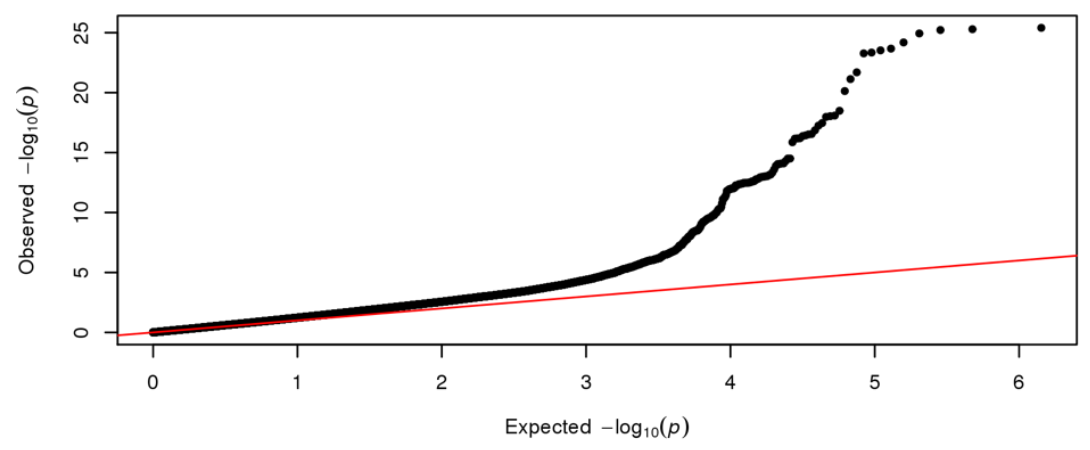

b.

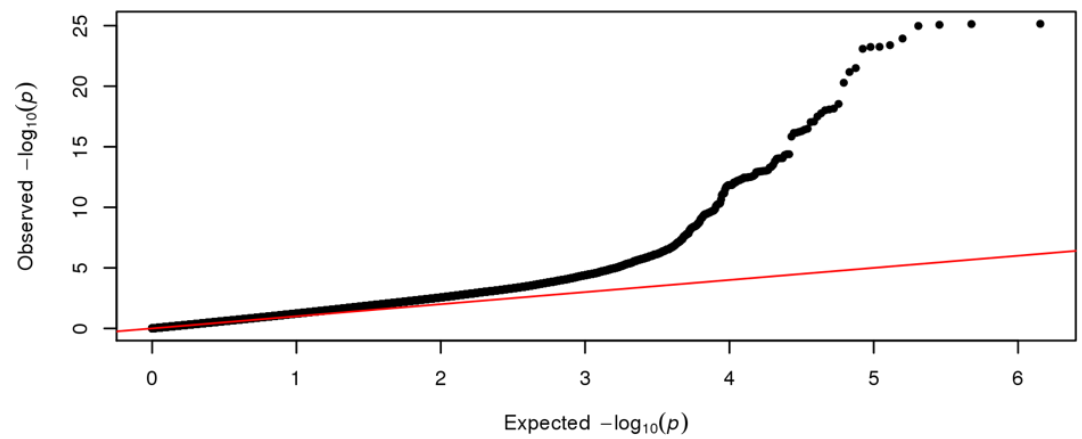

C.

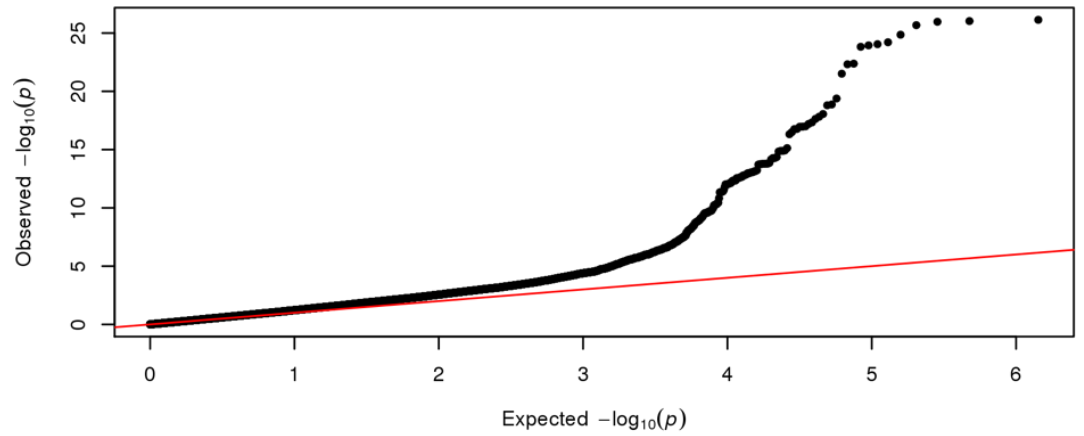

Figure 4.8. Q-Q Plot for Metabolic Mid-Weight GWAS. (a.) Quantile-Quantile plots for Model 1 which analyzed traits preadjusted for contemporary group. (b). Model 2, which included additive breed effects. (c). Model 3, which included additive breed effects and pairwise breed interactions to evaluate heterosis. 
a.

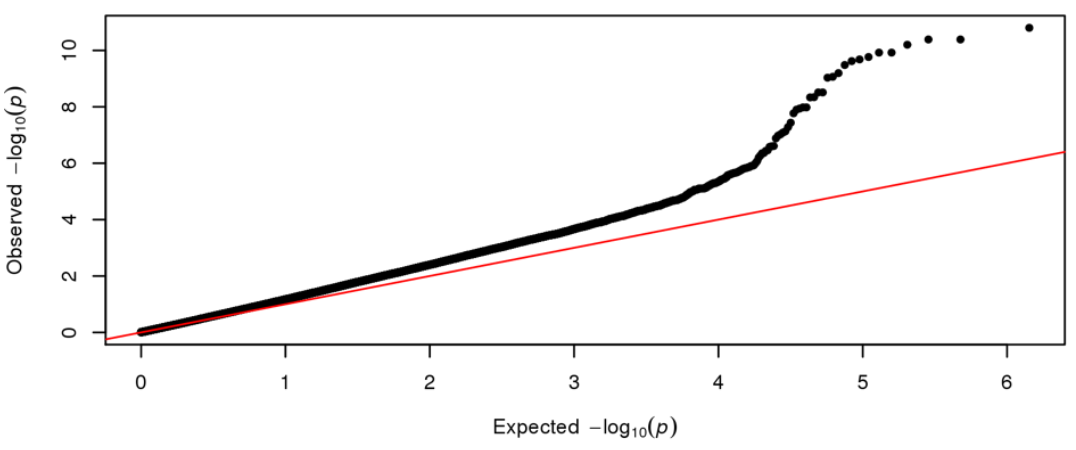

b.

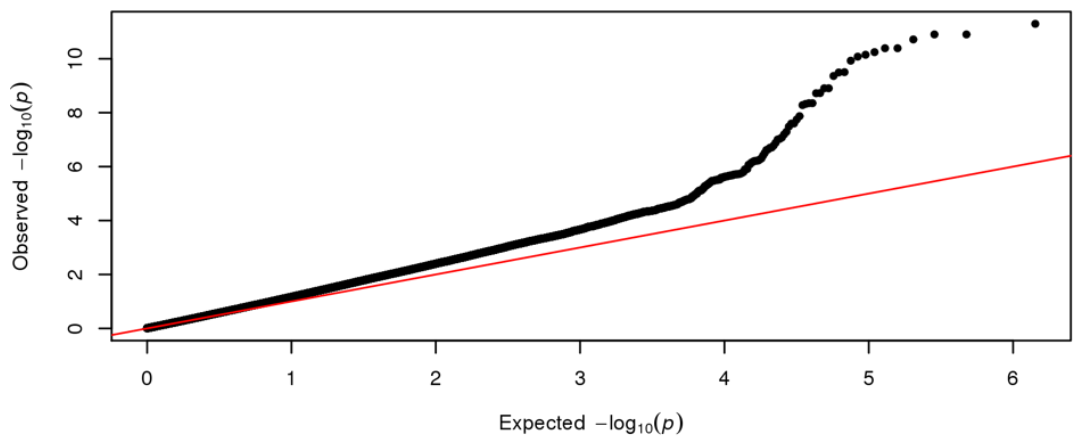

C.

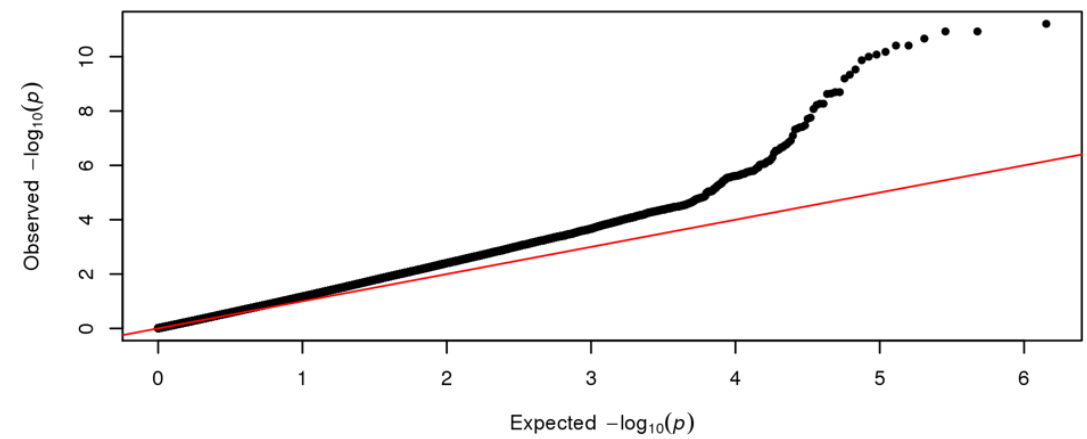

Figure 4.9. Q-Q Plot for Residual Feed Intake GWAS. (a.) Quantile-Quantile plots for Model 1 which analyzed traits preadjusted for contemporary group. (b). Model 2, which included additive breed effects. (c). Model 3, which included additive breed effects and pairwise breed interactions to evaluate heterosis. 


\section{Tables}

Table 4.1. Genotype data for Feed Efficiency animals by assay.

\begin{tabular}{ccc}
\hline Assay & No. of Variants & No. of Samples \\
\hline BOVGv1 & 47,843 & 1 \\
GGP-90KT & 76,999 & 2 \\
GGP-F250 & 227,234 & 4,463 \\
GGP-LDV3 & 26,504 & 40 \\
BovineHD & 777,962 & 1,638 \\
ICBF IDBV3 & 53,450 & 315 \\
BovineSNP50 & 58,336 & 7,018 \\
Total & & 13,477 \\
\hline
\end{tabular}


Table 4.2. Mean and Standard Deviations of CRUMBLER breed assignments for FE animals.

\begin{tabular}{|c|c|c|}
\hline Breed & $\begin{array}{c}\text { Average Ancestry } \\
(\%)\end{array}$ & $\begin{array}{c}\text { Standard Deviation } \\
(\%)\end{array}$ \\
\hline Angus & 31.87 & \pm 24.67 \\
\hline Braunvieh & 1.60 & \pm 4.13 \\
\hline Brown Swiss & 0.30 & \pm 1.47 \\
\hline Charolais & 6.91 & \pm 13.66 \\
\hline Gelbvieh & 6.69 & \pm 13.64 \\
\hline Guernsey & 0.56 & \pm 2.01 \\
\hline Hereford & 17.39 & \pm 29.19 \\
\hline Holstein & 2.03 & \pm 3.79 \\
\hline Indicine & 0.17 & \pm 1.56 \\
\hline Japanese Black & 0.22 & \pm 4.34 \\
\hline Jersey & 0.26 & \pm 1.40 \\
\hline Limousin & 3.08 & \pm 11.40 \\
\hline Ndama & 0.00 & \pm 0.17 \\
\hline Red Angus & 16.02 & \pm 20.06 \\
\hline Romagnola & 0.19 & \pm 1.20 \\
\hline Shorthorn & 3.70 & \pm 4.90 \\
\hline Simmental & 9.01 & \pm 14.51 \\
\hline
\end{tabular}


Table 4.3. Significance between models using likelihood ratio tests.

\begin{tabular}{ccc}
\hline & Model 1 vs. Model 2 & Model 2 vs. Model 3 \\
\hline ADG & 0.013911 & 0.002496 \\
DMI & 0.003485 & 0.002338 \\
MMWT & 0.035999 & 0.000000 \\
RFI & 0.020166 & 0.053308 \\
\hline
\end{tabular}


Table 4.4. Variance component and pseudo-heritability estimates for feed efficiency and component traits.

\begin{tabular}{cccc}
\hline Model : Trait & $\mathbf{h}^{2}$ & Va & Ve \\
\hline Model 1 : RFI & 0.45 & 2.3029 & 2.7901 \\
Model 2 : RFI & 0.45 & 2.2823 & 2.7921 \\
Model 3 : RFI & 0.45 & 2.2937 & 2.7860 \\
Model 1 : MMWT & 0.56 & 112.7300 & 88.3340 \\
Model 2 : MMWT & 0.56 & 111.1450 & 88.5959 \\
Model 3 : MMWT & 0.56 & 112.3270 & 87.6046 \\
Model 1 : DMI & 0.50 & 5.1659 & 5.0817 \\
Model 2 : DMI & 0.50 & 5.0345 & 5.1036 \\
Model 3 : DMI & 0.50 & 5.0048 & 5.0977 \\
Model 1 : ADG & 0.42 & 0.1796 & 0.2462 \\
Model 2 : ADG & 0.41 & 0.1741 & 0.2472 \\
Model 3 : ADG & 0.41 & 0.1727 & 0.2470 \\
\hline
\end{tabular}




\section{Supplementary Materials}

\section{Chapter 2 Supplementary Information}

EIGENSOFT Source Code Edits

EIGENSOFT packages CONVERTF and SMARTPCA are required for use by SNPweights. However, SMARTPCA within versions of EIGENSOFT beyond 5.0.2 are not compatible with SNPweights. To establish compatibility, the following edits must be made to the SMARTPCA source code:

1. Download EIGENSOFT from https://github.com/DReichLab/EIG/

2. Go to directory /src/eigensrc/

3. Open smartpca.c

4. Find the string: printf("trace: \%9.3f $\backslash n ", y)$;

5. Remove the comment characters (") to make the code an active line. In version 7.2.1, the code is located at line \#1079. In version 6.1.4, the code is at line \#1138. The SMARTPCA program still calculates trace, changing the code outputs the value of trace for use by SNPweights software.

To recompile the modified code, follow the instructions of the EIGENSOFT authors. This information is found in the README file in the EIGENSOFT download base directory. 


\section{Chapter 2 Supplementary Methods}

\section{Preliminary fastSTRUCTURE analyses conducted on subsets of breeds.}

Angus vs Simmental (Fig. S2.2)

FastSTRUCTURE analysis was conducted for 5,552 registered Angus and 15,858 registered Simmental. To reduce the potential effects of unequal sample sizes, three analyses were performed. Each analysis included all 5,552 registered Angus and 5,286 of the registered Simmental. In total, 4,390 Angus were predicted to have at least $97 \%$ Angus ancestry in all three analyses and 1,583 Simmental were predicted to have at least $97 \%$ Simmental ancestry and were retained for further analysis.

Angus vs Gelbvieh (Fig. S2.3)

FastSTRUCTURE analysis was conducted for 5,552 registered Angus and 12,835 registered Gelbvieh. Three analyses were performed including all 5,552 registered Angus and 4,279, 4,278, and 4,278 registered Gelbvieh, respectively. In total, 4,351 Angus were predicted to have at least $97 \%$ Angus ancestry in all three analyses and 6,000 Gelbvieh were predicted to have at least $97 \%$ Gelbvieh ancestry and were retained for further analysis.

Angus vs Limousin (Fig. S2.4)

FastSTRUCTURE analysis was conducted for the 4,268 registered Angus identified as having at least $97 \%$ Angus ancestry in both the Angus vs Simmental and Angus vs Gelbvieh analyses and 2,734 registered Limousin. In total, 1,470 
Angus were predicted to have at least $97 \%$ Angus ancestry and 367 Limousin were predicted to have at least $97 \%$ Limousin ancestry and were retained for further analysis.

Angus vs Red Angus (Fig. S2.5)

FastSTRUCTURE analysis was conducted for the 4,268 registered Angus identified as having at least $97 \%$ Angus ancestry in both the Angus vs Simmental and Angus vs Gelbvieh analyses and 1,377 registered Red Angus. In total, 508 Angus were predicted to have at least $97 \%$ Angus ancestry and 124 Red Angus were predicted to have at least $97 \%$ Red Angus ancestry and were retained for further analysis.

Red Angus vs Hereford vs Shorthorn vs Salers (Fig. S2.6) FastSTRUCTURE analysis was conducted for the 1,377 registered Red Angus, 969 registered Hereford, 291 registered Shorthorn, and 68 registered Salers. In total, 700 Red Angus were predicted to have at least 97\% Angus ancestry, 335 Hereford were predicted to have at least $97 \%$ Hereford ancestry, 212 Shorthorn were predicted to have at least $97 \%$ Shorthorn ancestry, and 0 Salers were predicted to have at least $97 \%$ Salers ancestry and were retained for further analysis. The Salers was then removed as a reference population. 
Red Angus vs Hereford vs Shorthorn (Fig. S2.7)

FastSTRUCTURE analysis was conducted for the 1,377 registered Red Angus, 969 registered Hereford and 291 registered Shorthorn. In total, 518 Red Angus were predicted to have at least $97 \%$ Angus ancestry, 348 Hereford were predicted to have at least $97 \%$ Hereford ancestry, and 166 Shorthorn were predicted to have at least $97 \%$ Shorthorn ancestry, and were retained for further analysis.

N'Dama vs Nelore vs Brahman (Fig. S2.8)

FastSTRUCTURE analysis was conducted for the 98 N'Dama, 941Nelore, and 127 registered Brahman. In total, 59 N'Dama were predicted to have at least 97\% N'Dama ancestry, 86 Brahman were predicted to have at least $97 \%$ Brahman ancestry, and 708 Nelore were predicted to have at least $97 \%$ Nelore ancestry, and were retained for further analysis. 


\section{Chapter 2 Supplementary Figures}

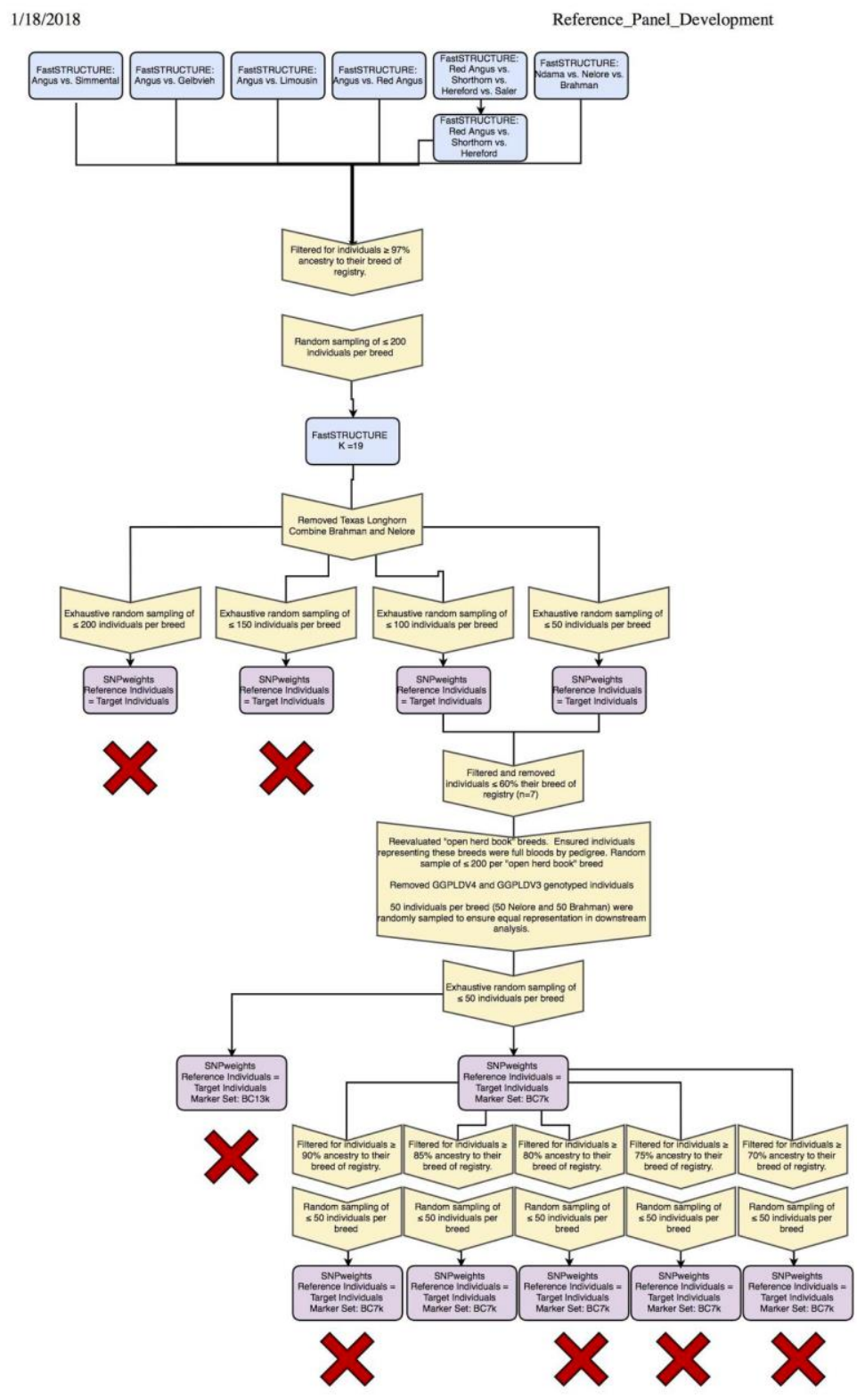

Figure S2.1. An overview of the processes and iterations of filtering conducted in the development of the reference panel. Blue = FastSTRUCTURE analyses, Purple $=$ SNPweights analyses, Yellow $=$ Data management processes . 

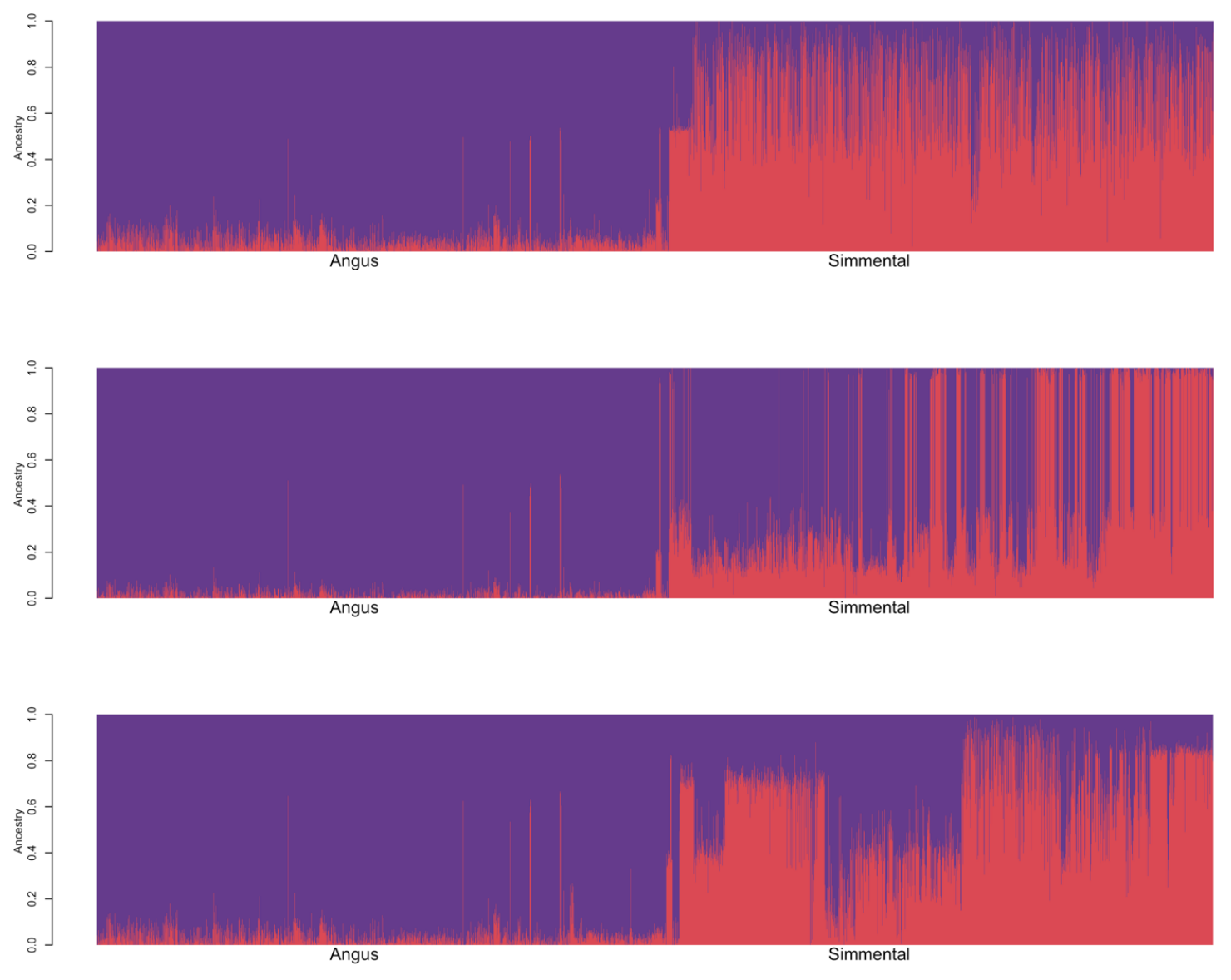

Figure S2.2. Preliminary FastSTRUCTURE analysis of candidate Angus and Simmental reference population animals. Three analyses were run to compare equal numbers of animals from each breed when the available sample sizes for each breed differed. Each row represents an analysis and each animal is represented as a vertical line. 

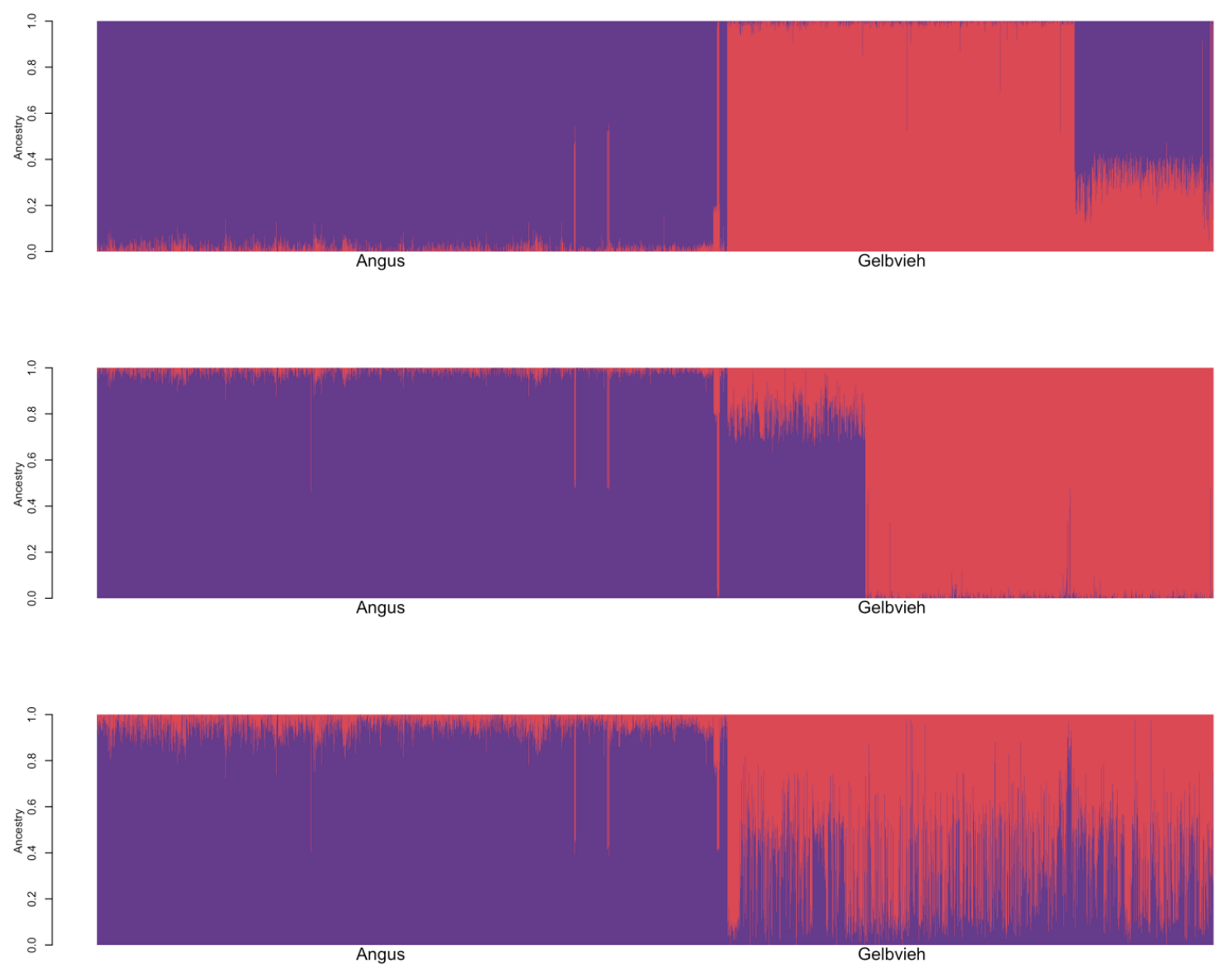

Figure S2.3. Preliminary FastSTRUCTURE analysis of candidate Angus and Gelbvieh reference population animals. Three analyses were run to compare equal numbers of animals from each breed when the available sample sizes for each breed differed. Each row represents an analysis and each animal is represented as a vertical line. 


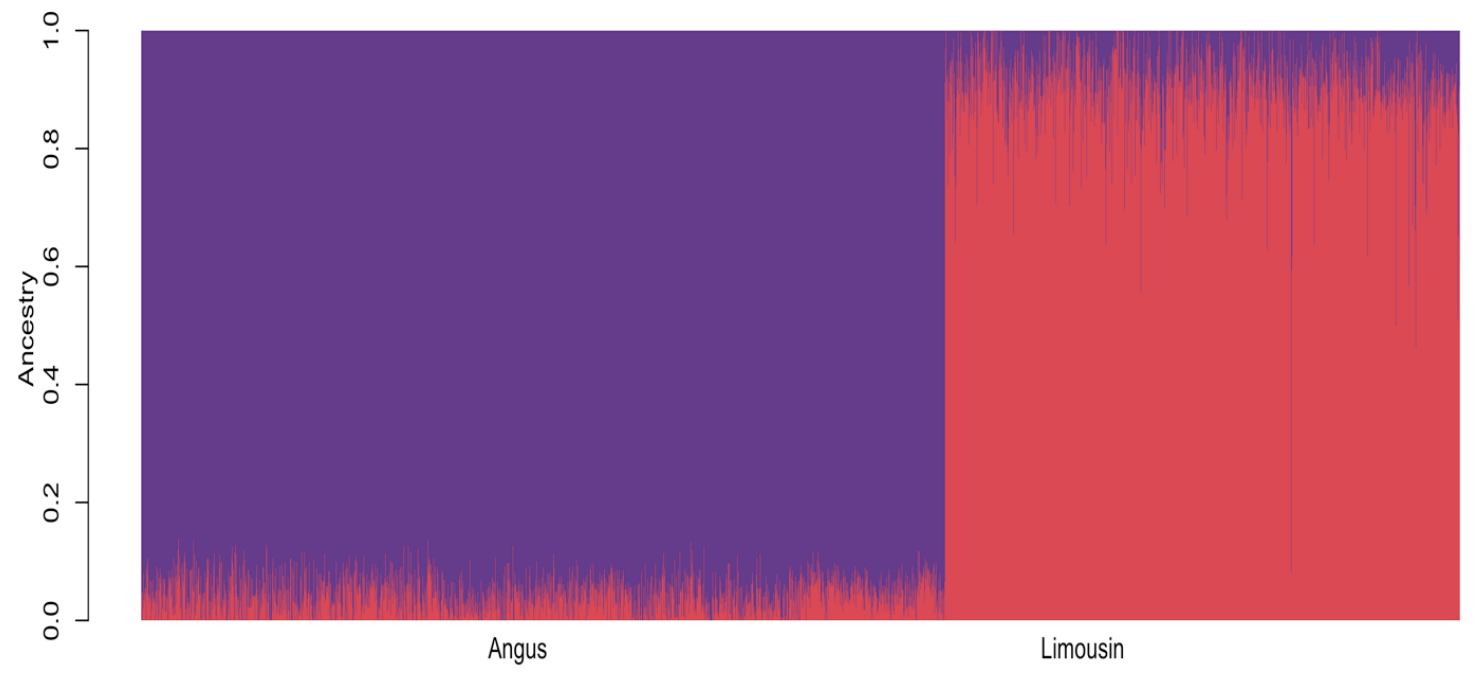

Figure S2.4. Preliminary FastSTRUCTURE analysis of candidate Angus and Limousin reference population animals. Each animal is represented as a vertical line. 


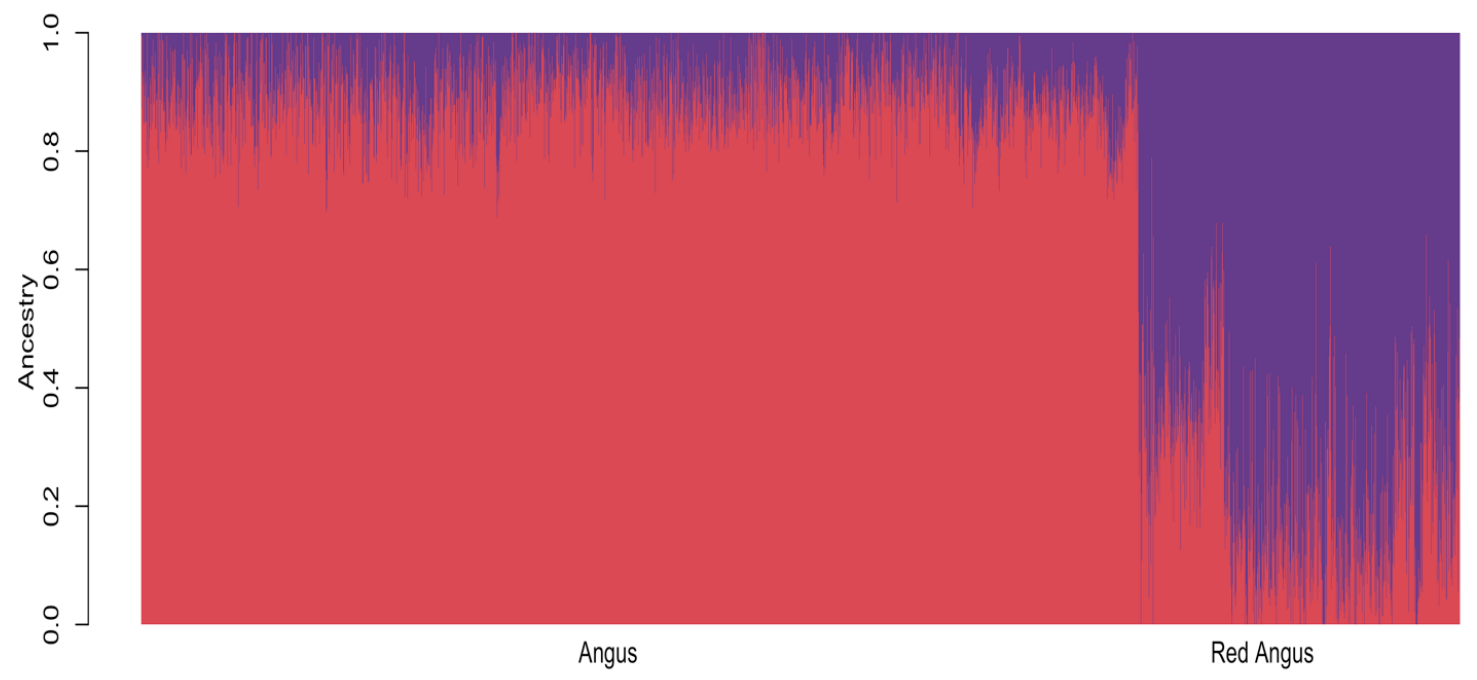

Figure S2.5. Preliminary FastSTRUCTURE analysis of candidate Angus and Red Angus reference population animals. Each animal is represented as a vertical line. 


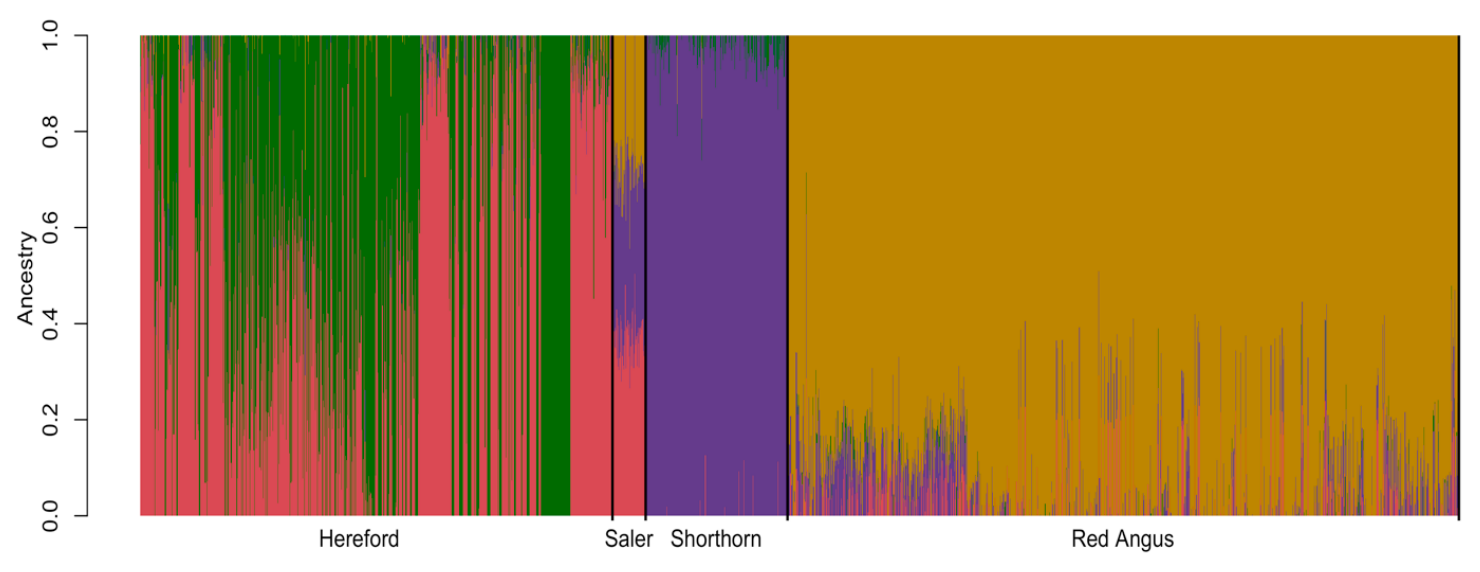

Figure S2.6. Preliminary FastSTRUCTURE analysis of candidate Red Angus, Hereford, Shorthorn, and Salers reference population animals. Each animal is represented as a vertical line. 


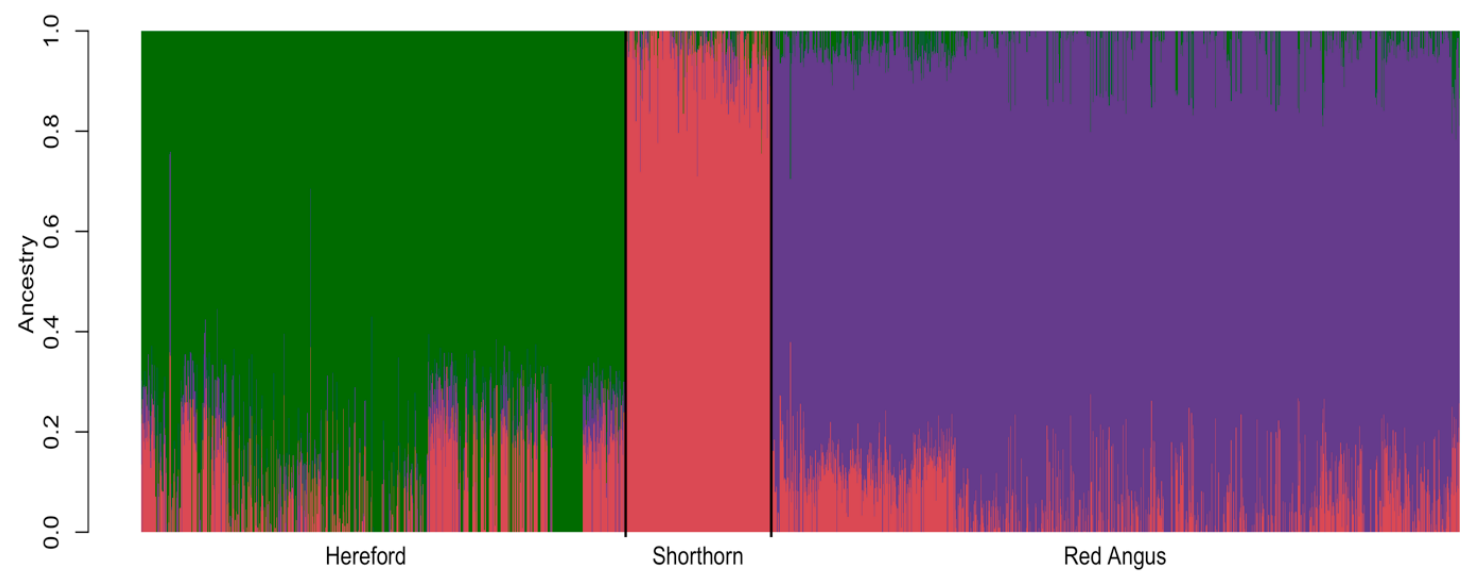

Figure S2.7. Preliminary FastSTRUCTURE analysis of candidate Red Angus, Hereford, and Shorthorn reference population individuals. Each animal is represented as a vertical line. 


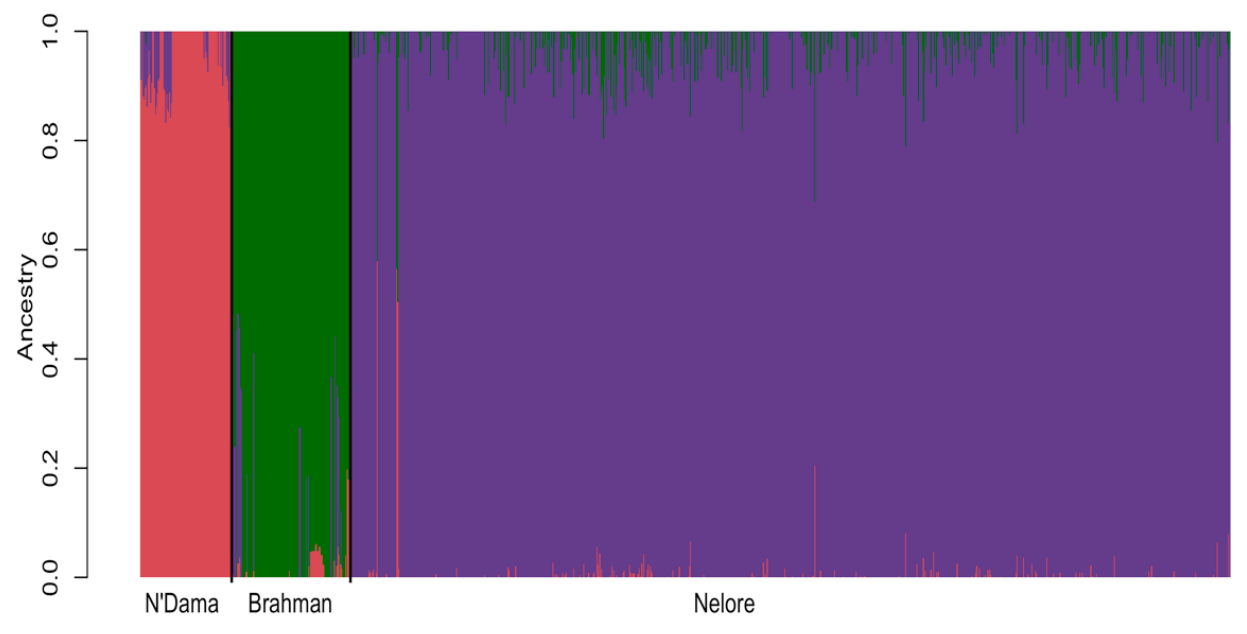

Figure S2.8. FastSTRUCTURE analysis of candidate N'Dama, Brahman and Nelore reference population individuals. Each animal is represented as a vertical line. 

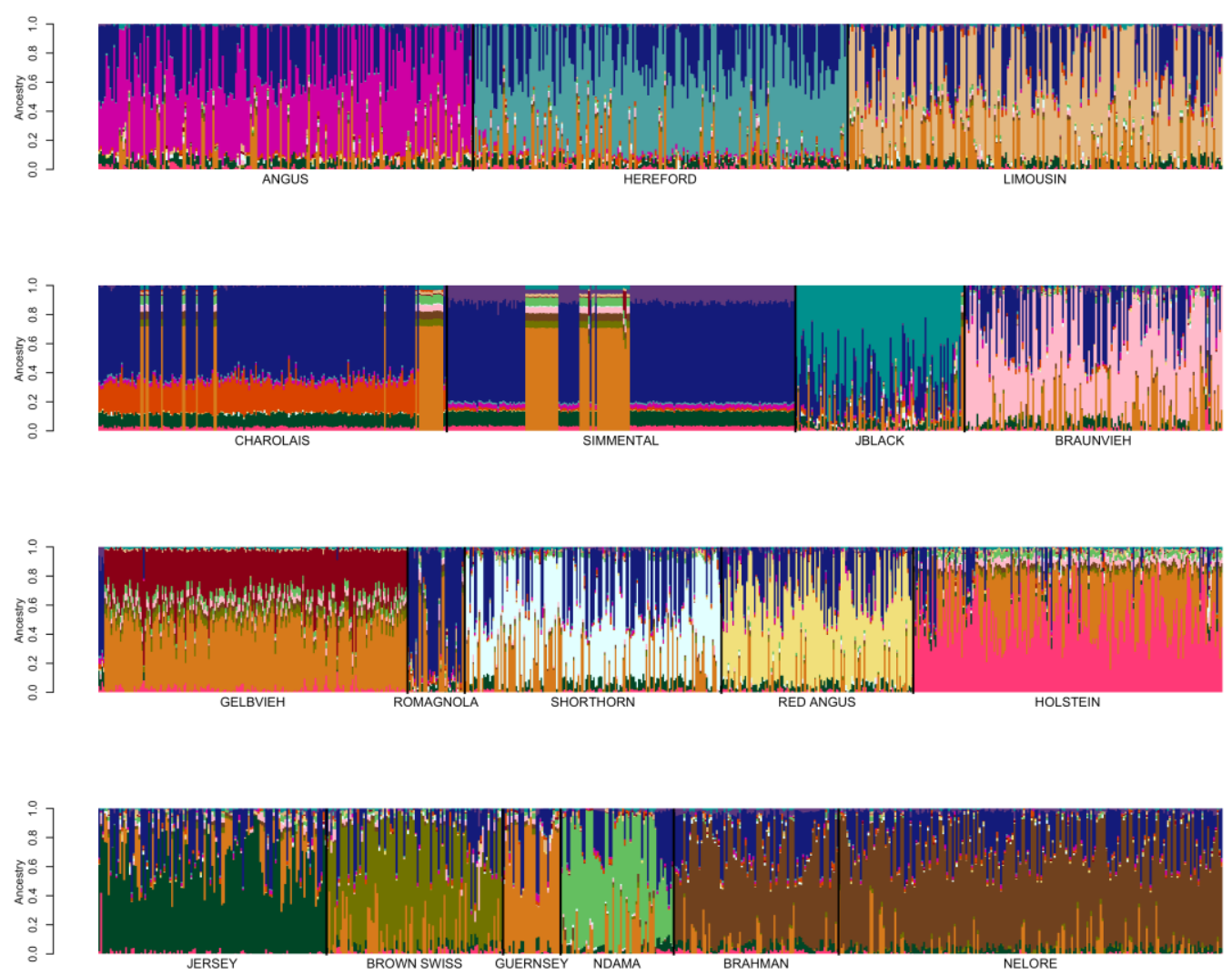

Figure S2.9. SNPweights self-assignment analysis for the reference sample set containing $\leq \mathbf{2 0 0}$ individuals per breed analyzed using the BC7K marker set. 

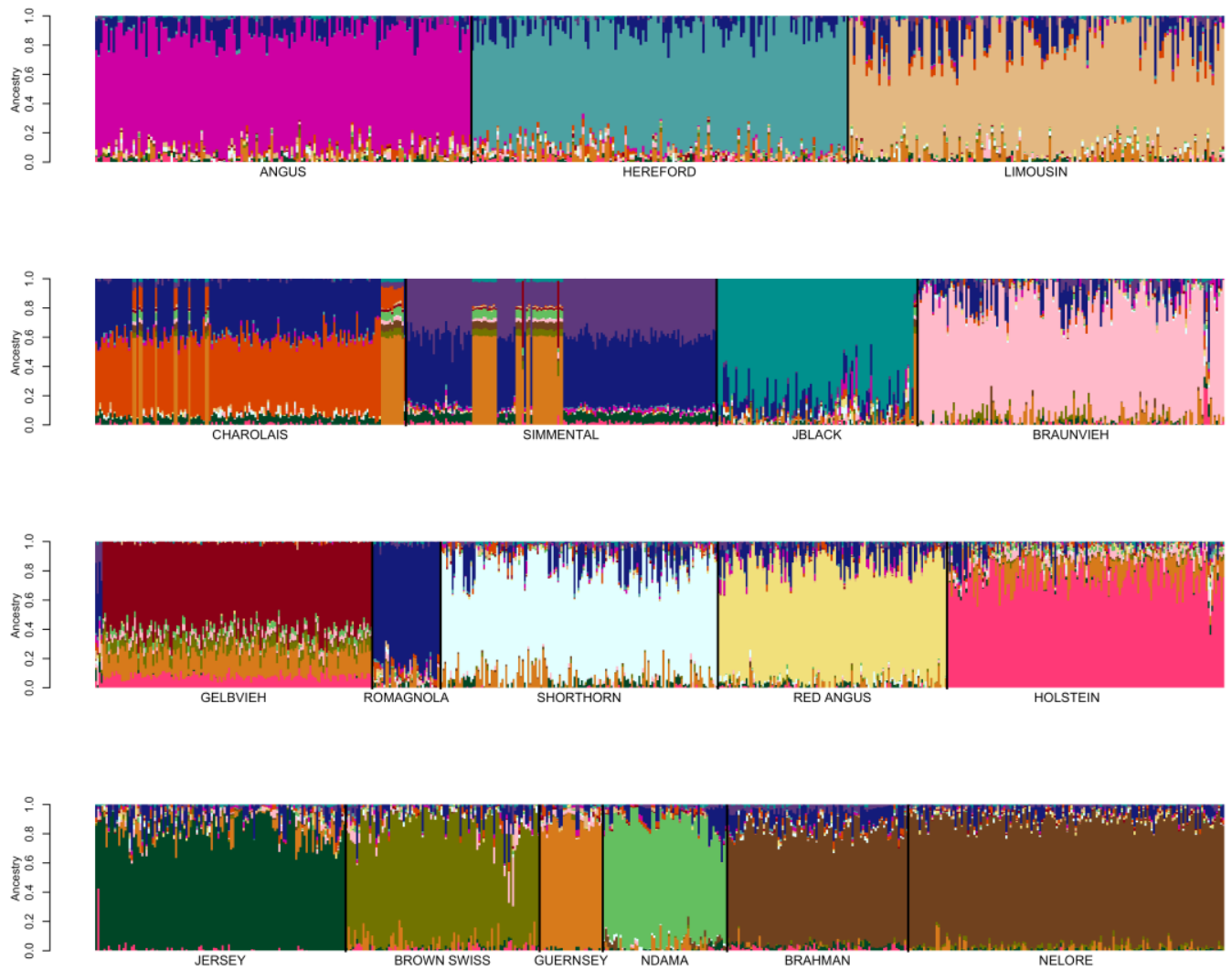

Figure S2.10. SNPweights self-assignment analysis for the reference sample set containing $\leq 150$ individuals per breed analyzed using the $\mathrm{BC} 7 \mathrm{~K}$ marker set. 


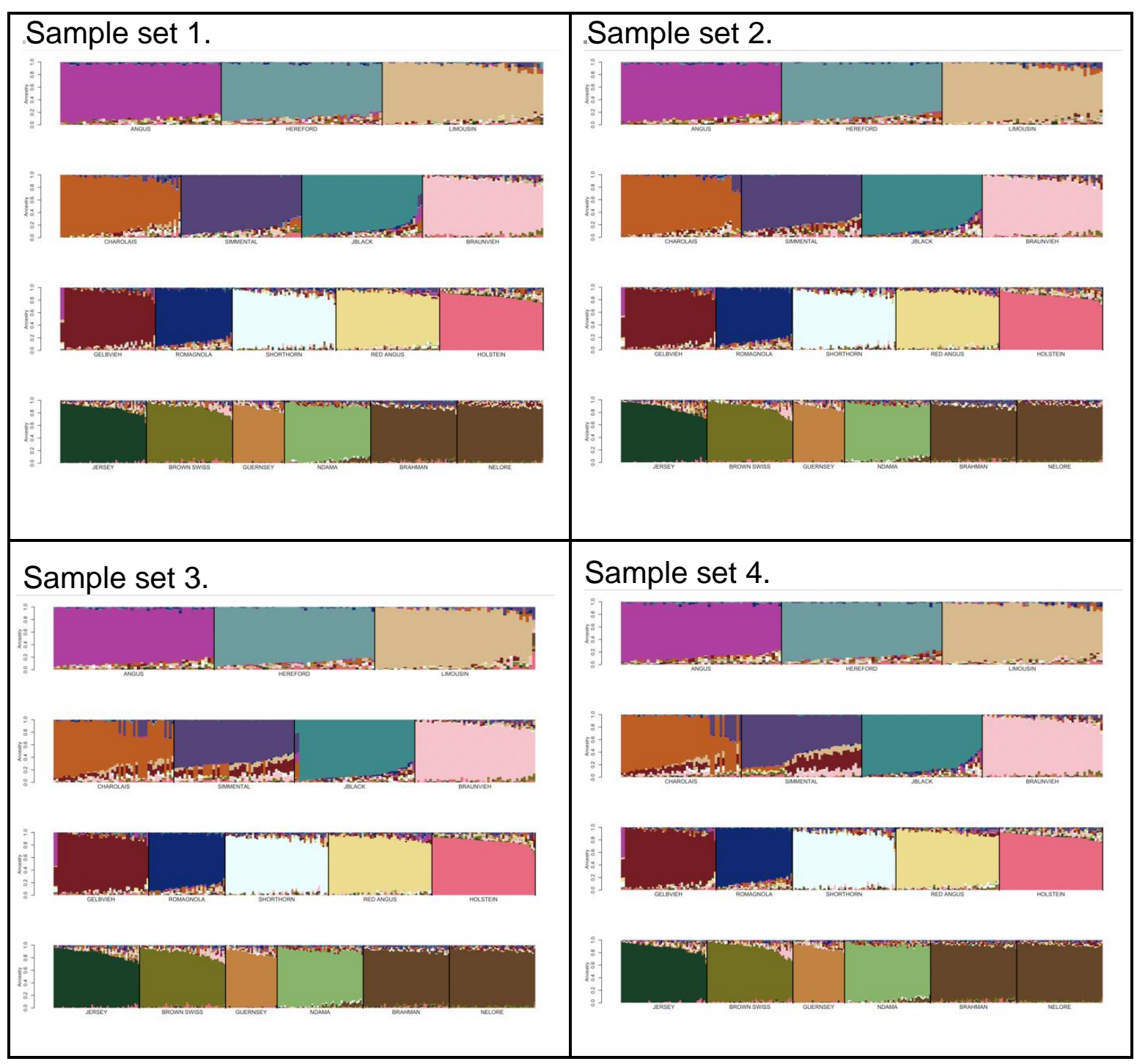

Figure S2.11. SNPweights self-assignment analysis for the reference sample set containing $\leq \mathbf{5 0}$ individuals per breed analyzed using the $\mathrm{BC} 7 \mathrm{~K}$ marker set. Reference breed panels were constructed by randomly sampling $\leq 50$ individuals per breed until all individuals were represented in at least one set, resulting in 5 candidate reference sample sets (sample set 5 is shown in Figure 2.4a). 


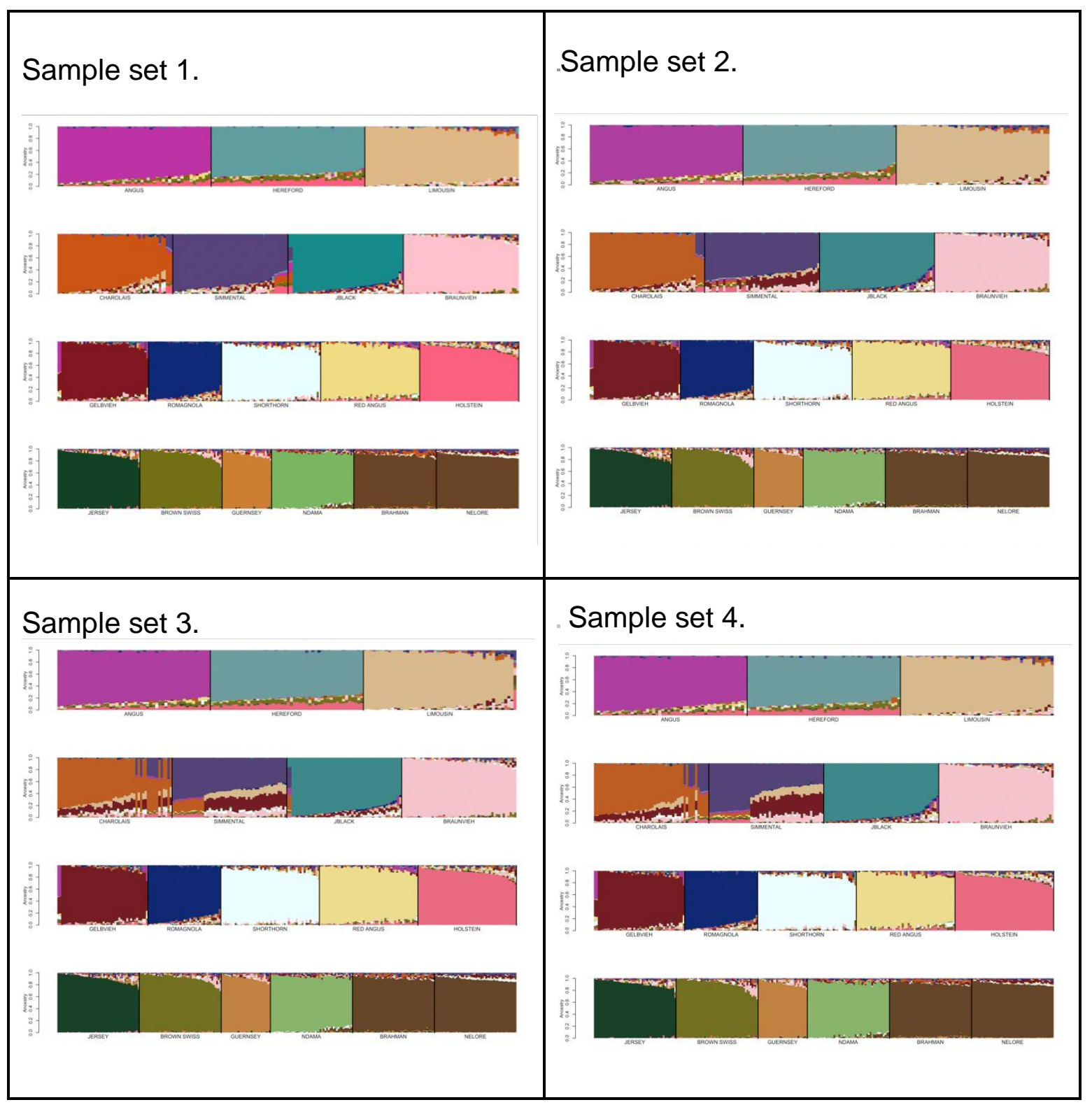

Figure S2.12. SNPweights self-assignment analysis for the reference sample set containing $\leq \mathbf{5 0}$ individuals per breed analyzed using the $\mathrm{BC} 13 \mathrm{~K}$ marker set. Reference breed panels were constructed by randomly sampling $\leq 50$ individuals per breed until all individuals were represented in at least one set, resulting in 5 candidate reference sample sets (sample set 5 is shown in Figure 2.4b). 

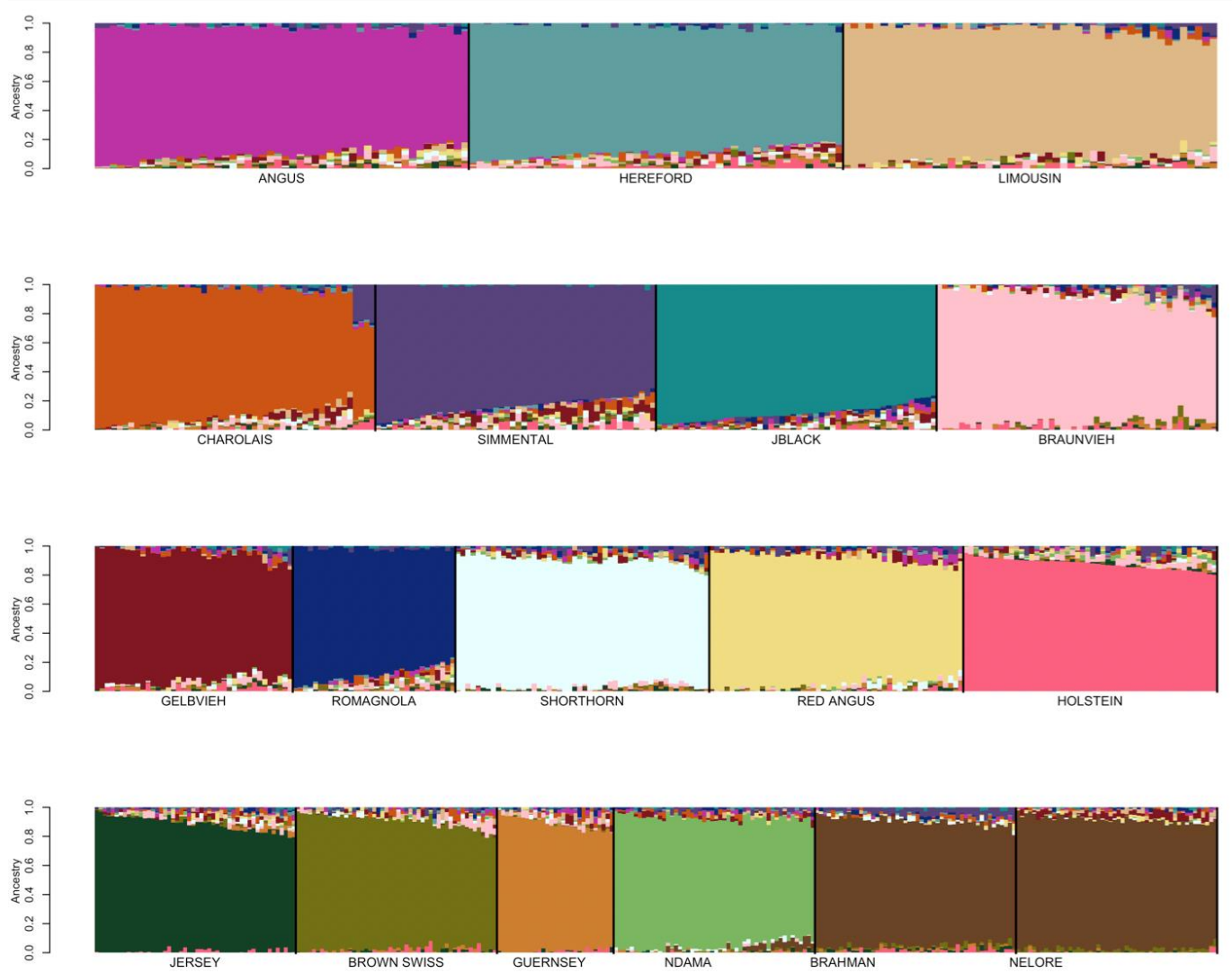

Figure S2.13. SNPweights self-assignment analysis for the reference sample set with $\geq 80 \%$ ancestry to breed of registry and $\leq 50$ individuals per breed using the BC7K marker set. 

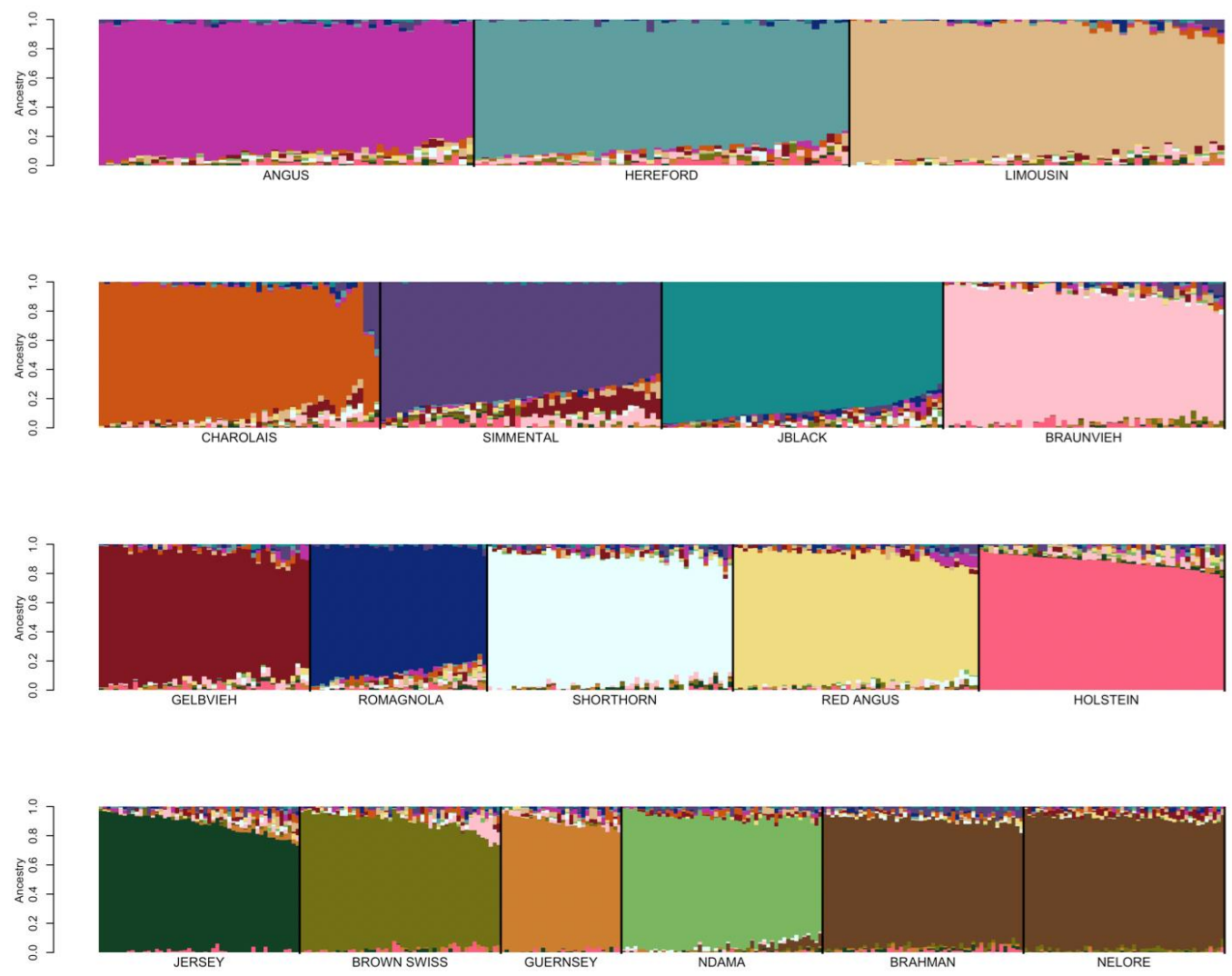

Figure S2.14. SNPweights self-assignment analysis for the reference sample set with $\geq 75 \%$ ancestry to breed of registry and $\leq 50$ individuals per breed using the BC7K marker set. 

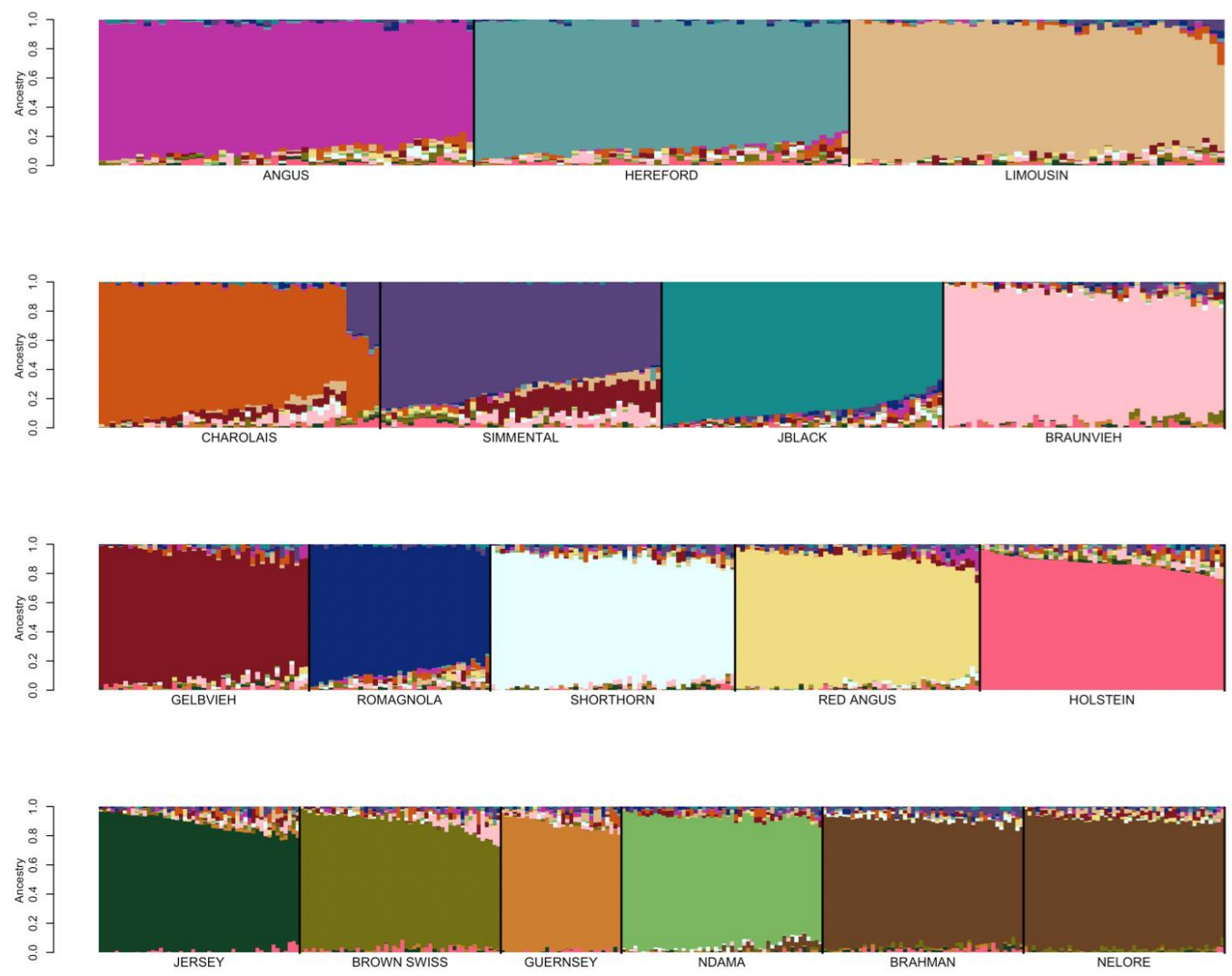

Figure S2.15. SNPweights self-assignment analysis for the reference sample set with $\geq 70 \%$ ancestry to breed of registry and $\leq 50$ individuals per breed using the BC7K marker set. 
a.
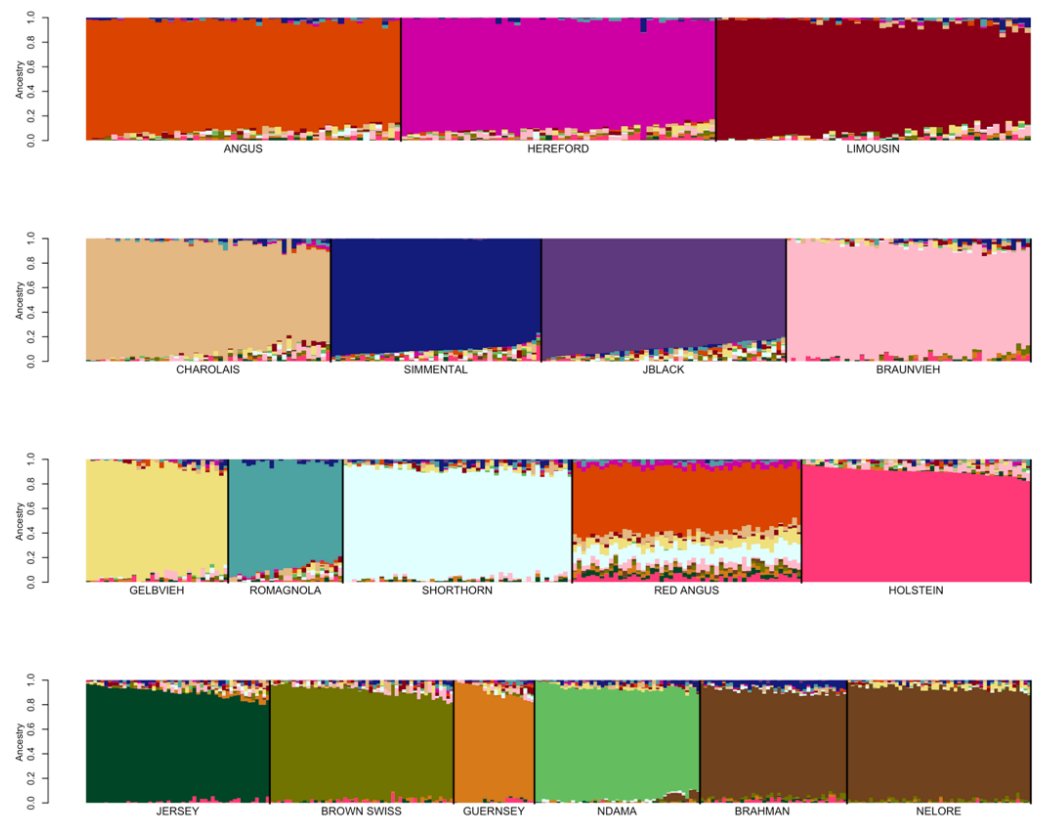

b.
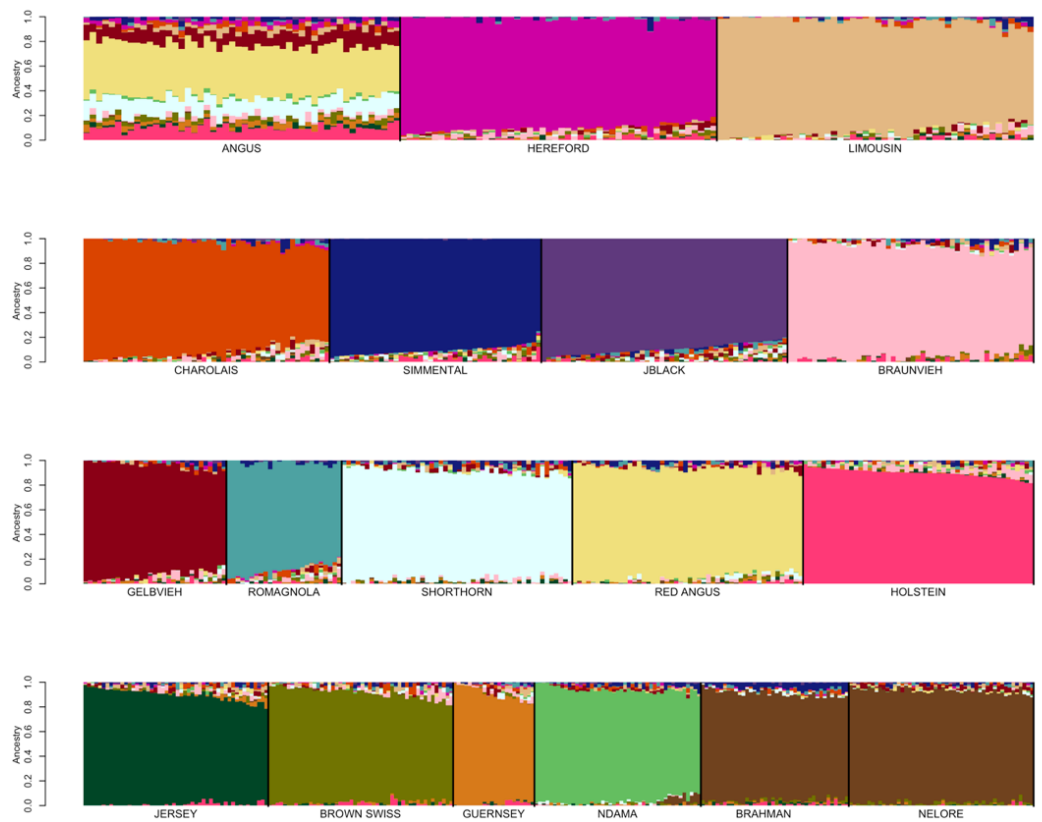

Figure S2.16. SNPweights self-assignment analyses using a reference panel with $\leq 50$ individuals per breed and sampling from the individuals with $\geq 85 \%$ assignment to their breed of registry but with (a) Red Angus or (b) Angus excluded from the reference panel. The Red Angus and Angus individuals in the reference panel were retained for ancestry estimation. 


\section{Chapter 3 Supplementary Figures}

a
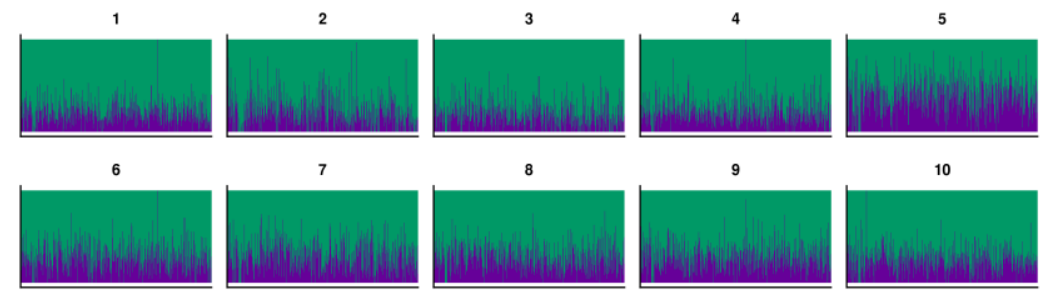

8

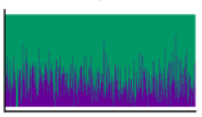

9

10

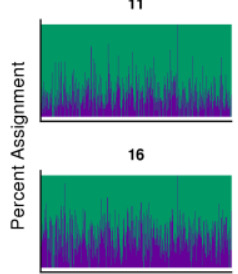

12

13

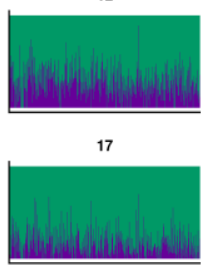

22

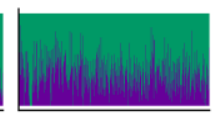

18

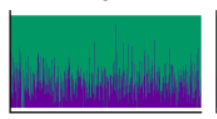

14

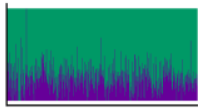

15

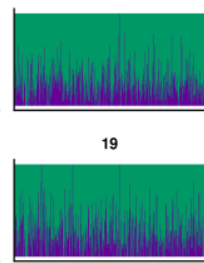

24

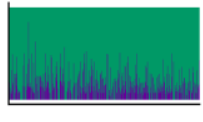

20
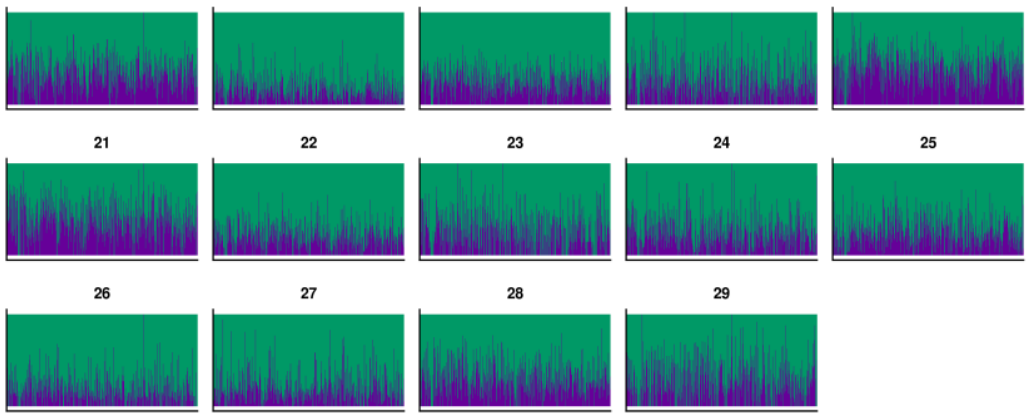

Individual

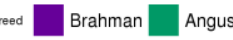

b

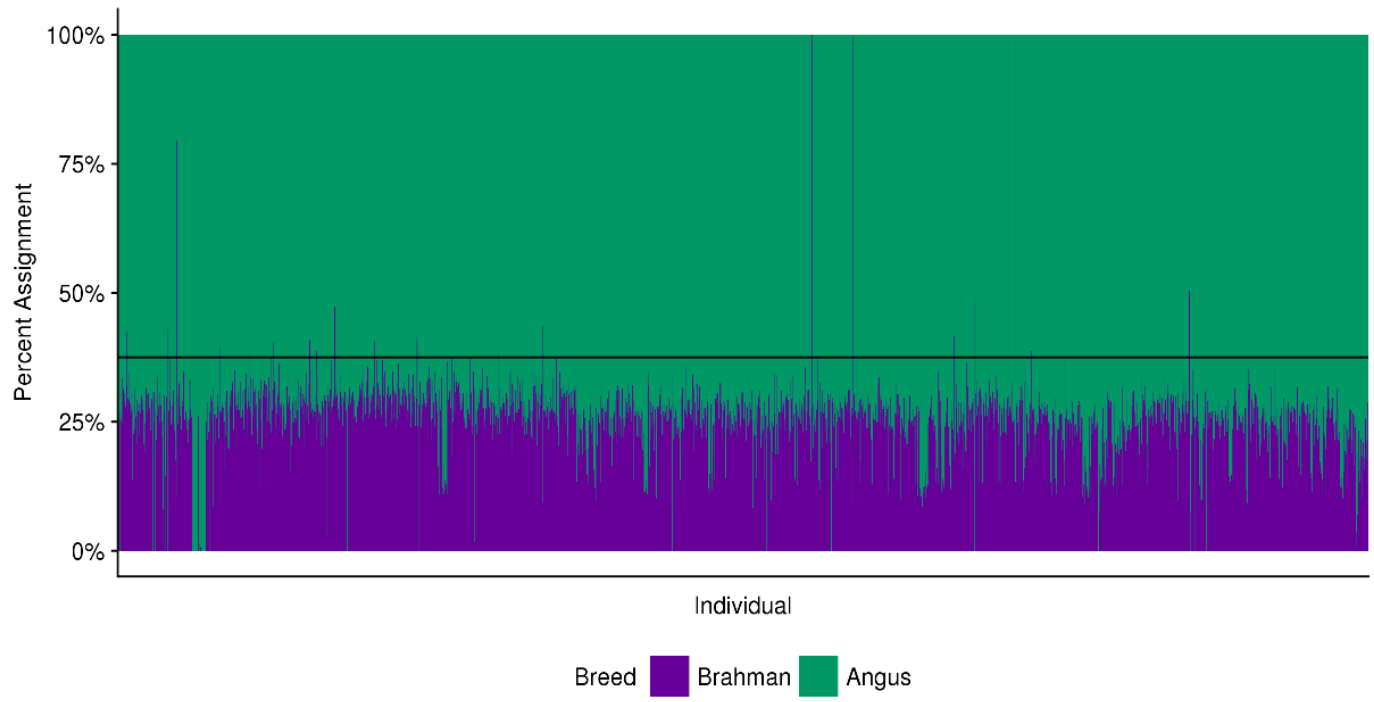

Figure S3.1. Brangus Ancestry Estimation by Individual (Prior to

Outlier Removal). (a.) Plot of RFmix global ancestry assignment for Brangus individuals by chromosome. Individuals are represented by vertical bars. (b.) Plot of RFmix global ancestry assignment for Brangus individuals genome-wide. Individuals are represented by vertical bars. The black line represents the expected proportion of Brahman (3/8) based on the foundation of the Brangus breed. 
a.
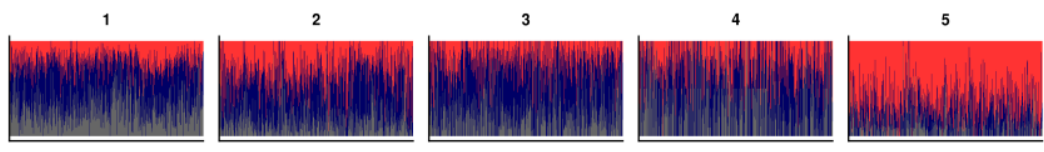

6

7
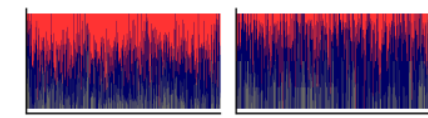

8

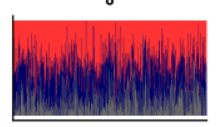

9

10

11

12
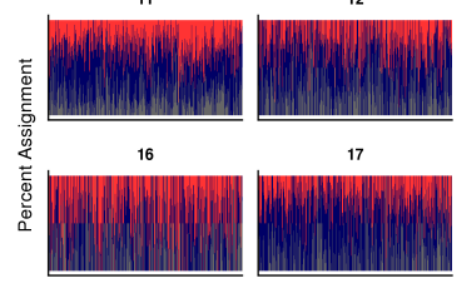

13
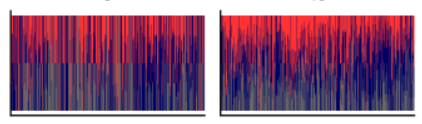

14

15

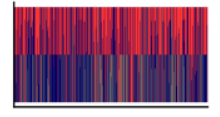

18

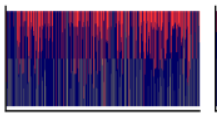

19

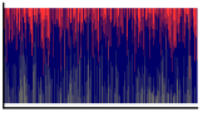

20

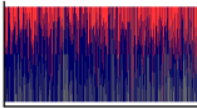

22

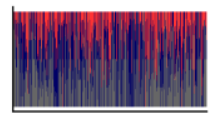

23

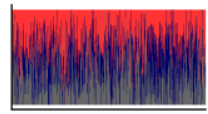

24

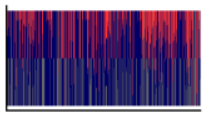

25
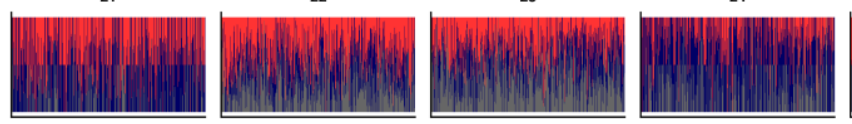

29

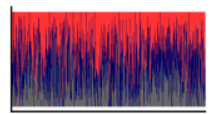

27

28
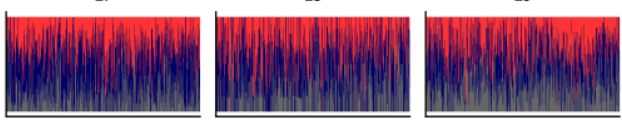

Individual

Breed

Brahman

Hereford

Shorthorn

b

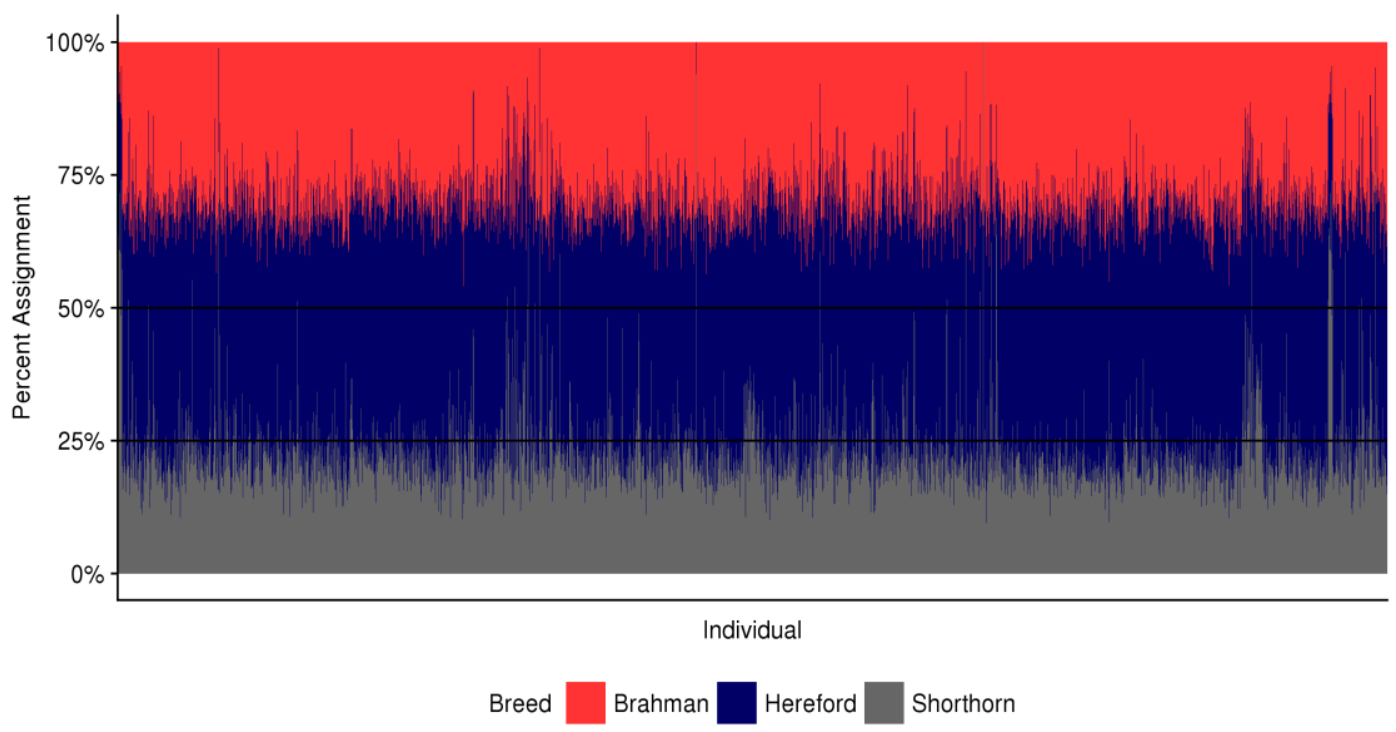

Figure S3.2. Beefmaster Ancestry Estimation by Individual (Prior to Outlier Removal). (a.) Plot of RFmix global ancestry assignment for Beefmaster individuals by chromosome. Individuals are represented by vertical bars. (b.) Plot of RFmix global ancestry assignment for Beefmaster individuals genome-wide. Individuals are represented by vertical bars. The black lines represent the expected proportions of Shorthorn (1/4) and Hereford (1/4)based on the foundation of the 

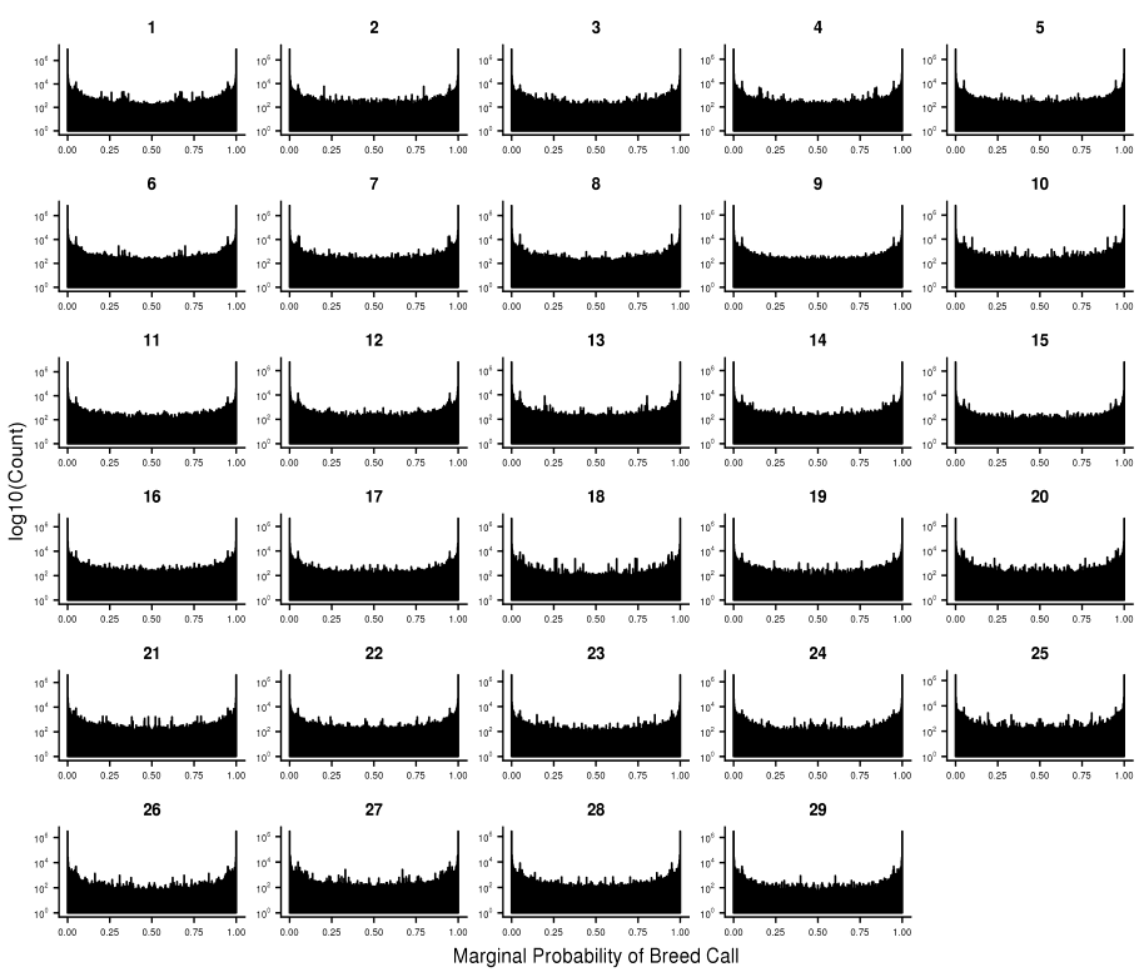

Figure S3.4. Distribution of Marginal Probabilities of Foundation Breed Assignment for Brangus. Distribution of the probability of reference population assignment for each CRF window. For example, for a given Brangus individual's haplotype for a CRF window, if RFmix estimated a 0.98 assignment to Angus, that for Brahman would be 0.02 . 
a
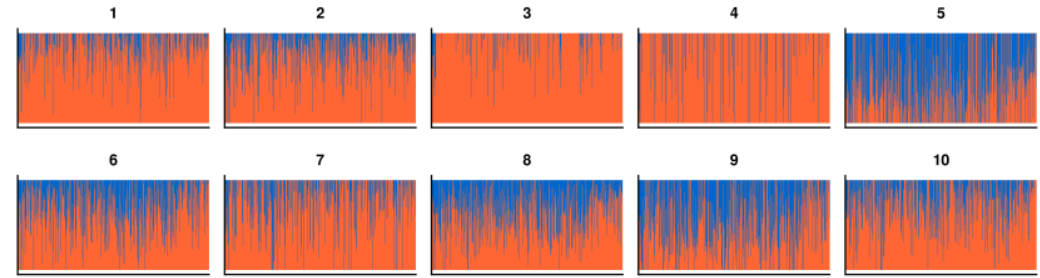

8

9

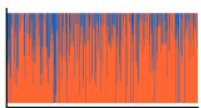

12

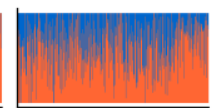

13

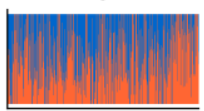

14
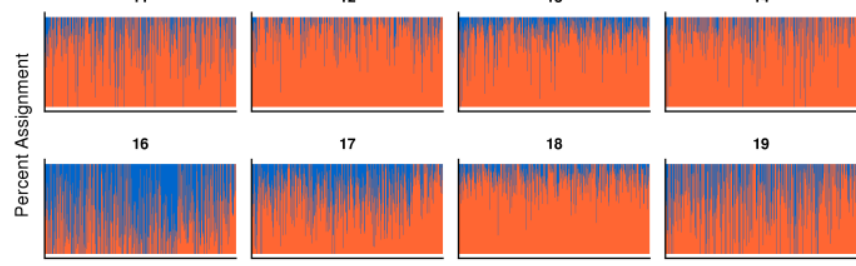

19

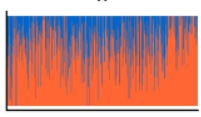

18
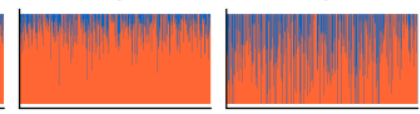

24

23

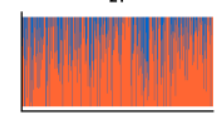

22

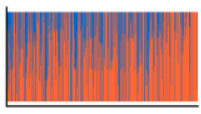

27

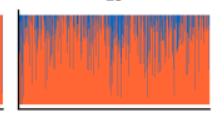

28

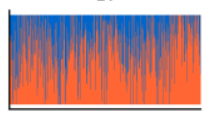

29
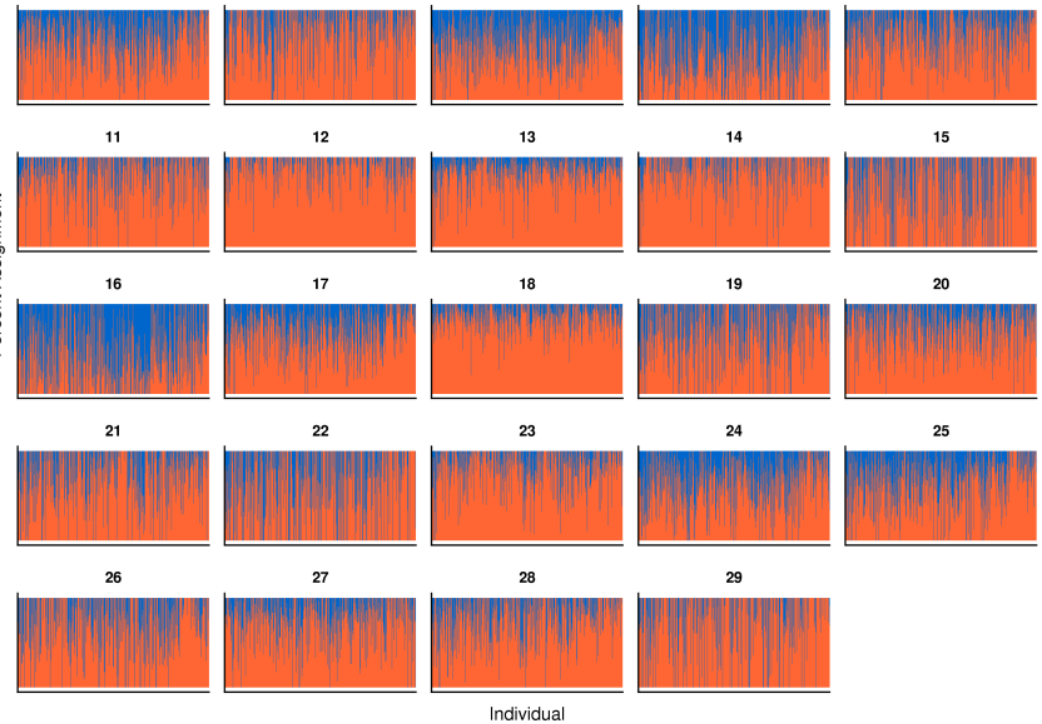

15

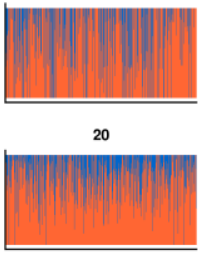

25

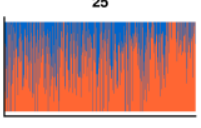

Brahman Shorthorn

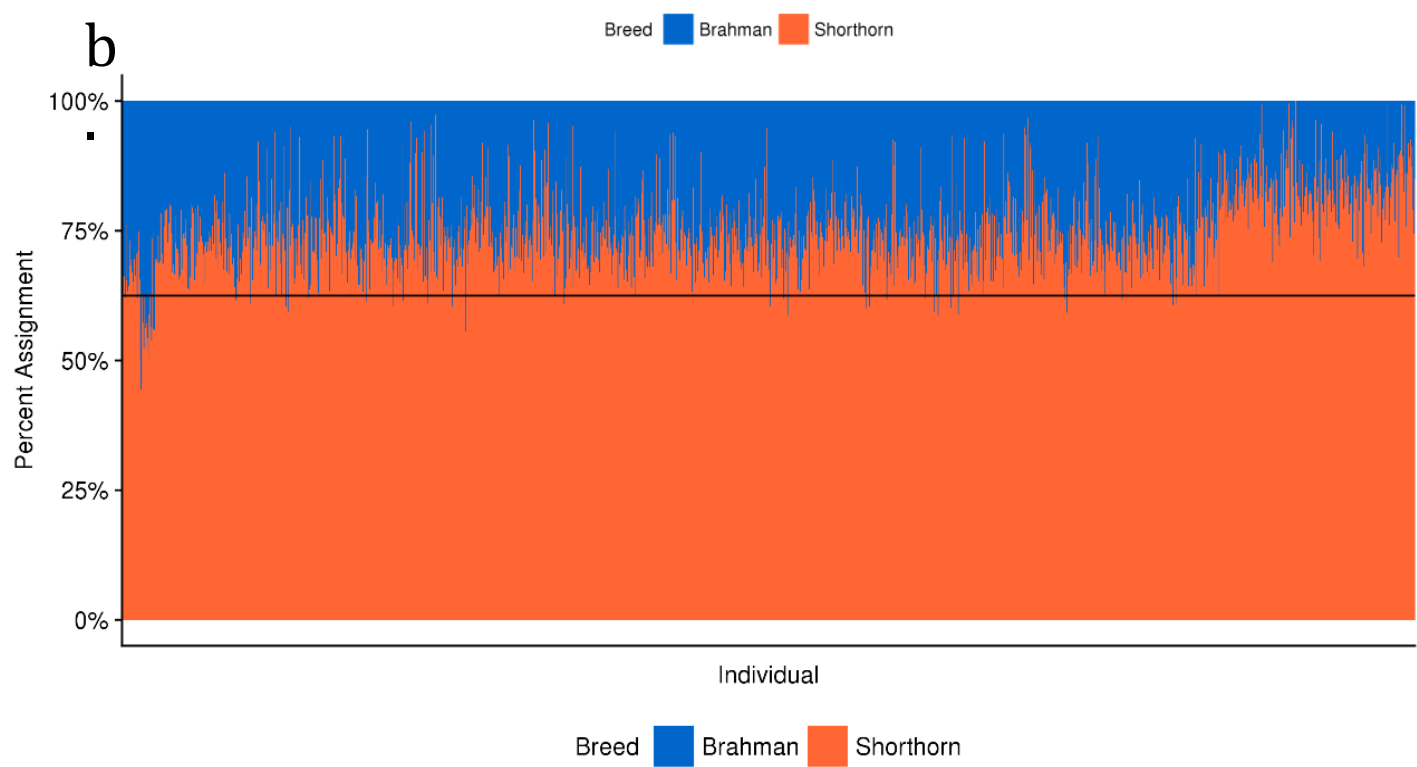

Figure S3.3. Santa Gertrudis Ancestry Estimation by Individual (Prior to Outlier Removal). (a.) Plot of RFmix global ancestry assignment for Santa Gertrudis individuals by chromosome. Individuals are represented by vertical bars. (b.) Plot of RFmix global ancestry assignment for Santa Gertrudis individuals genome-wide. Individuals are represented by vertical bars. The black line represents the proportion of Shorthorn (5/8) expected based on the creation of the Santa Gertrudis breed. 

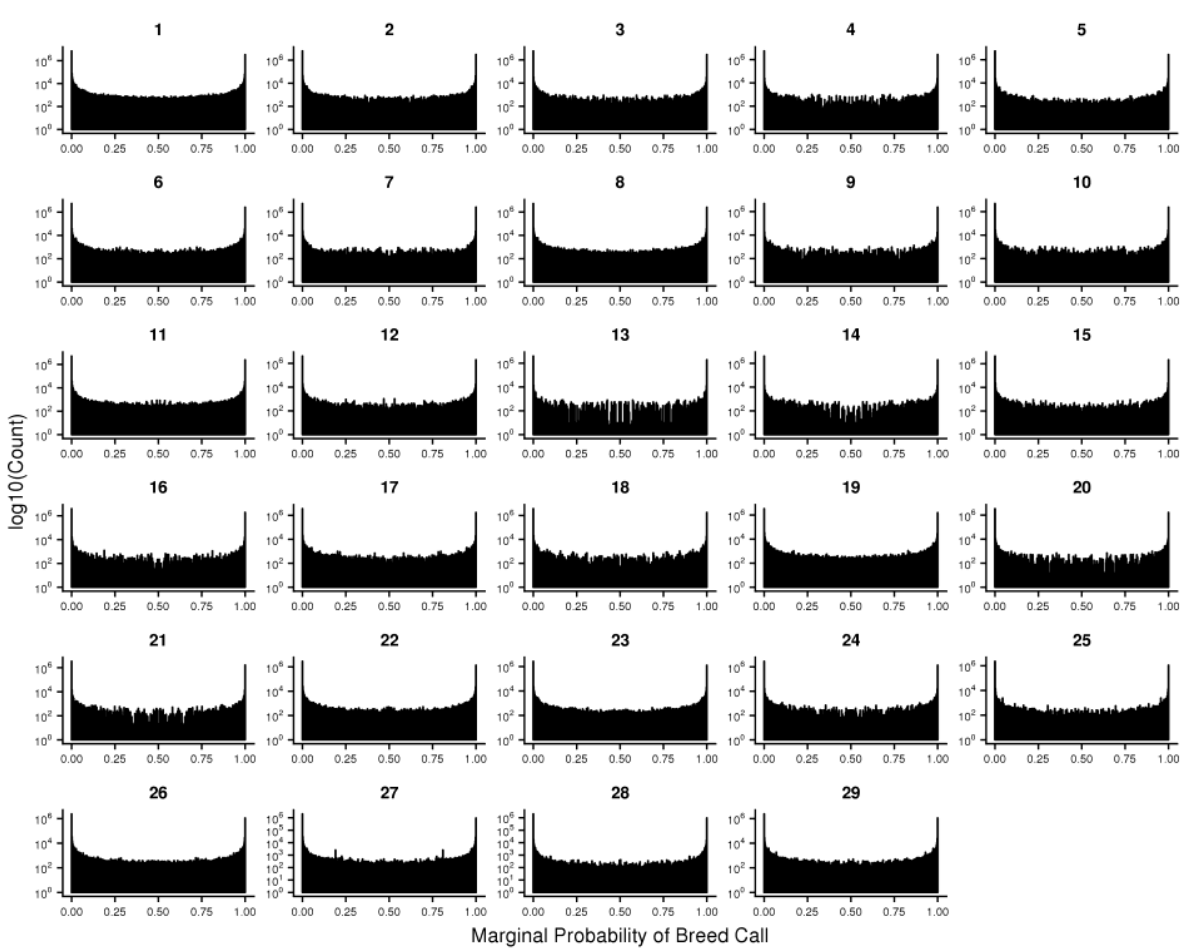

Figure S3.5. Distribution of Marginal Probabilities of Foundation Breed Assignment for Beefmaster. Distribution of the probability of reference population assignment for each CRF window. For example, for a given Beefmaster individual's haplotype for a CRF window, if RFmix estimated a 0.95 assignment to Brahman, 0.03 assignment to Hereford, that for Shorthorn would be 0.02 . 


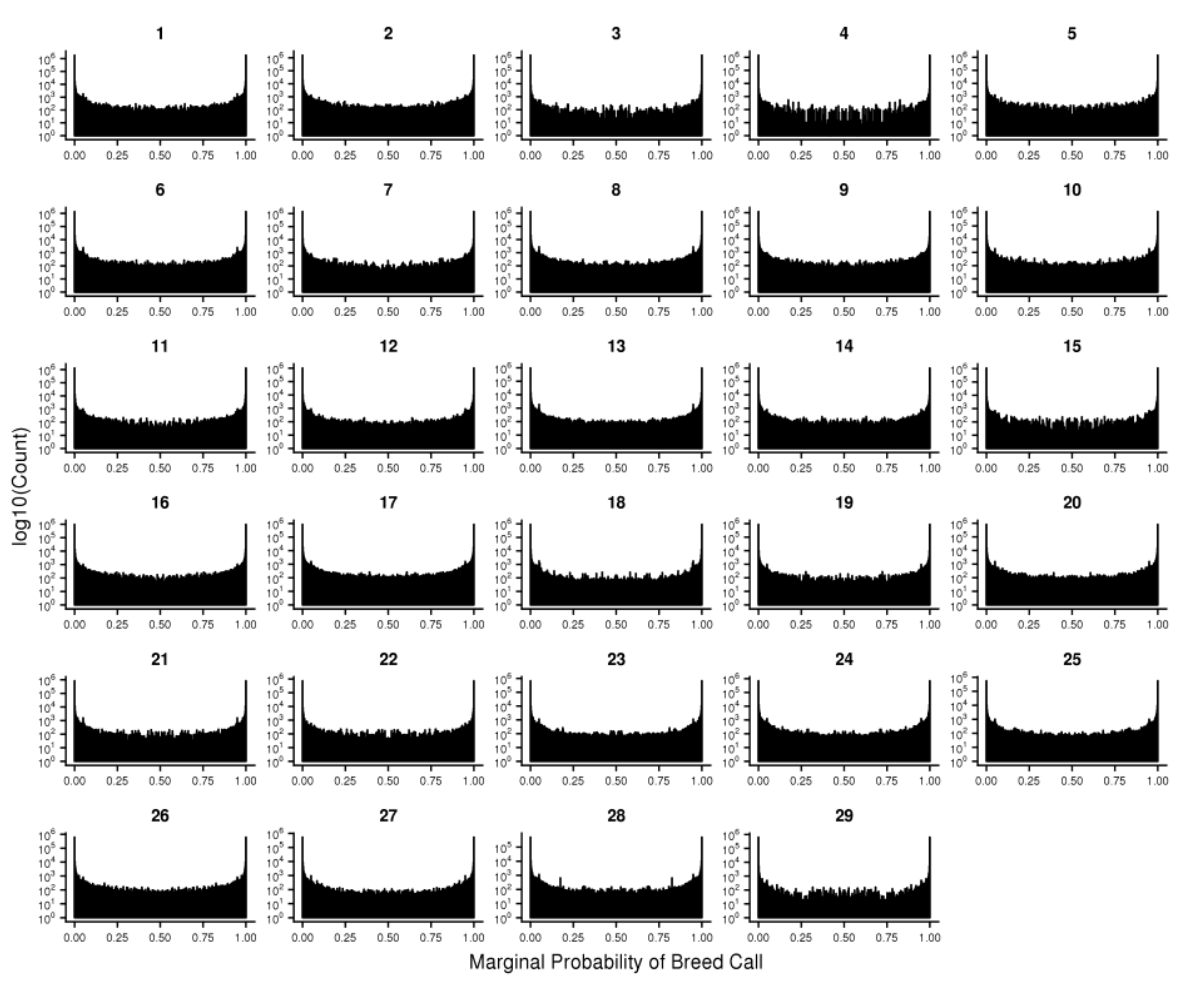

Figure S3.6. Distribution of Marginal Probabilities of Foundation Breed Assignment for Santa Gertrudis. Distribution of the probability of reference population assignment probability for each CRF window. For example, for a given Santa Gertrudis individual's haplotype for a CRF window, if RFmix estimated a 0.98 assignment to Shorthorn, that for Brahman would be 0.02 . 

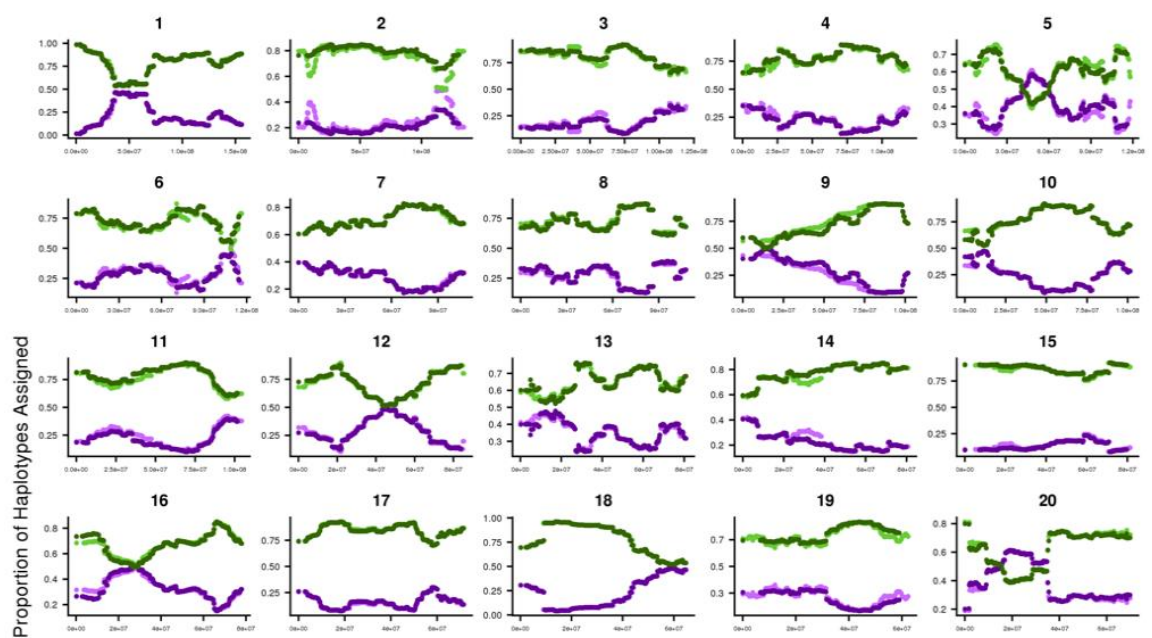

12

13
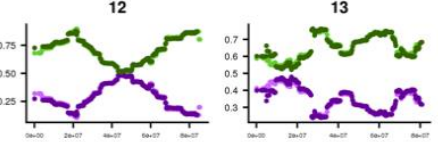

14
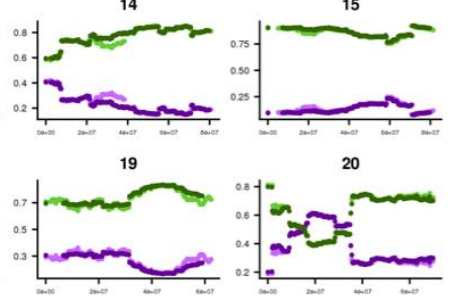

17

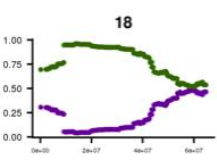

23

24

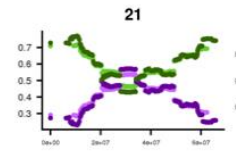

22
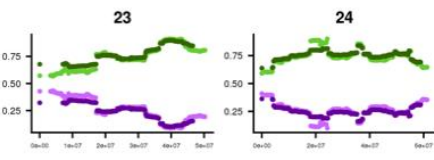

28
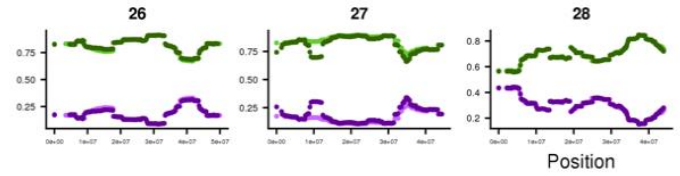

29

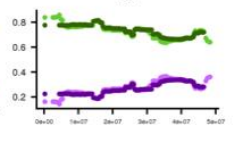

Breed:Reference Angus:Expanded Angus:Crumbler Brahman:Expanded Brahman:Crumbler

Figure S3.7. Brangus Reference Panel Comparison. Plot of RFmix most likely assigned ancestry based on reference panel. EXPANDED reference panel estimates are shown as Angus (red) and Brahman (black). CRUMBLER reference panel estimates are shown as Angus (blue) and Brahman (green). 

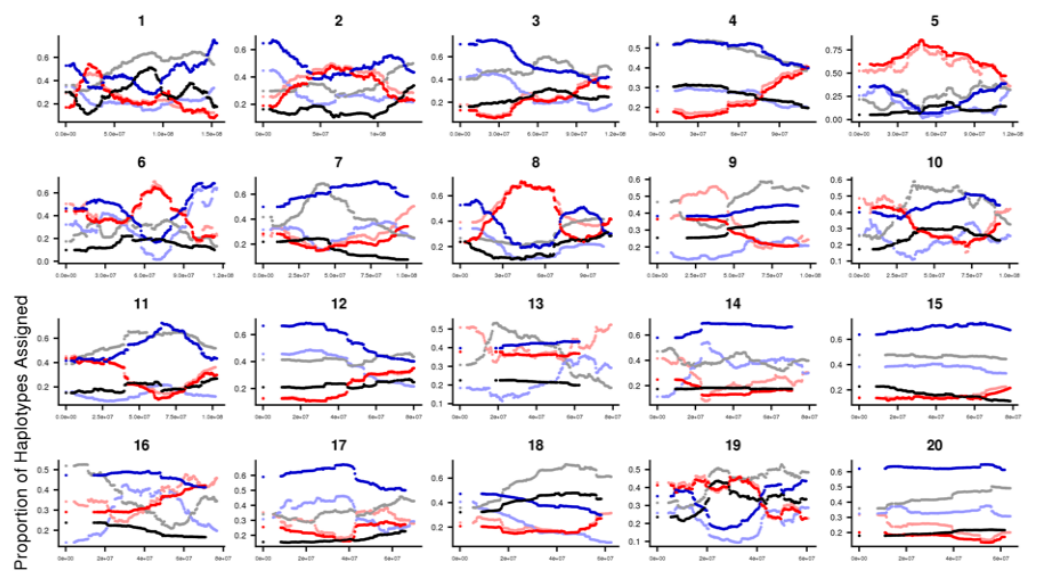

19
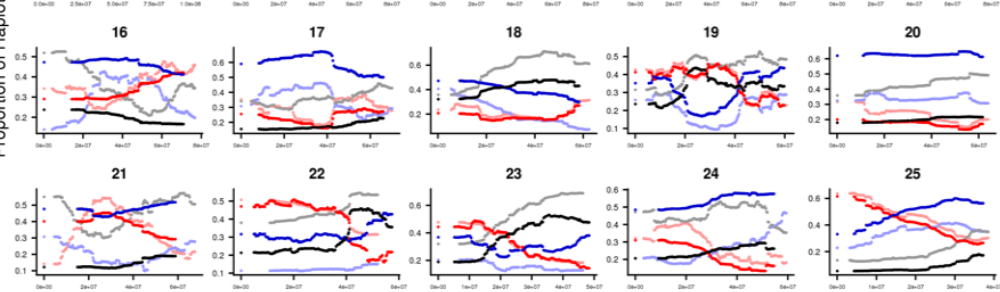

22
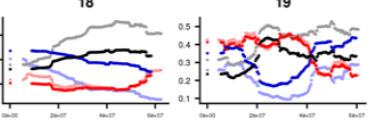

24

23
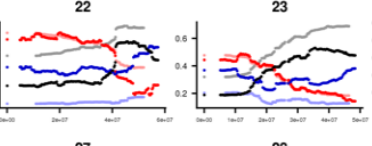

27
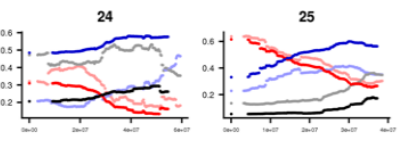

29

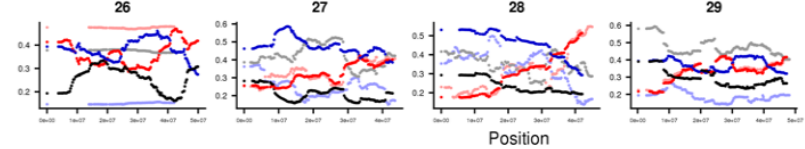

Breed:Reference Hereford:Expanded Shorthorn:Expanded Brahman:Expanded Hereford:Crumbler Shorthorn:Crumbler Brahman:Crumbler

Figure S3.8. Beefmaster Reference Panel Comparison. Plot of RFmix most likely assigned ancestry based on reference panel. EXPANDED reference panel estimates are shown as Hereford (red), Shorthorn (purple), and Brahman (black). CRUMBLER reference panel estimates are shown as Hereford (pink), Shorthorn (lavender), and Brahman (gray). 

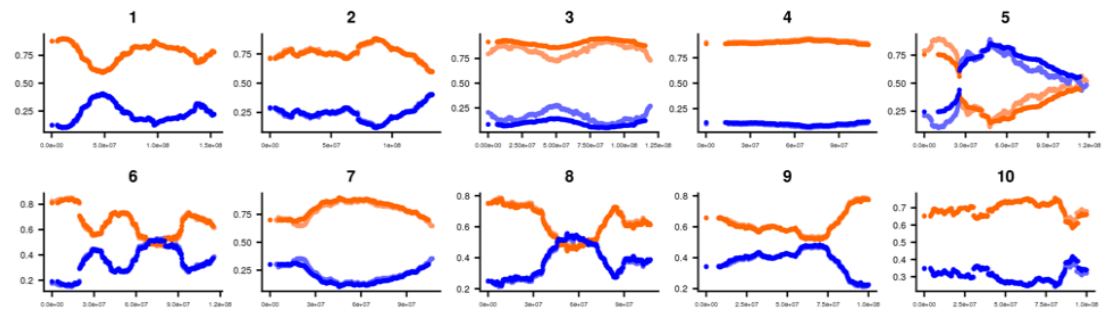

11

12

13
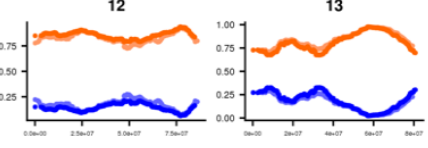

14

15

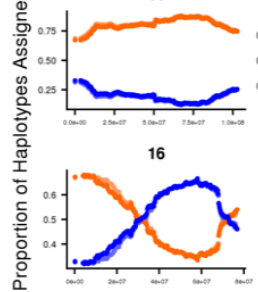

17
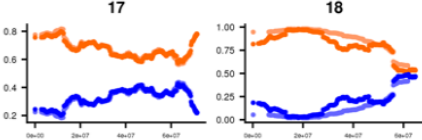

23
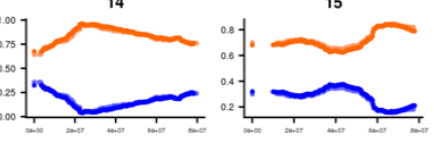

20
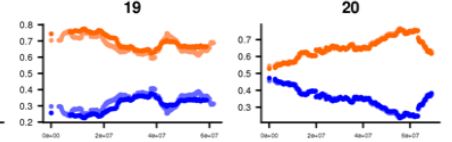

24

25
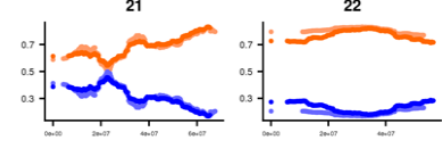

27
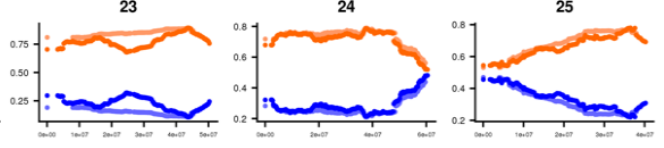

29
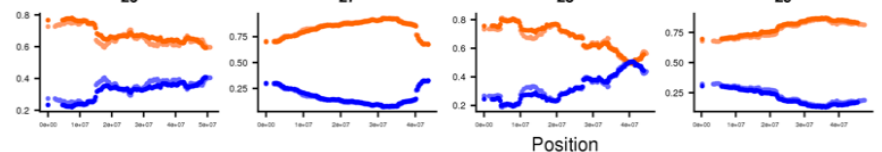

Breed:Reference Shorthorn:Expanded Shorthorn:Crumbler Brahman:Expanded Brahman:Crumble

Figure S3.9. Santa Gertrudis Reference Panel Comparison. Plot of RFmix most likely assigned ancestry based on reference panel. EXPANDED reference panel estimates are shown as Shorthorn (red) and Brahman (black). CRUMBLER reference panel estimates are shown as Shorthorn (pink) and Brahman (gray). 
Chapter 3 Supplementary Tables

Table S3.1. Most significant diverged regions from Brahman expectation for each American breeda.

\begin{tabular}{|c|c|c|c|c|c|c|c|c|}
\hline Chr. & $\begin{array}{l}\text { Start } \\
\text { (cM) }\end{array}$ & $\begin{array}{l}\text { End } \\
\text { (cM) }\end{array}$ & $\begin{array}{c}\text { \# of } \\
\text { SNPs }\end{array}$ & $\%$ Brahman & T-Statistic $^{b}$ & P-value & $-\log 10(p)$ & Benjamini-Hochberg Adj. P-value \\
\hline \multicolumn{9}{|c|}{ Brangus } \\
\hline 5 & 47.21 & 47.49 & 100 & 58.34 & -3.0146 & 0.0013 & 2.8906 & 0.2265 \\
\hline 5 & 47.49 & 47.74 & 100 & 58.42 & -3.0217 & 0.0013 & 2.9007 & 0.2265 \\
\hline 5 & 47.74 & 48.15 & 100 & 58.64 & -3.0423 & 0.0012 & 2.9304 & 0.2265 \\
\hline 5 & 48.15 & 48.59 & 100 & 58.55 & -3.0338 & 0.0012 & 2.9181 & 0.2265 \\
\hline 5 & 48.59 & 48.93 & 100 & 58.39 & -3.0192 & 0.0013 & 2.8971 & 0.2265 \\
\hline 20 & 18.78 & 19.10 & 100 & 61.20 & -3.2786 & 0.0005 & 3.2826 & 0.2265 \\
\hline 20 & 19.10 & 19.44 & 100 & 61.32 & -3.2897 & 0.0005 & 3.2997 & 0.2265 \\
\hline 20 & 19.44 & 19.74 & 100 & 61.29 & -3.2871 & 0.0005 & 3.2958 & 0.2265 \\
\hline 20 & 20.02 & 20.38 & 100 & 61.15 & -3.2740 & 0.0005 & 3.2756 & 0.2265 \\
\hline 20 & 20.72 & 21.01 & 100 & 61.12 & -3.2715 & 0.0005 & 3.2718 & 0.2265 \\
\hline 21 & 30.20 & 30.39 & 100 & 57.07 & -2.8977 & 0.0019 & 2.7260 & 0.2265 \\
\hline 21 & 30.39 & 30.66 & 100 & 57.08 & -2.8988 & 0.0019 & 2.7274 & 0.2265 \\
\hline 21 & 31.21 & 31.61 & 100 & 57.09 & -2.8993 & 0.0019 & 2.7281 & 0.2265 \\
\hline 21 & 31.61 & 32.06 & 100 & 57.09 & -2.8993 & 0.0019 & 2.7281 & 0.2265 \\
\hline 21 & 32.06 & 32.40 & 100 & 57.12 & -2.9018 & 0.0019 & 2.7316 & 0.2265 \\
\hline \multicolumn{9}{|c|}{ Beefmaster } \\
\hline 5 & 47.49 & 47.71 & 100 & 85.23 & -3.7039 & 0.0001 & 3.9741 & 0.0731 \\
\hline 5 & 47.71 & 48.13 & 100 & 85.23 & -3.7039 & 0.0001 & 3.9741 & 0.0731 \\
\hline 5 & 48.13 & 48.57 & 100 & 85.62 & -3.7302 & 0.0001 & 4.0192 & 0.0731 \\
\hline 5 & 48.57 & 48.92 & 100 & 85.62 & -3.7302 & 0.0001 & 4.0192 & 0.0731 \\
\hline 5 & 48.92 & 49.30 & 100 & 85.50 & -3.7220 & 0.0001 & 4.0052 & 0.0731 \\
\hline 5 & 49.30 & 49.52 & 100 & 85.49 & -3.7211 & 0.0001 & 4.0037 & 0.0731 \\
\hline
\end{tabular}




\begin{tabular}{|c|c|c|c|c|c|c|c|c|}
\hline 6 & 66.95 & 67.25 & 100 & 64.65 & -2.3020 & 0.0107 & 1.9719 & 0.2434 \\
\hline 6 & 67.25 & 67.55 & 100 & 64.75 & -2.3092 & 0.0105 & 1.9802 & 0.2404 \\
\hline 6 & 67.55 & 67.81 & 100 & 64.85 & -2.3156 & 0.0103 & 1.9875 & 0.2388 \\
\hline 6 & 67.81 & 68.01 & 100 & 65.01 & -2.3264 & 0.0100 & 2.0001 & 0.2352 \\
\hline 6 & 68.01 & 68.31 & 100 & 65.10 & -2.3328 & 0.0098 & 2.0074 & 0.2345 \\
\hline 8 & 42.96 & 43.29 & 100 & 71.12 & -2.7428 & 0.0030 & 2.5162 & 0.1873 \\
\hline 8 & 43.29 & 43.60 & 100 & 71.20 & -2.7482 & 0.0030 & 2.5234 & 0.1873 \\
\hline 8 & 43.60 & 43.85 & 100 & 70.36 & -2.6912 & 0.0036 & 2.4485 & 0.1874 \\
\hline 8 & 45.40 & 45.65 & 100 & 70.67 & -2.7120 & 0.0033 & 2.4757 & 0.1873 \\
\hline 8 & 45.65 & 45.96 & 100 & 70.45 & -2.6975 & 0.0035 & 2.4568 & 0.1874 \\
\hline 25 & 0.04 & 4.31 & 1850 & 61.51 & -2.0884 & 0.0184 & 1.7356 & 0.3467 \\
\hline 25 & 4.31 & 4.61 & 100 & 61.32 & -2.0757 & 0.0190 & 1.7222 & 0.3539 \\
\hline 25 & 4.61 & 4.86 & 100 & 60.99 & -2.0531 & 0.0200 & 1.6983 & 0.3669 \\
\hline & & & & & Sar & Gertrudi & & \\
\hline 5 & 47.71 & 48.13 & 100 & 84.94 & -3.9165 & 0.0000 & 4.3476 & 0.0214 \\
\hline 5 & 48.13 & 48.57 & 100 & 85.45 & -3.9518 & 0.0000 & 4.4114 & 0.0214 \\
\hline 5 & 48.57 & 48.92 & 100 & 84.99 & -3.9200 & 0.0000 & 4.3539 & 0.0214 \\
\hline 5 & 48.92 & 49.30 & 100 & 84.84 & -3.9095 & 0.0000 & 4.3349 & 0.0214 \\
\hline 5 & 49.30 & 49.52 & 100 & 84.63 & -3.8953 & 0.0000 & 4.3095 & 0.0214 \\
\hline 16 & 56.30 & 56.56 & 100 & 65.24 & -2.5661 & 0.0051 & 2.2889 & 0.2025 \\
\hline 16 & 57.09 & 57.46 & 100 & 65.29 & -2.5696 & 0.0051 & 2.2933 & 0.2025 \\
\hline 16 & 57.46 & 57.84 & 100 & 65.83 & -2.6067 & 0.0046 & 2.3400 & 0.1882 \\
\hline 16 & 57.84 & 58.08 & 100 & 65.94 & -2.6138 & 0.0045 & 2.3490 & 0.1866 \\
\hline 16 & 58.08 & 58.36 & 100 & 66.58 & -2.6579 & 0.0039 & 2.4055 & 0.1686 \\
\hline
\end{tabular}

${ }^{a}$ Regions were extracted based on a Benjamini-Hochberg adjusted $p$-value of $\leq 0.4$, were grouped by chromosome and the 5 most significant regions for each chromosome were retained for downstream analysis for each breed.

${ }^{\mathrm{b}}$ The t-statistic for each window was computed for each breed by: (-abs((Brahman Proportion-Genome Wide Brahman Proportion)/Genome Wide Standard Deviation)). Genome wide proportions are reported in Table 3.4. 


\section{Chapter 4 Supplementary Tables}

Table S4.1. PVE, log likelihood at MLE and effect estimates for Model 1 by trait.

\begin{tabular}{|c|c|c|c|c|c|}
\hline & ADG & DMI & MMWT & \multicolumn{2}{|c|}{ RFI } \\
\hline $\begin{array}{l}\text { Log- } \\
\text { Likelihood }\end{array}$ & -9194.23 & -26530.8 & -43026.2 & -229 & 42.6 \\
\hline PVE & $0.2174 \quad(0.0141)$ & $0.2791 \quad(0.0145)$ & $0.3271 \quad(0.0150)$ & 0.2392 & $(0.0150)$ \\
\hline Beta $_{\text {int }}{ }^{b}$ & $-0.0012 \quad(0.0047)$ & $-0.0101 \quad(0.0212)$ & $-0.0572 \quad(0.0883)$ & -0.0017 & $(0.0157)$ \\
\hline Beta $_{A D G}{ }^{b}$ & & & & 1.5665 & $(0.0345)$ \\
\hline Beta $_{\text {ммшт }}{ }^{b}$ & & & & 0.1142 & $(0.0017)$ \\
\hline
\end{tabular}

a. RFI was estimated using DMI as the dependent variable and ADG and MMWT fit as partial regressions in the model.

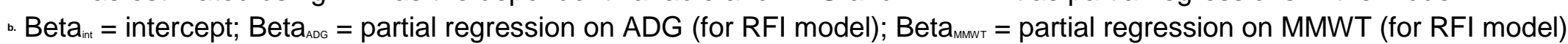


Table S4.2. PVE, log likelihood at MLE, and effect estimates for Model 2 by trait.

\begin{tabular}{|c|c|c|c|c|c|c|c|c|}
\hline & \multicolumn{2}{|c|}{ ADG } & \multicolumn{2}{|c|}{ DMI } & \multicolumn{2}{|c|}{ MMWT } & \multicolumn{2}{|c|}{$\left.\mathbf{R F}\right|^{\mathbf{a}}$} \\
\hline Log- & \multicolumn{2}{|c|}{-9185.43} & \multicolumn{2}{|c|}{-26520.2} & \multicolumn{2}{|c|}{-43018.7} & \multicolumn{2}{|c|}{-22934.3} \\
\hline PVE & 0.2114 & $(0.0144)$ & 0.2731 & $(0.0148)$ & 0.3233 & $(0.0152)$ & 0.2374 & $(0.0151)$ \\
\hline Beta $_{\text {int }}{ }^{b}$ & -0.2008 & $(0.0732)$ & -1.0210 & $(0.3517)$ & -3.1821 & (1.5253) & -0.5425 & $(0.2519)$ \\
\hline Beta $_{A N}{ }^{b}$ & 0.2180 & $(0.1100)$ & 1.6492 & $(0.5367)$ & 5.4463 & (2.3567) & 0.8399 & (0.3813) \\
\hline Beta $_{\mathrm{CH}}{ }^{\mathrm{b}}$ & 0.2236 & $(0.1064)$ & 0.8504 & $(0.5254)$ & 5.2897 & (2.3261) & 0.3917 & (0.3708) \\
\hline Beta $_{H F}{ }^{b}$ & 0.1892 & $(0.1411)$ & 0.2726 & $(0.6863)$ & -2.6102 & (2.9840) & 0.5913 & (0.4897) \\
\hline Beta $_{\mathrm{GL}}{ }^{\mathrm{b}}$ & 0.2683 & $(0.1091)$ & 0.5783 & $(0.5387)$ & 2.3991 & (2.3788) & 0.0265 & (0.3806) \\
\hline Beta $_{\llcorner M}$ & -0.0988 & $(0.1329)$ & -0.3429 & $(0.6598)$ & 0.3089 & (2.9271) & 0.1681 & (0.4636) \\
\hline Beta $_{\mathrm{RA}}{ }^{\mathrm{b}}$ & 0.2324 & $(0.1268)$ & 2.3067 & $(0.6233)$ & 5.9746 & (2.7429) & 1.2598 & (0.4409) \\
\hline Betasm $^{b}$ & 0.3316 & $(0.1295)$ & -0.1543 & $(0.6363)$ & 4.1389 & $(2.8101)$ & -0.7225 & (0.4498) \\
\hline Beta $_{A D G^{b}}$ & & & & & & & 1.5658 & $(0.0345)$ \\
\hline Beta $_{\text {ммшт }}{ }^{b}$ & & & & & & & 0.1141 & $(0.0017)$ \\
\hline
\end{tabular}


Table S4.3. PVE, log likelihood at MLE, and effect estimates for Model 3 by trait.

\begin{tabular}{|c|c|c|c|c|c|c|c|c|}
\hline & \multicolumn{2}{|c|}{ ADG } & \multicolumn{2}{|c|}{ DMI } & \multicolumn{2}{|c|}{ MMWT } & \multicolumn{2}{|c|}{ RFI } \\
\hline $\begin{array}{c}\text { Log } \\
\text { Likelihood }\end{array}$ & \multicolumn{2}{|c|}{-9163.54} & \multicolumn{2}{|c|}{-26498.2} & \multicolumn{2}{|c|}{-42971} & \multicolumn{2}{|c|}{-22918.1} \\
\hline PVE & 0.2103 & $(0.0146)$ & 0.2722 & $(0.0150)$ & 0.3281 & $(0.0153)$ & 0.2387 & (0.0152) \\
\hline Beta $_{i n t}{ }^{b}$ & -0.2258 & $(0.0758)$ & -0.8599 & $(0.3649)$ & -1.1018 & $(1.5860)$ & -0.5295 & $(0.2611)$ \\
\hline Beta $_{A N}{ }^{b}$ & 0.3244 & $(0.1245)$ & 1.0554 & $(0.6076)$ & 1.7710 & (2.6787) & 0.5783 & (0.4319) \\
\hline Beta $_{\mathrm{CH}}{ }^{\mathrm{b}}$ & 0.4491 & $(0.1617)$ & 1.8757 & $(0.8019)$ & 0.5072 & $(3.5756)$ & 1.5181 & $(0.5662)$ \\
\hline Beta $_{H F}{ }^{b}$ & 0.1953 & $(0.1420)$ & 0.1807 & $(0.6906)$ & -3.6053 & $(3.0006)$ & 0.5764 & $(0.4936)$ \\
\hline Beta $_{\mathrm{GL}} \mathrm{b}^{\mathrm{b}}$ & 0.1872 & $(0.1465)$ & -0.1866 & $(0.7318)$ & -0.5301 & (3.2704) & -0.3916 & $(0.5146)$ \\
\hline BetaLm & 0.1266 & $(0.1566)$ & 0.4978 & $(0.7767)$ & -2.1979 & (3.4663) & 0.2694 & (0.5471) \\
\hline Beta $_{\mathrm{RA}}{ }^{\mathrm{b}}$ & 0.4587 & $(0.1539)$ & 1.6237 & $(0.7666)$ & -1.3682 & $(3.4116)$ & 0.8707 & (0.5395) \\
\hline Betasm $^{b}$ & 0.3095 & $(0.1902)$ & -0.5117 & $(0.9202)$ & -4.0038 & (4.0199) & -0.2463 & (0.6568) \\
\hline Beta $_{A N^{*} C H^{b}}$ & -0.4076 & $(0.2976)$ & -1.6214 & $(1.4191)$ & 9.7835 & $(6.1530)$ & -2.2508 & (1.0235) \\
\hline Beta $_{A N^{*} H F^{b}}$ & 0.1412 & $(0.2623)$ & 0.6622 & (1.2435) & 1.4558 & $(5.3180)$ & -0.1318 & (0.9003) \\
\hline Beta $_{A N^{*} G L}{ }^{b}$ & 0.5565 & $(0.2479)$ & 4.3489 & (1.1733) & 9.1507 & $(5.0480)$ & 3.0562 & $(0.8474)$ \\
\hline Beta $_{A N^{*} L M^{b}}$ & -0.5153 & $(0.4078)$ & -0.2570 & $(1.9060)$ & 10.5269 & $(8.2356)$ & 0.0897 & (1.3809) \\
\hline Beta $_{A N^{*} R A^{b}}$ & -0.8898 & $(0.2696)$ & 0.9663 & (1.3072) & -0.1612 & $(5.6583)$ & 2.0727 & $(0.9360)$ \\
\hline Beta $_{A N^{*} S M^{b}}$ & 0.0113 & $(0.2912)$ & 2.5177 & $(1.3871)$ & 20.5715 & $(5.9004)$ & -0.4219 & (1.0032) \\
\hline Beta $_{\mathrm{CH}^{*} \mathrm{HF}^{\mathrm{b}}}$ & -0.3844 & $(0.3824)$ & -1.3335 & $(1.8291)$ & 3.7443 & (7.9091) & -1.4114 & (1.3168) \\
\hline Beta $_{\mathrm{CH}^{*} \mathrm{GL}}{ }^{\mathrm{b}}$ & -1.3902 & $(0.6952)$ & -1.2914 & (3.2772) & 1.9090 & (13.8795) & -0.6235 & (2.3778) \\
\hline Beta $_{C H^{*}\left\llcorner M^{b}\right.}$ & -1.3696 & $(0.7020)$ & -4.0324 & (3.3327) & -1.6276 & (14.1303) & -0.5313 & (2.4135) \\
\hline Beta $_{C H^{\star} R A^{b}}$ & 0.6365 & $(0.5901)$ & -1.2591 & $(2.7934)$ & 29.9419 & (12.0007) & -3.0104 & (2.0261) \\
\hline Beta $_{\mathrm{CH}^{*} \mathrm{Sm}^{\mathrm{b}}}$ & -0.7992 & $(0.4465)$ & -3.4012 & (2.0972) & -1.9983 & $(9.0637)$ & -2.8748 & (1.5185) \\
\hline Beta $_{\mathrm{HF}^{\star} \mathrm{GL}^{\mathrm{b}}}^{\mathrm{b}}$ & -0.4007 & $(0.3772)$ & -0.2330 & $(1.7977)$ & -1.5245 & $(7.8678)$ & -0.4681 & (1.2931) \\
\hline Beta $_{H^{*} F^{*} M^{b}}$ & -1.2945 & $(0.6239)$ & -2.1741 & (2.9438) & 0.1561 & (12.5658) & 0.8083 & (2.1350) \\
\hline Beta $_{H F^{\star} R A^{b}}$ & -0.3616 & $(0.2825)$ & 4.0444 & (1.3585) & 37.6126 & $(5.8584)$ & 0.7960 & $(0.9784)$ \\
\hline
\end{tabular}




\begin{tabular}{|c|c|c|c|c|c|c|c|c|}
\hline Beta $_{H^{*} \mathrm{Sm}^{\mathrm{b}}}$ & 0.0663 & $(0.5481)$ & -1.0842 & (2.6039) & -16.7251 & (11.0702) & 1.4245 & $(1.8903)$ \\
\hline Beta $_{\mathrm{GL}^{\star} \mathrm{LM}^{\mathrm{b}}}$ & 1.0191 & $(0.8500)$ & -4.4622 & $(4.0402)$ & -0.2297 & (17.2463) & -2.8740 & (2.9325) \\
\hline Beta $_{G^{*}{ }^{*} R A^{b}}$ & -0.0246 & $(0.4986)$ & 0.1422 & (2.3331) & -1.2109 & (10.0156) & 0.1678 & (1.6905) \\
\hline Beta $_{\mathrm{GL}^{\star} \mathrm{SM}}{ }^{\mathrm{b}}$ & 0.2033 & $(0.5413)$ & -1.3708 & $(2.5655)$ & 10.9767 & (11.0018) & -1.7751 & (1.8549) \\
\hline Beta $_{L M^{\star} R A^{b}}$ & -1.2448 & $(0.8401)$ & -3.5714 & (3.9157) & 0.4614 & (16.9484) & 0.4414 & (2.8424) \\
\hline Beta $_{L M}{ }^{*} M^{b}$ & -0.2087 & $(0.6911)$ & -4.8139 & (3.2447) & 7.2348 & $(13.9319)$ & -1.3736 & (2.3538) \\
\hline Beta $_{\mathrm{RA}^{*} \mathrm{SM}^{\mathrm{b}}}$ & 0.3133 & $(0.4646)$ & 1.2467 & $(2.2130)$ & 24.0680 & $(9.4244)$ & -1.2998 & (1.5986) \\
\hline Beta $_{A D G}{ }^{b}$ & & & & & & & 1.5639 & $(0.0345)$ \\
\hline Beta $_{м м ш т}{ }^{b}$ & & & & & & & 0.1142 & $(0.0017)$ \\
\hline
\end{tabular}

a.RFI was estimated using DMI as the dependent variable and ADG and MMWT fit as partial regressions in the model.

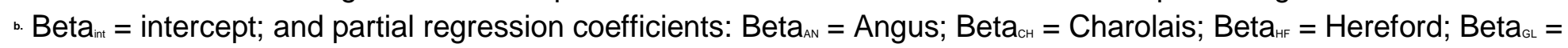

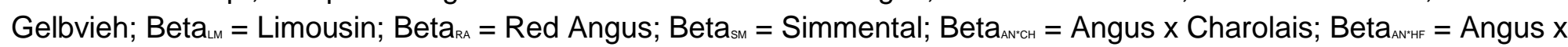

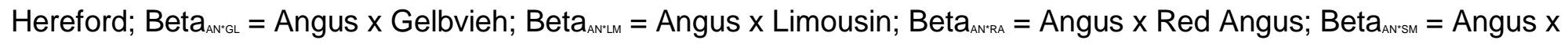

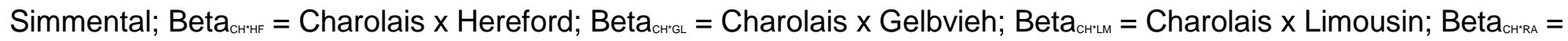

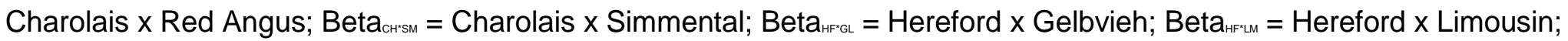

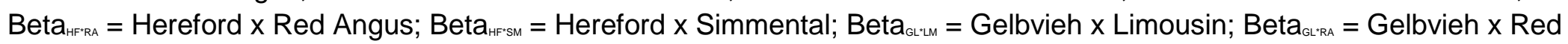

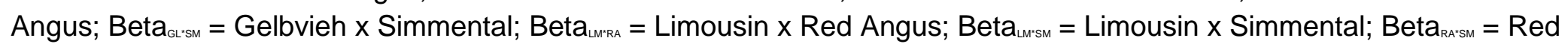
Angus $x$ Simmental; Beta $_{\text {Аос }}=$ ADG (for RFI model); Beta $_{\text {митт }}=$ MMWT (for RFI model) 
Table S4.4. Genomic inflation factors $(\lambda)$ associated with $Q-Q$ plot $p$-values.

\begin{tabular}{cccc}
\hline & Model 1 & Model 2 & Model 3 \\
\hline ADG & 1.190 & 1.189 & 1.188 \\
DMI & 1.227 & 1.228 & 1.226 \\
MMWT & 1.312 & 1.312 & 1.301 \\
RFI & 1.228 & 1.229 & 1.229 \\
\hline
\end{tabular}




\section{REFERENCES}

1. Liu Y, Nyunoya T, Leng S, Belinsky SA, Tesfaigzi Y, Bruse S. Softwares and methods for estimating genetic ancestry in human populations. Hum Genomics. 2013;7:1.

2. Tian C, Gregersen PK, Seldin MF. Accounting for ancestry: population substructure and genome-wide association studies. Hum Mol Genet. 2008;17:R143-50.

3. Pasaniuc B, Zaitlen N, Lettre G, Chen GK, Tandon A, Kao WHL, et al. Enhanced statistical tests for GWAS in admixed populations: assessment using African Americans from CARe and a Breast Cancer Consortium. PLoS Genet. 2011;7:e1001371.

4. Pritchard JK, Stephens M, Donnelly P. Inference of population structure using multilocus genotype data. Genetics. 2000;155:945-59.

5. Tishkoff SA, Kidd KK. Implications of biogeography of human populations for 'race' and medicine. Nat Genet. 2004.

https://www.nature.com/ng/journal/v36/n11s/full/ng1438.html.

6. Burnett MS, Strain KJ, Lesnick TG, de Andrade M, Rocca WA, Maraganore DM. Reliability of self-reported ancestry among siblings: implications for genetic association studies. Am J Epidemiol. 2006;163:486-92.

7. Helgason A, Yngvadóttir B, Hrafnkelsson B, Gulcher J, Stefánsson K. An Icelandic example of the impact of population structure on association studies. Nat Genet. 2005;37:90-5.

8. Campbell CD, Ogburn EL, Lunetta KL, Lyon HN, Freedman ML, Groop LC, et al. Demonstrating stratification in a European American population. Nat Genet. 2005;37:868.

9. Tian C, Hinds DA, Shigeta R, Kittles R, Ballinger DG, Seldin MF. A Genomewide Single-Nucleotide-Polymorphism Panel with High Ancestry Information for African American Admixture Mapping. Am J Hum Genet. 2006;79:640-9.

10. Price AL, Butler J, Patterson N, Capelli C, Pascali VL, Scarnicci F, et al. Discerning the ancestry of European Americans in genetic association studies. PLoS Genet. 2008;4:e236.

11. Bersaglieri T, Sabeti PC, Patterson N, Vanderploeg T, Schaffner SF, Drake $\mathrm{JA}$, et al. Genetic signatures of strong recent positive selection at the lactase gene. Am J Hum Genet. 2004;74:1111-20. 
12. Enattah NS, Sahi T, Savilahti E, Terwilliger JD, Peltonen L, Järvelä I. Identification of a variant associated with adult-type hypolactasia. Nat Genet. 2002;30:233-7.

13. Gonzalez Burchard E, Borrell LN, Choudhry S, Naqvi M, Tsai H-J, RodriguezSantana JR, et al. Latino populations: a unique opportunity for the study of race, genetics, and social environment in epidemiological research. Am J Public Health. 2005;95:2161-8.

14. Shriner D. Overview of admixture mapping. Curr Protoc Hum Genet. 2013;Chapter 1:Unit 1.23.

15. Brehm JM, Celedón JC. Chronic obstructive pulmonary disease in Hispanics. Am J Respir Crit Care Med. 2008;177:473-8.

16. Freedman ML, Haiman CA, Patterson N, McDonald GJ, Tandon A, Waliszewska A, et al. Admixture mapping identifies 8q24 as a prostate cancer risk locus in African-American men. Proc Natl Acad Sci U S A. 2006;103:1406873.

17. Smith MW, Patterson N, Lautenberger JA, Truelove AL, McDonald GJ, Waliszewska $A$, et al. A high-density admixture map for disease gene discovery in african americans. Am J Hum Genet. 2004;74:1001-13.

18. Kassahun Y, Mattiangeli V, Ameni G, Hailu E, Aseffa A, Young DB, et al. Admixture mapping of tuberculosis and pigmentation-related traits in an AfricanEuropean hybrid cattle population. Front Genet. 2015;6:210.

19. Hoggart CJ, Parra EJ, Shriver MD, Bonilla C, Kittles RA, Clayton DG, et al. Control of confounding of genetic associations in stratified populations. Am J Hum Genet. 2003;72:1492-504.

20. Kosoy R, Nassir R, Tian C, White PA, Butler LM, Silva G, et al. Ancestry informative marker sets for determining continental origin and admixture proportions in common populations in America. Hum Mutat. 2009;30:69-78.

21. Nassir R, Kosoy R, Tian C, White PA, Butler LM, Silva G, et al. An ancestry informative marker set for determining continental origin: validation and extension using human genome diversity panels. BMC Genet. 2009;10:39.

22. Seldin MF, Price AL. Application of ancestry informative markers to association studies in European Americans. PLoS Genet. 2008;4:e5.

23. Seldin MF, Pasaniuc B, Price AL. New approaches to disease mapping in admixed populations. Nat Rev Genet. 2011;12:523-8. 
24. Pino-Yanes M, Gignoux CR, Galanter JM, Levin AM, Campbell CD, Eng C, et al. Genome-wide association study and admixture mapping reveal new loci associated with total IgE levels in Latinos. J Allergy Clin Immunol. 2015;135:1502-10.

25. Tang H, Siegmund DO, Johnson NA, Romieu I, London SJ. Joint testing of genotype and ancestry association in admixed families. Genet Epidemiol. 2010;34:783-91.

26. Pritchard JK, Stephens M, Rosenberg NA, Donnelly P. Association mapping in structured populations. Am J Hum Genet. 2000;67:170-81.

27. Alexander DH, Novembre J, Lange K. Fast model-based estimation of ancestry in unrelated individuals. Genome Res. 2009;19:1655-64.

28. International HapMap Consortium, Frazer KA, Ballinger DG, Cox DR, Hinds DA, Stuve LL, et al. A second generation human haplotype map of over 3.1 million SNPs. Nature. 2007;449:851-61.

29. 1000 Genomes Project Consortium, Auton A, Brooks LD, Durbin RM, Garrison EP, Kang HM, et al. A global reference for human genetic variation. Nature. 2015;526:68-74.

30. Gelernter J, Kranzler HR, Sherva R, Almasy L, Koesterer R, Smith AH, et al. Genome-wide association study of alcohol dependence:significant findings in African- and European-Americans including novel risk loci. Mol Psychiatry. 2014;19:41-9.

31. Huang L, Li Y, Singleton AB, Hardy JA, Abecasis G, Rosenberg NA, et al. Genotype-imputation accuracy across worldwide human populations. Am J Hum Genet. 2009;84:235-50.

32. Delaneau O, Marchini J, McVean GA. Integrating sequence and array data to create an improved 1000 Genomes Project haplotype reference panel. Nature. 2014. https://www.nature.com/articles/ncomms4934.

33. Khor SS, Yang W, Kawashima M, Kamitsuji S. High-accuracy imputation for HLA class I and II genes based on high-resolution SNP data of populationspecific references. The. 2015. https://www.nature.com/articles/tpj20154.

34. Bryc K, Durand EY, Macpherson JM, Reich D. The genetic ancestry of african americans, latinos, and european Americans across the United States. of Human Genetics. 2015.

https://www.sciencedirect.com/science/article/pii/S0002929714004765.

35. Browning SR, Grinde K, Plantinga A. Local ancestry inference in a large us- 
based hispanic/latino study: Hispanic community health study/study of latinos (hchs/sol). G3: Genes, Genomes. 2016.

http://www.g3journal.org/content/early/2016/03/21/g3.116.028779.abstract.

36. Song Q, Xu W, Li W, He S, Liu J, Wang G, et al. Accurate haplotype imputation with individualized ancestry-adjusted reference panels. Genomics. 2017. doi:10.1016/j.ygeno.2017.11.005.

37. Decker JE, Pires JC, Conant GC, McKay SD, Heaton MP, Chen K, et al. Resolving the evolution of extant and extinct ruminants with high-throughput phylogenomics. Proc Natl Acad Sci U S A. 2009;106:18644-9.

38. Decker JE, McKay SD, Rolf MM, Kim J, Molina Alcalá A, Sonstegard TS, et al. Worldwide patterns of ancestry, divergence, and admixture in domesticated cattle. PLoS Genet. 2014;10:e1004254.

39. Rolf MM, Taylor JF, Schnabel RD, McKay SD, McClure MC, Northcutt SL, et al. Impact of reduced marker set estimation of genomic relationship matrices on genomic selection for feed efficiency in Angus cattle. BMC Genet. 2010;11:24.

40. VanRaden PM, Cooper TA. Genomic evaluations and breed composition for crossbred U.S. dairy cattle. Interbull Bulletin. 2015;0.

https://journal.interbull.org/index.php/ib/article/view/1370. Accessed 3 May 2018.

41. Lamy E, van Harten S, Sales-Baptista E, Guerra MMM, de Almeida AM. Factors Influencing Livestock Productivity. In: Sejian V, Naqvi SMK, Ezeji T, Lakritz J, Lal R, editors. Environmental Stress and Amelioration in Livestock Production. Berlin, Heidelberg: Springer Berlin Heidelberg; 2012. p. 19-51.

42. Gobena M, Elzo MA, Mateescu RG. Population Structure and Genomic Breed Composition in an Angus-Brahman Crossbred Cattle Population. Front Genet. 2018;9:90.

43. Meuwissen TH, Hayes BJ, Goddard ME. Prediction of total genetic value using genome-wide dense marker maps. Genetics. 2001;157:1819-29.

44. Moghaddar N, Swan AA, van der Werf JHJ. Comparing genomic prediction accuracy from purebred, crossbred and combined purebred and crossbred reference populations in sheep. Genet Sel Evol. 2014;46:58.

45. Taylor JF, Taylor KH, Decker JE. Holsteins are the genomic selection poster cows. Proc Natl Acad Sci U S A. 2016;113:7690-2.

46. VanRaden PM, Van Tassell CP, Wiggans GR, Sonstegard TS, Schnabel RD, Taylor JF, et al. Invited review: reliability of genomic predictions for North American Holstein bulls. J Dairy Sci. 2009;92:16-24. 
47. García-Ruiz A, Cole JB, VanRaden PM, Wiggans GR, Ruiz-López FJ, Van Tassell CP. Changes in genetic selection differentials and generation intervals in US Holstein dairy cattle as a result of genomic selection. Proc Natl Acad Sci U S A. 2016;113:E3995-4004.

48. Berry DP, Garcia JF, Garrick DJ. Development and implementation of genomic predictions in beef cattle. 2016. http://t-

stor.teagasc.ie/handle/11019/1015.

49. Pszczola M, Strabel T, Mulder HA, Calus MPL. Reliability of direct genomic values for animals with different relationships within and to the reference population. J Dairy Sci. 2012;95:389-400.

50. Saatchi M, Beever JE, Decker JE, Faulkner DB, Freetly HC, Hansen SL, et al. QTLs associated with dry matter intake, metabolic mid-test weight, growth and feed efficiency have little overlap across 4 beef cattle studies. BMC Genomics. 2014;15:1004.

51. Seabury CM, Oldeschulte DL, Saatchi M, Beever JE, Decker JE, Halley YA, et al. Genome-wide association study for feed efficiency and growth traits in U.S. beef cattle. BMC Genomics. 2017; 18:386.

52. Neibergs HL, Seabury CM, Wojtowicz AJ, Wang Z, Scraggs E, Kiser JN, et al. Susceptibility loci revealed for bovine respiratory disease complex in preweaned holstein calves. BMC Genomics. 2014;15:1164.

53. Bovine Genome Sequencing and Analysis Consortium, Elsik CG, Tellam RL, Worley KC, Gibbs RA, Muzny DM, et al. The genome sequence of taurine cattle: a window to ruminant biology and evolution. Science. 2009;324:522-8.

54. Bovine HapMap Consortium, Gibbs RA, Taylor JF, Van Tassell CP, Barendse W, Eversole KA, et al. Genome-wide survey of SNP variation uncovers the genetic structure of cattle breeds. Science. 2009;324:528-32.

55. Machugh DE, Loftus RT, Bradley DG, Sharp PM, Cunningham P. Microsatellite DNA variation within and among European cattle breeds. Proc Biol Sci. 1994;256:25-31.

56. MacHugh DE, Shriver MD, Loftus RT, Cunningham P, Bradley DG. Microsatellite DNA variation and the evolution, domestication and phylogeography of taurine and zebu cattle (Bos taurus and Bos indicus). Genetics. 1997;146:1071-86.

57. MacHugh DE, Loftus RT, Cunningham P, Bradley DG. Genetic structure of seven European cattle breeds assessed using 20 microsatellite markers. Anim Genet. 1998;29:333-40. 
58. Loftus RT, Ertugrul O, Harba AH, El-Barody MA, MacHugh DE, Park SD, et al. A microsatellite survey of cattle from a centre of origin: the Near East. Mol Ecol. 1999;8:2015-22.

59. Maudet C, Luikart G, Taberlet P. Genetic diversity and assignment tests among seven French cattle breeds based on microsatellite DNA analysis. J Anim Sci. 2002;80:942-50.

60. Heaton MP, Harhay GP, Bennett GL, Stone RT, Grosse WM, Casas E, et al. Selection and use of SNP markers for animal identification and paternity analysis in U.S. beef cattle. Mamm Genome. 2002;13:272-81.

61. Negrini R, Nicoloso L, Crepaldi P, Milanesi E, Colli L, Chegdani F, et al. Assessing SNP markers for assigning individuals to cattle populations. Anim Genet. 2009;40:18-26.

62. Matukumalli LK, Lawley CT, Schnabel RD, Taylor JF, Allan MF, Heaton MP, et al. Development and characterization of a high density SNP genotyping assay for cattle. PLoS One. 2009;4:e5350.

63. McKay SD, Schnabel RD, Murdoch BM, Matukumalli LK, Aerts J, Coppieters W, et al. An assessment of population structure in eight breeds of cattle using a whole genome SNP panel. BMC Genet. 2008;9:37.

64. Lewis J, Abas Z, Dadousis C, Lykidis D, Paschou P, Drineas P. Tracing cattle breeds with principal components analysis ancestry informative SNPs. PLoS One. 2011;6:e18007.

65. Raj A, Stephens M, Pritchard JK. fastSTRUCTURE: Variational Inference of Population Structure in Large SNP Data Sets. Genetics. 2014;197:573-89.

66. Wilkinson S, Wiener P, Archibald AL, Law A, Schnabel RD, McKay SD, et al. Evaluation of approaches for identifying population informative markers from high density SNP chips. BMC Genet. 2011;12:45.

67. Chen C-Y, Pollack S, Hunter DJ, Hirschhorn JN, Kraft P, Price AL. Improved ancestry inference using weights from external reference panels. Bioinformatics. 2013;29:1399-406.

68. Crum TE, Schnabel RD, Decker JE, Regitano LCA. CRUMBLER: A tool for the Prediction of Ancestry in Cattle. bioRxiv. 2018.

https://www.biorxiv.org/content/early/2018/08/20/396341.abstract.

69. Ramey HR, Decker JE, McKay SD, Rolf MM, Schnabel RD, Taylor JF. Detection of selective sweeps in cattle using genome-wide SNP data. BMC Genomics. 2013;14:382. 
70. Bhatia G, Tandon A, Patterson N, Aldrich MC, Ambrosone CB, Amos C, et al. Genome-wide scan of 29,141 African Americans finds no evidence of directional selection since admixture. Am J Hum Genet. 2014;95:437-44.

71. Kopp JB, Smith MW, Nelson GW, Johnson RC, Freedman BI, Bowden DW, et al. MYH9 is a major-effect risk gene for focal segmental glomerulosclerosis. Nat Genet. 2008;40:1175-84.

72. Finlay EK, Berry DP, Wickham B, Gormley EP, Bradley DG. A genome wide association scan of bovine tuberculosis susceptibility in Holstein-Friesian dairy cattle. PLoS One. 2012;7:e30545.

73. Bermingham ML, Bishop SC, Woolliams JA, Pong-Wong R, Allen AR, $\mathrm{McBride} \mathrm{SH}$, et al. Genome-wide association study identifies novel loci associated with resistance to bovine tuberculosis. Heredity . 2014;112:543-51.

74. Khayatzadeh N, Mészáros G, Utsunomiya YT, Garcia JF, Schnyder U, Gredler B, et al. Locus-specific ancestry to detect recent response to selection in admixed Swiss Fleckvieh cattle. Anim Genet. 2016;47:637-46.

75. Zhang Q, Calus MPL, Bosse M, Sahana G, Lund MS, Guldbrandtsen B. Human-Mediated Introgression of Haplotypes in a Modern Dairy Cattle Breed. Genetics. 2018;209:1305-17.

76. Lawson DJ, van Dorp L, Falush D. A tutorial on how not to over-interpret STRUCTURE and ADMIXTURE bar plots. Nat Commun. 2018;9:3258.

77. Purcell S, Neale B, Todd-Brown K, Thomas L, Ferreira MAR, Bender D, et al. PLINK: a tool set for whole-genome association and population-based linkage analyses. Am J Hum Genet. 2007;81:559-75.

78. Chang CC, Chow CC, Tellier LC, Vattikuti S, Purcell SM, Lee JJ. Secondgeneration PLINK: rising to the challenge of larger and richer datasets. Gigascience. 2015;4:7.

79. Patterson N, Price AL, Reich D. Population structure and eigenanalysis. PLoS Genet. 2006;2:e190.

80. Price AL, Patterson NJ, Plenge RM, Weinblatt ME, Shadick NA, Reich D. Principal components analysis corrects for stratification in genome-wide association studies. Nat Genet. 2006;38:904-9.

81. Wang J. The computer program structure for assigning individuals to populations: easy to use but easier to misuse. Mol Ecol Resour. 2017;17:981-90.

82. Puechmaille SJ. The program structure does not reliably recover the correct 
population structure when sampling is uneven: subsampling and new estimators alleviate the problem. Mol Ecol Resour. 2016;16:608-27.

83. Sanders JO. History and Development of Zebu Cattle in the United States. J Anim Sci. 1980;50:1188-200.

84. Kuehn LA, Keele JW, Bennett GL, McDaneld TG, Smith TPL, Snelling WM, et al. Predicting breed composition using breed frequencies of 50,000 markers from the US Meat Animal Research Center 2,000 Bull Project. J Anim Sci. 2011;89:1742-50.

85. Leesburg VLR, MacNeil MD, Neser FWC. Influence of Miles City Line 1 on the United States Hereford population. J Anim Sci. 2014;92:2387-94.

86. McCann LP. battle of bull runts. 1974. http://agris.fao.org/agrissearch/search.do?recordID=US201300539215.

87. Wiedemar N, Tetens J, Jagannathan V, Menoud A, Neuenschwander S, Bruggmann R, et al. Independent polled mutations leading to complex gene expression differences in cattle. PLoS One. 2014;9:e93435.

88. Whitacre L. Structural variation at the KIT locus is responsible for the piebald phenotype in Hereford and Simmental cattle. 2014.

http://search.proquest.com/openview/45eba5fa3c5757a2c4c2ab18af1a8a98/1?p q-origsite $=$ gscholar $\& \mathrm{cbl}=18750 \&$ diss $=\mathrm{y}$.

89. Chan EKF, Nagaraj SH, Reverter A. The evolution of tropical adaptation: comparing taurine and zebu cattle. Anim Genet. 2010;41:467-77.

90. Loftus RT, MacHugh DE, Bradley DG, Sharp PM, Cunningham P. Evidence for two independent domestications of cattle. Proc Natl Acad Sci U S A. 1994;91:2757-61.

91. Cartwright TC. Selection Criteria for Beef Cattle for the Future 1. J Anim Sci. 1970;30:706-11.

92. Dickerson G. Efficiency of Animal Production-Molding the Biological Components. J Anim Sci. 1970;30:849-59.

93. Porto-Neto LR, Reverter A, Prayaga KC, Chan EKF, Johnston DJ, Hawken RJ, et al. The genetic architecture of climatic adaptation of tropical cattle. PLoS One. 2014;9:e113284.

94. Goszczynski DE, Corbi-Botto CM, Durand HM, Rogberg-Muñoz A, Munilla S, Peral-Garcia $P$, et al. Evidence of positive selection towards Zebuine haplotypes in the BoLA region of Brangus cattle. Animal. 2018;12:215-23. 
95. Warwick EJ. Fifty years of progress in breeding beef cattle. J Anim Sci. 1958;17:922-43.

96. Thornton TA, Bermejo JL. Local and global ancestry inference and applications to genetic association analysis for admixed populations. Genet Epidemiol. 2014;38 Suppl 1:S5-12.

97. Tang $\mathrm{H}$, Peng J, Wang $\mathrm{P}$, Risch NJ. Estimation of individual admixture: analytical and study design considerations. Genet Epidemiol. 2005;28:289-301.

98. Rosen BD, Bickhart DM, Schnabel RD, Koren S, Elsik CG, Zimin A, et al. Modernizing the bovine reference genome assembly. In: Proceedings of the World Congress on Genetics Applied to Livestock Production (Auckland). 2018.

99. Loh P-R, Danecek P, Palamara PF, Fuchsberger C, A Reshef $Y$, K Finucane $\mathrm{H}$, et al. Reference-based phasing using the Haplotype Reference Consortium panel. Nat Genet. 2016;48:1443-8.

100. Das S, Forer L, Schönherr S, Sidore C, Locke AE, Kwong A, et al. Nextgeneration genotype imputation service and methods. Nat Genet. 2016;48:12847.

101. Rowan TN, Hoff JL, Crum TE, Taylor JF, Schnabel RD. A Multi-Breed Reference Panel and Additional Rare Variation Maximizes Imputation Accuracy in Cattle. bioRxiv. 2019.

https://www.biorxiv.org/content/10.1101/517144v1.abstract.

102. Maples BK, Gravel S, Kenny EE, Bustamante CD. RFMix: a discriminative modeling approach for rapid and robust local-ancestry inference. Am J Hum Genet. 2013;93:278-88.

103. Hu Z-L, Park CA, Reecy JM. Building a livestock genetic and genomic information knowledgebase through integrative developments of Animal QTLdb and CorrDB. Nucleic Acids Res. 2019;47:D701-10.

104. Rhoad AO. THE SANTA GERTRUDIS BREEDThe Genesis and the Genetics of a New Breed of Beef Cattle. J Hered. 1949;40:115-26.

105. Browning SR, Grinde K, Plantinga A, Gogarten SM, Stilp AM, Kaplan RC, et al. Local Ancestry Inference in a Large US-Based Hispanic/Latino Study: Hispanic Community Health Study/Study of Latinos (HCHS/SOL). G3 . 2016;6:1525-34.

106. Tenesa A, Navarro P, Hayes BJ, Duffy DL, Clarke GM, Goddard ME, et al. Recent human effective population size estimated from linkage disequilibrium. Genome Res. 2007;17:520-6. 
107. Frisch JE, Vercoe TE. An analysis of growth of different cattle genotypes reared in different environments. J Agric Sci. 1984;103:137-53.

108. Frisch JE, Vercoe JE. Food intake, eating rate, weight gains, metabolic rate and efficiency of feed utilization in Bos taurus and Bos indicus crossbred cattle. Anim Sci. 1977;25:343-58.

109. Menjo DK, Bebe BO, Okeyo AM, Ojango JMK. Analysis of early survival of Holstein-Friesian heifers of diverse sire origins on commercial dairy farms in Kenya. Trop Anim Health Prod. 2009;41:171-81.

110. Drögemüller $C$, Wöhlke $A$, Mömke $S$, Distl O. Fine mapping of the polled locus to a 1-Mb region on bovine chromosome 1q12. Mamm Genome. 2005;16:613-20.

111. Long CR, Gregory KE. Inheritance of the horned, scurred, and polled condition in cattle. J Hered. 1978;69:395-400.

112. Brenneman RA, Davis SK, Sanders JO, Burns BM, Wheeler TC, Turner JW, et al. The Polled Locus Maps to BTA1 in a Bos indicus $\times$ Bos taurus Cross. J Hered. 1996;87:156-61.

113. Whitacre L. Structural variation at the KIT locus is responsible for the piebald phenotype in Hereford and Simmental cattle. [University of Missouri-Columbia]; 2014. https://mospace.umsystem.edu/xmlui/handle/10355/44434. Accessed 25 Feb 2019.

114. Searle AG. Comparative genetics of coat colour in mammals. Comparative genetics of coat colour in mammals. 1968.

https://www.cabdirect.org/cabdirect/abstract/19680103237.

115. Giebel LB, Spritz RA. Mutation of the KIT (mast/stem cell growth factor receptor) protooncogene in human piebaldism. Proc Natl Acad Sci U S A. $1991 ; 88: 8696-9$.

116. Grosz MD, MacNeil MD, Others. The "Spotted" Locus Maps to Bovine Chromosome 6 in a HerefordCross Population. J Hered. 1999;90:233-5.

117. Liu L, Harris B, Keehan M, Zhang Y. Genome scan for the degree of white spotting in dairy cattle. Anim Genet. 2009;40:975-7.

118. Cooper MP, Fretwell N, Bailey SJ, Lyons LA. White spotting in the domestic cat (Felis catus) maps near KIT on feline chromosome B1. Anim Genet. 2006;37:163-5.

119. Brooks SA, Terry RB, Bailey E. A PCR-RFLP for KIT associated with 
tobiano spotting pattern in horses. Anim Genet. 2002;33:301-3.

120. Xu G-L, Ren J, Ding N-S, Ai H-S, Guo Y-M, Chen C-Y, et al. Genetic analysis of the KIT and MC1R genes in Chinese indigenous pigs with belt-like coat color phenotypes. Anim Genet. 2006;37:518-9.

121. Reinsch N, Thomsen H, Xu N, Brink M, Looft C, Kalm E, et al. A QTL for the degree of spotting in cattle shows synteny with the KIT locus on chromosome 6. J Hered. 1999;90:629-34.

122. Seitz JJ, Schmutz SM, Thue TD, Buchanan FC. A missense mutation in the bovine MGF gene is associated with the roan phenotype in Belgian Blue and Shorthorn cattle. Mamm Genome. 1999;10:710-2.

123. Mateescu RG, Garrick DJ, Reecy JM. Network Analysis Reveals Putative Genes Affecting Meat Quality in Angus Cattle. Front Genet. 2017;8:171.

124. Nalaila SM, Stothard P, Moore SS, Li C, Wang Z. Whole-genome QTL scan for ultrasound and carcass merit traits in beef cattle using Bayesian shrinkage method. J Anim Breed Genet. 2012;129:107-19.

125. Akanno EC, Chen L, Abo-Ismail MK, Crowley JJ, Wang Z, Li C, et al. Genome-wide association scan for heterotic quantitative trait loci in multi-breed and crossbred beef cattle. Genet Sel Evol. 2018;50:48.

126. Yeo J-S, Lee J-Y, Kim J-W. DNA marker mining of ILSTS035 microsatellite locus on chromosome 6 of Hanwoo cattle. J Genet. 2004;83:245-50.

127. Kneeland J, Li C, Basarab J, Snelling WM, Benkel B, Murdoch B, et al. Identification and fine mapping of quantitative trait loci for growth traits on bovine chromosomes 2, 6, 14, 19, 21, and 23 within one commercial line of Bos taurus. J Anim Sci. 2004;82:3405-14.

128. Nkrumah JD, Sherman EL, Li C, Marques E, Crews DH Jr, Bartusiak R, et al. Primary genome scan to identify putative quantitative trait loci for feedlot growth rate, feed intake, and feed efficiency of beef cattle. J Anim Sci. 2007;85:3170-81.

129. McClure MC, Morsci NS, Schnabel RD, Kim JW, Yao P, Rolf MM, et al. A genome scan for quantitative trait loci influencing carcass, post-natal growth and reproductive traits in commercial Angus cattle. Anim Genet. 2010;41:597-607.

130. Casas E, Keele JW, Shackelford SD, Koohmaraie M, Stone RT. Identification of quantitative trait loci for growth and carcass composition in cattle. Anim Genet. 2004;35:2-6. 
131. Howard JT, Kachman SD, Snelling WM, Pollak EJ, Ciobanu DC, Kuehn LA, et al. Beef cattle body temperature during climatic stress: a genome-wide association study. Int J Biometeorol. 2014;58:1665-72.

132. Michenet A, Barbat M, Saintilan R, Venot E, Phocas F. Detection of quantitative trait loci for maternal traits using high-density genotypes of Blonde d'Aquitaine beef cattle. BMC Genet. 2016;17:88.

133. Hawken RJ, Zhang YD, Fortes MRS, Collis E, Barris WC, Corbet NJ, et al. Genome-wide association studies of female reproduction in tropically adapted beef cattle. J Anim Sci. 2012;90:1398-410.

134. Meredith BK, Kearney FJ, Finlay EK, Bradley DG, Fahey AG, Berry DP, et al. Genome-wide associations for milk production and somatic cell score in Holstein-Friesian cattle in Ireland. BMC Genet. 2012;13:21.

135. Mömke S, Fink S, Wöhlke A, Drögemüller $C$, Distl O. Linkage of bilateral convergent strabismus with exophthalmus (BCSE) to BTA5 and BTA18 in German Brown cattle. Anim Genet. 2008;39:544-9.

136. Schulman NF, Sahana G, Lund MS, Viitala SM, Vilkki JH. Quantitative trait loci for fertility traits in Finnish Ayrshire cattle. Genet Sel Evol. 2008;40:195-214.

137. Neupane M, Kiser JN, Bovine Respiratory Disease Complex Coordinated Agricultural Project Research Team, Neibergs HL. Gene set enrichment analysis of SNP data in dairy and beef cattle with bovine respiratory disease. Anim Genet. 2018;49:527-38.

138. Psaros KM, McDaneld TG, Kuehn LA, Snelling WM, Keele JW. Evaluation of single nucleotide polymorphisms in chromosomal regions impacting pregnancy status in cattle. J Anim Sci. 2015;93:978-87.

139. Fortes MRS, Reverter A, Kelly M, McCulloch R, Lehnert SA. Genome-wide association study for inhibin, luteinizing hormone, insulin-like growth factor 1, testicular size and semen traits in bovine species. Andrology. 2013;1:644-50.

140. Doran AG, Berry DP, Creevey CJ. Whole genome association study identifies regions of the bovine genome and biological pathways involved in carcass trait performance in Holstein-Friesian cattle. BMC Genomics. 2014;15:837.

141. Ashwell MS, Heyen DW, Weller JI, Ron M, Sonstegard TS, Van Tassell CP, et al. Detection of quantitative trait loci influencing conformation traits and calving ease in Holstein-Friesian cattle. J Dairy Sci. 2005;88:4111-9.

142. Rodriguez-Zas SL, Southey BR, Heyen DW, Lewin HA. Detection of 
quantitative trait loci influencing dairy traits using a model for longitudinal data. $\mathrm{J}$ Dairy Sci. 2002;85:2681-91.

143. Olsen HG, Lien S, Svendsen M, Nilsen H, Roseth A, Aasland Opsal M, et al. Fine mapping of milk production QTL on BTA6 by combined linkage and linkage disequilibrium analysis. J Dairy Sci. 2004;87:690-8.

144. Cole JB, Wiggans GR, Ma L, Sonstegard TS, Lawlor TJ Jr, Crooker BA, et al. Genome-wide association analysis of thirty one production, health, reproduction and body conformation traits in contemporary U.S. Holstein cows. BMC Genomics. 2011;12:408.

145. Costa RB, Camargo GMF, Diaz IDPS, Irano N, Dias MM, Carvalheiro R, et al. Genome-wide association study of reproductive traits in Nellore heifers using Bayesian inference. Genet Sel Evol. 2015;47:67.

146. Frischknecht M, Bapst B, Seefried FR, Signer-Hasler H, Garrick D, Stricker $\mathrm{C}$, et al. Genome-wide association studies of fertility and calving traits in Brown Swiss cattle using imputed whole-genome sequences. BMC Genomics. 2017;18:910.

147. Cochran SD, Cole JB, Null DJ, Hansen PJ. Discovery of single nucleotide polymorphisms in candidate genes associated with fertility and production traits in Holstein cattle. BMC Genet. 2013;14:49.

148. Cochran SD, Cole JB, Null DJ, Hansen PJ. Single nucleotide polymorphisms in candidate genes associated with fertilizing ability of sperm and subsequent embryonic development in cattle. Biol Reprod. 2013;89:69.

149. Fang L, Sørensen P, Sahana G, Panitz F, Su G, Zhang S, et al. MicroRNAguided prioritization of genome-wide association signals reveals the importance of microRNA-target gene networks for complex traits in cattle. Sci Rep. 2018;8:9345.

150. Guo J, Jorjani H, Carlborg Ö. A genome-wide association study using international breeding-evaluation data identifies major loci affecting production traits and stature in the Brown Swiss cattle breed. BMC Genet. 2012;13:82.

151. Peñagaricano $F$, Weigel KA, Khatib $H$. Genome-wide association study identifies candidate markers for bull fertility in Holstein dairy cattle. Anim Genet. 2012;43 Suppl 1:65-71.

152. Cole JB, Waurich B, Wensch-Dorendorf M, Bickhart DM, Swalve HH. A genome-wide association study of calf birth weight in Holstein cattle using single nucleotide polymorphisms and phenotypes predicted from auxiliary traits. J Dairy Sci. 2014;97:3156-72. 
153. Ishii A, Yamaji K, Uemoto Y, Sasago N, Kobayashi E, Kobayashi N, et al. Genome-wide association study for fatty acid composition in Japanese Black cattle. Anim Sci J. 2013;84:675-82.

154. Ibeagha-Awemu EM, Peters SO, Akwanji KA, Imumorin IG, Zhao X. High density genome wide genotyping-by-sequencing and association identifies common and low frequency SNPs, and novel candidate genes influencing cow milk traits. Sci Rep. 2016;6:31109.

155. Iso-Touru T, Sahana G, Guldbrandtsen B, Lund MS, Vilkki J. Genome-wide association analysis of milk yield traits in Nordic Red Cattle using imputed whole genome sequence variants. BMC Genet. 2016;17:55.

156. Pszczola M, Strabel T, Mucha S, Sell-Kubiak E. Genome-wide association identifies methane production level relation to genetic control of digestive tract development in dairy cows. Sci Rep. 2018;8:15164.

157. McClure MC, Ramey HR, Rolf MM, McKay SD, Decker JE, Chapple RH, et al. Genome-wide association analysis for quantitative trait loci influencing Warner-Bratzler shear force in five taurine cattle breeds. Anim Genet. 2012;43:662-73.

158. Parker Gaddis KL, Null DJ, Cole JB. Explorations in genome-wide association studies and network analyses with dairy cattle fertility traits. J Dairy Sci. 2016;99:6420-35.

159. Chamberlain AJ, Hayes BJ, Savin K, Bolormaa S, McPartlan HC, Bowman $\mathrm{PJ}$, et al. Validation of single nucleotide polymorphisms associated with milk production traits in dairy cattle. J Dairy Sci. 2012;95:864-75.

160. Marete AG, Guldbrandtsen B, Lund MS, Fritz S, Sahana G, Boichard D. A Meta-Analysis Including Pre-selected Sequence Variants Associated With Seven Traits in Three French Dairy Cattle Populations. Front Genet. 2018;9:522.

161. Garcia MD, Matukumalli L, Wheeler TL, Shackelford SD, Smith TPL, Casas E. Markers on bovine chromosome 20 associated with carcass quality and composition traits and incidence of contracting infectious bovine keratoconjunctivitis. Anim Biotechnol. 2010;21:188-202.

162. Mömke S, Sickinger M, Lichtner P, Doll K, Rehage J, Distl O. Genome-wide association analysis identifies loci for left-sided displacement of the abomasum in German Holstein cattle. J Dairy Sci. 2013;96:3959-64.

163. Viitala S, Szyda J, Blott S, Schulman N, Lidauer M, Mäki-Tanila A, et al. The role of the bovine growth hormone receptor and prolactin receptor genes in milk, fat and protein production in Finnish Ayrshire dairy cattle. Genetics. 
2006;173:2151-64.

164. Arranz JJ, Coppieters W, Berzi P, Cambisano N, Grisart B, Karim L, et al. A QTL affecting milk yield and composition maps to bovine chromosome 20: a confirmation. Anim Genet. 1998;29:107-15.

165. Wang X, Ma P, Liu J, Zhang Q, Zhang Y, Ding X, et al. Genome-wide association study in Chinese Holstein cows reveal two candidate genes for somatic cell score as an indicator for mastitis susceptibility. BMC Genet. 2015;16:111.

166. Peters SO, Kizilkaya K, Garrick DJ, Fernando RL, Reecy JM, Weaber RL, et al. Bayesian genome-wide association analysis of growth and yearling ultrasound measures of carcass traits in Brangus heifers. J Anim Sci. 2012;90:3398-409.

167. Saatchi M, Schnabel RD, Taylor JF, Garrick DJ. Large-effect pleiotropic or closely linked QTL segregate within and across ten US cattle breeds. BMC Genomics. 2014;15:442.

168. Buitenhuis B, Janss LLG, Poulsen NA, Larsen LB, Larsen MK, Sørensen P. Genome-wide association and biological pathway analysis for milk-fat composition in Danish Holstein and Danish Jersey cattle. BMC Genomics. 2014;15:1112.

169. Dickerson GE, Künzi N, Cundiff LV, Koch RM, Arthaud VH, Gregory KE. Selection Criteria for Efficient Beef Production. J Anim Sci. 1974;39:659-73.

170. Koch RM, Swiger LA, Chambers D, Gregory KE. Efficiency of Feed Use in Beef Cattle. J Anim Sci. 1963;22:486-94.

171. Lowe M, Gereffi G. A value chain analysis of the US beef and dairy industries. Center on Globalization, Governance \& Competitiveness, Duke University. 2009.

https://www.researchgate.net/profile/Marcy_Lowe2/publication/294579508_A_Va lue_Chain_Analysis_of_the_US_Beef_and_Dairy_Industries/links/56c1e25308ae e5caccf99b1f.pdf.

172. Archer JA, Richardson EC, Herd RM, Arthur PF. Potential for selection to improve efficiency of feed use in beef cattle: a review. Aust J Agric Res. 1999;50:147-62.

173. Deutscher GH. Reducing calving difficulty by heifer and sire selection and management. In: Range Beef Cow Symposium. digitalcommons.unl.edu; 1995. p. 183.

174. Kennedy BW, van der Werf JH, Meuwissen TH. Genetic and statistical 
properties of residual feed intake. J Anim Sci. 1993;71:3239-50.

175. Lu D, Miller S, Sargolzaei M, Kelly M, Vander Voort G, Caldwell T, et al. Genome-wide association analyses for growth and feed efficiency traits in beef cattle. J Anim Sci. 2013;91:3612-33.

176. Serão NV, González-Peña D, Beever JE, Faulkner DB, Southey BR, Rodriguez-Zas SL. Single nucleotide polymorphisms and haplotypes associated with feed efficiency in beef cattle. BMC Genet. 2013;14:94.

177. Crowley JJ, McGee M, Kenny DA, Crews DH Jr, Evans RD, Berry DP. Phenotypic and genetic parameters for different measures of feed efficiency in different breeds of Irish performance-tested beef bulls. J Anim Sci. 2010;88:88594.

178. Liu MF, Goonewardene LA, Bailey DRC, Basarab JA, Kemp RA, Arthur PF, et al. A study on the variation of feed efficiency in station tested beef bulls. Can $\mathrm{J}$ Anim Sci. 2000;80:435-41.

179. Snelling WM, Allan MF, Keele JW, Kuehn LA, McDaneld T, Smith TPL, et al. Genome-wide association study of growth in crossbred beef cattle. J Anim Sci. 2010;88:837-48.

180. Bolormaa S, Hayes BJ, Savin K, Hawken R, Barendse W, Arthur PF, et al. Genome-wide association studies for feedlot and growth traits in cattle. J Anim Sci. 2011;89:1684-97.

181. Barendse W, Reverter A, Bunch RJ, Harrison BE, Barris W, Thomas MB. A validated whole-genome association study of efficient food conversion in cattle. Genetics. 2007;176:1893-905.

182. Sherman EL, Nkrumah JD, Moore SS. Whole genome single nucleotide polymorphism associations with feed intake and feed efficiency in beef cattle. $J$ Anim Sci. 2010;88:16-22.

183. Zhou X, Stephens M. Genome-wide efficient mixed-model analysis for association studies. Nat Genet. 2012;44:821-4.

184. Kang HM, Sul JH, Service SK, Zaitlen NA, Kong S-Y, Freimer NB, et al. Variance component model to account for sample structure in genome-wide association studies. Nat Genet. 2010;42:348-54.

185. Segura V, Vilhjálmsson BJ, Platt A, Korte A, Seren Ü, Long Q, et al. An efficient multi-locus mixed-model approach for genome-wide association studies in structured populations. Nat Genet. 2012;44:825-30. 
186. Zhou X, Carbonetto P, Stephens M. Polygenic modeling with bayesian sparse linear mixed models. PLoS Genet. 2013;9:e1003264.

187. McRae AF, McEwan JC, Dodds KG, Wilson T, Crawford AM, Slate J. Linkage disequilibrium in domestic sheep. Genetics. 2002;160:1113-22.

188. Onteru SK, Fan B, Du Z-Q, Garrick DJ, Stalder KJ, Rothschild MF. A wholegenome association study for pig reproductive traits. Anim Genet. 2012;43:1826.

189. Mittapalli VR, Pröls F, Huang R, Christ B, Scaal M. Avian stanniocalcin-2 is expressed in developing striated muscle and joints. Anat Embryol . 2006;211:519-23.

190. Gagliardi AD, Kuo EYW, Raulic S, Wagner GF, DiMattia GE. Human stanniocalcin-2 exhibits potent growth-suppressive properties in transgenic mice independently of growth hormone and IGFs. Am J Physiol Endocrinol Metab. 2005;288:E92-105.

191. Sharma NK, Das SK, Mondal AK, Hackney OG, Chu WS, Kern PA, et al. Endoplasmic reticulum stress markers are associated with obesity in nondiabetic subjects. J Clin Endocrinol Metab. 2008;93:4532-41.

192. Linnemann A, van der Ven PFM, Vakeel P, Albinus B, Simonis D, Bendas $G$, et al. The sarcomeric Z-disc component myopodin is a multiadapter protein that interacts with filamin and $\alpha$-actinin. Eur J Cell Biol. 2010;89:681-92.

193. Utsunomiya YT, do Carmo AS, Carvalheiro R, Neves HHR, Matos MC, Zavarez LB, et al. Genome-wide association study for birth weight in Nellore cattle points to previously described orthologous genes affecting human and bovine height. BMC Genet. 2013;14:52.

194. Gudbjartsson DF, Walters GB, Thorleifsson G, Stefansson H, Halldorsson $\mathrm{BV}$, Zusmanovich $\mathrm{P}$, et al. Many sequence variants affecting diversity of adult human height. Nat Genet. 2008;40:609-15.

195. Pryce JE, Hayes BJ, Bolormaa S, Goddard ME. Polymorphic Regions Affecting Human Height Also Control Stature in Cattle. Genetics. 2011;187:9814. doi:10.1534/genetics.110.123943.

196. Nishimura S, Watanabe T, Mizoshita K, Tatsuda K, Fujita T, Watanabe N, et al. Genome-wide association study identified three major QTL for carcass weight including the PLAG1-CHCHD7 QTN for stature in Japanese Black cattle. BMC Genet. 2012;13:40.

197. Porto Neto LR, Bunch RJ, Harrison BE, Barendse W. Variation in the XKR4 
gene was significantly associated with subcutaneous rump fat thickness in indicine and composite cattle. Anim Genet. 2012;43:785-9.

198. Patwari P, Emilsson V, Schadt EE, Chutkow WA, Lee S, Marsili A, et al. The arrestin domain-containing 3 protein regulates body mass and energy expenditure. Cell Metab. 2011;14:671-83.

199. Mersmann HJ. Overview of the effects of beta-adrenergic receptor agonists on animal growth including mechanisms of action. J Anim Sci. 1998;76:160-72.

200. Beermann DH. Beta-Adrenergic receptor agonist modulation of skeletal muscle growth. J Anim Sci. 2002;80:E18-23.

201. Zimin AV, Delcher AL, Florea L, Kelley DR, Schatz MC, Puiu D, et al. A whole-genome assembly of the domestic cow, Bos taurus. Genome Biol. 2009;10:R42.

202. Yang J, Weedon MN, Purcell S, Lettre G, Estrada K, Willer CJ, et al. Genomic inflation factors under polygenic inheritance. Eur J Hum Genet. 2011;19:807-12. 


\section{VITA}

Tamar Ellen (Adcock) Crum was born on October 10, 1991 in Assumption, Illinois. Throughout her youth, Tamar exhibited cattle across the nation with her family and gained professional and leadership experience through terms served with both national and state junior beef breed associations. Her family's seedstock program and her successful show cattle career sparked an interest in animal breeding and genetics. After graduating from Central A\&M High School in 2010, Tamar attended Kansas State University to pursue a degree in Animal Science with a minor in Agricultural Economics. Throughout her time at Kansas State University, Tamar became involved in an Honors research project with Animal Breeder/Geneticist and her undergraduate advisor, Dr. Dan Moser. This project sparked an interest in research within the field of genetics.

Tamar began a Doctoral program at the University of Missouri with Dr. Jerry Taylor in 2014. Through her graduate training, she has learned the importance of developing repeatable and systematic approaches to solving problems, to efficiently and effectively test and error correct routine processes, and to manage large amounts of data. Beyond just being able to conduct and manage research, throughout her undergraduate and graduate careers, Tamar has had numerous speaking and writing opportunities that have developed her ability to effectively communicate complex materials to a wide range of audiences. Tamar looks forward to continuing contributing to genetic research with The Maschhoffs in Carlyle, IL following graduation. 